\title{
Evolution of Bicoid-dependent hunchback Regulation in Diptera
}

\section{PhD Thesis}

\author{
in partial fulfillment of the requirements \\ for the degree "Doctor of Philosophy (PhD)" \\ in the Molecular Biology Program \\ at the Georg August University Göttingen, \\ Faculty of Biology
}

submitted by

Steffen Joachim Lemke

born in

Wuppertal 
Herewith I declare that I prepared the PhD Thesis "Evolution of Bicoid-dependent hunchback Regulation in Diptera" on my own and with no other sources and aids than quoted.

Chicago, 2006 
Parts of this work were published in the following meeting presentations :

Lemke SJ, Rafiqi AM, Prell AH, Stauber M, Schmidt-Ott U. Evolution of transcriptional control of the Drosophila gap gene hunchback. 45th Annual Drosophila Research Conference, March 24-28, 2004. Washington, DC USA. Poster.

Lemke SJ, Prell AH, Stauber M, Schmidt-Ott U. Evolution of transcriptional control of the Drosophila gap gene hunchback. 44th Annual Drosophila Research Conference, March 5-9, 2003. Chicago, Illinois USA. Poster. 


\section{Table of Contents}

$\begin{array}{ll}\text { Abstract } & 1\end{array}$

1 Introduction 2

1.1 Comparative embryology of Diptera 2

1.2 Pattern formation in Drosophila 4

$\begin{array}{lll}1.3 & \text { How did hunchback regulation in dipterans evolve? } & 7\end{array}$

1.3.1 hunchback in Drosophila 7

$\begin{array}{ll}\text { 1.3.2 hunchback regulation in Drosophila } & 7\end{array}$

1.3.3 hunchback regulation in dipterans and other insects 9

1.3.4 Complementary approaches to explore the evolution of hunchback regulation 12

$2 \quad$ Material and Methods $\quad 14$

$\begin{array}{lll}2.1 & \text { Fly culture and egg collection } & 14\end{array}$

$\begin{array}{lll}2.2 & \text { Cloning } & 15\end{array}$

$\begin{array}{ll}\text { 2.2.1 Preparation of genomic phage libraries } & 15\end{array}$

$\begin{array}{ll}2.2 .2 & \text { Preparation of cDNA templates } \\ 2.3 & 15\end{array}$

$\begin{array}{lll}2.2 .3 & \text { Isolation of homeobox genes } & 16\end{array}$

$\begin{array}{lll}2.2 .4 & \text { Isolation of hunchback homologues } & 17\end{array}$

$\begin{array}{lll}\text { 2.2.5 Isolation of hunchback genomic DNA } & 17\end{array}$

2.3 In situ hybridization, immunocytochemistry and microscopy 21

2.3.1 Embryo fixation 21

$\begin{array}{lll}2.3 .2 & \text { RNA in situ probes } & 21\end{array}$

2.3.3 Whole mount RNA in situ hybridization 21

2.4 cDNA library from embryonic pole cytoplasm 22

2.4.1 cDNA preparation from pole cytoplasm 22

2.4.2 Preparation of bacterial libraries from amplified cDNA 23

2.4.3 Hybridization of the libraries and subtractive screening 23

$\begin{array}{lll}2.4 .4 & \text { Virtual northern hybridization } & 24\end{array}$ 
$3 \quad$ Results $\quad 24$

3.1 Identification of bicoid orthologues from Platypeza and Lonchoptera 24

3.2 Subtractive screening for bicoid-like genes: a new method 25

3.3 Subtractive screening for bicoid-like genes in Clogmia 27

3.4 Cloning of dipteran hunchback genes 29

3.5 Functional comparison of early dipteran hunchback regulation 31

3.6 Expression studies in Episyrphus 33

3.6.1 Episyrphus hunchback shares expression characteristics of cyclorrhaphan and non-cyclorrhaphan dipterans 33

3.6.2 The extraembryonic anlage of Episyrphus extends to the anterior pole 34

3.6.3 Episyrphus orthodenticle is not expressed in pre-blastoderm embryos 36

$4 \quad$ Discussion $\quad 37$

4.1 Do all cyclorrhaphan dipterans have bicoid? 37

4.2 Does Episyrphus reflect the primitive patterning mechanism of cyclorrhaphan flies? 38

4.3 How did early anterior hunchback regulation change in dipteran evolution? 39

$\begin{array}{ll}\text { Summary and Conclusions } & \mathbf{4 0}\end{array}$

$\begin{array}{lll}\text { A } & \text { Appendix } & 41\end{array}$

$\begin{array}{lll}\text { A.1 } & \text { Figures and Tables } & 41\end{array}$

A.1.1 Figures $\quad 41$

$\begin{array}{lll}\text { A.1.2 Tables } & 59\end{array}$

$\begin{array}{llr}\text { A.2 } & \text { Supplemental Figures } & 64\end{array}$

$\begin{array}{lll}\text { A.3 Sequences } & 66\end{array}$

$\begin{array}{ll}\text { Bibliography } & 79\end{array}$ 


\section{List of Figures}

Figure 1. Phylogenetic relationships of taxa mentioned in the text.

Figure 2. Homeodomain alignment and percent sequence similarity relative to the homeodomains of Platypeza Bicoid and Lonchoptera Bicoid.

Figure 3. Protein alignment of bicoid homologues.

Figure 4. cDNA pools from anterior and posterior cytoplasm of the same Megaselia embryo differ in composition.

Figure 5. Identification of Megaselia bicoid by subtractive screening of a spotted cDNA library made from anterior egg cytoplasm.

Figure 6. Classification and abundance of Clogmia clones.

Figure 7. Testing for differential expression of Clogmia candidates in virtual Northern Blots.

Figure 8. Quartet Puzzling analysis of the newly identified Hunchback homologues.

Figure 9. Alignment of predicted protein sequences of the newly identified hunchback homologues.

Figure 10. Genomic organization of Dipteran hunchback homologues.

Figure 11. Putative nanos response element (NRE) sequences from dipteran hunchback homologues.

Figure 12. Functional analysis of hunchback regulatory DNA by reporter gene expression in Drosophila embryos.

Figure 13. Effects of bicoid RNAi on hunchback expression in Megaselia.

Figure 14. Expression of Episyrphus hunchback.

Figure 15. In Episyrphus, hunchback and zerknüllt are co-expressed along the dorsal midline.

Figure 16. Expression of Episyrphus zerknüllt.

Figure 17. Protein alignment of Episyrphus Orthodenticle with two isoforms of Drosophila Orthodenticle/Ocelliless.

Figure 18. Expression of Episyrphus orthodenticle. 


\section{List of Tables}

Table 1. cDNA isolation of dipteran bicoid and orthodenticle homologues: templates, primers and products.

Table 2. cDNA isolation of dipteran hunchback homologues: templates, primers and products.

Table 3. RNA probes for whole mount in situ hybridization.

Table 4. Sequence comparison of Clogmia candidates with Drosophila and Anopheles genes.

Table 5. Reporter gene constructs to analyze hunchback regulatory DNA in transgenic Drosophila embryos. 


\section{Acknowledgements}

The presented thesis has been carried out at the Max-Planck-Institut für biophysikalische Chemie in Göttingen, in the Department Molekulare Entwicklungsbiologie, and at the University of Chicago, in the Department of Organismal Biology and Anatomy. First of all I would like to thank my advisor, Urs Schmidt-Ott for his years of patient teaching and supervision, for his help, advice, and for his encouragement to follow up my own ideas. I would like to thank my committee members Herbert Jäckle and Reinhard Jahn for their critical interest and support. I thank Ab. Matteen Rafiqi, Sean Ferguson, Yu-Chiun Wang, Olivia Casanueva and Chip Ferguson, and of the former Göttinger group especially Michael Stauber, Alexander Prell and Ralf Pflanz for help and discussions. A special thanks goes to my parents for support and trust in me, and Annette, for her affection and patience.

\section{Contributions}

cDNA: Platypeza bicoid was cloned by Ab. Matteen Rafiqi (MR). Lonchoptera tristis bicoid, Episyrphus orthodenticle, and a zygotic transcript of Empis-hunchback were cloned by Michael Stauber (MS). A full-length cDNA of Clogmia hunchback and a zinc finger fragment of Haematopota hunchback were cloned by Alexander Prell (AP). Genomic DNA: The Megaselia hunchback locus was cloned by MS. The Clogmia hunchback locus was cloned by AP. Reporter constructs: Transgenic Drosophila lines carrying the Megaselia hunchback locus were established by MS. Transgenic Drosophila lines carrying the reporter constructs with Clogmia hunchback regulatory DNA were established and analyzed by AP. Fly work: Technical assistance with RNAi in Megaselia, removal of cytoplasm, and fixation of embryos was provided by Sean Ferguson (SF). cDNA library from anterior cytoplasm: The library was established as a glycerol stock in collaboration with MR; MR, SF, Urs Schmidt-Ott, and Irene Hsiao helped with colony picking. The library was spotted by MR in collaboration with Professor Helmut Bloecker at the Department of Genome Analysis, German Research Center for Biotechnology, Braunschweig, Germany 


\section{Abstract}

An early segmentation gene of Drosophila melanogaster, hunchback, with an evolutionarily conserved function but diverging regulation was used as an entry point to explore the evolution of early patterning mechanisms in true flies (Diptera). In Drosophila, a gradient of bicoid protein activates the transcription of hunchback in the anterior blastoderm and thereby initiates patterning of the thorax. Very similar hunchback expression has been reported for other dipterans but a correlation with the occurrence of bicoid could not be established. Therefore, one or several hunchback regulators may have been exchanged in dipteran evolution. To map this transition in the regulation of hunchback expression, I expanded previous screens for bicoid orthologues using low stringency PCR and cDNA subtraction as technical approaches, and compared the results to the response of hunchback promoters from the same species using reporter constructs in transgenic Drosophila. Reporter expression in the anterior blastoderm of transgenic Drosophila was recorded only when the promoter was taken from a species with a bicoid orthologue. The reporter constructs of the hunchback promoters of all other species (five out of eight) were expressed in the posterior (2) or extraembryonic blastoderm (1), or were not expressed at all (2). These experiments

enabled me to identify a lower cyclorrhaphan fly (Episyrphus balteatus; Syrphidae) with an early patterning mechanism likely to be fundamentally different from Drosophila and potentially similar to lower dipterans. To explore the possibility that Episyrphus shares developmental traits with lower dipterans, I studied the expression of Episyrphus hunchback, Episyrphus zerknüllt, and Episyrphus orthodenticle and compared the expression of these genes to their direct homologues in Megaselia abdita, Drosophila and Clogmia albipunctata. I found that Episyrphus combines expression characteristics of cyclorrhaphan and non-cyclorrhaphan dipterans indicating that this species might use a patterning mechanism that is an intermediate between lower and higher flies. 


\section{Introduction}

The genetic basis of morphological evolution has received much attention in recent years (reviewed by Orr, 2005). Yet many genetic interactions change in the course of evolution without affecting morphology in an obvious way (reviewed e.g. by Raff, 1996). These changes might reflect neutral evolution of the developmental gene network (Raff, 1996), or alternatively they could be an adaptation to the developmental process itself (e.g. Bullock et al., 2004). In flies (Diptera), the segmentation gene hunchback provides a striking example for this phenomenon: early zygotic expression of this gene is very similar across dipterans, while the regulation of hunchback expression has undergone fundamental changes. To understand the evolutionary significance of this transition, I have explored the evolution of hunchback regulation in its phylogenetic context.

\subsection{Comparative embryology of Diptera}

In Dipterans, as in most other insects, the zygote nucleus divides without cell division (reviewed in Anderson, 1966; Anderson, 1972). After a series of four to ten nuclear divisions, most nuclei migrate to the periphery and form a monolayer around the yolk (Anderson, 1966). This layer of nuclei is referred to as syncytial blastoderm (Anderson, 1966). When the plasmamembrane folds inwards between the nuclei, the syncytial blastoderm turns into a cellular blastoderm (Anderson, 1966), although in Drosophila melanogaster ${ }^{1}$ at least, the cells do not pinch off completely from the underlying yolky cytoplasm until early gastrulation (Foe and Alberts, 1983).

By the onset of gastrulation, most of the dipteran blastoderm has been specified to become embryonic tissue, which is also referred to as germband (Johannsen and Butt, 1941); the remaining portion of the blastoderm will give rise to extraembryonic cell layers (Anderson, 1972; Johannsen and Butt, 1941). Shortly after the onset of gastrulation, the germband begins to extend from the posterior pole to the dorsal and then anteriorly such that the cells destined to form the most posterior larval structures are located transiently directly behind the future head region (Anderson, 1966; Campos-Ortega and Hartenstein, 1997). During germband re-

\footnotetext{
${ }^{1}$ Referred to as Drosophila in the remaining text. In the same way I will refer to other species by only their genus name after the first introduction in the main text.
} 
traction, this process is reversed (Anderson, 1966; Campos-Ortega and Hartenstein, 1997). In the retracting germband, segmental grooves form, which demarcate, in anterior-posterior sequence, the head, thoracic, and abdominal segments of the embryo (Anderson, 1966; CamposOrtega and Hartenstein, 1997). After germband retraction, the epidermis closes dorsally. At this stage, all organs are established and the epidermis of the embryo secretes the exoskeleton (cuticle) of the larva (Anderson, 1966; Campos-Ortega and Hartenstein, 1997).

This developmental blueprint varies in some aspects among dipterans. Most higher dipterans (Cyclorrhapha, Figure 1) and also culicomorphan mosquitoes (Culicomorpha, Figure 1) such as Anopheles gambiae develop according to an extreme long-germ mode of insect development (Anderson, 1972; Sander, 1976). In these taxa, all segments are specified prior to gastrulation (Bullock et al., 2004; Goltsev et al., 2004b), the germ band extends to the anterior pole, and the extraembryonic tissue originates from dorsal blastoderm only (Anderson, 1972; Sander, 1976). By contrast, some (probably most) lower dipterans retain a more ancestral mode of development. Similar to the intermediate or short-germ development of most holometabolous insects, the posterior-most segments of these lower dipterans are specified in a posterior "growth zone" after the onset of gastrulation, and the extraembryonic anlage extends to the anterior pole (Anderson, 1972; Sander, 1976). Apart from the size of the extraembryonic anlage, cyclorrhaphan and non-cyclorrhaphan dipterans also differ in the organization of extraembryonic tissue. The extraembryonic tissue of non-cyclorrhaphan dipterans, as in most holometabolous insects, differentiates into two cell layers, the amnion and the serosa (Anderson, 1966; Anderson, 1972; Schmidt-Ott, 2000). The amnion remains linked to the embryo and covers the ventral side of the embryo after germband retraction, whereas the serosa detaches from the embryonic tissue to completely close around the embryo and the yolk (Handel et al., 2000; Schwalm, 1987). By contrast, the extraembryonic tissue of higher cyclorrhaphans (Schizophora, Figure 1) is a derived character and consists of only a single cell layer that covers the yolk sac dorsally, the amnioserosa (Anderson, 1966; Anderson, 1972). 


\subsection{Pattern formation in Drosophila}

The molecular basis of dipteran segmentation has been studied primarily in Drosophila, where many segmentation genes have been discovered through saturating genetic screens for female sterile or embryonic lethal mutations, many of which cause phenotypes in the larval cuticle (Gans et al., 1975; Jürgens et al., 1984; Mohler, 1977; Nüsslein-Volhard et al., 1987; Nüsslein-Volhard and Wieschaus, 1980; Nüsslein-Volhard et al., 1984; Perrimon et al., 1986; Schüpbach and Wieschaus, 1989; Wieschaus et al., 1984). Depending on the cuticle phenotypes, the maternal genes were classified into four distinct maternal systems of anterior, posterior, terminal, and dorsal-ventral genes (Nüsslein-Volhard et al., 1987; St Johnston and Nüsslein-Volhard, 1992). The zygotic genes were classified according to their mutant phenotypes in the cuticle as gap genes, pair-rule genes, and segment polarity genes (NüssleinVolhard and Wieschaus, 1980). Loss-of-function mutations in these zygotic genes cause either missing blocks of segments in the cuticle (gap genes), defects in every other segment (pairrule genes), or an altered polarity of each segment (segment polarity genes) (Nüsslein-Volhard and Wieschaus, 1980). The Hox genes are involved in giving the embryonic segments their individual identity and were discovered independently (Lewis, 1978).

The anterior-posterior body axis is established by the anterior, the posterior, and the terminal maternal system (Nüsslein-Volhard et al., 1987). The anterior system is required for head and thorax development (Nüsslein-Volhard et al., 1987). The key gene is bicoid $^{2}$ (Berleth et al., 1988; Frohnhöfer and Nüsslein-Volhard, 1986). bicoid transcripts become enriched at the anterior pole of the oocyte during oogenesis (Berleth et al., 1988; Cha et al., 2001). Translation of the localized bicoid transcripts and assumed diffusion of the protein establish a Bicoid gradient along the anterior-posterior axis of the embryo (Driever and Nüsslein-Volhard, 1988). Bicoid binds to the ubiquitous transcript of caudal (Macdonald and Struhl, 1986; Mlodzik et al., 1985; Mlodzik and Gehring, 1987a; Rivera-Pomar et al., 1996) and represses its translation (Rivera-Pomar et al., 1996). Thus, Bicoid induces a Caudal gradient complementary to the Bicoid gradient (Macdonald and Struhl, 1986; Mlodzik and Gehring, 1987a; Mlodzik and Gehring, 1987b; Rivera-Pomar et al., 1996). In addition, Bicoid binds DNA (Driever and Nüsslein-Volhard, 1989; Struhl et al., 1989) and directly activates the tran-

\footnotetext{
${ }^{2}$ Nomenclature of genes and gene products is according to Drysdale et al. (2005).
} 
scription of the gap gene hunchback (Driever and Nüsslein-Volhard, 1989; Driever et al., 1989; Lehmann and Nüsslein-Volhard, 1987a; Struhl et al., 1989; Tautz et al., 1987) as well as a number of other gap and pair-rule genes (reviewed in Pankratz and Jäckle, 1993; reviewed in Rivera-Pomar and Jäckle, 1996).

The posterior system controls the establishment of a germ line and abdominal segmentation (Nüsslein-Volhard et al., 1987). The key genes are oskar (Ephrussi et al., 1991; KimHa et al., 1991; Lehmann and Nüsslein-Volhard, 1986) and nanos (Lehmann and NüssleinVolhard, 1991; Nüsslein-Volhard et al., 1987; Wang and Lehmann, 1991). Oskar recruits factors for the germ line, including the nanos transcript, to the posterior pole (Ephrussi et al., 1991; Ephrussi and Lehmann, 1992). Translation of the localized nanos transcript generates, possibly by diffusion, a Nanos gradient opposite to the Bicoid gradient (Gavis and Lehmann, 1992). Nanos is essential for abdominal segmentation as it suppresses the translation of maternal hunchback transcript in the posterior half of the early embryo (Hülskamp et al., 1989; Irish et al., 1989; Struhl, 1989; Tautz, 1988).

The terminal maternal system is required for the formation of the terminal body parts (Nüsslein-Volhard et al., 1987). The key gene is torso (Casanova and Struhl, 1989; Schüpbach and Wieschaus, 1986; Sprenger et al., 1989), which codes for a receptor tyrosine kinase (Sprenger et al., 1989; Sprenger et al., 1993). Torso is expressed evenly on the surface of the blastoderm embryo (Casanova and Struhl, 1989), but the receptor tyrosine kinase signaling pathway is activated only at the anterior and the posterior pole (Gabay et al., 1997). At both poles, the activated signaling pathway leads to the de-repression of tailless (Jiménez et al., 2000; Paroush et al., 1997; Pignoni et al., 1990), which is required for setting up the terminal structures of the larval cuticle (Jürgens et al., 1984).

The dorsal-ventral body axis of the embryo is established independently from the anterior-posterior body axis (St Johnston and Nüsslein-Volhard, 1992). The key gene is dorsal (Nüsslein-Volhard, 1979; Steward, 1987; Steward et al., 1984), which codes for a transcription factor (Thisse et al., 1991). Dorsal is ubiquitously distributed in the cytoplasm of the freshly laid egg (Roth et al., 1989; Rushlow et al., 1989; Steward, 1989). In response to an extracellular signaling cascade (reviewed in Moussian and Roth, 2005), Dorsal enters the nuclei on the prospective ventral side of the embryo (Roth et al., 1989; Rushlow et al., 1989; 
Steward, 1989). Due to its asymmetry, the signaling cascade creates a nuclear Dorsal gradient in the ventral half of the embryo, with highest levels of Dorsal in the ventral-most nuclei (Moussian and Roth, 2005; Roth et al., 1989; Rushlow et al., 1989; Steward, 1989). This Dorsal gradient subdivides the embryo axis into three main regions - ventral (presumptive mesoderm), lateral (presumptive neuroectoderm) and dorsal (presumptive ectoderm) - by triggering threshold responses from a number of zygotic patterning genes (reviewed by Stathopoulos and Levine, 2002). In the dorsal half of the blastoderm embryo, decapentaplegic (dpp; Padgett $e t$ al., 1987; Spencer et al., 1982) is expressed due to the absence of nuclear Dorsal (Ray et al., 1991). $d p p$ encodes a transforming growth factor- $\beta$ (TGF- $\beta$ ) protein (Padgett et al., 1987), which, together with the TGF- $\beta$ protein Screw (Arora et al., 1994; Nüsslein-Volhard et al., 1984), establishes a signaling center along the dorsal midline (reviewed in Ashe, 2005; reviewed in Raftery and Sutherland, 2003). Screw and peak levels of Dpp along the dorsal midline are required to specify the extraembryonic anlage (Arora et al., 1994; Ferguson and Anderson, 1992), which is established by Dpp dependent activation of zerknüllt (Doyle et al., 1986; Rushlow et al., 2001; Wakimoto et al., 1984).

Deviations from the Drosophila paradigm of early pattern formation have been reported for several non-cyclorrhaphan dipterans (Bullock et al., 2004; Goltsev et al., 2004b; Rohr et al., 1999; Stauber et al., 1999; Stauber et al., 2002). In Coboldia fuscipes and Clogmia albipunctata (Figure 1), for example, the onset of posterior pair-rule gene expression is delayed (Rohr et al., 1999), which correlates with the observation of a posterior "growth zone" in these species (Anderson, 1972). Furthermore, unlike in Drosophila, hunchback in noncyclorrhaphan dipterans is expressed in the presumptive extraembryonic anlage (Goltsev et al., 2004a; Rohr et al., 1999). This expression indicates a potential role of hunchback in extraembryonic development that could relate morphological differences in extraembryonic development between cyclorrhaphan and non-cyclorrhaphan dipterans. Most intriguingly, however, bicoid is absent from the Anopheles genome (Zdobnov et al., 2002), and, in addition to other Drosophila species (Drysdale et al., 2005), bicoid homologues have been found only in cyclorrhaphan flies (Schröder and Sander, 1993; Sommer and Tautz, 1991; Stauber et al., 1999). These and other studies led to the hypothesis that bicoid evolved only recently and is confined to cyclorrhaphan flies (Schmidt-Ott, 2000; Stauber et al., 2002). Sequence data sug- 
gest that bicoid and zerknüllt are sister genes, which most likely emerged from a Hox3 gene duplication in the stem lineage of Cyclorrhapha (Stauber et al., 1999; Stauber et al., 2002). This postulation is supported by the finding that the closest homologue of bicoid in the Anopheles genome is zerknüllt (own observation). Thus, the anterior patterning mechanism of flies must have changed with the emergence of bicoid. In particular, the regulation of its Drosophila target gene hunchback must have changed accordingly.

\subsection{How did hunchback regulation in dipterans evolve?}

\subsection{1 hunchback in Drosophila}

hunchback codes for a $\mathrm{C}_{2} \mathrm{H}_{2}$ zinc finger-type transcription factor (Tautz et al., 1987). In addition to its role as a gap gene during early embryogenesis, hunchback is also required during the development of the central nervous system (Grosskortenhaus et al., 2005; Isshiki et al., 2001; Kambadur et al., 1998; Lehmann and Nüsslein-Volhard, 1987a; Novotny et al., 2002). Here, I will focus on hunchback expression and regulation during early embryogenesis. At the onset of zygotic gene activity, hunchback protein is expressed throughout the anterior half of the embryo while being repressed in the posterior half (Tautz, 1988). In the anterior half, Hunchback is required to initiate development of the head and thorax: the cuticle patterns of mutant embryos devoid of any hunchback protein display a mirror image of abdominal segments in the anterior half of the embryo (Lehmann and Nüsslein-Volhard, 1987a). In the posterior half, the absence of Hunchback is required to allow for the development of a segmented abdomen: if Hunchback is prematurely expressed in the posterior half of the blastoderm, abdominal segmentation is severely affected or completely missing (Hülskamp et al., 1989; Struhl, 1989). Possibly because of its critical role in Drosophila patterning, hunchback is regulated, in part redundantly, by both the posterior and the anterior maternal systems (reviewed in Dearden and Akam, 1999).

\subsection{2 hunchback regulation in Drosophila}

hunchback is transcribed from two different promoters (Tautz et al., 1987); but both transcripts produce the same protein (Tautz, 1988; Tautz et al., 1987). During oogenesis, hunchback is transcribed from its distal promoter (P1) (Schröder et al., 1988; Tautz et al., 
1987), and the P1 transcripts are evenly loaded into the egg (Margolis et al., 1994; Tautz et al., 1987). This maternal expression is driven by an enhancer that is located close to P1 (Lukowitz et al., 1994; Margolis et al., 1994). Nanos, together with Pumilio (Lehmann and NüssleinVolhard, 1987b; Macdonald, 1992), is required to repress translation of the uniformly distributed maternal hunchback mRNA in the posterior half of the embryo (Hülskamp et al., 1989; Irish et al., 1989; Struhl, 1989; Tautz, 1988). Sequences in the 3' untranslated region (UTR) of the hunchback mRNA (Nanos response elements: NREs) have been shown to recruit a complex with Nanos and Pumilio and thereby mediate the translational repression (Murata and Wharton, 1995; Sonoda and Wharton, 1999; Wharton and Struhl, 1991). As a result, maternal hunchback transcripts are translated only in the anterior half of the embryo and are degraded in the posterior half (Tautz, 1988; Tautz and Pfeifle, 1989).

At the onset of zygotic transcription, hunchback is transcribed from its proximal promoter (P2) (Driever and Nüsslein-Volhard, 1989; Schröder et al., 1988; Struhl et al., 1989; Tautz et al., 1987), resulting in strongly increased Hunchback levels throughout the anterior half of the embryo (Schröder et al., 1988; Tautz et al., 1987). This early zygotic hunchback expression is driven by a Bicoid-binding enhancer (Driever and Nüsslein-Volhard, 1989; Driever et al., 1989; Schröder et al., 1988; Struhl et al., 1989), which is about 250 bp long and located immediately upstream of P2 (Driever and Nüsslein-Volhard, 1989; Driever et al., 1989; Struhl et al., 1989). Although Bicoid is required, it appears to be not sufficient to drive hunchback expression throughout the anterior half of the embryo (Simpson-Brose et al., 1994): in mutant embryos that lack functional hunchback protein, expression of hunchback mRNA is restricted to the anterior-most $20 \%$ of the embryo (Simpson-Brose et al., 1994), indicating that Hunchback activates its own transcription synergistically with Bicoid (SimpsonBrose et al., 1994). This interpretation is supported by the presence of a Hunchback-binding site in the minimal Bicoid-binding enhancer (Treisman and Desplan, 1989). Whether, in addition to Bicoid and Hunchback, additional factors are required to sharpen the posterior boundary of early zygotic hunchback expression is the subject of a current debate: staufen (Schüpbach and Wieschaus, 1986; St Johnston et al., 1991), which is required to anchor bicoid transcripts to the anterior pole (St Johnston et al., 1989) and to localize oskar transcripts to the posterior pole (Ephrussi et al., 1991; Kim-Ha et al., 1991), has been suggested to regulate the 
posterior hunchback boundary independent of bicoid (Houchmandzadeh et al., 2002), but this hypothesis has been called into question again by a recent study (Crauk and Dostatni, 2005).

Shortly before the onset of gastrulation, a second zygotic hunchback enhancer located upstream of P1 drives the expression of P1 and P2 transcripts in two circumferential stripes (Lukowitz et al., 1994; Margolis et al., 1995). The anterior stripe is expressed in the presumptive thorax (parasegment four), and the posterior stripe is expressed in the presumptive abdomen (parasegment 13) (Lukowitz et al., 1994; Margolis et al., 1995; Schröder et al., 1988; Tautz et al., 1987). This posterior stripe is under the control of the terminal system and directly activated by the terminal gap gene tailless (Margolis et al., 1995).

Both the maternal and the early zygotic regulation of hunchback are to a certain degree redundant. Maternal hunchback expression is not essential for development (Lehmann and Nüsslein-Volhard, 1987a): mutants without maternal hunchback are viable and do not display a distinct phenotype (Lehmann and Nüsslein-Volhard, 1987a). However, maternal hunchback expression can partly compensate for the loss of zygotic, Bicoid-dependent Hunchback contribution: dependent on the dose of maternal hunchback, the zygotic hunchback phenotype can be partly rescued (Lehmann and Nüsslein-Volhard, 1987a; Wimmer et al., 2000). In the absence of Bicoid-dependent hunchback activation, high amounts of maternal hunchback (four copies) can, in combination with a reduction of the hunchback repressor knirps (one copy), rescue all thoracic segments (Wimmer et al., 2000). Head segments, however, are not rescued in the absence of bicoid activity (Wimmer et al., 2000), indicating that even higher hunchback levels or Bicoid-targets other than hunchback are required for this body part (Wimmer et al., 2000).

\subsection{3 hunchback regulation in dipterans and other insects}

The early zygotic expression of hunchback throughout the anterior half of the embryo is highly conserved in dipterans (Bonneton et al., 1997; Goltsev et al., 2004a; McGregor et al., 2001a; Rohr et al., 1999; Sommer and Tautz, 1991; Stauber et al., 2000; Treier et al., 1989). Within Cyclorrhapha, regulation of hunchback expression was investigated in Drosophila virilis, Musca domestica, Calliphora vicina, and Lucilia sericata (Bonneton et al., 1997; Lukowitz et al., 1994; McGregor; McGregor et al., 2001b; Shaw et al., 2001). A bicoid homo- 
logue has been identified from all four flies (MacDonald, 1990; Schröder and Sander, 1993; Shaw et al., 2001; Sommer and Tautz, 1991), and Bicoid-binding sites in the regulatory DNA of all respective hunchback homologues have been mapped within $800 \mathrm{bp}$ upstream of the putative P2 transcription start sites (Bonneton et al., 1997; Lukowitz et al., 1994; McGregor et al., 2001b). For Drosophila virilis, Musca, and Calliphora, hunchback regulatory DNA including these mapped Bicoid-binding sites has also been analyzed in transgenic Drosophila embryos, and hunchback regulatory DNA of all three species drives reporter gene expression throughout the anterior half of Drosophila blastoderm embryos (Bonneton et al., 1997; Lukowitz et al., 1994; McGregor). Knockdown of hunchback by RNA interference (RNAi) in Musca and Megaselia abdita also suggests a conserved function of early zygotic Hunchback among dipterans (McGregor et al., 2001b; Stauber et al., 2000).

However, it is currently unclear how the anterior domain of hunchback expression is established in non-cyclorrhaphan dipterans and other insects without a bicoid homologue (for a recent review, see Liu and Kaufman, 2005; Stauber et al., 2002). Several lines of evidence suggest that in non-cyclorrhaphan dipterans, a gene with properties very similar to bicoid is responsible for hunchback activation and thus anterior patterning. In the non-cyclorrhaphan dipterans Chironomus spec., Smittia spec., and Bradysia tritici, a symmetrical double abdomen, reminiscent of a combined loss of bicoid and hunchback in Drosophila (Hülskamp et al., 1990), can be induced by UV ablation of the anterior cortex (Kalthoff, 1983; Kalthoff and Sander, 1968; Perondini et al., 1987; Yajima, 1964). In Smittia, this double abdomen phenotype has also been induced by removal of anterior cytoplasm (Schmidt et al., 1975) and by applying RNase to the anterior pole (Kandler-Singer and Kalthoff, 1976), while in Chironomus, the UV induced double abdomen has been reportedly rescued by fractions of poly $(\mathrm{A})^{+} \mathrm{RNA}$ (Elbetieha and Kalthoff, 1988). These and additional experiments in other insects led to the prediction that a localized transcript is essential for patterning the anterior of all dipterans and possibly other insects (reviewed by Kalthoff, 1979; Kalthoff, 1983; reviewed by Sander, 1976).

Recent studies have suggested that orthodenticle (Finkelstein et al., 1990), an evolutionarily conserved Hox gene (reviewed by Reichert and Simeone, 1999), acts synergistically with hunchback to partially substitute for the anterior determinant bicoid in the flour beetle 
Tribolium castaneum and the jewel wasp Nasonia vitripennis (Lynch et al., 2006; Schröder, 2003). If orthodenticle and hunchback are depleted by RNAi, Tribolium and Nasonia embryos, lack head, thorax, and most abdominal segments (Lynch et al., 2006; Schröder, 2003). Orthodenticle, like Bicoid, carries a lysine at position 50 of its homeodomain (Finkelstein et al., 1990). In Bicoid, this residue is critical for the selective binding of the protein to its natural enhancer targets (Hanes and Brent, 1989; Hanes et al., 1994; Treisman et al., 1989). Most homeodomain proteins carry a glutamine at this position (reviewed by Gehring et al., 1994) and differ significantly from Bicoid in their DNA-binding affinities (Hanes and Brent, 1989; Treisman et al., 1989). In Nasonia, maternal orthodenticle transcripts are localized to the anterior pole, similar to bicoid transcripts in Drosophila (Lynch et al., 2006). In Tribolium, maternal orthodenticle transcripts are evenly distributed in the embryo (Li et al., 1996), but translation is repressed in the posterior so that the protein is expressed in an anterior to posterior gradient (Schröder, 2003). Thus, it seems possible that Bicoid substitutes for maternal orthodenticle activity. In Nasonia, however, zygotic hunchback of Nasonia is still expressed in the anterior third of embryos that have been depleted of orthodenticle activity by parental RNAi (Bucher et al., 2002; Lynch et al., 2006), and in Anopheles orthodenticle is not expressed maternally (Goltsev et al., 2004a). Thus, unlike bicoid, orthodenticle is most likely not a primary (Anopheles) or not the only primary (Nasonia) determinant responsible for anterior zygotic hunchback activation.

An alternative model, based on Nanos-mediated translational repression of maternal hunchback transcripts in Drosophila, could explain anterior hunchback expression without anterior input (Curtis et al., 1995; Irish et al., 1989; Simpson-Brose et al., 1994). Enrichment of nanos transcripts at the posterior pole is conserved throughout Diptera (Calvo et al., 2005; Curtis et al., 1995), dipteran nanos homologues can substitute for nanos function in Drosophila (Curtis et al., 1995), and conserved NRE sequences have been identified in the 3' UTRs of hunchback homologues from Tribolium (Wolff et al., 1995), Nasonia (Pultz et al., 2005), and the grasshoppers Schistocerca americana and Locusta migratoria (Patel et al., 2001). Thus, Nanos-dependent translational repression of maternal hunchback transcripts in the posterior half of the embryo might be conserved in many insects (Curtis et al., 1995). Maternal hunchback activity in the anterior half of the embryo may then initiate an auto-regulatory loop, 
which would explain zygotic up-regulation of hunchback in the anterior of lower dipterans (Curtis et al., 1995; Simpson-Brose et al., 1994).

In addition to Nanos, Caudal has also been suggested as a key regulator of early hunchback expression in insects without bicoid (reviewed in Dearden and Akam, 1999; Liu and Kaufman, 2005). This model is based on the analysis of Tribolium hunchback regulatory sequences in transgenic Drosophila embryos (Wolff et al., 1998). In transgenic Drosophila embryos, hunchback regulatory sequences of Tribolium drive reporter gene expression in a Caudal-dependent manner (Wolff et al., 1998), and, consistently, Caudal-binding sites have been mapped to Tribolium hunchback regulatory DNA (Wolff et al., 1998). Recent functional studies of caudal in Tribolium (Copf et al., 2004) and in the cricket Gryllus bimaculatus (Shinmyo et al., 2005) are consistent with a Caudal-dependent hunchback activation. In both species, knockdown of caudal by RNAi results in embryos with only a few head segments (Copf et al., 2004; Shinmyo et al., 2005). Furthermore, caudal RNAi in Gryllus leads to a significant decrease in hunchback expression and a posterior shift of the expression domain (Shinmyo et al., 2005). Since putative NRE sequences have been identified in the 3' UTR of Tribolium hunchback (Wolff et al., 1995), the studies in Tribolium and Gryllus suggests a regulatory mechanism, where hunchback transcription is activated via Caudal and translation of the mRNA is repressed by Nanos (Wolff et al., 1998).

However, the Nanos/Caudal models do not explain how early zygotic hunchback expression in Tribolium is activated in the serosal anlage, since the onset of this expression is independent of caudal (Wolff et al., 1998; Wolff et al., 1995). Furthermore, in Anopheles neither hunchback nor caudal appear to be maternally expressed (Goltsev et al., 2004a). Both taxa, therefore, apparently use alternative means for hunchback regulation. Thus, currently available data strongly suggest that an unidentified anterior maternal system regulates hunchback expression in non-cyclorrhaphan dipterans and possibly in other insects.

\subsubsection{Complementary approaches to explore the evolution of hunchback regulation}

To explore how and when during dipteran evolution hunchback regulation changed from a Bicoid-independent to a Bicoid-dependent mechanism, I first tested the hypothesis that bicoid emerged at the transition from non-cyclorrhaphan to cyclorrhaphan dipterans. In an at- 
tempt to map the emergence of bicoid to this transition, previous studies have covered a variety of non-cyclorrhaphan dipterans (Stauber et al., 2002), but only one of several families from the basal and most likely paraphyletic ashizans (Phoridae; Figure 1) (Stauber et al., 1999). These studies have been extended to other ashizan families and new bicoid homologues have been identified for Platypeza consobrina (Platypezidae, Figure 1) and Lonchoptera lutea (Lonchopteridae, Figure 1). To extend this screen to the predicted anterior determinant of noncyclorrhaphan dipterans, I developed a new screening method for anterior localized transcripts and explored the non-cyclorrhaphan Clogmia albipunctata (Psychodidae, Figure 1). This direct approach failed to identify an anterior localized transcript in Clogmia, and a bicoid homologue in the cyclorrhaphan Episyrphus balteatus (Syrphidae, Figure 1). Thus, to complement this quest for the potential hunchback activator in non-cyclorrhaphan dipterans and Episyrphus, I have also generated reporter constructs to directly compare the early regulation of dipteran hunchback homologues in transgenic Drosophila. This approach also extends previous work, in which the regulation of hunchback homologues from Drosophila virilis, Musca, Calliphora, and Tribolium had been studied in transgenic Drosophila embryos (Bonneton et al., 1997; Lukowitz et al., 1994; McGregor; Wolff et al., 1998). In this way, I studied the hunchback homologues from four basal cyclorrhaphans (Episyrphus, Megaselia, Platypeza and Lonchoptera), and four non-cyclorrhaphan dipterans (Empis livida, Haematopota pluvialis, Clogmia, and Anopheles), which represent mostly paraphyletic dipteran branches (Figure 1). The results provide additional support for Bicoid-dependent hunchback regulation in flies with bicoid, they provide support for Bicoid-independent hunchback regulation in non-cyclorrhaphan dipterans, and they also support for the initial observation that Episyrphus might not contain a bicoid homologue. This unexpected and peculiar position of Episyrphus among cyclorrhaphans was further explored by studying expression of a set of early patterning genes. Also in these expression analyses, Episyrphus displayed intermediate characters between non-cyclorrhaphan and cyclorrhaphan dipterans. 


\section{Material and Methods}

\subsection{Fly culture and egg collection}

Megaselia abdita Schmitz (Phoridae; scuttle or humpbacked flies) were reared as described (Schmidt-Ott et al., 1994) with modifications: The generation time at $25{ }^{\circ} \mathrm{C}$ with a 14/10-hour light/dark cycle was 18-20 days. The flies were reared in plastic stock bottles (diameter: $5.5 \mathrm{~cm}$, height: $13 \mathrm{~cm}$, Genesee) on wet cotton sprinkled with $4-5$ grams of crushed aquarium fish food (Aquatic EcoSystems, Spirulina Flake) per bottle. For egg collection, adults (2500-3000) were placed in a cylindrical Plexiglas cage (diameter: $8 \mathrm{~cm}$, height: 10 $\mathrm{cm})$. Prior to collecting eggs, adult flies were starved on a water-agar plate for the duration of a light cycle. Eggs were collected on moistened filter paper supplemented with a streak of moistened fish food. A peak in egg deposition was observed shortly after the beginning of the dark cycle. Clogmia albipunctata Williston (Psychodidae; moth flies) were reared as described (Schmidt-Ott et al., 1994). The generation time at $25{ }^{\circ} \mathrm{C}$ with a $14 / 10$-hour light/dark cycle was 22-26 days. Eggs were collected as described (Schmidt-Ott et al., 1994). Adults of Episyrphus balteatus Degeer (Syrphidae; hover flies) were collected in the surroundings of Göttingen (Germany); embryos were obtained from P. Katz (Katz Biotech AG, Baruth, Germany). Platypeza consobrina Zetterstedt (Platypezidae; flat-footed flies), Lonchoptera lutea Panzer (Lonchopteridae; pointed-wing flies), Empis livida L. (Empididae; dance flies), and Haematopota pluvialis L. (Tabanidae; horse flies) were collected in the surroundings of Göttingen. Females of Anopheles gambiae Giles, PEST strain (Culicidae; African malaria mosquitoe) were a gift from Frank H. Collins (University of Notre Dame, IN, USA). Drosophila was of the wild-type Oregon-R strain. Sample specimen of Platypeza consobrina larvae were classified by Peter Chandler (Slough, UK), samples of adult Lonchoptera lutea were classified by Urs Schmidt-Ott according to Smith (1969), and samples of adult Empis livida and Haematopota pluvialis were classified by Andreas Stark (Halle, Germany) and Marcel Leclercq (Beyne Heusay, Belgium), respectively. 


\subsection{Cloning}

\subsubsection{Preparation of genomic phage libraries}

Genomic Lambda-Fix II phage libraries were available for Megaselia, Lonchoptera, Haematopota and Clogmia (Schmidt-Ott, unpublished). For this work genomic Lambda-Fix II libraries (Stratagene) were established for Episyrphus and Platypeza. Several attempts to establish a genomic Lambda-Fix II library for Empis failed for unknown reasons. For the Episyrphus library, genomic DNA was prepared from a single adult female; for the Platypeza library, genomic DNA was prepared from $0.5 \mathrm{ml}$ of larvae. Genomic DNA was isolated by SDS lysis as described (Andres and Thummel, 1994) followed by a digest with DNase-free RNase. To generate genomic fragments of 15-20 kb length, the genomic DNA was digested partially by $\mathrm{MboI}$ (NEB) for 60 minutes at $37^{\circ} \mathrm{C}$ using 0.03 to 0.05 units of $M b o \mathrm{I}$ per $\mu \mathrm{g}$ DNA. The libraries were constructed according to the Lambda-Fix II library manual using the Gigapack III XL-11 packaging extract (Stratagene). The primary libraries were titered (Episyrphus balteatus: 710'000 primary clones; Platypeza consobrina: 600'000 primary clones) and amplified; aliquots were stored at $-80{ }^{\circ} \mathrm{C}$. For screening, Hybond- $\mathrm{N}^{+}$nylon membranes (Amersham) were used to prepare plaque lifts. Probes were labeled radioactively using the Rediprime II Random Prime Labeling System (Amersham) with the following modifications: prior to the initial denaturation step, the reaction mix was supplemented with random hexanucleotides to a final concentration of $200 \mathrm{nM}$. After the denaturation step, instead of snap cooling the DNA, the hexanucleotides were allowed to anneal at $37{ }^{\circ} \mathrm{C}$ for 5 minutes. These steps significantly increased labeling efficiency for short probes (130-150 bp). Isolated phages were amplified according to the manual, and phage DNA was prepared using the Lambda Midi Kit (Qiagen).

\subsubsection{Preparation of cDNA templates}

cDNA templates were prepared with the SMART RACE cDNA Amplification Kit (Clontech) and the Marathon cDNA Amplification Kit (Clontech), respectively. For cDNA preparation, $120-150 \mu \mathrm{g}$ of total RNA was extracted from 50 to $100 \mu 1$ fly tissue with RNAwiz (Ambion) according to the manual. Poly $\mathrm{A}^{+}$RNA was enriched using the Oligotex mRNA midi kit (Qiagen), with an average yield of about $2 \mu \mathrm{g}$ poly $\mathrm{A}^{+}$RNA. Enriched poly $\mathrm{A}^{+} \mathrm{RNA}$ was used to prepare the cDNA according to the user manuals. The Anopheles 5' SMART 
RACE cDNA template was prepared from $1.2 \mu \mathrm{g}$ of total RNA, which was isolated from three adult females. The sources of the RNA material and the respective cDNA Amplification Kits used for each of the other species are listed in Table 1 and Table 2, respectively.

\subsubsection{Isolation of homeobox genes}

Platypeza bicoid was amplified by PCR on genomic DNA with the degenerate primer pair 5'-YTGGGYMMAGCYCAGGTSAARATWTGGTT/5'-TYTTBGGYGTYAAHGGYTCRTAGAC, corresponding to positions 367-395 and 805-830 in Megaselia bicoid (GenBank entry AJ133024, Stauber et al., 1999). The product was cloned into pCRII-TOPO (Invitrogen) and sequenced. To obtain Platypeza bicoid cDNA, 5' and 3' rapid amplification of cDNA ends (RACEs) were performed (Table 1), and the products were cloned into pCRII-TOPO. The RACE products did not cover the open reading frame (ORF) completely; therefore, an additional PCR with specific primers was performed on cDNA (Table 1), and the product was cloned into pCR2.1-TOPO. The cDNA sequence of Platypeza bicoid (SEQ01 in the Appendix A.3) is derived from all three clones.

Lonchoptera bicoid was initially isolated from Lonchoptera tristis by PCR on genomic DNA with the degenerate primer pair 5'-TNGTNATGMGNMGNMGNMGNAC/5'CKNCKRTTYTTRAACCA, corresponding to positions 239-260 and 391-407 in Megaselia bicoid. However, due to limited availability of Lonchoptera tristis, Lonchoptera lutea was eventually used in this study. To test for the presence of bicoid in this species, Lonchoptera bicoid was also isolated from Lonchoptera lutea. Using specific primers derived from the bicoid homologue of Lonchoptera tristis, 5' and 3' RACEs were performed (Table 1). The RACE products did not cover the homeobox completely; therefore, an additional PCR with specific primers was performed on cDNA (Table 1). All PCR products were cloned into pCRII-TOPO. The sequence of the Lonchoptera lutea bicoid (SEQ02 in the Appendix A.3) is derived from all three clones.

Episyrphus orthodenticle was amplified by PCR on genomic DNA with the same degenerate primer pair that was used to isolate Lonchoptera bicoid. cDNA was prepared by RACEs, and the products were cloned into pCRII-TOPO (Table 1) and sequenced (SEQ03 in the Appendix A.3). 


\subsubsection{Isolation of hunchback homologues}

hunchback fragments, encoding $133 \mathrm{bp}$ of the conserved first zinc-finger domain (Sommer et al., 1992; Stauber et al., 2000; Tautz et al., 1987), were amplified by PCR from genomic DNA of Platypeza, Lonchoptera, Episyrphus, Empis, and Haematopota as described previously (Stauber et al., 2000). For each of these hunchback homologues, as well as for Megaselia hunchback and Anopheles hunchback, RACEs were performed and cloned into pCRII-TOPO (Table 2). For Episyrphus, Lonchoptera, and Haematopota, respectively, the 3' RACE products did not cover the hunchback ORFs completely; therefore, additional PCRs with specific primers based on cDNA and genomic DNA sequence (Material and Methods, 2.2.6) were performed on cDNA (Table 2), and the products were cloned into pCR2.1-TOPO. Primers to isolate zygotic Megaselia hunchback were designed based on the published sequence of Megaselia hunchback (Stauber et al., 2000); primers to isolate Anopheles hunchback cDNA were designed based on the published genome sequence of Anopheles gambiae (Zdobnov et al., 2002). A cDNA clone of Clogmia hunchback, spanning the entire ORF, was isolated from a maternal Lambda-ZAP cDNA library (Schmidt-Ott, unpublished) using a partial Clogmia hunchback cDNA (Rohr et al., 1999) as a probe (Table 2).

\subsubsection{Isolation of hunchback genomic DNA}

Episyrphus hunchback genomic DNA was isolated by screening a genomic LambdaFix II library, using the 133 bp fragment obtained by degenerate PCR (see Material and Methods 2.2.4) as probe, and by PCR. A phage (Eba-hb ph10) spanning $14 \mathrm{~kb}$ of genomic DNA, including $2.1 \mathrm{~kb}$ upstream of the ORF, was isolated. The region upstream of the ORF, together with $0.9 \mathrm{~kb}$ of the ORF, was amplified by PCR from phage Eba-hb ph10 using a gene-specific primer (5'-CCGACGAGTGTGACTTCCGGTGGGAGTTCAAC) and a T7 primer specific for the phage-internal MCS. The product $(3.0 \mathrm{~kb}$ ) was cloned into pGEM-T Easy (Promega). A second, partially overlapping fragment was amplified by long range PCR from independently prepared genomic DNA using a primer specific for the first exon of the P1 transcript (5'GGGAATATTAATTCTGTAAACGGAGA) and a primer specific for the second exon of the transcript at the beginning of the ORF (5'-CTGCATTGAATCCCAGTTCTGC). This and 
other long range PCRs were performed using TaKaRa La Taq (Takara). The product (5.4 kb) was cloned into pGEM-T Easy, yielding plasmid C616. The genomic Episyrphus hunchback sequence (SEQ07 in the Appendix A.3) is derived from both plasmids and phage Eba-hb ph10. The insert of C616 was cloned as NotI fragment in front of the Drosophila hsp43 basal promotor of the P-element transformation vector pCaSpeR-hsp43-lacZ (Thummel and Pirrotta, 1992), yielding plasmid C681.

Megaselia hunchback genomic DNA was isolated by screening a genomic LambdaFix II library, using a Megaselia hunchback 5' RACE product (Stauber et al., 2000) as probe. A phage (Mab-hb ph2a) spanning $15 \mathrm{~kb}$ of genomic DNA, including $8 \mathrm{~kb}$ upstream of the ORF, was isolated. Two partially overlapping fragments of the phage insert (a $4.5 \mathrm{~kb}$ SpeI-fragment

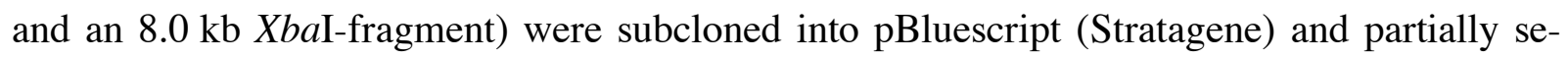
quenced. The genomic Megaselia hunchback sequence (SEQ09 in the Appendix A.3) is derived from both plasmids. The ORF, together with $8 \mathrm{~kb}$ upstream and $1 \mathrm{~kb}$ downstream of the ORF, was amplified by long range PCR from the phage Mab-hb ph2a, using a primer specific to the region 3' of the ORF (5'-CCGTAACATTAACCGTAAC) and a T7 primer specific for the phage-internal multiple cloning site (MCS). The product (11 kb) was cloned into pGEM-T Easy, then excised with NotI and cloned into the NotI site of the P-element transformation vector pCaSpeR 4 (Thummel and Pirrotta, 1992), yielding plasmid C220.

Platypeza hunchback genomic DNA was isolated by screening a genomic LambdaFix II library, using the 133 bp fragment obtained by degenerate PCR (see Material and Methods 2.2.4) as probe. A phage (Pco-hb ph1) spanning $16 \mathrm{~kb}$ of genomic DNA, including $9 \mathrm{~kb}$ upstream of the ORF, was isolated. The phage insert was subcloned into the NotI site of the vector pZErO-1 and partially sequenced (SEQ12 in the Appendix A.3), yielding plasmid C690. $6.2 \mathrm{~kb}$ upstream of the ORF were amplified by long range PCR from plasmid C690, using the primer pair (5'-ATAATCCAGGTGTTGCATCAGG/5'-CTCGTAGCTAGCTGGCTGAAGTGC). The product was cloned into pGEM-T Easy, then excised with NotI and cloned into the NotI site of pCaSpeR-hsp43-lacZ, yielding plasmid C622.

Lonchoptera hunchback genomic DNA was isolated by screening a genomic LambdaFix II library, using the $133 \mathrm{bp}$ fragment obtained by degenerate PCR (see Material and Methods 2.2.4) as probe, and by PCR. A phage (Llu-hb ph2) spanning $16 \mathrm{~kb}$ of genomic DNA, in- 
cluding $1.9 \mathrm{~kb}$ upstream of the ORF, was isolated. The region upstream of the ORF, together with $0.8 \mathrm{~kb}$ of the ORF, was amplified by PCR from phage Llu-hb ph2 using a gene specific primer (5'-CGGCACAACGATACTGATACACAGAAG) and a T3 primer specific for the phage-internal MCS. The product ( $2.7 \mathrm{~kb})$ was cloned into pGEM-T Easy. The phage insert was subcloned into the NotI site of the vector pZErO-1. A third and partially overlapping fragment was amplified by long range PCR from independently prepared genomic DNA using a primer specific for the first exon of the P1 transcript (5'- GACGCGTTCCGATTAACGGATATAA) and a primer specific for the second exon of the transcript immediately upstream of the ORF (5'-TTCAAATTTAACTGCGATGGAGAGC). The product ( $4.6 \mathrm{~kb}$ ) was cloned into pGEM-T Easy, yielding plasmid C514. The genomic Lonchoptera hunchback sequence (SEQ14 in the Appendix A.3) is derived from all three plasmids. The insert of C514 was cloned as NotI fragment into the P-element transformation vector pCaSpeR-hsp43-lacZ, yielding plasmid C515.

Empis hunchback genomic DNA was isolated by long range PCR from genomic DNA using a primer specific for the first exon of the P1 transcript (5'-GTACGCGGGAGTCATGTCTGATGTCTTATA) and a primer specific for the second exon of the transcript at the start of the ORF (5'-ACTATTAATTGCTGTTTGTGGTTCA). The product (6.0 kb) was cloned into pGEM-T Easy and sequenced (SEQ16 in the Appendix A.3). The fragment was then excised with NotI and cloned into the NotI site in front of the minimal even-skipped promotor of the P-element transformation vector pCaSpeR-E2G-lacZ (Markstein et al., 2002), yielding plasmid $\mathrm{C} 681$.

Haematopota hunchback genomic DNA was isolated by screening a genomic LambdaFix II library, using the $133 \mathrm{bp}$ fragment obtained by degenerate PCR (see Material and Methods 2.2.4) as probe. A phage (Hpl-hb phB) spanning $15 \mathrm{~kb}$ of the locus, including $9 \mathrm{~kb}$ upstream of the ORF, was isolated. The phage insert was subcloned into the NotI site of the vector pZErO-1 and sequenced (SEQ18 in the Appendix A.3). $9 \mathrm{~kb}$ upstream of the ORF were amplified by long range PCR on phage Hpl-hb phB using a gene-specific primer (5'- TCCATTGATGGGTATGTTGTAG) and a T7 primer specific for the phage-internal MCS. A smaller fragment $(1.8 \mathrm{~kb})$ comprising the intron sequence of the P1 transcript was amplified by PCR from the same phage using the primer pair (5'-ATTTTGTGAAAATTATGAAATAATTTGGACGC/5'- 
TCCATTGATGGgTATGTTGTAG). Both PCR products were cloned into pGEM-T Easy, then excised with NotI and subcloned in the NotI site of pCaSpeR-hsp43-lacZ, yielding plasmids C423 (9 kb insert; H1) and C688 (1.8 kb insert; H2), respectively.

Clogmia hunchback genomic DNA was isolated by screening a genomic Lambda-Fix II library using a partial Clogmia hunchback cDNA (Rohr et al., 1999) as probe. A phage (Cal-hb ph1) spanning $15 \mathrm{~kb}$ of genomic DNA, including $6.9 \mathrm{~kb}$ upstream of the ORF, was isolated. The region upstream of the ORF, together with $0.7 \mathrm{~kb}$ of the ORF, was amplified by long range PCR from the phage DNA using a gene-specific primer (5'TTGATGTGGATCCTATTGTGCT) and a T7 primer specific for the phage-internal MCS. The product $(7.6 \mathrm{~kb})$ was cloned into pGEM-T Easy, yielding plasmid C213. $6.9 \mathrm{~kb}$ upstream of the ORF were amplified by long range PCR from plasmid C213 using a gene specific primer with an added XhoI site (5'-ATCTCGAGTGACTGAAAGAATAGAAA) and a T7 primer specific for the phage-internal MCS. The product was cloned into pGEM-T Easy, yielding plasmid C214. The insert of C214 was then subcloned as NotI fragment into pCaSpeR-hsp43lacZ, yielding plasmid C215 (K2). In addition, a $4.3 \mathrm{~kb}$ fragment was amplified by long range PCR with specific primers (5'-TGGCTTAGATATAGTCATTACC/5'-ATCTCGAGTGACTGAAAGAATAGAAA) from C213, cloned into pGEM, excised with NotI and cloned into the NotI site of pCaSpeR-hsp43-lacZ, yielding clone C305 (K4). The insert of C305 was then digested with SacII/AgeI, the overhangs were blunted with Mung Bean Nuclease, and the vector was religated yielding clone C305 (K13) with $2.4 \mathrm{~kb}$ of the Clogmia hunchback intron in pCaSpeR-hsp43-lacZ. The genomic Clogmia hunchback sequence (SEQ20 in the Appendix A.3) is derived from plasmids $\mathrm{C} 213$ and $\mathrm{C} 214$.

Genomic DNA of Anopheles hunchback PEST strain was isolated by long range PCR with specific primers (5'-TGTGAGCATTTGCA-TGAGGCTGATTA/5'-CCATCGCCATTACGGAGTCAAAGTTC) based on the sequence of GenBank entry AAAB01008979. The 5.2 $\mathrm{kb}$ fragment, including the intron sequence of the P1 transcript, was cloned into pGEM-T Easy, then excised with NotI and subcloned into the NotI site of pCaSpeR-hsp43-lacZ, yielding plasmid C683. 


\subsection{In situ hybridization, immunocytochemistry and microscopy}

\subsubsection{Embryo fixation}

Embryos were dechorionated and fixed as described (Kosman et al., 2004; Rohr et al., 1999; Stauber et al., 2002), with the following modifications. All embryos were fixed for 25 minutes in $500 \mu 1$ fixation buffer consisting of 50 mM EGTA (pH 8.0), 8\% (Megaselia, Clogmia) or $4 \%$ (Drosophila, Episyrphus) formaldehyde in PBS (137 mM NaCl, $2.7 \mathrm{mM} \mathrm{KCl,} 10$

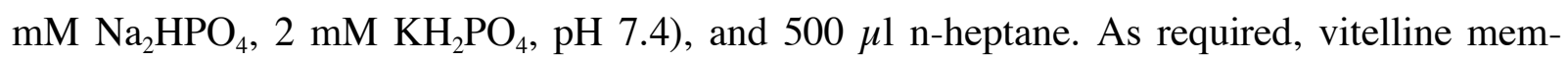
branes were manually removed using a pair of tungsten needles.

\subsubsection{RNA in situ probes}

RNA antisense probes for whole mount in situ hybridization were prepared as described (Lehmann and Tautz, 1994) with modifications. The template vector was linearized at the 5' end of the insert to avoid run-off transcripts, and only RNA probes larger than $1.2 \mathrm{~kb}$ were carbonate-treated (Lehmann and Tautz, 1994). RNA probes were labeled using an NTP mix including either digoxigenin- (DIG), fluorescein- (FITC), or biotin- (BIO) conjugated UTP analogues (Roche) as the substrate for RNA synthesis. The yield of the probe synthesis was determined on an agarose gel in comparison with a DNA standard. The RNA probes used for whole mount in situ hybridization, the UTP analogues used to label each probe, and the templates for the probes are listed in Table 3.

\subsubsection{Whole-mount RNA in situ hybridization}

Whole-mount in situ hybridizations were performed as described (Kosman et al., 2004) with modifications. Postfixation after the xylene washes was omitted. Probes were used at a final concentration of 1-2 $\mathrm{ng} / \mu \mathrm{l}$. For histochemical probe detection, $5 \%$ goat serum in PBT was used as blocking reagent, and alkaline phosphatase conjugated Fab fragments against DIG, FITC or BIOTIN (Roche), depending on the modification of the UTP analogue in the probe. Staining was performed as described (Tautz and Pfeifle, 1989); all embryos of a developmental series were stained equally long. For fluorescent probe detection, FITC-labeled Episyrphus hunchback was detected using a rabbit anti-FITC (1:300 diluted, Molecular Probes) as the primary and an A488 conjugated goat anti-rabbit (1:400, Molecular Probes) as a secondary 
antibody. BIOTIN-labeled Episyrphus zerknüllt was detected using a mouse anti-BIOTIN (1:400, Roche) as the primary and a Cy3 conjugated goat anti-mouse (1:400, Jackson ImmunoResearch) as secondary antibody. The embryos were mounted in $70 \%$ glycerol in PBS, supplemented with 4\% N-propyl gallate for fluorescent microscopy. Confocal scans were taken on a Leica SP2 AOBS Spectral Confocal Microscope. For 3D projection of image stacks ImageJ software (Wayne Rasband, National Institute of Mental Health, Bethesda, Maryland, USA) was used. Images were finished in Photoshop 7 (Adobe).

\section{4 cDNA library from embryonic pole cytoplasm}

\subsection{1 cDNA preparation from pole cytoplasm}

Synthesis and amplification of cDNA from pole cytoplasm were performed as previously described (Brady and Iscove, 1993; Dulac and Axel, 1995; Kramer, 2000) with modifications. Pole cytoplasm was isolated from the embryo using a Narishige XYZ micromanipulator system with a Narishige IM-300 Microinjector (version 8.2A). Needles were prepared from glass capillaries (A-M Systems: 615000; glass, filament, thin-wall, $1.0 \mathrm{~mm}$ x .75 mm, 4") using a Flaming/Browning Micropipette puller (Sutter Instrument: Model P-87) with a trough-style heating element (pulling parameters: pressure: 505; heat: 560; pull: 100; velocity: 40; time: 100). Needles were ground using a Narishige's EG-44 capillary grinder at a speed of 8.0 at $30^{\circ}$ for 40-45 seconds, which produced a pore small enough to allow for control of capillary forces. Prior to use, the needles were UV-irradiated. They were loaded from the tip with approximately $0.1 \mu \mathrm{l}$ of cDNA lysis buffer (1X MMLV buffer [Invitrogen] with 0.5\% NP40 [USB], containing $24 \mu \mathrm{M} \mathrm{pd}(\mathrm{T})_{24}$ [IDT], $0.2 \mathrm{U} / \mu 1$ SuperRNaseIn [Ambion], $0.3 \mathrm{U} / \mu 1$ RNAguard [Amersham], and $20 \mu \mathrm{M}$ each of dATP, dCTP, dGTP, dTTP [Roche]). Approximately $0.5 \%$ of the total egg volume was taken up from anterior cortical egg cytoplasm by carefully releasing the balance pressure. The contents of the entire needle were then cleared from the needle into thin-welled $0.5 \mathrm{ml}$ microfuge tubes (Costar) with $4.5 \mu \mathrm{l}$ of cDNA lysis buffer. The isolated cytoplasm was subsequently dissociated for one minute at $65{ }^{\circ} \mathrm{C}$ followed by an annealing step for the $\mathrm{pd}(\mathrm{T})_{24}$ oligonucleotide at room temperature $\left(22^{\circ} \mathrm{C}\right)$ for 2 minutes. Firststrand cDNA synthesis, terminal transferase, and cDNA amplification were then performed exactly as described (Kramer, 2000). To amplify the cDNA, however, a modified AL1 primer 
was used in order to introduce a NotI site within the linker region (AL1mod: 5'AGCGGCCGCGAATCC $(T)_{24}$ ). To avoid a bias towards smaller transcripts during the PCRbased cDNA amplification, the first strand synthesis conditions were chosen to generate cDNAs of around 100-700 bases regardless of the size of the original RNA template (Brady and Iscove, 1993). Following cDNA amplification, the PCR reactions were stored in aliquots at $-80^{\circ} \mathrm{C}$.

\subsubsection{Preparation of bacterial libraries from amplified cDNA}

The PCR products were size-selected on a $2 \%$ agarose gel (300-1000 bp), eluted with QIAquick Gel Extraction Kit (Qiagen), and ligated into pCRII-TOPO vector. Transformation was performed by electroporation using a MicroPulser (BioRad). Random colonies were hand-picked into 384-well plates (Genetix) and grown overnight in 2YT media supplemented with 7\% glycerol as described (Dunham et al., 1997). Libraries were stored at $-80^{\circ} \mathrm{C}$. The bacterial libraries were spotted in multiple replicates onto Hybond- $\mathrm{N}^{+}$nylon membrane using a QBot spotting robot (Genetix). For the Megaselia library, a low-density spotting scheme (4 twin spots per $3 \times 3 \mathrm{~mm}$ ) was used; for the Clogmia libraries, the spotting density was doubled ( 8 twin spots per $3 \times 3 \mathrm{~mm}$ ). Spotted filters were processed as described (Dunham et al., 1997).

\subsubsection{Hybridization of the libraries and subtractive screening}

For subtractive screening, different filter replicates of a spotted library were hybridized with radioactively labeled cDNA from the anterior and the posterior pole, respectively. cDNA pools were radioactively labeled by PCR with $1 \mu \mathrm{Ci} / \mu 1 \alpha\left[\mathrm{P}^{32}\right] \mathrm{dCTP}$ (Amersham, specific activity: $3000 \mathrm{Ci} / \mathrm{mmol}$ ) exactly as described (Kramer, 2000). Hybridizations were performed in Rapid-hyb buffer (Amersham) at $65^{\circ} \mathrm{C}$ for $2-3$ hours. Excess radioactivity was removed by washing the filters in $0.2 \mathrm{xSC}$ ( $3 \mathrm{mM}$ sodium citrate; $30 \mathrm{mM} \mathrm{NaCl}, \mathrm{pH} 7)$ and $0.1 \%$ SDS at $65^{\circ} \mathrm{C}$ for 30 minutes. Following hybridization, the filter replicates were exposed overnight to Storage Phosphor Screens (Molecular Dynamics). The screens were read with a Storm860 Scanner (Molecular Dynamics). The read-outs were exported as grayscale images using ImageQuant version 1.2 for Macintosh (Molecular Dynamics). Brightness and contrast were uniformly adjusted in Photoshop 7. The images were aligned in Freehand 11 (Macromedia) and 
imported in Photoshop using the red and the green channel of an RGB images for hybridization experiments with anterior and posterior cDNA, respectively. Red signals indicated hybridization predominantly with anterior cDNA and were identified by eye.

\subsubsection{Virtual northern hybridization}

Samples of $10 \mu \mathrm{l}$ of PCR amplified cDNA were separated on a $2 \%$ agarose gel together with the $1 \mathrm{~kb}$ DNA ladder (Invitrogen) as size standard. Separated cDNA was transferred to Hybond- $\mathrm{N}^{+}$nylon membranes (Sambrook and Russel, 2001) and hybridized in Rapid-hyb buffer at $65{ }^{\circ} \mathrm{C}$ for $2-3$ hours to radioactively labeled probes. Probes were labeled radioactively using the Rediprime II Random Prime Labeling System. Nonspecifically bound probe molecules were removed by washing in $0.2 x \mathrm{SSC}$ and $0.1 \% \mathrm{SDS}$ at $65^{\circ} \mathrm{C}$ for 30 minutes.

\section{$3 \quad$ Results}

\subsection{Identification of bicoid orthologues from Platypeza and Lonchoptera}

The presence of a functionally conserved bicoid gene in Megaselia and its apparent absence in the lower dipterans indicates that this activator of zygotic hunchback transcription originated in the stem lineage or early radiation of cyclorrhaphan flies (see Introduction). To more precisely determine the occurrence of bicoid in lower cyclorrhaphans, a PCR-based screen for bicoid orthologues was performed in Lonchoptera, Platypeza, and Episyrphus (Material and Methods 2.2.3). The exact phylogenetic relationship of these taxa has not been firmly established, but they constitute a very broad sample of lower cyclorrhaphans and are probably of paraphyletic origin (Figure 1). For each taxon, amplified homeobox fragments were recovered and compared to the GenBank database (Benson et al., 2006) using the BLAST algorithm (Altschul et al., 1997). The results suggest that the homeobox sequences from Platypeza and Lonchoptera are orthologous to bicoid (data not shown). Homeobox fragments from Episyrphus that were isolated using degenerate bicoid primers proved to be an orthodenticle orthologue (see Results 3.6.3) or a zerknüllt orthologue, which will be described elsewhere (Rafiqi et al., in preparation). Despite multiple attempts, a bicoid-like sequence could 
not be recovered from Episyrphus (see Materials and Methods 2.2.3 for details on the cloning strategy and Results 3.5 for additional evidence supporting the absence of bicoid from Episyrphus). For the putative bicoid homologues of Lonchoptera and Platypeza, the corresponding cDNAs were cloned (Material and Methods 2.2.3), and the predicted homeodomain sequences were aligned with the Bicoid homeodomains of Drosophila and Megaselia (Figure 2). The homeodomains from Lonchoptera and Platypeza carry a lysine (K) at position 50 and an arginine $(\mathrm{R})$ at position 54, which are both characteristic amino acids for Bicoid homeodomains and essential for their binding specificity to nucleic acids (Dave et al., 2000; Hanes and Brent, 1989; Niessing et al., 2000; Treisman et al., 1989). The homeodomains from Lonchoptera and Platypeza share significantly higher similarities with the homeodomains of Drosophila and Megaselia Bicoid than with the homeodomains of Zerknüllt or Orthodenticle (Figure 2). These observations strongly suggest that the newly identified homeobox sequences are orthologous to bicoid. An alignment of the entire open reading frames (Figure 3) reveals sequence conservation not only in the functional homeodomain but also in all additional domains that are known to be required for Bicoid function (reviewed in McGregor, 2005). Together with the functional analysis of Megaselia bicoid (Stauber et al., 2000), this high degree of sequence conservation indicates that the newly identified homologues are also functionally similar to bicoid in Drosophila. Since previous searches for a bicoid orthologue in Anopheles (Zdobnov et al., 2002), Empis, Haematopota and Clogmia (Stauber et al., 2002) were negative, I conclude that of the eight species compared in this study, at least three (Megaselia, Platypeza, Lonchoptera) - and probably only these three - contain bicoid.

\subsection{Subtractive screening for bicoid-like genes: a new method}

To screen Episyrphus and lower dipterans for genes that encode anterior localized transcripts, I developed a new method based on protocols to prepare cDNA libraries from single cells (Brady and Iscove, 1993; Dulac and Axel, 1995; Kramer, 2000). I tested this protocol in Megaselia using Megaselia bicoid as a positive control. cDNA was synthesized and PCR amplified from RNA that was isolated from the anterior and posterior pole cytoplasm of an hourold Megaselia embryo, respectively (Figure 4 A, Materials and Methods 2.4.1). At this intravitteline cleavage stage, the embryo contains only maternal mRNAs. The amplified cDNA 
pools were blotted and analyzed by hybridization against a radioactively labeled bicoid probe (virtual northern, Material and Methods 2.4.4). As expected, Megaselia bicoid cDNA was detected only in cDNA pools prepared from anterior cytoplasm (Figure 4 B). Next, a cDNA library was prepared from the anterior cDNA pool (Material and Methods, 2.4.2). To determine the relative abundance of Megaselia bicoid clones in this library, an estimated 5,000 bacterial clones of the library were hybridized with a labeled Megaselia bicoid probe. 48 of these colonies hybridized to the probe, indicating that approximately one in a hundred clones contain a Megaselia bicoid cDNA (data not shown).

To test whether Megaselia bicoid could be reliably identified by subtractive screening, 1,536 bacterial clones were spotted, grown, and lysed on nylon filters according to a predetermined twin spot scheme (Figure $5 \mathrm{~A}$, Material and Methods 2.4.2). One filter replica of the library was hybridized with a radioactively labeled pool of cDNAs, which had been prepared from posterior cytoplasm. Signals obtained from this hybridization were shaded green. A second filter was hybridized against a pool of cDNA prepared from anterior cytoplasm, and signals resulting from this hybridization were shaded red. Both images were then merged (Figure $5 \mathrm{~B}$ ). In these merged images, green and yellow twin signals indicated hybridization with the posterior cDNA pool, and the corresponding clones were excluded from further analysis. Clones that hybridized only with the anterior cDNA pool can be identified as red twin spots. A sample of 14 clones was sequenced. Of this sample, only the clones with a strong red signal contain Megaselia bicoid (see boxes in Figure 5 B). This result indicates that the subtraction of non-localized transcripts is efficient and provides a filter for undesired but presumably abundant cDNAs from housekeeping genes in each cDNA sample. 'False positive' clones (red twin spots of clones, which do not contain Megaselia bicoid) were not observed. To test for 'false negative' clones (Megaselia bicoid-containing clones which remain hidden in the subtractive screen), a third filter replicate was hybridized with labeled Megaselia bicoid cDNA. This control reveals additional putative Megaselia bicoid clones (Figure $5 \mathrm{C}$ ), indicating that the subtractive screen detects only a subset of the Megaselia bicoid clones. Out of 28 clones positive for Megaselia bicoid (1.8\% of all spotted clones), only four were detected after subtraction, indicating a relatively high rate ( $86 \%)$ of 'false negative' clones. 
The results demonstrate that a gene encoding a strictly anterior localized transcript, such as Megaselia bicoid, can be cloned using the described cDNA subtraction screen. Furthermore, the results suggest that only bicoid transcripts are abundantly localized to the anterior pole of Megaselia embryos.

\subsection{Subtractive screening for bicoid-like genes in Clogmia}

The subtractive screen was designed to screen either for a potentially missed bicoid homologue in Episyrphus (see Results 3.1) or to screen non-cyclorrhaphan dipterans without a bicoid homologue for anterior localized transcripts (see Introduction 1.3.3). Because only the Clogmia culture was readily available then, I decided to test the hypothesis that Clogmia, a non-cyclorrhaphan dipteran (Figure 1), uses localized mRNA as an anterior determinant.

It has been shown that bicoid transcripts diffuse slightly during early development (Stauber et al., 2000). Without knowing where exactly transcripts might be localized in the anterior cortex of Clogmia embryos, I therefore decided to prepare cDNA libraries from anterior poleplasm of Clogmia embryos from two consecutive intravitelline cleavage stages prior to the onset of zygotic transcription. A first cDNA library from anterior pole cytoplasm was prepared from a one hour-old embryo, with the chance to isolate cytoplasm highly enriched in anterior localized transcripts, but with the risk that the wrong portion of cytoplasm would be chosen or that the transcripts might still be too tightly localized to be efficiently removed. A second library was prepared from a three hour-old embryo, with a higher chance of retrieving relevant cytoplasm, but with the risk that the transcripts would be too dilute to be successfully amplified and enriched during the cloning process.

Roughly 14,000 clones of each library were spotted and screened as described for Megaselia. A total of 161 clones were selected and sequenced. Of those clones, $6 \%$ (9 out of 161) contained empty vectors, and $9 \%$ (15/161) contained multiple and therefore unreadable inserts. $45 \%(72 / 161)$ of the clones contained ribosomal subunits or riboproteins, and $17 \%$ (27/161) of the clones contained human genes (Figure 6 A). The remaining 23\% (38/161) of the sequenced clones comprised ten different cDNAs (Figure $6 \mathrm{~B}$ ) which could not be placed into any of the former categories. In order to assess possible functions of these ten cDNAs, their sequences were compared with genes from two virtual GenBank databases (Cummings et 
al., 2002) that either contained only Drosophila genes or all Arthropoda genes (Table 4). Significant support for sequence homology (BLAST Expect values $<0.01$ ) to Drosophila genes was found in six of the ten cDNAs. The corresponding six Drosophila genes are the gene Odorant-binding protein 99a (Obp99a, (Galindo and Smith, 2001)), which encodes a member of a large family of proteins that bind lipophilic odorant molecules (Vogt et al., 1991), the gene exuperantia (exu, (Marcey et al., 1991; Schüpbach and Wieschaus, 1986)), which encodes a protein required for bicoid mRNA localization (Berleth et al., 1988), the putative Histone 3-encoding gene His3:CG31613 (Drysdale et al., 2005), the gene Decondensation factor (Df31, (Crevel and Cotterill, 1995)), encoding a chromatin associated component (Crevel et al., 2001), the gene CG14764 with currently unknown function (Drysdale et al., 2005), and the gene CG1967, encoding a putative p24 protein (Liang and Biggin, 1998), which is involved in intracellular post-golgi transporter activity (reviewed in Carney and Bowen, 2004). Insignificant homology scores to Drosophila genes were obtained for the remaining four of the ten cDNAs (BLAST Expect values $>2.5$ ). The corresponding four Drosophila genes are CG6459, which encodes a putative component of the mitochondrial matrix (Drysdale et al., 2005), jing (Karpen and Spradling, 1992; Liu and Montell, 2001), which encodes a $\mathrm{C}_{2} \mathrm{H}_{2}$ zinc finger transcription factor (Liu and Montell, 2001), the Amylase distal gene (Amy-d (Boer and Hickey, 1986)), and the Graf gene (Drysdale et al., 2005), which encodes a product with putative Rho GTPase activator activity (Drysdale et al., 2005).

Based on their functions and their high similarity to Anopheles homologues, seven cDNAs were selected for further analyses (Table 4). To test whether these cDNAs were enriched in the anterior pole of the embryo, the expression profiles of Obp99a like (Figure 7 A), CG14761 like (Figure 7 B), Df31 like (Figure 7 C), exu like (Figure 7 D), jing like (Figure 7 E), CG6459 like (Figure 7 F), and CG1967 like (Figure 7 G), respectively, were analyzed in virtual northern blots. A radioactively labeled probe of each cDNA was hybridized to pools of amplified cDNA from anterior and posterior cytoplasm of four Clogmia embryos (Material and Methods 2.4.4). None of the seven cDNAs always and exclusively hybridized with the cDNA pools from anterior poleplasm. Six of the analyzed cDNAs were excluded from further analyses because they hybridized to amplified cDNA from anterior and posterior cytoplasm. CG6459 like hybridized exclusively to the anterior fraction of one preparation but not at all to the cDNA pools 
from other embryos. Expression of this candidate was tested by whole-mount in situ hybridization of Clogmia embryos. Using an antisense RNA probe of CG6459 like, staining was detected weakly but ubiquitously throughout the embryo; using a respective sense probe of CG6459 like, staining was not observed (data not shown).

Although most cDNAs hybridized to amplified cDNA from both anterior and posterior cytoplasm, some gave a stronger signal with the cDNA pool from which the library was made (e.g. jing like, Figure 7 E, embryo 3; or CG1967 like, Figure 7 G, embryo 1). These observations suggest that the cDNAs of evenly distributed transcripts may be under- or overrepresented in individual cDNA pools. Such biologically insignificant artifacts could be caused by a bias in the amount of removed cytoplasm or PCR amplification of cDNAs after reverse transcription. Together, the results suggest that Clogmia might lack abundant, strictly localized maternal transcripts at the anterior pole of early developing eggs.

\subsection{Cloning of dipteran hunchback genes}

The restricted occurrence of bicoid in dipterans suggests differences in early hunchback regulation between Episyrphus and lower dipterans on one hand and other cyclorrhaphans on the other. To test this hypothesis, I compared the regulation of eight dipteran hunchback homologues in transgenic Drosophila embryos. For these investigations, hunchback cDNA containing 5' UTR with putative leader sequence was mapped onto genomic DNA. cDNAs including 5' UTRs were newly isolated from Episyrphus, as well as from Megaselia, Lonchoptera, Platypeza, Empis, Haematopota, Clogmia, and Anopheles (Table 2 and Materials and Methods 2.2.5). Genomic hunchback DNA was isolated from Episyrphus, Megaselia, Platypeza, Lonchoptera, Empis, Haematopota, and Clogmia, employing genomic phage libraries and/or PCR on genomic DNA (Materials and Methods 2.2.6). Genomic DNA sequence of Anopheles hunchback was obtained directly from the sequenced Anopheles genome (Holt et $a l ., 2002)$. Protein trees based on the predicted amino acid sequences of the N-terminal zinc finger domain (amino acids 243-349 in the Drosophila protein, Figure 8) together with the alignment of the predicted amino acid sequences of the entire open reading frames (Figure 9) strongly suggest that the newly identified genes are hunchback orthologues. The alignment reveals sequence conservation not only in the functional zinc finger domains but also in sev- 
eral additional motifs that are thought to be specific for the hunchback protein, such as the A-, C-, D-, E, and F-boxes, the molecular functions of which, however, are still unknown (Hülskamp et al., 1994; McGregor et al., 2001a; Tautz et al., 1987; Figure 9).

In Megaselia, a second transcript was isolated in addition to the previously identified maternal transcript (Stauber et al., 2000). Both transcripts differ in their first exon. The maternal transcript derives from the distal promotor (P1, Figure 10); the newly identified transcript isolated from early embryos is probably zygotic (see Material and Methods 2.2.5) and derives from the proximal promotor (P2, Figure 10). These findings suggest that the genomic organization of hunchback is conserved between Megaselia and Drosophila and that the P1 and P2 transcripts of both species are directly homologous. Two alternative transcripts with differing 5' UTRs were also identified in Platypeza (Figure 10). These splicing variants, however, were obtained from larval tissue (embryos were not available), and it is unclear whether they are homologues to the maternal and zygotic hunchback transcripts of Drosophila and Megaselia. In Lonchoptera, a single maternal transcript was detected in adult females (embryos were not available; Figure 10). In Episyrphus, three splice variants with alternative 5' UTRs were identified from pools of 0-5 hours old embryos (Figure 10). In all lower dipterans (Empis, Haematopota, Clogmia, Anopheles), only one splice variant was isolated (Figure 10). In Empis, the occurrence of only a single splice variant was confirmed by comparing cDNAs that were isolated from ovarian and embryonic cDNA templates. In Clogmia, the occurrence of only a single splice variant was confirmed by comparing cDNAs from 0-2 hour-old and 5-6 hour-old embryonic libraries (onset of zygotic transcription at about 4 hours of development) and by developmental Northern analysis (Prell and Schmidt-Ott, unpublished; supplemental Figure S1). Together, the data suggest that, unlike cyclorrhaphans, non-cyclorrhaphan dipterans use the same hunchback splice variant during oogenesis and early embryogenesis.

In the putative 3' UTRs of Megaselia, Platypeza, Lonchoptera, Episyrphus, Haematopota and Clogmia hunchback sequences, I identified putative NRE sequences (Figure 11), which all reside within $0.6 \mathrm{~kb}$ downstream of the ORF (Figure 10). The presence or absence of NRE sequences in Empis could not be determined due to limited sequence information. In Anopheles, NRE sequences could not be identified within $8.0 \mathrm{~kb}$ downstream of the ORF, which is consistent with the reported absence of maternal hunchback expression in this species 
(Goltsev et al., 2004a). These findings support previous studies, which suggested that translational repression of maternal hunchback by Nanos is conserved in dipterans (Curtis et al., 1995).

\subsection{Functional comparison of early dipteran hunchback regulation}

To functionally compare the transcriptional regulation of the hunchback homologues, I cloned reporter constructs with putative regulatory DNA of each hunchback homologue (Figure 10, Table 5) and compared their expression in transgenic Drosophila embryos (Figure 12). In the case of Megaselia, the entire hunchback locus was tested. All other constructs include a strong basal Drosophila promoter (eve or hs43, respectively) and the lacZ gene as reporter (see Materials and Methods 2.2.6). For each construct, two to four independent stable transgenic Drosophila lines were established by P-element mediated germline transformation (Rubin and Spradling, 1982; Table 5). The transgenic expression patterns of the reporter genes were compared to endogenous hunchback expression in Drosophila (Figure 12 A, B; Tautz et al., 1987), Megaselia (Figure 12 C, D; Stauber et al., 2000), and Clogmia (Figure 12 E, F; Rohr et al., 1999).

At the onset of blastoderm cellularization, the reporter of the Megaselia construct is activated in the anterior half of the embryo. During cellularization, anterior reporter expression disappears from the anterior-most portion of the embryo, while a new domain appears at the posterior pole (Figure $12 \mathrm{G}, \mathrm{H}$ ). This pattern resembles the endogenous expression of hunchback in Drosophila and Megaselia (Figure 12 A-D).

The Platypeza construct is also expressed in the anterior half of syncytial blastoderm embryos, excluding, however, the anterior $20 \%$ of the embryo. During cellularization, this expression extends ventrally towards the anterior pole, and reporter expression also appears at the posterior pole of the embryo (Figure $12 \mathrm{I}, \mathrm{J}$ ).

The Lonchoptera construct drives reporter expression in an anterior stripe from $90-75 \%$ EL (egg length; $0 \%$ at the posterior pole) in syncytial blastoderm embryos but, unlike the Megaselia and Platypeza constructs, expression during cellularization is less dynamic and is absent in the posterior half of the embryo (Figure $12 \mathrm{~K}, \mathrm{~L}$ ). 
The Episyrphus construct, in contrast to the other cyclorrhaphan constructs, is expressed exclusively in the posterior half of the syncytial blastoderm embryo. During cellularization, the expression disappears from the posterior pole, resulting in a broad stripe of expression from 50-20\% EL (Figure 12, M, N). Thus, this reporter expression is roughly complementary to the endogenous early hunchback expression of Drosophila (Figure 12 A, B).

For Haematopota, two constructs were analyzed. The larger construct includes $9 \mathrm{~kb}$ of genomic DNA upstream of the ORF (H1). This construct initially drives posterior reporter gene expression in the syncytial blastoderm, which is then cleared from the posterior pole (Figure $12 \mathrm{O}, \mathrm{P}$ ) during cellularization. This expression is similar to both the early expression of the Episyrphus construct (Figure $12 \mathrm{M}, \mathrm{N}$ ) and the expression of a comparable Tribolium construct (Wolff et al., 1998). Subsequently, the Haematopota construct is also expressed in a weak anterior stripe, which appears as a second domain towards the end of cellularization (Figure 12 P). Similar expression has also been reported for the Tribolium construct (Wolff et al., 1998). A shorter Haematopota construct which included only the $1.8 \mathrm{~kb}$ intron sequence of Haematopota hunchback (H2) was not expressed in pregastrular embryos (data not shown).

For Clogmia, both a larger construct (K2), comprising $6.9 \mathrm{~kb}$ upstream of the ORF, and a shorter construct (K13), spanning only intron sequence, drive reporter gene expression in a dorsal domain and in a weak transverse stripe at 65-60 \% EL of the blastoderm and subsequently in the developing amnioserosa of the gastrulating embryo (Figure 12 Q, R). Expression of these constructs in the dorsal blastoderm and the amnioserosa is reminiscent of the endogenous extraembryonic expression of Clogmia hunchback shortly before the onset of gastrulation (Figure $12 \mathrm{E}, \mathrm{F})$.

The constructs with genomic DNA from Empis hunchback and Anopheles hunchback (Figure 10) are not expressed in pregastrular Drosophila embryos (data not shown).

Although the results of these enhancer analyses are heterogeneous, at least two aspects deserve attention. First, reporter expression of the Megaselia and Platypeza constructs in transgenic Drosophila is similar to endogenous hunchback expression patterns in Drosophila (Tautz et al., 1987) and Megaselia (Stauber et al., 2000). This finding does not exclude the possibility of substitutions among hunchback regulators between these species, but it is more parsimonious to explain the results with an essentially conserved regulatory network for early 
hunchback activation between Drosophila, Megaselia, and Platypeza. This conclusion is supported by RNAi knockdown of bicoid in Megaselia, which causes a duplicated posterior hunchback expression at the anterior pole (Figure 13), and by the presence of Bicoid-binding sites within the P1 intron upstream of P2 of Megaselia hunchback (Shaw and Schmidt-Ott, unpublished; supplemental Figure S3). The Lonchoptera data are consistent with this hypothesis but more difficult to interpret because expression in an anterior head stripe may occur as an artifact (Klingler et al., 1996). The second finding of special interest is that expression of the Episyrphus construct is confined to the posterior blastoderm. The expression of this construct is significantly different from the expression of all other cyclorrhaphan reporter constructs and resembles the posterior expression patterns that were obtained with the Haematopota and Tribolium constructs (Wolff et al., 1998). This raises the question, whether these species use, at least in part, a similar Bicoid-independent mechanism of hunchback regulation. The mechanism could be dependent on the transcription factor Caudal as has been suggested previously for Tribolium (Wolff et al., 1998).

\subsection{Expression studies in Episyrphus}

To explore whether Episyrphus, besides a putative lack of Bicoid-dependent hunchback regulation, also shares characteristics in early pattern formation with non-cyclorrhaphan dipterans and other holometabolous insects, I decided to study the expression of Episyrphus hunchback, zerknüllt as a marker for extraembryonic tissue, and orthodenticle as a potential alternative to Bicoid as hunchback activator. The results suggest that early pattern formation in Episyrphus is a mosaic of pattern formation in cyclorrhaphan and non-cyclorrhaphan dipterans.

\subsubsection{Episyrphus hunchback shares expression characteristics of cyclorrhaphan} and non-cyclorrhaphan dipterans

Cyclorrhaphans express hunchback in a posterior domain (Bonneton et al., 1997; McGregor et al., 2001a; Sommer and Tautz, 1991; Stauber et al., 2000; Tautz et al., 1987; Treier et al., 1989), while only lower dipterans express hunchback in the extraembryonic blastoderm (Goltsev et al., 2004a; Rohr et al., 1999). The loss of this expression in cyclorrhaphan 
dipterans correlates with the occurrence of Bicoid (Berleth et al., 1988; Gregor et al., 2005; Schröder and Sander, 1993; Seeger and Kaufman, 1990; Shaw et al., 2001; Sommer and Tautz, 1991). I studied the expression of Episyrphus hunchback throughout early development and compared it to both lower and higher dipterans. In the freshly laid egg, the maternal transcripts of Episyrphus hunchback are evenly distributed (Figure 14 A) but disappear from the posterior half during blastoderm formation (Figure 14 B, C). A distinct increase of Episyrphus hunchback expression throughout the anterior half of the syncytial blastoderm embryo marks the onset of zygotic expression (Figure 14 D). During cellularization, a second expression domain appears at the posterior pole (Figure $14 \mathrm{E}$ ), and expression in the anterior half resolves into a prominent stripe from about $60-55 \% \mathrm{EL}$ (Figure $14 \mathrm{E}, \mathrm{F}$ ). This pattern closely resembles hunchback expression in other cyclorrhaphans (Figure 12 A-D; Bonneton et al., 1997; McGregor et al., 2001a; Sommer and Tautz, 1991; Stauber et al., 2000; Tautz et al., 1987; Treier et al., 1989), but differs from pregastrular hunchback expression in lower dipterans, which lack the posterior domain (Figure 12 E, F; Goltsev et al., 2004a; Rohr et al., 1999). However, Episyrphus hunchback is also expressed in a mid-dorsal stripe of the blastoderm, which expands from anterior to posterior (Figure $14 \mathrm{G}-\mathrm{L}$ ). Dorsal hunchback expression is absent in other cyclorrhaphans but reminiscent of hunchback expression in the extraembryonic anlage of lower dipterans (Figure 12 E, F; Goltsev et al., 2004a; Rohr et al., 1999), Tribolium (Wolff et al., 1995), and Nasonia (Pultz et al., 2005). With the onset of gastrulation, the dorsal expression of Episyrphus hunchback broadens and transcripts are predominantly detected in a narrow band along the margins of the extraembryonic primordium (Figure 14 I, L). Similar expression dynamics have been reported for Tribolium hunchback (Wolff et al., 1995). Thus, Episyrphus hunchback expression in the early embryo shares characteristics specific for cyclorrhaphans (posterior expression domain), for lower dipterans (dorsal/extraembryonic expression domain), and with all dipterans it shares early expression in an anterior cap.

\subsubsection{The extraembryonic anlage of Episyrphus extends to the anterior pole}

To further explore the hypothesis that Episyrphus hunchback is expressed in the extraembryonic anlage, I compared the dorsal hunchback expression with the expression of zerknüllt, a conserved marker for the extraembryonic anlage (Falciani et al., 1996). During the 
onset of gastrulation, dorsal hunchback expression (Figure $15 \mathrm{~A}, \mathrm{~B}$ ) and zerknüllt expression (Figure 15 C, D) perfectly overlap (Figure 15 E, F). This result not only indicates that Episyrphus hunchback is expressed in the extraembryonic anlage, but it also suggests that in Episyrphus the extraembryonic anlage extends to the anterior pole. In Drosophila and Megaselia, both of which use bicoid as an anterior determinant, the extraembryonic anlage is restricted to the dorsal-most blastoderm while the anterior blastoderm gives rise to embryonic structures (Campos-Ortega and Hartenstein, 1997; Rushlow and Levine, 1990; Stauber et al., 1999). In many insects that lack bicoid, the extraembryonic (serosal) primordium extends to the anterior tip of the blastoderm (e.g. Clogmia (Rohr et al., 1999), the honey bee Apis mellifera (Fleig and Sander, 1988), and Tribolium (Wolff et al., 1995)).

To test whether the extraembryonic anlage of Episyrphus is structurally more closely related to non-cyclorrhaphan dipterans than to Drosophila, Episyrphus zerknüllt expression was further analyzed. In pre-blastoderm embryos, Episyrphus zerknüllt transcripts could not be detected by whole-mount in situ hybridization (Figure $16 \mathrm{~A}$ ), suggesting that, like in other cyclorrhaphans, zerknüllt is not maternally expressed in Episyrphus. During the early blastoderm stage, zygotic transcripts appear in a broad dorsal domain with an enrichment of transcripts at the anterior pole (Figure $16 \mathrm{~B}$ ). During cellularization of the blastoderm, anterior zerknüllt expression extends in a mid-dorsal stripe, while all other expression disappears (Figure $16 \mathrm{C}-\mathrm{I})$. At the onset of gastrulation, Episyrphus zerknüllt is exclusively expressed in dorsal stripe, which extends from the anterior pole to about 15\% EL (Figure 16 E, H). This expression domain marks, probably precisely, the anlage of the prospective serosa (Rafiqi et al., in preparation). At the onset of germband extension, the expression follows the spreading of the serosa (Figure 16 F, I, J-L). Unlike Clogmia zerknüllt, Episyrphus zerknüllt is not expressed maternally. However, the zygotic expression of Episyrphus zerknüllt is very similar to zygotic expression of Clogmia zerknüllt, which appears in a slightly broader domain and does not extend quite as far to the posterior pole as Episyrphus zerknüllt at a comparable stage (supplemental Figure S2 A-D; Stauber et al., 2002). Other cyclorrhaphans, such as Drosophila and Megaselia, share with Episyrphus the absence of maternal zerknüllt transcripts in early embryos, but they differ in that their zygotic zerknüllt expression domains do not extend to the anterior pole (supplemental Figure S2 E-H; Doyle et al., 1986; Stauber et al., 1999). Thus, the 
expression of Episyrphus zerknüllt shares similarities with both lower dipterans such as Clogmia, as well as other cyclorrhaphans, and might best be described as an intermediate.

\subsubsection{Episyrphus orthodenticle is not expressed in pre-blastoderm embryos}

Recent studies propose that maternal orthodenticle activity substitutes for bicoid functions in Tribolium and Nasonia (Lynch et al., 2006; Schröder, 2003). A putative orthodenticle homologue was cloned from Episyrphus in an attempt to isolate bicoid. In an alignment of the predicted amino acid sequence with Drosophila orthodenticle/ocelliless (Finkelstein et al., 1990) (Figure 17), the putative Episyrphus homologue shows over $75 \%$ sequence similarity with one of the putative Drosophila orthodenticle/ocelliless protein isoforms, suggesting that the newly identified gene is Episyrphus orthodenticle.

To test whether Episyrphus orthodenticle is expressed maternally, I studied the expression of this gene. In pre-blastoderm embryos, Episyrphus orthodenticle transcripts could not be detected by whole-mount in situ hybridization (data not shown), suggesting that, like in Drosophila, orthodenticle is not maternally expressed in Episyrphus (Finkelstein et al., 1990). However, zygotic Episyrphus orthodenticle transcript is expressed in the anterior $20 \%$ of the embryo (Figure 18 A, B). During cellularization of the blastoderm, these transcripts disappear from the anterior-most blastoderm, and later also from the ventral-most region of the remaining anterior stripe (Figure $18 \mathrm{C}-\mathrm{F}$ ). During cellularization of the blastoderm, orthodenticle expression begins to clear from the dorsal region, resulting in a dorsal stripe free of expression at the onset of gastrulation (Figure 18 D, F). Thus, expression of Episyrphus orthodenticle is reminiscent of the expression of this gene in Drosophila and Anopheles (Finkelstein et al., 1990; Goltsev et al., 2004a). Provided that Episyrphus does not contain additional orthodenticle genes, these data suggest that early zygotic Episyrphus hunchback expression throughout the anterior half of the embryo is under the control of a different gene. 


\section{Discussion}

\subsection{Do all cyclorrhaphan dipterans have bicoid?}

It has been proposed that bicoid evolved in the stem lineage of cyclorrhaphan dipterans (Schmidt-Ott, 2000; Stauber et al., 2002). The identification of bicoid orthologues in Platypeza and Lonchoptera (Figures 2,3) demonstrates that bicoid is in fact widely conserved in basal cyclorrhaphans. In addition, conserved sequence motifs of in the newly isolated bicoid homologues (Figure 3), the analyses of hunchback reporter gene expression in transgenic Drosophila embryos (Figure 12), bicoid RNAi data from Megaselia (Figure 13) (Stauber et al., 2000) and Bicoid binding sites upstream of the Megaselia hunchback P2 promoter (supplemental Figure S3) support the hypothesis of a conserved early patterning role of this gene, not only in higher (Bonneton et al., 1997; Driever and Nüsslein-Volhard, 1989; Driever et al., 1989; Lukowitz et al., 1994; McGregor; McGregor et al., 2001b; Shaw et al., 2001; Struhl et $a l ., 1989)$ but also in lower cyclorrhaphans. However, the PCR-based screen for bicoid homologues in dipterans also suggests that Episyrphus lacks this gene. In Episyrphus, low stringency PCR with degenerate bicoid primers only yielded homeobox genes that are phylogenetically (zerknüllt) or functionally (orthodenticle) related to bicoid (Finkelstein et al., 1990; Lynch et al., 2006; Schröder, 2003; Stauber et al., 1999). This result raises the question how zygotic expression of zygotic hunchback throughout the anterior half of the early Episyrphus embryo (Figure $14 \mathrm{C}$-E) is activated. The question has been approached by the functional comparison of the Episyrphus hunchback enhancer with the hunchback enhancers from other cyclorrhaphans (in which bicoid has been identified) and non-cyclorrhaphan dipterans, respectively. In this comparison, regulatory DNA of Episyrphus hunchback (Figure 12 M, N) differs significantly from regulatory DNA of the other cyclorrhaphan hunchback homologues (Figure $12 \mathrm{G}-\mathrm{L})$. Instead, it shares characteristics with the hunchback regulatory DNA of the noncyclorrhaphan insects Haematopota (Figure 12 Q, P) and Tribolium (Wolff et al., 1998). I cannot exclude the possibility that a Bicoid-response element in Episyrphus is located outside the sequence analyzed (complete intron of the P1 transcript) and was missed. However, all Bicoid-response elements of hunchback genes that have been characterized until now have been mapped to the intron of the P1 transcript (Bonneton et al., 1997; Driever and NüssleinVolhard, 1989; Driever et al., 1989; Lukowitz et al., 1994; Schröder et al., 1988; Shaw et al., 
2001; Struhl et al., 1989; Treier et al., 1989). More importantly, the congruence between the screening data for bicoid orthologues and the transgenic data with regulatory DNAs of hunchback homologues provides independent support for the hypothesis that a bicoid orthologue is absent in Episyrphus.

\subsection{Does Episyrphus reflect the primitive patterning mechanism of cyclorrhaphan flies?}

Episyrphus may have lost bicoid or may primarily lack this gene. Unfortunately, the position of syrphids within Cyclorrhapha is still unclear. Although there is agreement about assigning syrphids to lower cyclorrhaphans (Aschiza; Figure 1), the position within the Aschiza has been subject to controversy (reviewed in Collins and Wiegmann, 2002; reviewed in Yeates and Wiegmann, 1999). In recent studies, taxonomists have favored the hypothesis that Aschiza are paraphyletic and that syrphids, together with a second family (Pipunculidae), constitute the sister-group of Schizophora, which comprises all higher cyclorrhaphans (e.g. Moulton and Wiegmann, 2004, and references therein). This phylogenetic hypothesis is consistent with the fossil record (Grimaldi and Engel, 2005). Currently, the oldest putative syrphid fossil has been described in 80 million year old (myo) amber, while fossils of other basal ashizan taxa (Lonchopteridae, Platypezidae, Phoridae) have been found in 115-130 myo amber (Grimaldi and Engel, 2005). Considering this phylogeny, however, the outcome of my investigations is very unexpected. Not only the loss of an important developmental regulator (bicoid) has to be explained but also the anterior specification of extraembryonic blastoderm (Figure 16 D-L) and the extraembryonic expression of Episyrphus hunchback (Figure 14 J-L and Figure 15), all of which have been reported for non-cyclorrhaphan but not for cyclorrhaphan dipterans (Goltsev et al., 2004a; Rohr et al., 1999; Stauber et al., 2002). Alternatively, syrphids might be an outgroup to the cyclorrhaphans studied. Under this assumption, pattern formation in Episyrphus might reflect the primitive condition in cyclorrhaphans and could be considered as intermediate to lower and higher cyclorrhaphan dipterans. 


\subsection{How did early anterior hunchback regulation change in dipteran evolution?}

Expression of zygotic hunchback in the anterior half of the embryo is conserved throughout the insect order Diptera (Bonneton et al., 1997; Goltsev et al., 2004a; McGregor et al., 2001a; Rohr et al., 1999; Sommer and Tautz, 1991; Stauber et al., 2000; Tautz et al., 1987; Treier et al., 1989). However, the blastoderm fate-map changed in dipteran evolution (Anderson, 1972) and it has been recently pointed out that the extreme expansion of the embryonic blastoderm to the anterior pole in cyclorrhaphans and culicomorphan mosquitoes may have been accompanied by the independent evolution of localized transcripts with a role in head specification (Schmidt-Ott, 2005). In both cyclorrhaphans with bicoid and culicomorphan mosquitoes, where a bicoid-like mRNA has been predicted (see Introduction), the extraembryonic anlage is restricted to dorsal blastoderm and the embryonic primordium extends to the anterior pole, while in other dipterans, the embryonic blastoderm is slightly smaller relative to the egg and the extraembryonic anlage extends to the pole (Anderson, 1972; Sander, 1976). The localization of a head inducing transcription factor to the anterior tip of the developing egg may have caused this shift in the fate map by repressing the extraembryonic anlage at the anterior pole. The high concentration of this transcription factor at the anterior pole may have also gradually shifted the balance between the ancestral activators of anterior hunchback expression in favor of the most abundant activator - Bicoid in case of cyclorrhaphan flies and a protein $\mathrm{X}$ in the case of culicomorphan mosquitoes.

The reverse argument could explain the results for Episyrphus and Clogmia: in both flies, the extraembryonic anlage extends to the anterior pole and in neither species an anterior localized transcript could be isolated. The implication of this argument is that dipterans may use three different modes of early hunchback activation: the bicoid dependent mechanism of most cyclorrhaphans, the ancestral dipteran mechanism, which might involve Nanosdependent posterior repression and an auto-regulatory loop in the anterior, and a third mechanism in culicomorphan mosquitoes involving a localized transcript encoded by gene $X$. 


\section{Summary and Conclusions}

I have shown that the lower cyclorrhaphan fly Episyrphus balteatus (Syrphidae) combines patterning elements of lower and higher flies. Similarities between Episyrphus and lower flies/insects include the expression of zerknüllt at the anterior pole of the blastoderm and the expression of hunchback in the zerknüllt domain (extraembryonic anlage). In addition, I showed that the response of hunchback regulatory DNA of Episyrphus (5.4 kb upstream of the ORF) in transgenic Drosophila is functionally comparable to lower insects rather than higher flies with a bicoid gene. Similarities between Episyrphus and higher flies include the absence of maternal zerknüllt expression, and the expression of hunchback in a posterior stripe of the blastoderm. This mosaic of developmental traits from lower and higher dipterans suggests that early pattern formation in syrphids resembles the ancestral cyclorrhaphan patterning mechanism shortly before the emergence of bicoid.

The correlating occurrence of bicoid and the expansion of the embryonic blastoderm to the anterior pole suggests that both aspects of development are contingent on each other. A cDNA subtraction screen, which was developed during the course of this work, can be used to test this hypothesis in mosquitoes. 


\section{A Appendix}

\section{A.1 Figures and Tables}

\section{A.1.1 Figures}

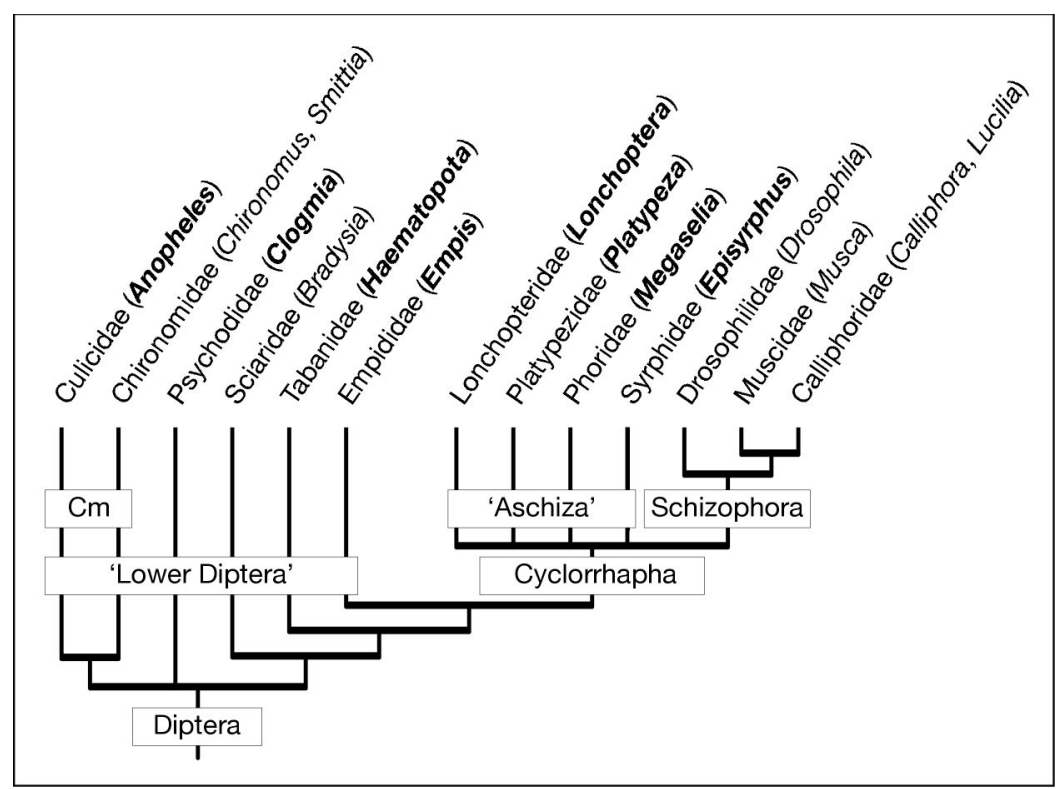

Figure 1. Phylogenetic relationships of taxa mentioned in the text. The insect order Diptera emerged 250 million years ago and comprises about 125,000 to 150,000 species (Grimaldi and Engel, 2005; Yeates and Wiegmann, 1999). The dipterans constitute a strongly supported monophyletic group (Yeates and Wiegmann, 1999). A common character, shared by all dipterans, is the transformation of the second wing pair into halteres (McAlpine, 1989). The Cyclorrhapha emerged about 150 million years ago and comprises about 65,000 species (Grimaldi and Engel, 2005; Yeates and Wiegmann, 1999). Cyclorrhaphans differ from non-cyclorrhaphan or "lower" Diptera in a number of derived characters, which are shared by cyclorrhaphan flies only (e.g. invaginated head capsule of the larva - for a list see McAlpine, 1989). The suborder Cyclorrhapha has been further subdivided into the monophyletic Schizophora and Aschiza, which are probably paraphyletic (Yeates and Wiegmann, 1999). Species that have been analyzed in this work are shown in bold. Abbreviation: Cm, Culicomorpha. Quotes indicate paraphyletic sub-orders. Branch lengths are not to scale. 


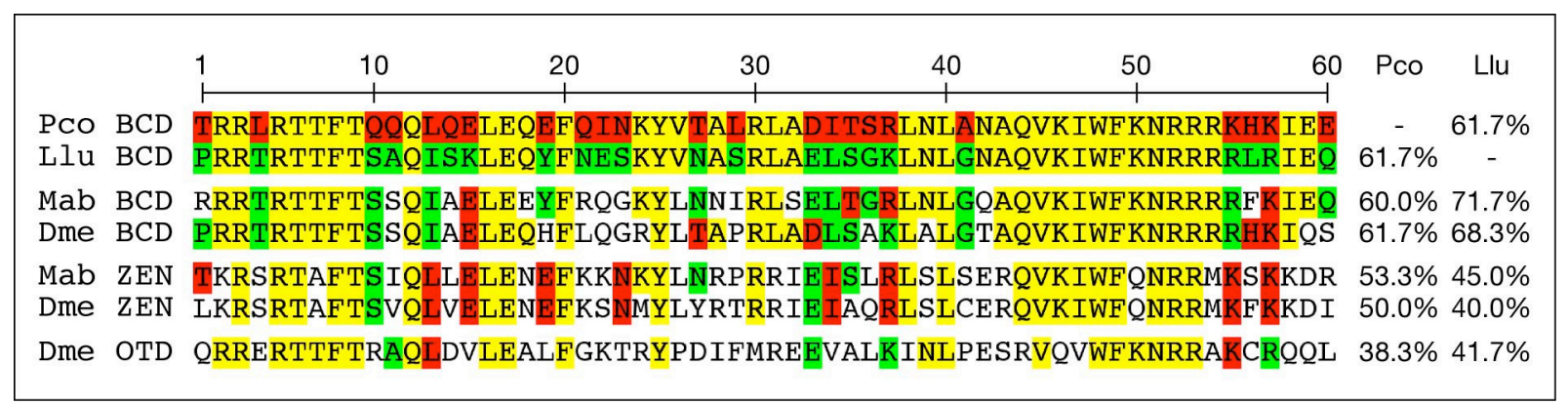

Figure 2. Homeodomain alignment and percent sequence similarity relative to the homeodomains of Platypeza Bicoid and Lonchoptera Bicoid. Abbreviations: Pco BCD, Bicoid of Platypeza (this work); Llu BCD, Bicoid of Lonchoptera (this work); Mab BCD, Bicoid of Megaselia (GenBank entry AJ133024, Stauber et al., 1999); Dme BCD, Bicoid of Drosophila (GenBank entry X07870, Berleth et al., 1988); Mab ZEN, Zerknüllt of Megaselia (GenBank entry AJ133025, Stauber et al., 1999); Dme ZEN, Zerknüllt of Drosophila (GenBank entry X68347, Rushlow et al., 1987); Dme OTD, Orthodenticle of Drosophila (GenBank entry X58983, Finkelstein et al., 1990). Numbers refer to amino acid position, percentage at the right indicate the similarity of the noted homeodomain with the Bicoid homeodomain of Platypeza (Pco) and Lonchoptera (Llu), respectively. Amino acids identical with the homeodomain of Pco BCD are shaded red; amino acids identical with the homeodomain of Llu BCD are shaded green. Amino acids identical with the homeodomains of both, Pco BCD and Llu BCD, are shaded yellow. 


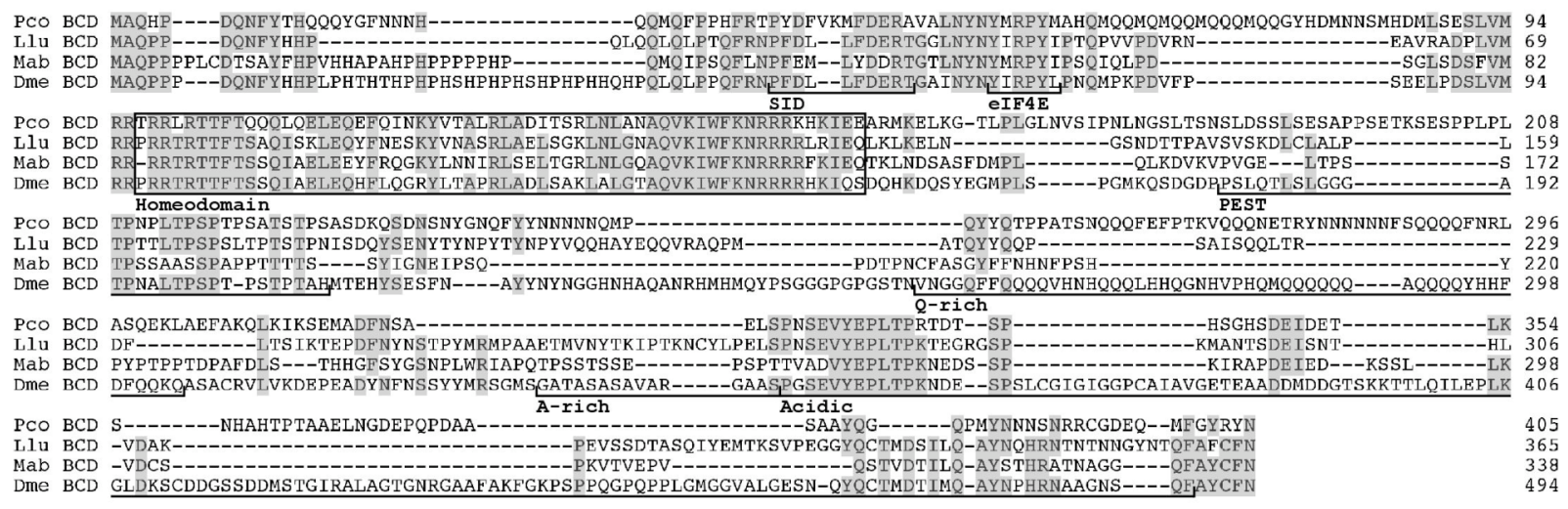

Figure 3. Protein alignment of bicoid homologues. The predicted amino acid sequences of bicoid from Platypeza (Pco BCD), Lonchoptera (Llu BCD), Megaselia (Mab BCD), and Drosophila (Dme BCD) are shown. Amino acids that are conserved in at least 3 sequences (75\%) are shaded in grey; dashes denote gaps. The numbers to the right refer to the last amino acid in each row. The homeodomain is boxed; other conserved domains and motifs of bicoid proteins, which have been functionally characterized (reviewed in McGregor, 2005), are underlined. For GenBank entry numbers of Drosophila and Megaselia Bicoid, see Figure 2. 


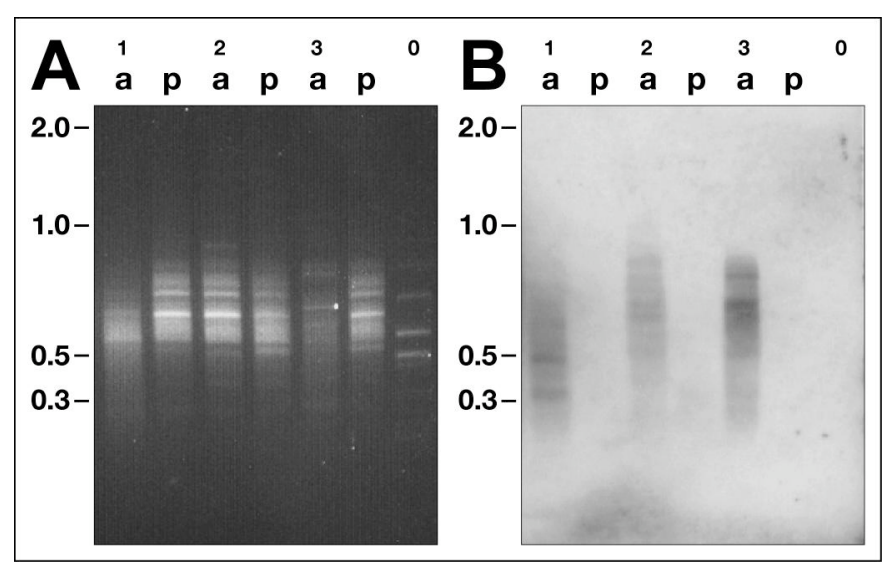

Figure 4. cDNA pools from anterior and posterior cytoplasm of the same Megaselia embryo differ in composition. (A) PCR amplified cDNA from anterior (a) and posterior (p) pole cytoplasm of three Megaselia embryos (1-3); a mock cDNA preparation (0), made in the absence of Megaselia cytoplasm, served as negative control. Single bands in the negative control have possibly been amplified from minute DNA remnants in the enzyme solutions. (B) Southern Blot of the gel shown in (A), hybridized to Megaselia bicoid. Note that only cDNA pools from anterior cytoplasm hybridize with Megaselia bicoid. The smear in these lines is expected due to a truncated reverse transcription reaction during cDNA preparation. As size marker, a $1 \mathrm{~kb}$ DNA Ladder was used (Invitrogen); fragment sizes are given in on the left kilo base pairs. 


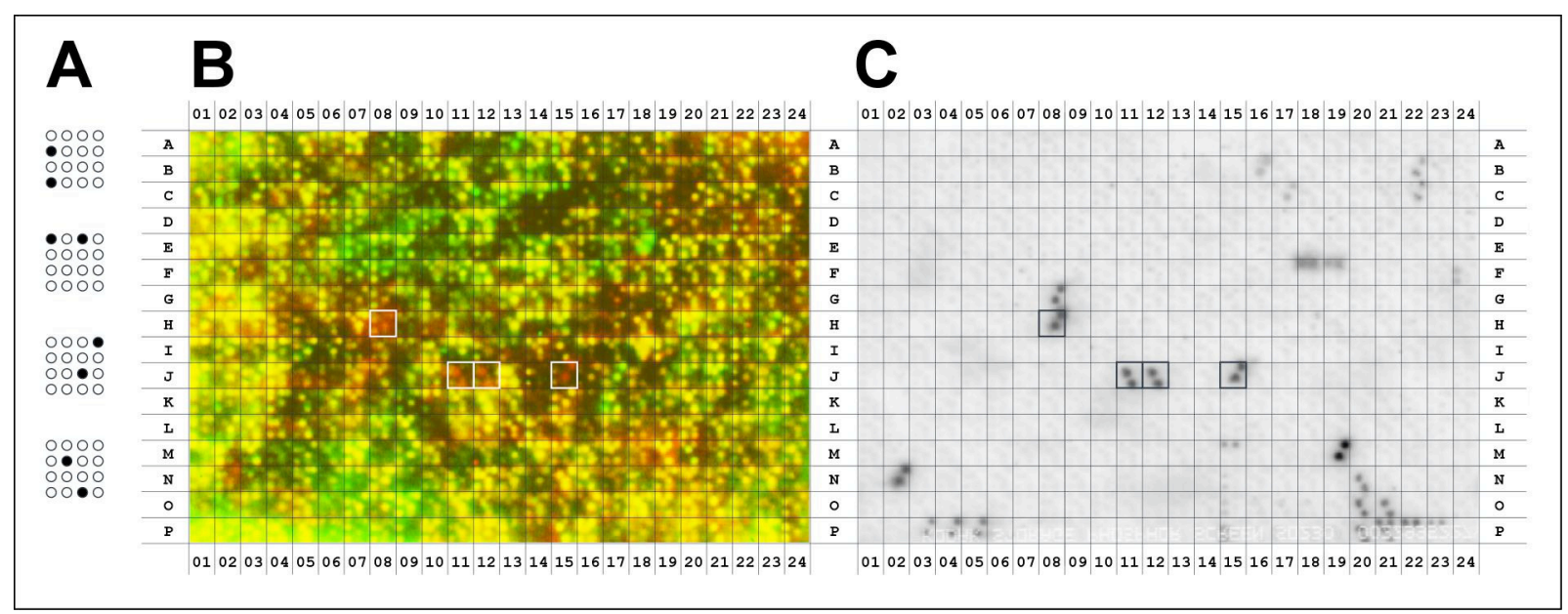

Figure 5. Identification of Megaselia bicoid by subtractive screening of a spotted cDNA library made from anterior egg cytoplasm. (A) 1536 bacterial colonies were spotted onto nylon filters according to a twin-spotting scheme. Within each $3 \mathrm{~mm}$ square (see grid in B and C), four different clones were spotted in the indicated patterns. The remaining eight positions in each square were left blank. (B) Filter replicates of the spotted library were hybridized independently to radioactively labeled cDNA pools prepared from anterior or posterior pole cytoplasm. The signals of the filter replica, which was hybridized with anterior cDNAs, were color-coded in red; the signals of the filter replica, which was hybridized with posterior cDNAs, were color-coded in green. The color-coded images were aligned and merged. Green signals indicate hybridization with the posterior cDNA pool, while red signals indicate hybridization to anterior cDNA only. (C) A third replica of the spotted library was hybridized with a radioactively labeled Megaselia bicoid probe. For four clones (corresponding to the boxed twin spots in B and C), the presence of Megaselia bicoid was verified by sequencing. 


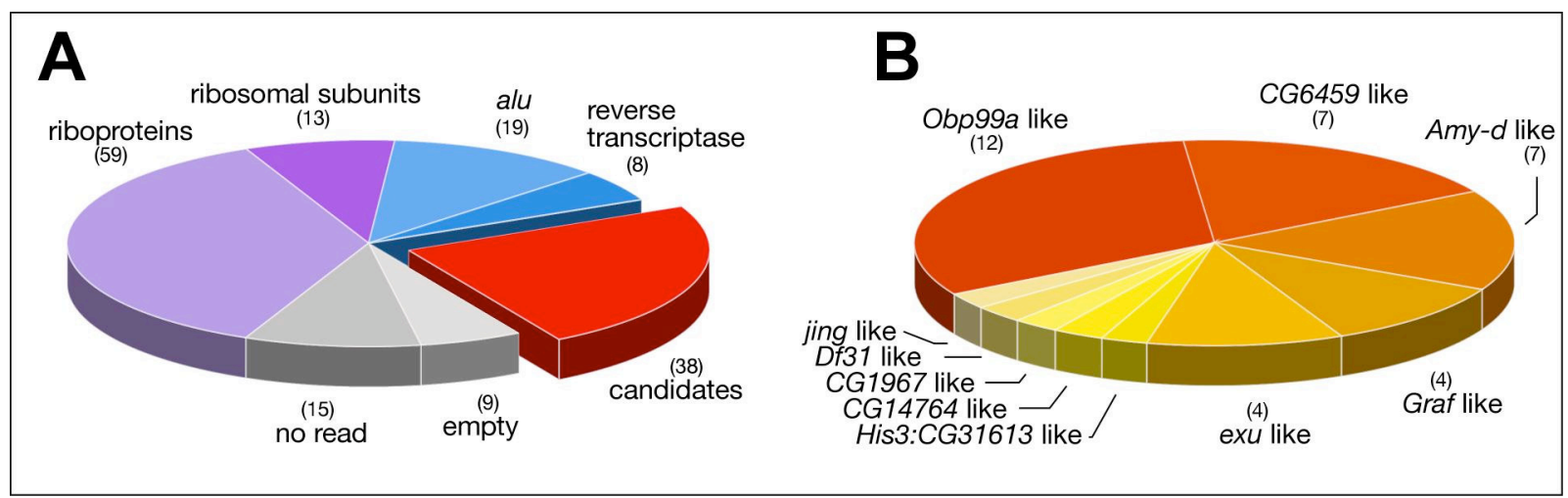

Figure 6. Classification and abundance of Clogmia clones. (A) By subtractive screening, 161 Clogmia clones were isolated from the Clogmia cDNA library of anterior pole cytoplasm. The clones were classified according to their sequence similarity with genes in the GenBank database; the abundance, with which each clone was isolated, is indicated in parentheses. (B) The remaining 38 candidates were compared to Drosophila- and arthropodspecific gene databases, revealing sequences of 10 distinctive transcripts. For details see text and Table 4. 


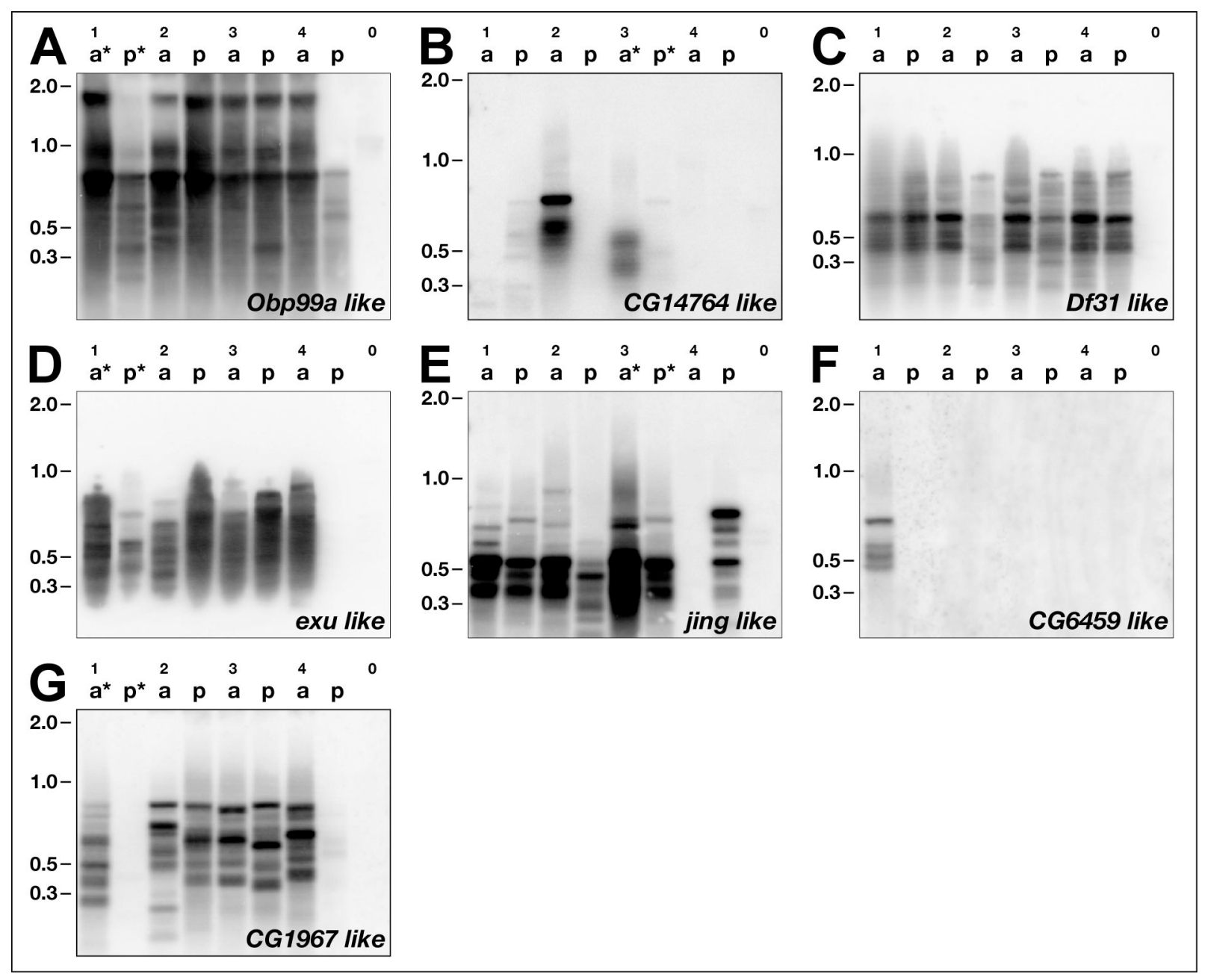

Figure 7. Testing for differential expression of Clogmia candidates in virtual Northern Blots. cDNA was prepared from anterior and posterior pole cytoplasm of Clogmia embryos and amplified by PCR. Of each candidate, a radioactively labeled probe was hybridized to anterior (a) and posterior (p) cDNA of four different $C l o g$ mia embryos (1-4). As negative control, mock cDNA preparations were used, which were prepared in the absence of Clogmia cytoplasm (0). In each panel, asterisks demark those pools of amplified cDNA that have been used to construct and screen the particular anterior cDNA library, from which the tested candidate was isolated. The smear or multiple lanes in the probe hybridizations are expected due to a truncated reverse transcription reaction during cDNA preparation. (A) Obp99a like, hybridized to cDNA of three-hour old embryos. (B) CG14764 like, hybridized to cDNA of one-hour old embryos. (C) Df31 like, hybridized to cDNA of one-hour old embryos. The cDNA pools, which have been used to construct and screen the library from which $D f 31$ like was isolated, have not been included on the blot due to limited cDNA material. (D) exu like, hybridized to cDNA of three-hour old embryos. (E) jing like, hybridized to cDNA of one-hour old embryos. (F) CG6459 like, hybridized to cDNA of one-hour old embryos. The cDNA pools, which have been used to construct and screen the library from which CG6459 like was isolated, have not been included on the blot due to limited cDNA material. (G) CG1967 like, hybridized to cDNA of three-hour old embryos. The same cDNA preparations have been used for the blots in panels A, D, and G, in panels B and E, and in panels C and F, respectively. Size markers on the left of each panel are given in kilo base pairs. For details, see text. 


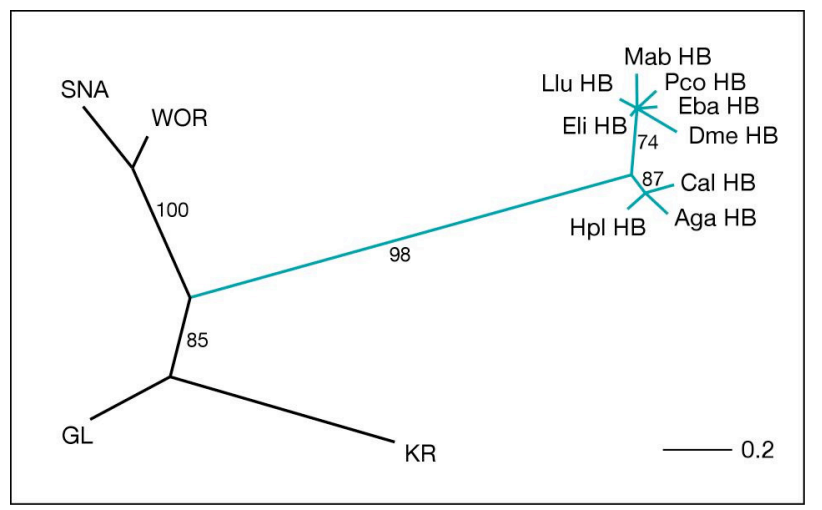

Figure 8. Quartet Puzzling analysis of the newly identified Hunchback homologues. 108 amino acids comprising the conserved $\mathrm{N}$-terminal zinc finger domain (corresponding to amino acids 242-349 of the Drosophila protein) of the predicted Hunchback protein sequences were compared with the zinc finger domains of the four most closely related hunchback paralogs of Drosophila using the Quartet Maximum-Likelihood Method of Strimmer and von Haeseler (Strimmer and von Haeseler, 1996). Numbers refer to reliability values of the branching pattern in percent; branch lengths indicate the average number of amino acid changes per position (see scale). Abbreviations are Dme HB, Hunchback of Drosophila (GenBank entry Y00274, Tautz et al., 1987); Eba HB, Hunchback of Episyrphus (this work); Mab HB, Hunchback of Megaselia (GenBank entry AJ295635, Stauber et al., 2000); Pco HB, Hunchback of Platypeza (this work); Llu HB, Hunchback of Lonchoptera (this work); Eli HB, Hunchback of Empis (this work); Hpl HB, Hunchback of Haematopota (this work); Cal HB, Hunchback of Clogmia (this work); Aga HB, Hunchback of Anopheles (Zdobnov et al., 2002); and of the Hunchback paralogues in Drosophila, GL, Glass (GenBank entry X15400, Moses et al., 1989); KR, Krüppel (GenBank entry X03414, Rosenberg et al., 1986); SNA, Snail (GenBank entry Y00288, Boulay et al., 1987); WOR, Worniu (GenBank entry AF118857, Ashraf et al., 1999). 


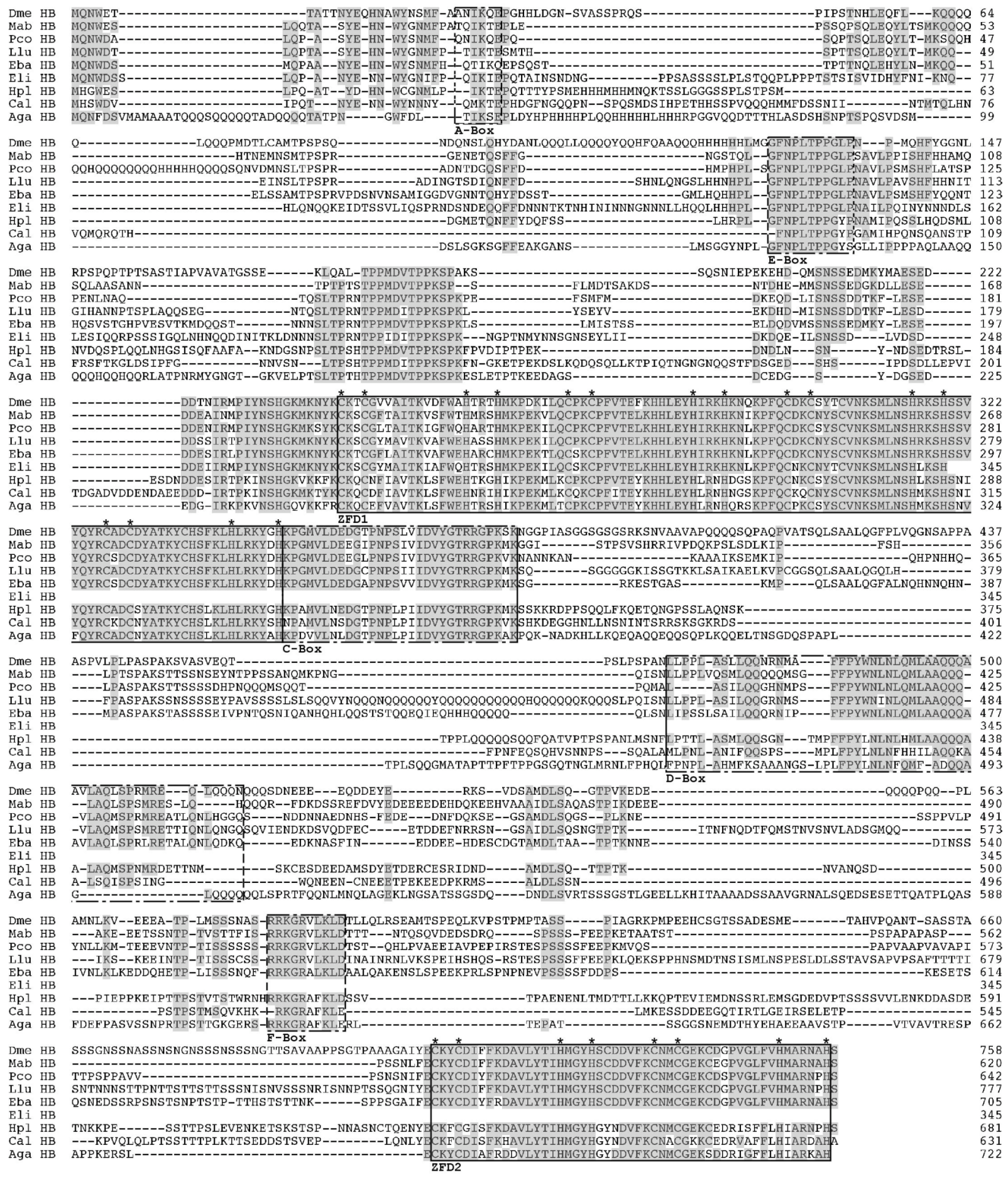

Figure 9. Alignment of predicted protein sequences of the newly identified hunchback homologues. Amino acids conserved in at least 5 sequences (56\%) are shaded in grey; dashes denote gaps. Asterisks mark the relevant cysteine and histidine residues of the zinc fingers (Tautz et al., 1987). The numbers to the right refer to the last amino acid in each row. The highest similarity is seen in the zinc finger domains ZFD1 and ZFD2 (boxed). Outside of the zinc finger domains, the sequence is conserved in previously defined regions (dashed boxes) (Hülskamp et al., 1994; McGregor et al., 2001a; Tautz et al., 1987), suggesting that these regions are of structural or functional importance. Abbreviations as in Figure 9. The predicted protein sequences are based on cDNA sequences, for Empis, only the N-terminal portion of the predicted protein sequences is known. 


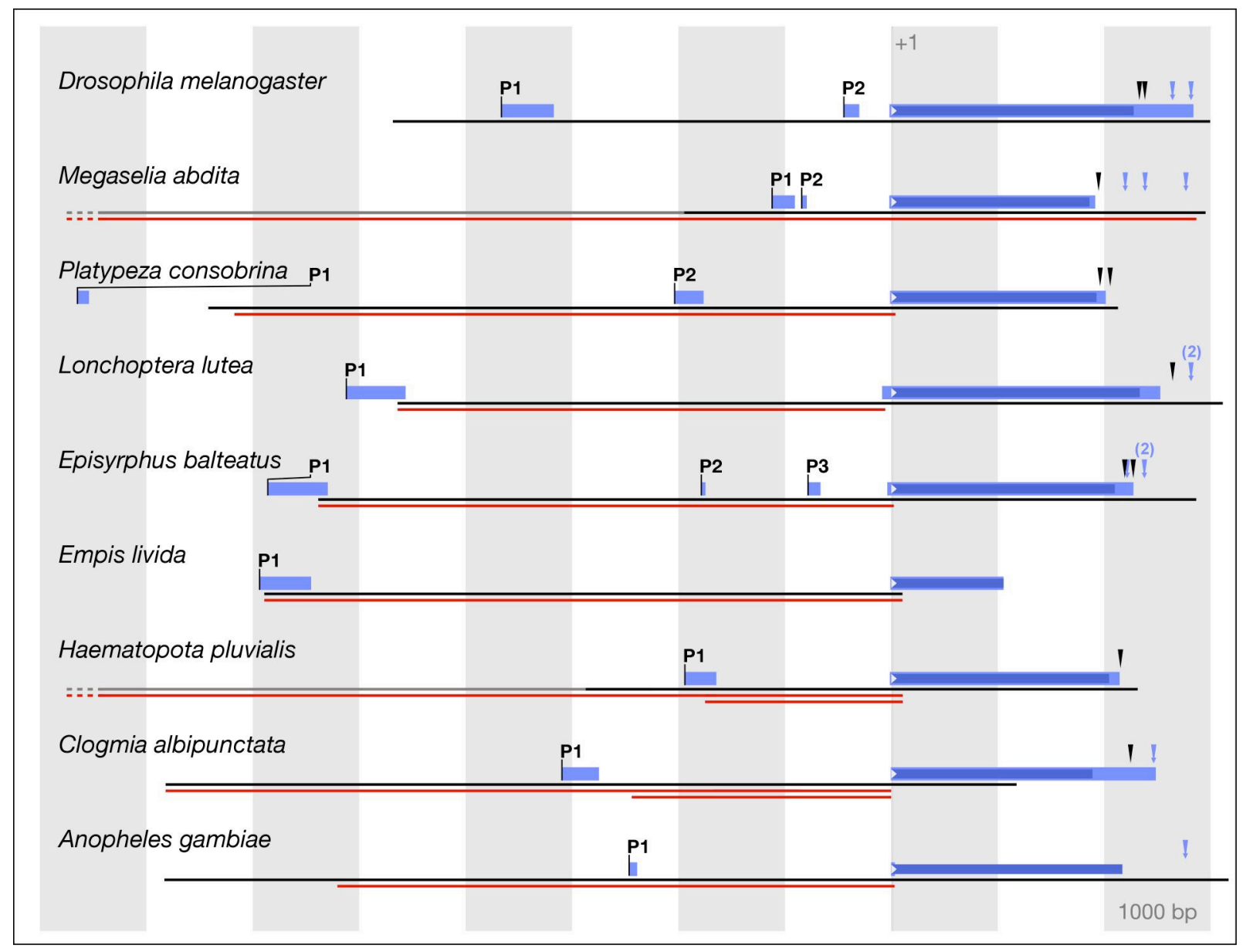

Figure 10. Genomic organization of Dipteran hunchback homologues. For each species, hunchback cDNA (light-blue: untranslated region, dark-blue: ORF) has been mapped to genomic DNA (black: sequenced, grey: not sequenced). All sequences were aligned relative to nucleotide position +1 as the start of the ORF; one vertical bar equates to $1 \mathrm{~kb}$ of sequence. Putative promoters are indicated as vertical lines in front of the leading exons. The stretch of genomic DNA, which has been analyzed in transgenic Drosophila embryos, is indicated below the genomic organization (red). Black wedges indicate putative NRE sequences (Wharton and Struhl, 1991), for an alignment of the putative NREs see Figure 11; blue arrows denote the sequence AATAAA as putative polyadenylation signal (reviewed in Birnstiel et al., 1985). The P1 exon of Platypeza is not positioned in scale. The dotted lines for Megaselia and Haematopota indicate additional upstream regulatory sequence. All sequences have been documented in the Appendix A.3. For details, see text. 


\begin{tabular}{|c|c|c|}
\hline & & .T.GTTGTC.A. .ATTGTA.ATA \\
\hline Mdo & 1 & TTTTTTTCGTTGСTTTGAАTTGTAААTAАTTA \\
\hline Mdo & 2 & AGTGAATCGTTGTCATGAATTGTAAATATGAA \\
\hline Dme & 1 & АTATAATCGTTGTCCAGAATTGTATATATTCG \\
\hline Dme & 2 & АTTATTTTGTTGTCGAAAATTGTACATAAGCC \\
\hline Dvi & 1 & САTATTTCGTTGTCCAGAATTGTAAATACTCG \\
\hline Dvi & 2 & TTGATTTTGTTGTCGAGAATTGTACATAAGCC \\
\hline $\mathrm{Mab}$ & & CAAAAACTGTTGTCAAAGATTGTACATATGAA \\
\hline $\mathrm{PCO}$ & 1 & TATTATTTGTTGTCAAAGATTGTACATATGAA \\
\hline $\mathrm{PCO}$ & 2 & TAAGAAAAGTTGTCAAGGATTGTACATAAAAA \\
\hline Llu & & AGCACAATGTTGTC-ATAATTGTACATAAAAA \\
\hline Eba & 1 & AGAGTTTCGTTGTCAAAGATTGTAAATATTAA \\
\hline Eba & 2 & АAАATACTGTTGTCCAAАATTGTACATACTAT \\
\hline Hpl & & AGCGCTTTGTTGTGTAGAATTCAACTTGAAAT \\
\hline Cal & & АTTTGATCGTTGTATA-GATTGTTGTTATATT \\
\hline
\end{tabular}

Figure 11. Putative nanos response element (NRE) sequences from dipteran hunchback homologues. Nucleotides shared by at least ten sequences $(71 \%)$ are shaded in grey and given as consensus. In addition to the NRE sequences in hunchback of Drosophila melanogaster (Dme), putative NRE sequences in dipteran hunchback homologues have been previously identified in Drosophila virilis (Dvi) (GenBank entry X15359, Hancock et al., 1999; GenBank entry X15359, Treier et al., 1989) and Musca domestica (Mdo) (GenBank entry Y13050, Bonneton et al., 1997). During the course of this study, putative NRE sequences have been identified in hunchback homologues of Megaselia, Platypeza, Lonchoptera, Episyrphus, Haematopota, and Clogmia. For the positions of the NRE sequences within the respective hunchback loci, see Figure 8 and in the sequences noted in the Appendix A.3. For further abbreviations, see legend of Figure 9. 

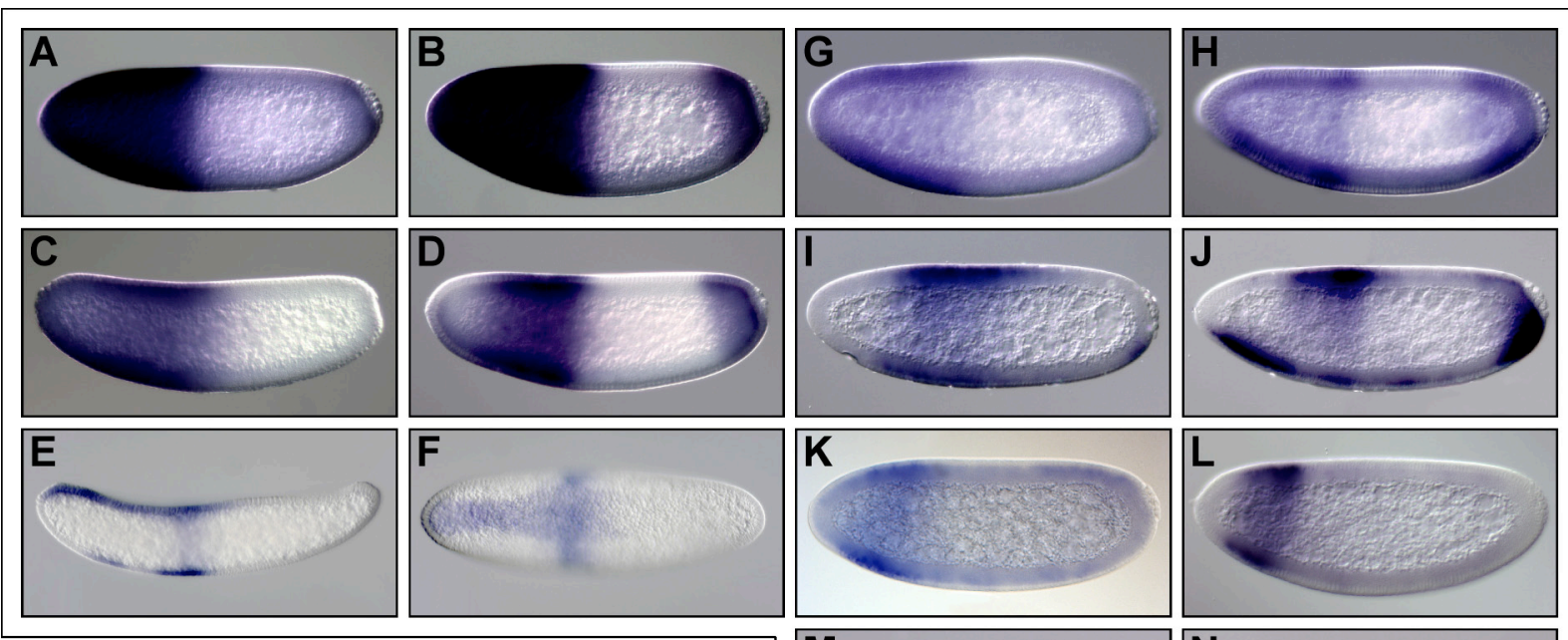

Figure 12. Functional analysis of hunchback regulatory DNA by reporter gene expression in Drosophila embryos. For each dipteran species, hunchback regulatory DNA upstream of the ORF was cloned in front of a reporter gene and integrated into the genome of Drosophila. Unless mentioned otherwise, expression of the reporter gene was assayed by whole-mount in situ hybridization at two time points: onset of cellularization (respective left panel), and mid-cellularization (respective right panel). Embryos are shown in lateral view, unless indicated otherwise; anterior is to the left. (A, B) Drosophila, endogenous hunchback expression. (C, D) Megaselia, endogenous hunchback expression.
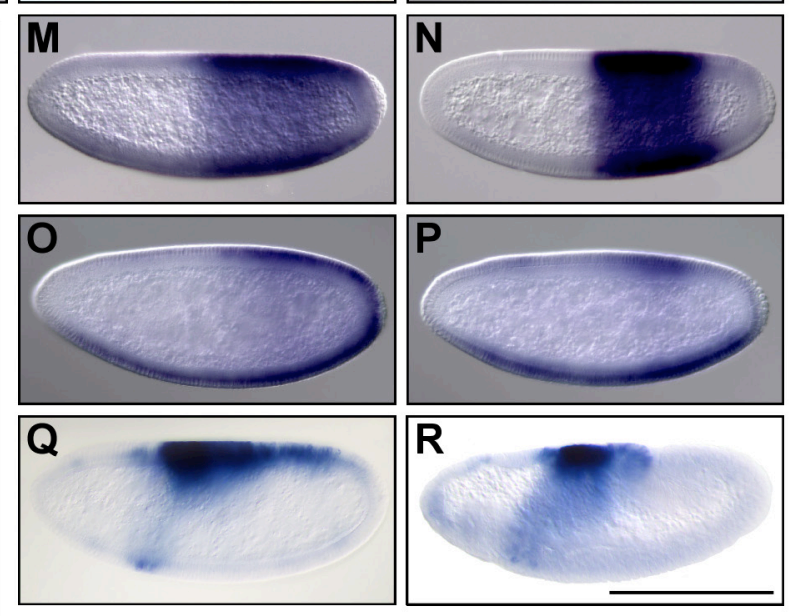

(E, F) Clogmia, endogenous hunchback expression during late cellularization, lateral (E) and dorsal (F) view, respectively. (G, H) Expression driven by $10 \mathrm{~kb}$ of the Megaselia hunchback locus. (I, J) Expression driven by $6.2 \mathrm{~kb}$ of Platypeza hunchback regulatory DNA. (K, L) Expression driven by $4.6 \mathrm{~kb}$ of Lonchoptera hunchback regulatory DNA. (M, N) Expression driven by $5.4 \mathrm{~kb}$ of Episyrphus hunchback regulatory DNA. (O, P) Expression driven by $9 \mathrm{~kb}$ of Haematopota hunchback regulatory DNA. (Q) Expression driven by $6.9 \mathrm{~kb}$ of Clogmia hunchback regulatory DNA (K2) during cellularization and (R) at the onset of gastrulation. Expression driven by a $2.4 \mathrm{~kb}$ sub-fragment of the $6.9 \mathrm{~kb}$ fragment (K13) shows the same pattern (data not shown). lacZ was used as reporter gene in all constructs, except for Megaselia, where Megaselia hunchback was used. For positions of the tested fragments within the respective hunchback loci, see Figure 10 and Table 5. Scale bar: $215 \mu \mathrm{m}$ in A, B, G$\mathrm{R} ; 225 \mu \mathrm{m}$ in C, D; $180 \mu \mathrm{m}$ in E, F. Panels Q, R: courtesy of Alexander Prell. 

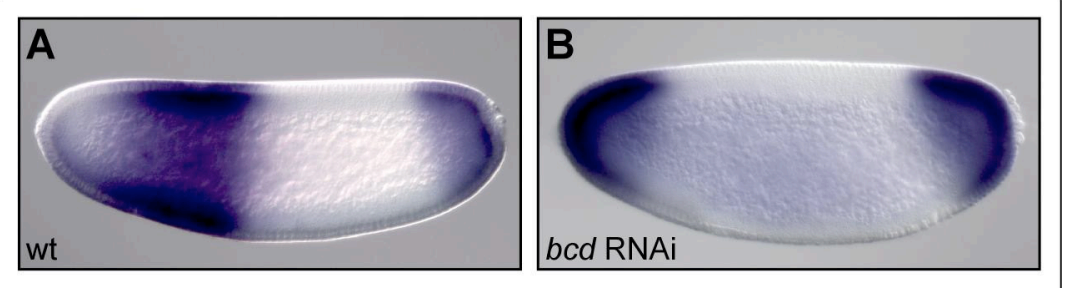

Figure 13. Effects of bicoid RNAi on hunchback expression in Megaselia. Whole-mount in situ hybridizations of Megaselia hunchback. bicoid RNAi was performed as previously described (Stauber et al., 2000), embryos are shown during cellularization.

(A) In the wild-type embryo hunchback expression has started to clear from the anterior pole. (B) A strong bicoid RNAi phenotype is shown. The posterior hunchback expression is duplicated at the anterior pole. Anterior is to the left, dorsal is up. Suppression of anterior clearance at the onset of cellularization and reduction of the anterior expression domain was observed in $68 \%$ of the RNAi embryos $(n=56)$. Buffer injected embryos $(n=16)$ did not show a phenotype. 

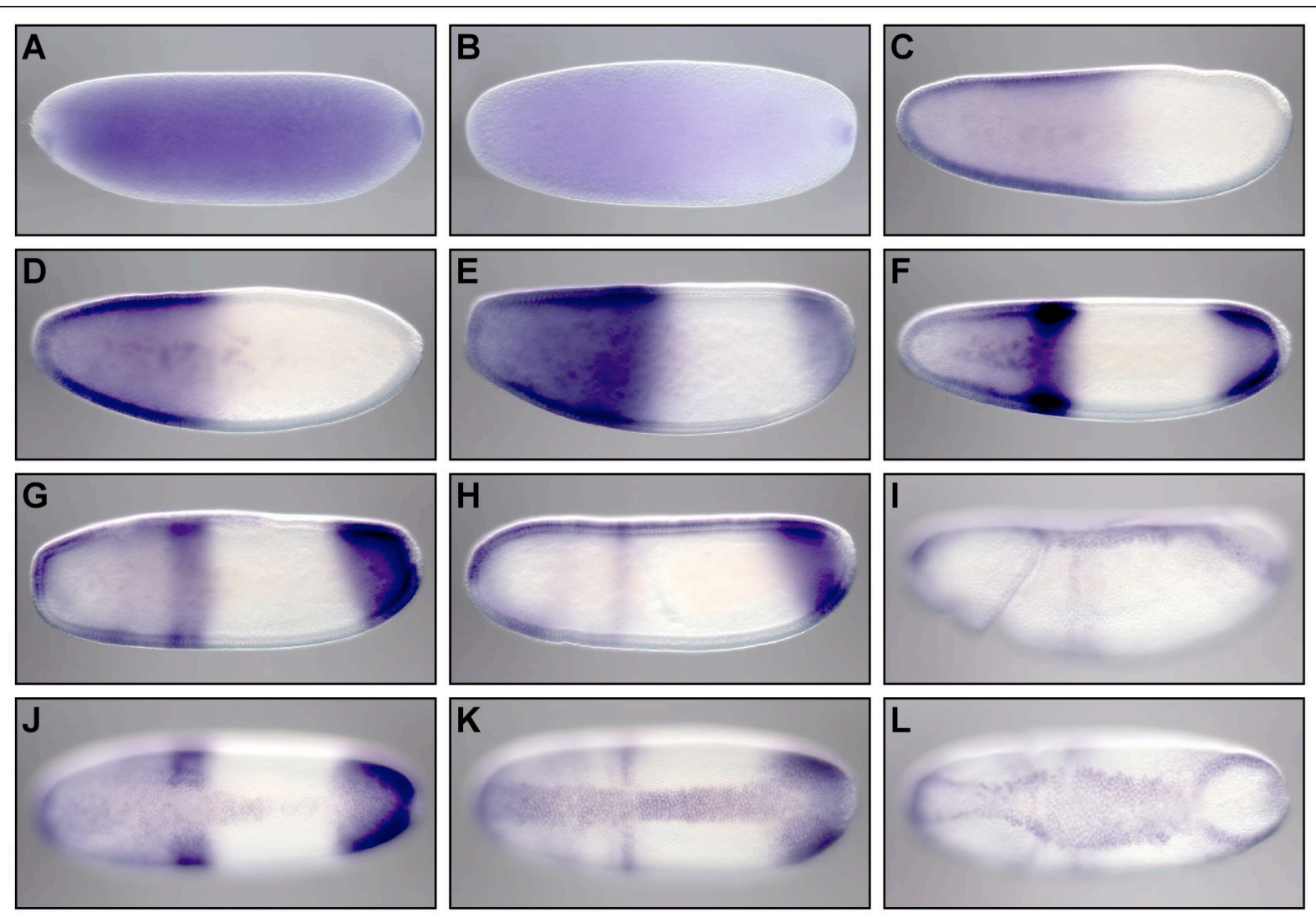

Figure 14. Expression of Episyrphus hunchback. Whole-mount in situ hybridizations showing embryos at (A, B) pre-blastoderm, (C-F) syncytial blastoderm, (G) cellular blastoderm, (H) the onset of gastrulation, and (I) the beginning of germband extension. Embryos are shown in lateral view. (J-L) Dorsal views of the same embryos shown in $(\mathrm{G}),(\mathrm{H})$ and $(\mathrm{I})$, respectively. Anterior is to the left. See text for details. 

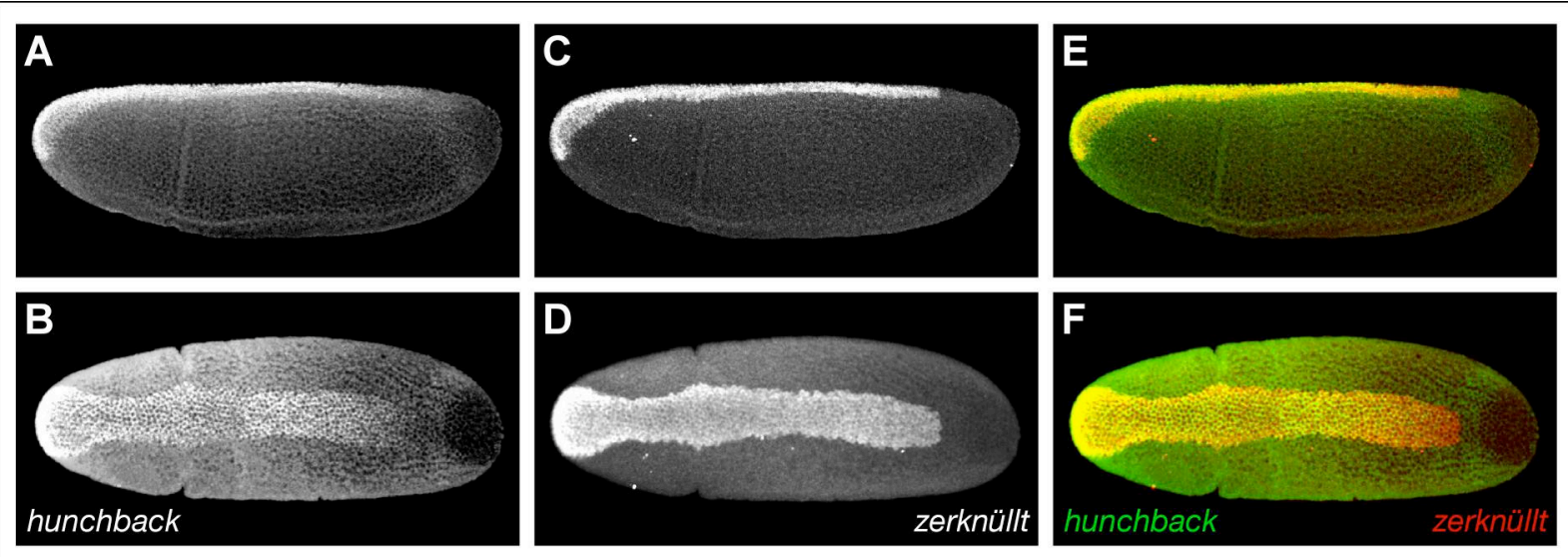

Figure 15. In Episyrphus, hunchback and zerknüllt are co-expressed along the dorsal midline. hunchback and zerknïllt expression in Episyrphus embryos at the onset of gastrulation were analyzed in a single embryo by fluorescent whole-mount in situ hybridization. (A, B) Lateral and dorsal view of Episyrphus hunchback expression. (C, D) Lateral and dorsal view of Episyrphus zerknüllt expression. (E) Merged image of A and C, (F) merged image of $\mathrm{B}$ and D. hunchback expression was false-colored in red; zerknüllt expression was false-colored in green; embryos are oriented with anterior to the left. 

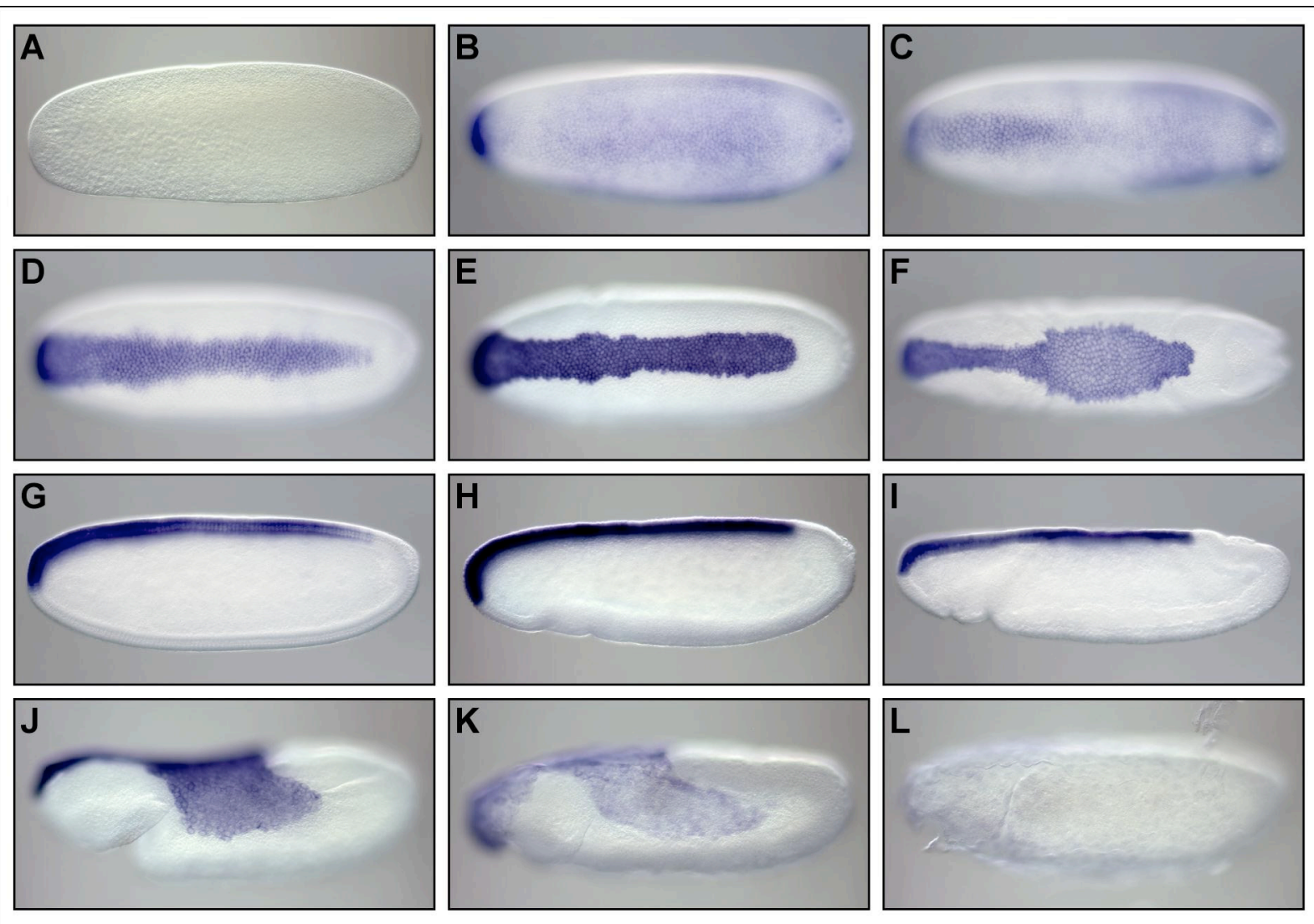

Figure 16. Expression of Episyrphus zerknüllt. Whole-mount in situ hybridizations showing embryos at (A) pre-blastoderm, (B-D) syncytial blastoderm, (E) the onset of gastrulation, (F) the beginning of germband extension, and (J-L) during germband extension. Embryos are shown in lateral orientation. (G-I) Dorsal views of the same embryos shown in (D), (E) and (F), respectively. Anterior is to the left. 


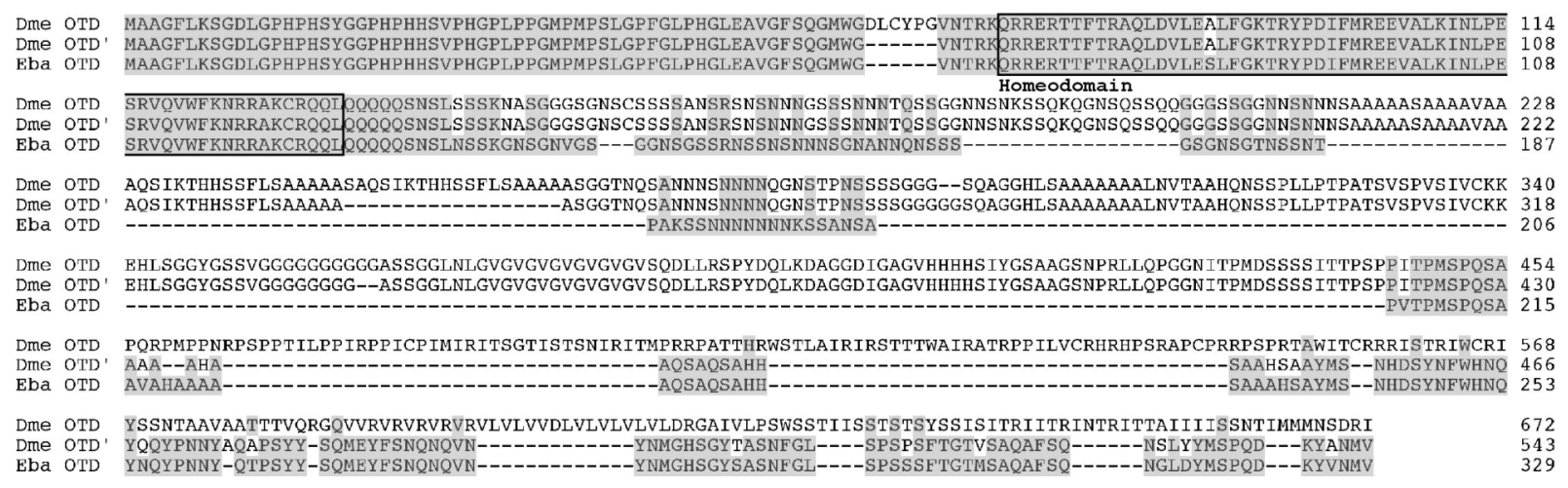

Figure 17. Protein alignment of Episyrphus Orthodenticle with two isoforms of Drosophila Orthodenticle/Ocelliless. The predicted amino acid sequences of orthodenticle from Episyrphus (Eba OTD), and two Drosophila orthodenticlelocelliless transcripts are shown (Dme OTD, GenBank entry X58983; Dme OTD', Genbank entry BT011185). Amino acids that are identical with Eba OTD are shaded in grey; dashes denote gaps. The numbers to the right refer to the last amino acid in each row. The homeodomain is boxed. 

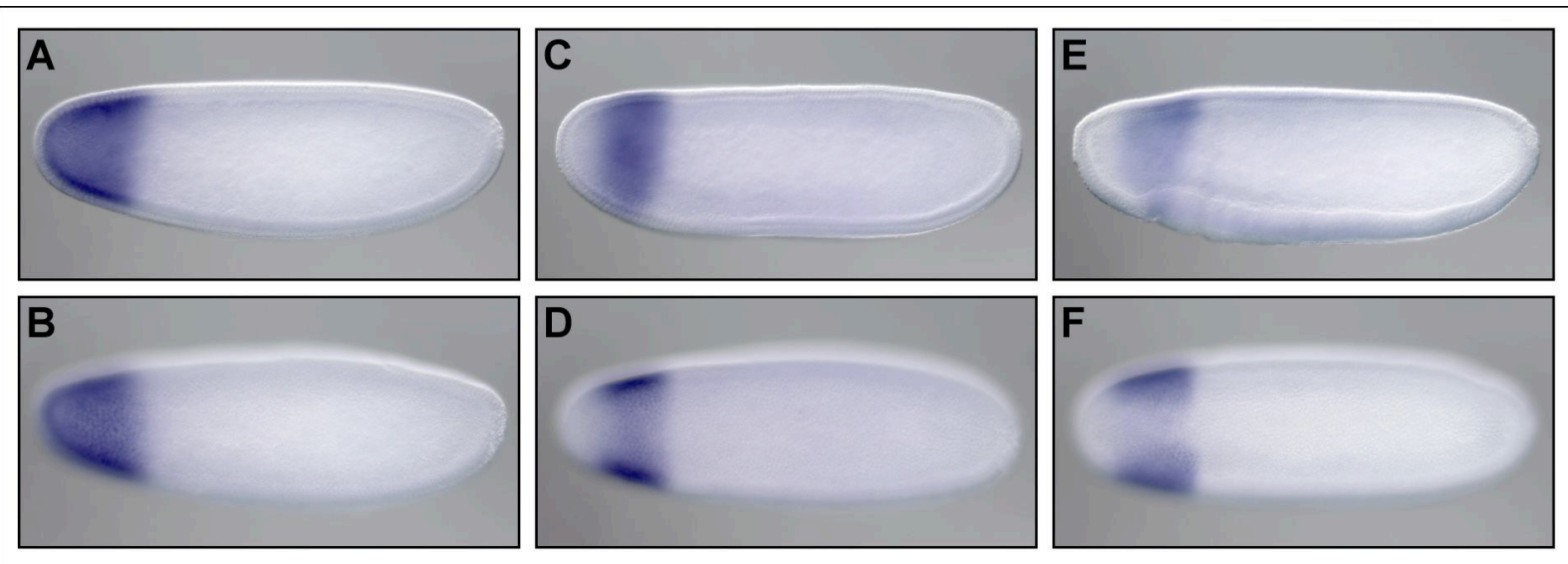

Figure 18. Expression of Episyrphus orthodenticle. Whole-mount in situ hybridizations showing embryos (A, B) at the beginning of blastoderm cellularization, (C, D) at mid-cellularization, and (E, F) at the onset of gastrulation. Embryos are shown in lateral (A, C, E) and in dorsal view (B, D, F). Anterior is to the left. For details, see text. 


\section{A.1.2 Tables}

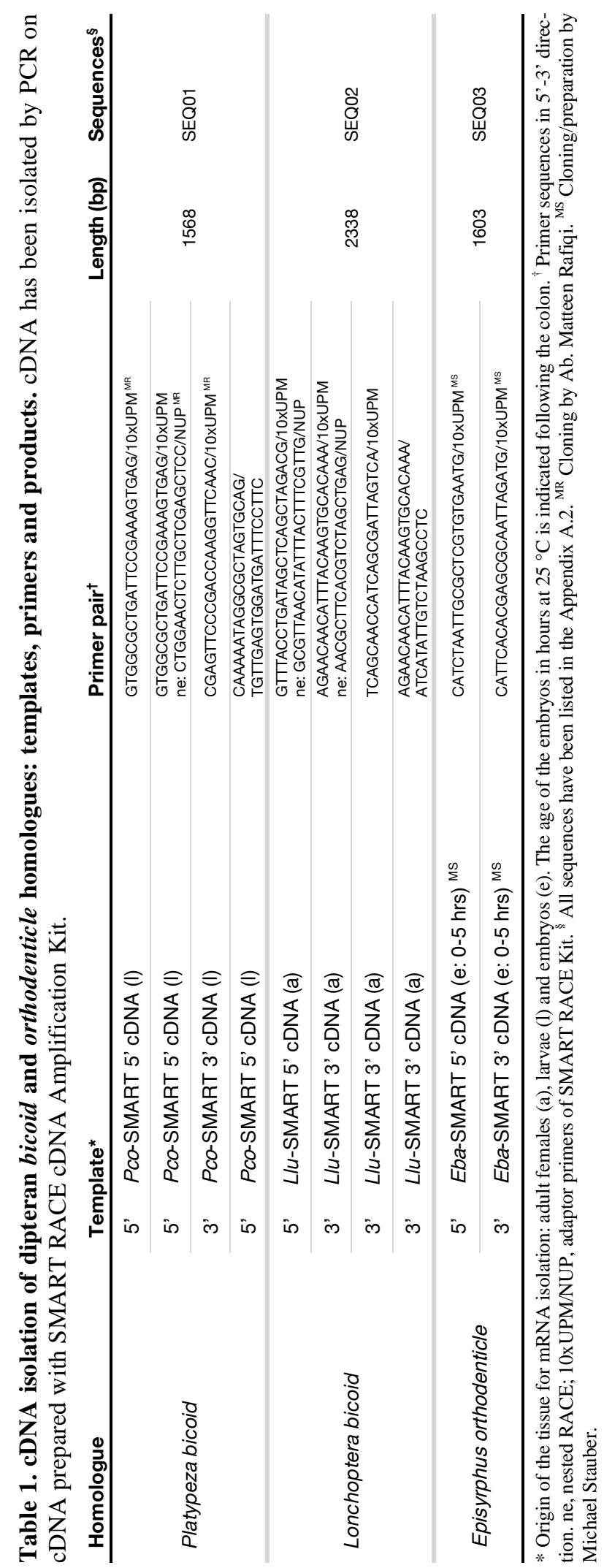




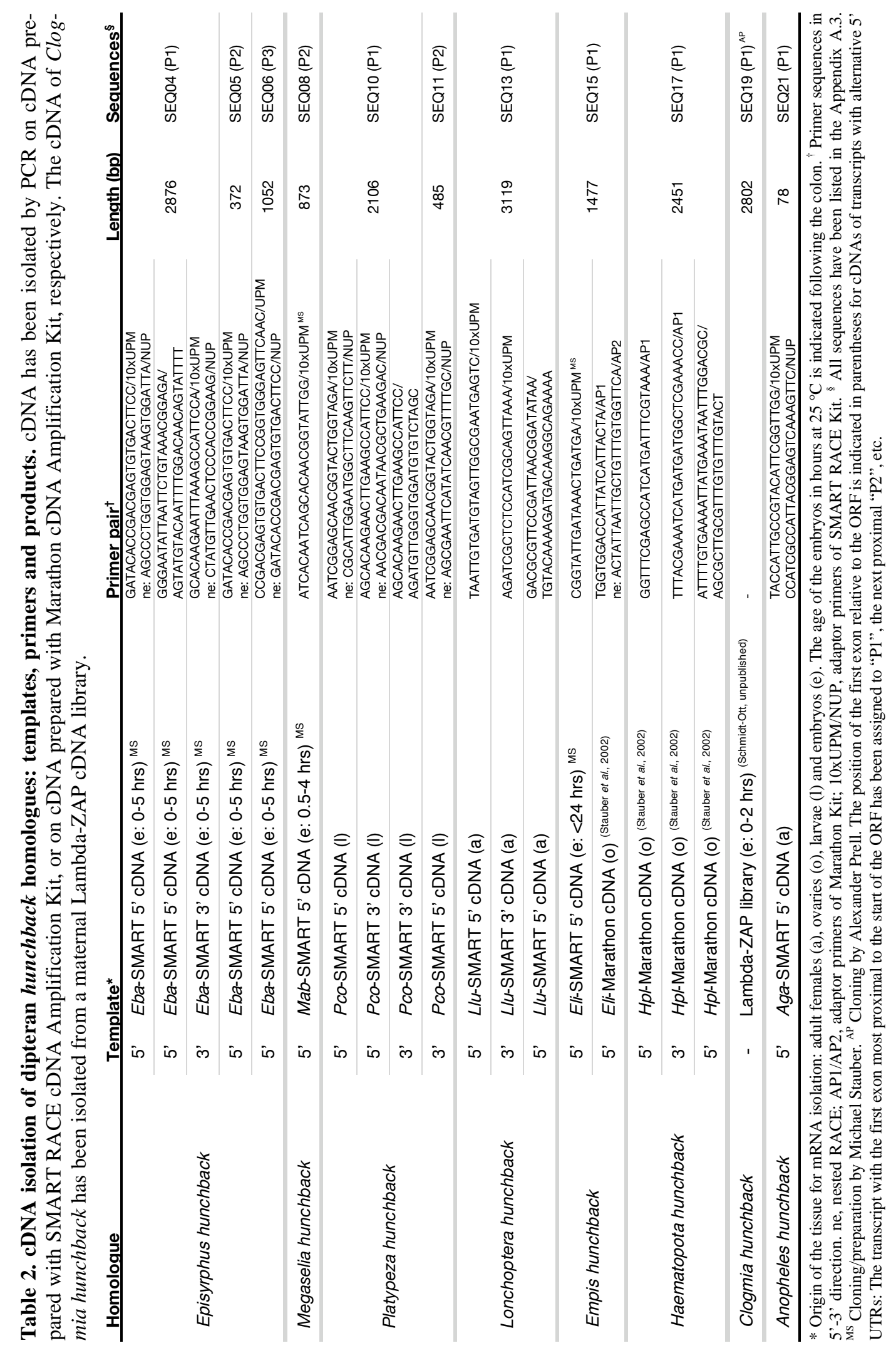


Table 3. RNA probes for whole mount in situ hybridization.

Antisense RNA probe

hunchback

Episyrphus hunchback

Megaselia hunchback

Clogmia hunchback

zerknüllt

Episyrphus zerknüllt

Megaselia zerknüllt

Episyrphus orthodenticle

lacZ

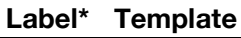

DIG $2.4 \mathrm{~kb}$ genomic Xbal fragment comprising the region of -14 to +2422 relative to the first nucleotide of the ORF (Tautz et al., 1987).

DIG/ $1.1 \mathrm{~kb}$ P3 5' RACE product, comprising $163 \mathrm{bp}$ of UTR and the adjacent nucleotides FITC 1 to 889 of the ORF (this work)

DIG $1.1 \mathrm{~kb}$ 3' RACE product, comprising nucleotides 797 to 1863 of the ORF and $53 \mathrm{bp}$ of the adjacent UTR (Stauber et al., 2002).

FITC $2.1 \mathrm{~kb}$ cDNA fragment, comprising nucleotides 433 to 1896 of the ORF and $593 \mathrm{bp}$ of the adjacent 3' UTR (Rohr et al., 1999).

DIG $\quad 1.4$ kb cDNA (ps60-7, gift from Siegfried Roth).

DIG/ $1 \mathrm{~kb}$ 3' RACE product, comprising nucleotides 120 to 993 of the ORF and $56 \mathrm{bp}$ of the BIO adjacent UTR (Rafiqi et al., in preparation).

DIG $\quad 0.8 \mathrm{~kb}$, complete ORF (Stauber et al., 1999).

BIO $0.9 \mathrm{~kb}$ 3' RACE product, comprising nucleotides 219 to 769 of the ORF and $94 \mathrm{bp}$ of the adjacent UTR (this work).

DIG lacZ ORF (pBST-lacZ, gift from Ronald Kühnlein)

* Independently prepared probes with differently conjugated UTP analogues are not listed separately. Instead, both label types are listed, separated by a slash. Abbreviation: BIO, biotin; DIG, digoxigenin; FITC, fluorescein. 
Table 4. Sequence comparison of Clogmia candidates with Drosophila and Anopheles genes. The putative functions of the proteins encoded by ten distinct Clogmia cDNAs were assessed by sequence comparison to Anopheles and Drosophila genes. Clogmia cDNAs were named after the putatively closest related sequence in Drosophila. The Expect value describes for a given query sequence, how often an equally good or better alignment could have been found in the database by chance (Altschul et al., 1994). The Expect value is often written as $\mathrm{x}$ to the power of $e$; here it is converted to $\mathrm{x}$ to the power of 10 as a more comprehendible tool to assess homology. Expect values lower than 0.01 were considered as reasonable support for homology, whereas Expect values higher than 1 were not considered as support for homology. For all Clogmia candidates, the identified Anopheles sequences were themselves homologues to the Drosophila genes. The degree of conservation serves as a visualization of the Expect value, while the abundance indicates, how often a particular cDNA was isolated among the 161 sequenced clones. The origin from one-hour and three-hour old embryos, respectively, is indicated in parentheses.

\begin{tabular}{|c|c|c|c|c|c|c|}
\hline cDNA & Putative molecular function & $\begin{array}{l}\text { Expect value } \\
\text { (Anopheles) }\end{array}$ & $\begin{array}{l}\text { Expect value } \\
\text { (Drosophila) }\end{array}$ & $\begin{array}{l}\text { Degree of } \\
\text { conservation }\end{array}$ & \multicolumn{2}{|c|}{$\begin{array}{l}\text { Abundance } \\
\text { in screen }\end{array}$} \\
\hline \multicolumn{7}{|c|}{ Chosen for analyses of transcript localization, based on putative molecular function and high degree of conservation } \\
\hline Obp99a like & pheromone binding & $2 \cdot 10^{-13}$ & $5 \cdot 10^{-10}$ & +++ & 12 & $(5 / 7)$ \\
\hline exu like & RNA localization & $6 \cdot 10^{-6}$ & $2 \cdot 10^{-3}$ & ++ & 4 & $(-/ 4)$ \\
\hline Df31 like & chromatin remodeling & $5 \cdot 10^{-2}$ & $7 \cdot 10^{-3}$ & ++ & 1 & $(1 /-)$ \\
\hline CG14764 like & unknown & $5 \cdot 10^{-12}$ & $7 \cdot 10^{-10}$ & +++ & 1 & $(1 /-)$ \\
\hline CG1967 like & post-Golgi transport & $2 \cdot 10^{-40}$ & $3 \cdot 10^{-35}$ & +++ & 1 & $(1 /-)$ \\
\hline CG6459 like & mitochondrial & $7 \cdot 10^{-3}$ & 2.8 & + & 7 & $(7 /-)$ \\
\hline jing like & transcription factor & 1.7 & 4.5 & - & 1 & $(1 /-)$ \\
\hline \multicolumn{7}{|c|}{ Putative house-keeping functions and/or lack of conservation in lower dipterans and other insects } \\
\hline His3:CG31613 like & histone & $2 \cdot 10^{-32}$ & $1 \cdot 10^{-31}$ & +++ & 1 & $(-/ 1)$ \\
\hline Graf like & Rho GTPase & no hit & 4.9 & - & 4 & $(3 / 1)$ \\
\hline Amy-d like & sugar metabolism & no hit & 4.9 & - & 6 & $(6 /-)$ \\
\hline
\end{tabular}


Table 5. Reporter gene constructs to analyze hunchback regulatory DNA in transgenic Drosophila embryos. Putative regulatory DNA of the newly isolated hunchback homologues was cloned in front of a reporter gene and integrated by P-element mediated germline transformation into the genome of Drosophila. Except for Platypeza, the DNA fragments cloned from each species included the intron of the respective P1 transcript. For Haematopota and Clogmia, two reporter gene constructs were analyzed. The positions of the P1 leader, the P1 intron and the region tested in transgenic Drosophila embryos are given relative to the start of the ORFs $(+1)$. The number of established transgenic lines and the number of lines that showed the same reporter expression (in parentheses) are listed.

\begin{tabular}{|c|c|c|c|c|c|c|}
\hline \multirow{2}{*}{$\begin{array}{l}\text { Species } \\
\text { Episyrphus }\end{array}$} & P1 leader & \multirow{2}{*}{$\begin{array}{c}\text { P1 intron } \\
-5276 \text { to }-39\end{array}$} & \multicolumn{2}{|c|}{ hunchback fragment } & \multirow{2}{*}{$\begin{array}{l}\text { Lines } \\
4(4)\end{array}$} & \multirow{2}{*}{$\begin{array}{c}\begin{array}{c}\text { Blastoderm } \\
\text { expression }\end{array} \\
\text { yes }\end{array}$} \\
\hline & -5839 to -5277 (563 bp) & & -5382 to +24 & (5406 bp) & & \\
\hline Megaselia & -1117 to -907 (211 bp) & -906 to -20 & -8000 to +2868 & $(11 \mathrm{~kb})^{\star}$ & $2(2)^{M S}$ & yes \\
\hline Platypeza & -2035 to -1765 (272 bp, P2) & -1764 to $-12(\mathrm{P} 2)$ & -6173 to +39 & (6212 bp) & $3(4)$ & yes \\
\hline Lonchoptera & -5119 to -4565 (555 bp) & -4564 to -88 & -4640 to -58 & (4583 bp) & $2(3)$ & yes \\
\hline Empis & -5882 to $-5450(433 \mathrm{bp})$ & -5449 to -10 & -5892 to +105 & (5997 bp) & 3 & no \\
\hline Haematopota & -1937 to -1646 (292 bp) & -1645 to -15 & $\begin{array}{l}-9000 \text { to }+105 \\
-1752 \text { to }+105\end{array}$ & $\begin{array}{l}(9 \mathrm{~kb})^{\star} \\
(1857 \mathrm{bp})\end{array}$ & $\begin{array}{l}3(2) \\
5\end{array}$ & $\begin{array}{l}\text { yes } \\
\text { no }\end{array}$ \\
\hline Clogmia & -3050 to $-2730(321 \mathrm{bp})$ & -2729 to -9 & $\begin{array}{l}-6872 \text { to }-3 \\
-2440 \text { to }-3\end{array}$ & $\begin{array}{l}\text { (6870 bp) } \\
(2438 \text { bp) }\end{array}$ & $\begin{array}{l}3(3)^{A P} \\
3(3)^{A P}\end{array}$ & $\begin{array}{l}\text { yes } \\
\text { yes }\end{array}$ \\
\hline Anopheles & -2464 to -2393 (72 bp) & -2392 to -2 & -5205 to +31 & (5236 bp) & 4 & no \\
\hline
\end{tabular}

* estimate, fragment was not completely sequenced. ${ }^{\mathrm{MS}}$ Cloning and fly lines established by Michael Stauber. ${ }^{A P}$ Cloning and fly lines established by Alexander Prell 


\section{A.2 Supplemental figures}

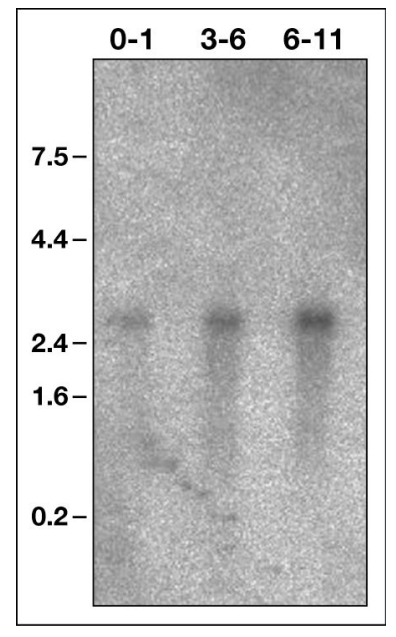

Figure S1. Northern blot analysis of Clogmia hunchback. The sampling covers the early embryogenesis up to the extended germ band stage. Each lane, $300 \mathrm{ng}$ of poly $\mathrm{A}^{+}$RNA were loaded. Poly $\mathrm{A}^{+}$RNA was prepared from pools of $0-1$ hour-old embryos, 3-6 hours-old embryos, and 6-11 hours-old embryos, respectively. After separation and transfer onto Hybond- $\mathrm{N}^{+}$nylon membrane, the RNA was hybridized to a radioactively labeled Clogmia hunchback cDNA probe, which covered the leader of the maternal transcript (351 bp) and parts of the second exon (747 bp). In all three lanes, a band is detected that corresponds to a transcript of about $2.8 \mathrm{~kb}$. As size standard, the 0.24-9.5 kb RNA Ladder (Gibco BRL) was used; fragment sizes are given in on the left kilo bases. The experimental data are a courtesy of Alexander Prell.

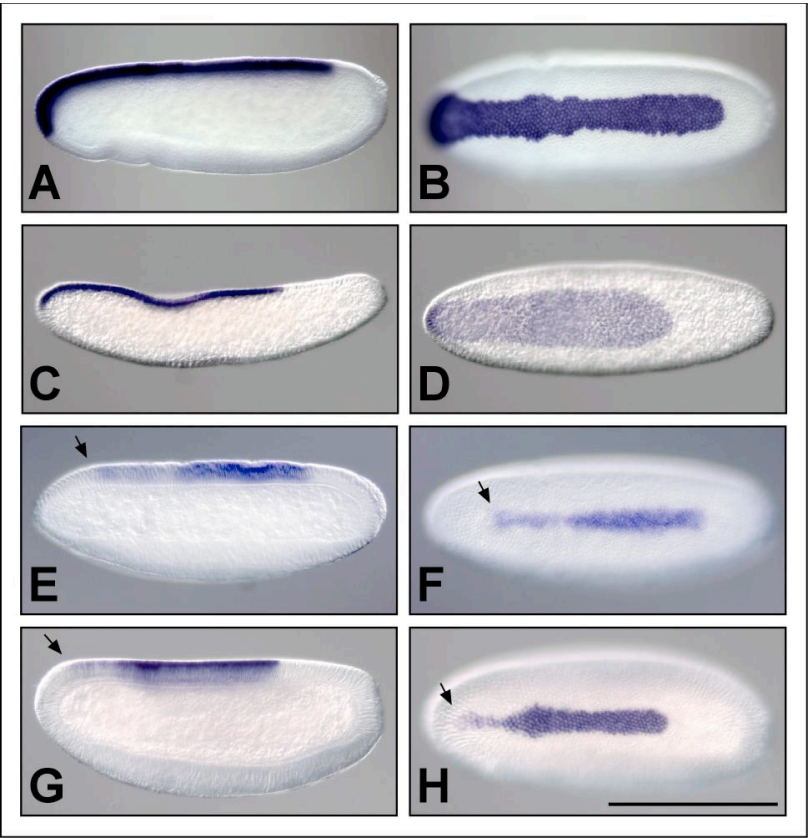

Figure S2. Comparison of dipteran zerknüllt expression. Expression of zerknüllt homologues was compared at the onset of gastrulation by whole-mount in situ hybridization in Episyrphus (A, B), Clogmia (C, D), Drosophila (E, F) and Megaselia (G, H). Each embryo is shown in a lateral view (left panel) and in a dorsal view (right panel), respectively. Anterior is to the left. (A, B, C, D) In Episyrphus and Clogmia, zerknuillt is expressed in a stripe along the dorsal midline and extends to the anterior pole. Similar to dorsal hunchback expression (Figure 12 E, F), in Clogmia the dorsal zerknüllt expression is slightly broader and extends less far to the posterior than in Episyrphus. (E, F, G, H) In Drosophila and Megaselia, zerknüllt is expressed in a stripe along the dorsal midline. Expression is absent at the anterior pole (arrow indicates anteriormost zerknüllt expression. Arrows hint at the anteriormost expression, which in Megaselia extends slightly more to the anterior than in Drosophila. Scale bar: 450 $\mu \mathrm{m}$ in $\mathrm{A}, \mathrm{B} ; 180 \mu \mathrm{m}$ in C, D; $220 \mu \mathrm{m}$ in E, F; $240 \mu \mathrm{m}$ in $\mathrm{G}, \mathrm{H}$. 


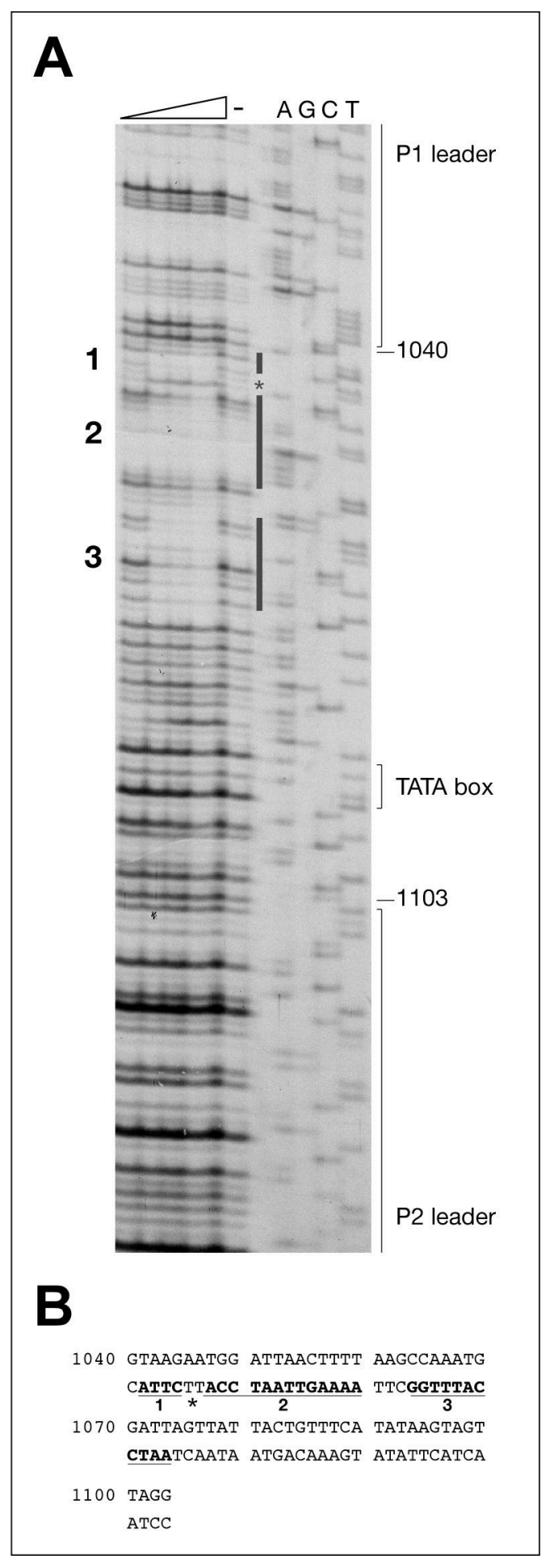

Figure S3. DNaseI footprint mapping of Bicoid binding regions in Megaselia hunchback regulatory DNA. A Megaselia Bicoid-GST fusion protein spanning amino acid residues 78-159 (including the complete homeodomain, Stauber et al., 1999) was expressed and purified as described previously (McGregor et al., 2001b). The concentration of active protein was estimated by gel-shift assays using a double-stranded oligonucleotide with a single Bicoid binding site (5'-ATCTAATCCC) as described previously (Shaw et al., 2002; Zhao et al., 2000). (A) The genomic region 838-1151 of Megaselia hunchback (SEQ09, Appendix A.3) was analyzed by DNaseI footprinting for the antisense strand as described previously (Bonneton et al., 1997). $0.5 \mathrm{ng}$ of labeled DNA were titrated. The triangle represents increasing concentrations of purified Megaselia bicoid protein $(5,0.5,0.05,0.005 \mathrm{nM})$. The negative control lane (no protein added) is indicated by "-"; bars indicate protected regions and the asterisk marks a hypersensitive site. The TATA box of the P2 transcript as well as exon sequence (P1 leader and P2 leader) are marked. The region between the 3' end of P1 exon 1 and the 5' end of P2 exon 1 corresponds to nucleotides 1040-1103 in with SEQ09. (B) Summary of the protected (footprinted) regions in front of Megaselia hunchback P2. Protected sites are underlined and shown in bold, the hypersensitive site is marked with an asterisk. The experimental data are a courtesy of Philip Shaw. 


\section{A.3 Sequences}

The sequences are color-coded. Genomic DNA is set in black letters. Sequences that belong to the putative open reading frame (ORF) are marked red. Putative untranslated regions (UTRs) of transcripts are marked blue. Numbers to the left of the sequences indicate the position of the first nucleotide in the row.

\section{SEQ01 Platypeza bicoid, cDNA.}

1 AgTTTAAGGC CGGCGGCAAA AATAGGCGCT AgTGCAGTAG GCAAAATGGC GCAACACCCG GACCAGAATT TCTACACCCA TCAACAACAg TACGGGTTTA 101 ACAATAACCA TCAACAAATG CAATTTCCAC CGCATTTCCG GACGCCGTAC GATTTTGTCA AAATGTTTGA CGAACGCGCG GTGGCTCTGA ATTACAACTA 201 TATGCGACCG TATATGGCTC ATCAGATGCA GCAGATGCAG ATGCAACAAA TGCAGCAGCA AATGCAACAA GGTTACCATG ATATGAACAA TTCGATGCAC 301 GACATGTTGT CCGAGTCGCT AGTCATGCGG CGTACGCGTC GGTTGCGCAC GACGTTTACC CAACAACAAC TGCAGGAGCT CGAGCAAGAG TTCCAGATCA 401 ACAAATATGT AACAGCGCTC CGCTTAGCGG ACATTACAAG CAGATTGAAT TTGGCAAACG CTCAGGTGAA GATCTGGTTT AAAAATCGGC GGCGAAAGCA 501 TAAAATCGAA GAGGCTCGCA TGAAAGAGCT CAAGGGCACA CTCCCACTTG GGTTGAATGT GTCGATTCCC AATTTGAATG GGTCCCTCAC CTCAAACAGT 601 CTGGACAGCT CACTTTCGGA ATCAGCGCCA CCTAGCGAAA CGAAAAGCGA ATCGCCACCG CTGCCGCTTA CACCAAATCC ACTAACACCG TCGCCAACCC 701 CGTCTGCTAC CTCAACACCA AGTGCGTCTG ATAAACAGTC GGACAATTCC AACTACGGCA ATCAGTTCTA TTACAACAAC AATAACAACC AAATGCCGCA 801 GTATTACCAA ACACCGCCGG CCACAAGCAA CCAACAACAG TTCGAGTTCC CGACCAAGGT TCAACAACAA AACGAAACAA GATACAACAA CAATAACAAC 901 AACTTCAGCC AGCAACAGCA ATTCAACCGA TTGGCATCCC AGGAGAAGCT CGCCGAGTTT GCCAAACAAC TAAAAATCAA ATCGGAAATG GCCGATTTTA 1001 ATTCGGCGGA ATTGTCGCCA AATTCTGAAG TGTACGAACC ACTGACACCC CGAACTGACA CGAGCCCACA TTCCGGGCAT TCAGACGAGA TCGATGAAAC 1101 TCTAAAGTCA AATCACGCTC ACACTCCGAC TGCAGCGGAG TTAAACGGCG ACGAGCCGCA ACCCGATGCT GCGTCCGCTG CCTACCAGGG CCAACCGATG 1201 TACAACAACA ACTCGAATAG AAGATGTGGC GACGAACAGA TGTTCGGCTA CAGATACAAC TAAACGAGTT GTTCTCTAAT TACCGTTATA AAATTGTTTA 1301 TAATCTCAGT GATTAGGTTT CCGACCTAGT ACATGTTTAG TTGATAAGCG CTTAGCCACA TAAGTTTAGT TTTAGTAACG GTTTCCATCT GCTAGTGATT 1401 TTTCGCTTCG CTAGTCTCTT CCGGTTTTCG GTCCATGAAT TCTGAAGAGC CTACCGAAGC CCATGGACCA TTATCGCTAC CAGATCGAAA CAAATTCACG 1501 ATTTTCGCAA ATTATGAAAA ATCGCAAAGA AAACAAAATG AAGGAAATCA TCCACTCAAC AAAGACGG

Source: Two independent 5' RACE products (1..374; 49..608), 3' RACE product (866..1568) and an additional PCR product (38..1539), all amplified from a larval cDNA template.

\section{SEQ02 Lonchoptera bicoid, cDNA.}

1 TTGAACGATT TGCGATAGTG ATAGTTGTGA TGGTCAAGTC AGGTTTTGAT TAAAAATATA AGAAAAGAAA AAGTTTGTGA ATAATATTTG ATTGAATTTT 101 AAGTGATTTT ATGTTTATAG TAGTGAATAA TTTAAGGAAT AGTAAAATTA AGGAATAATT TACATAAAAC TTATGTGAAA GATATGTGAA ATTTTTGTTT 201 TTTTTACATC AAAGCAACTC GCACATTCCA TACAAAAATG GCGCAACCGC CTGATCAAAA TTTCTATCAC CATCCGCAAC TACAGCAACT ACAGCTGCCT 301 ACGCAATTTC GGAATCCATT CGATTTGTTA TTTGACGAAA GAACTGGAGG TTTAAACTAC AATTATATTC GGCCATATAT ACCAACTCAA CCAGTGGTAC 401 CAGATGTTCG AAATGAAGCA GTACGTGCTG ATCCACTTGT TATGCGAAGA CCACGACGTA CTCGAACCAC ATTTACAAGT GCGCAAATTT CAAAACTTGA 501 ACAGTACTTC AACGAAAGTA AATACGTAAA CGCTTCACGT CTAGCTGAGC TATCTGGTAA ACTTAATCTT GGAAATGCGC AAGTAAAAAT TTGGTTTAAA 601 AATCGTAGAC GTCGATTGAG AATTGAACAA CTAAAACTGA AGGAACTAAA TGGATCAAAT GATACAACAC CAGCAGTCAG TGTTTCTAAG GATTTGTGTC 701 TTGCGTTGCC ATTAACTCCA ACAACTTTAA CACCTTCGCC ATCTTTAACA CCGACTAGTA CACCAAATAT AAGCGATCAG TACAGCGAGA ATTATACGTA 801 CAATCCGTAT ACTTATAATC CATATGTACA GCAGCATGCA TATGAGCAAC AAGTCAGAGC ACAACCAATG GCAACGCAAT ATTATCAGCA ACCATCAGCG 901 ATTAGTCAAC AGCTTACAAG AGATTTTCTA ACATCAATTA AAACGGAACC GGATTTCAAT TACAATAGTA CTCCTTATAT GCGAATGCCG GCCGCAGAAA 1001 CTATGGTGAA TTACACTAAA ATTCCTACTA AAAATTGCTA TTTACCCGAA CTGTCACCCA ATTCTGAAGT CTACGAACCG TTAACACCAA AAACTGAAGG 1101 CAGAGGAAGC CCAAAAATGG CAAATACATC AGATGAAATT AGCAATACAC ATTTAGTTGA TGCTAAACCA GAAGTTTCGT CGGATACAGC ATCACAGATA 1201 TATGAAATGA CTAAGTCAGT ACCCGAAGGT GGATACCAAT GCACCATGGA TTCGATATTG CAAGCATACA ATCAACATCG CAATACCAAT ACCAATAATG 1301 GTTACAATAC TCAGTTTGCA TTTTGCTTTA ATTAAGTAAA CAATCAAAAT TATATTAATA ACAAAATATA ATTAGTTATT AAGTATTAAT TATAAAAATT 1401 ATAATGTCTC AGTGAGGTTT GTTAGTTATT AGCTTAAGTT ATCGTTTAGA AAATAGGCAC TTACACCAAT TTTTTTCTCT TTTTTTGCAG GGTATTTTGT 1501 AACCAATTTA GCTCTAAGTT AAGATATACA TATTATTATT ATTTTTTTTT TTTATAGTTA TTTAAATGAT TTGATAATAA TTAGCTACGA AACTTAATCC 1601 AAATATTAGG TCCATGATAT TTGAATGAAG TTTTGTAGCT CATGGACCAA TATTATTATT TCACTGCTGG GGTATATATT TGCTTAGCAA AAACATAAAA 1701 AAGAAATAAA CAAATTATGA ATCTTTTTTA AGAATTATCA AAAATCTGAT AGATACTCTG AACAATTGCA GATTATTTCT CACAATTTTT TTTATTTGTT 1801 AAGATCCCAA CAATGCATTT ATTTAAAACA ATTGCAATGA AACTATATTT TTCGAAATAT TTAAATTATA AATATAATCT GTAATTAAAA CAGCGTTCCT 1901 CTTGCGAAAA ATTCAAAAAT GTTAATTTCT CGAAAAGATT GAGGCTTAGA CAATATGATA AAAAATTTTT ATAATGTGAA TTAATTTCAA AAAACTTCAC 2001 CGAAATTTTT AAATTTCTCT TAAATTAAAA TTATGTAATT TCGGATGAAG TTTTAAGAAA TATCTTGGAA TTGTTTTATT TAGATACATT TTTGATAAAG 2101 ATTTTTTGTT TGTTTTTTTT TTTTTTTAAT TTTTTTTTGT TTAATTTAGT TATATTAAGA TTACCAAATT TAGAATAAGT TATAATTCAA GTTGTTGTTG 2201 CAAGAATTAA ATGAATAGGT ACCTAAAACT CGTAAGGAAA ATTGTTTTTT TTTCAATATT ACTTTGTACA TTTAGTAATT TAGTATTAAG TTTTTCTATT 2301 TTTCTTTCTT TTTTTGTAAT ATTAATATGT TGATTAAT

Source: 5' RACE product (1..509), two independent 3' RACE products $(550 . .1192 ; 909 . .2338)$ and an additional PCR product (488..1940), all amplified from a cDNA template of adult females.

\section{SEQ03 Episyrphus orthodenticle, cDNA.}

1 GACTTCTGTA CGTCTTGGG TCTTGGTCTA GCTTCTAGCA TATATAAATA TAAATTATAA GGTGTAATGT TATGATTGTG ATAAACTTTA AGAACTGTAA 101 ATTTTATGAC ATTTTATATG GCCCGCTCAg TAGCCTGTTG TTGATAAAGC TTGATAATTT ACTATTTCCA TTCAATTCCA TTGATAAAGT TGTTGAAGAT 201 AATTCACAGT ATTCTTACAT CACAATATTC CGCCGCAGCC GTTTAGCAGT TGTCGAAGTA TATACAAAGT TTTTCTTTAG TCGATTGTGA AATAAAAATA 301 TAAAAAAAGT GCAAGAAAAT CATAGTTCTT CAAAACTTGA TTCGAACGTG TTCCTCCAAA AAAATTCTCA TCTTTTCTGA TAATCAACAT ATCGAGTGTT 401 AAATAATAAT AATAATAATC ATCATCATAA CAACAGCAGA AGCAATATAA CAGCAGCAAC AAATCAGAAA CACAAGTAAA ATAAATTCAT TCACAGTGGC 501 GCTGCATTCA ACAGAAGCCC TCATGGCAGC GGGCTTTTTA AAATCTGGTG ATTTAGgACC ACATCCGCAT AGTTATGGTG GTCCGCATCC ACATCATTCT 
601 GTACCACATG GACCATTACC ACCGGGCATG CCAATGCCAT CATTAGGACC CTTTGGGTTA CCTCACGGTT TAGAAGCTGT TGGGTTCTCC CAAGGTATGT 701 GGGGTGTAAA TACCCGCAAA CAAAGACGCG AACGTACAAC ATTCACACGA GCGCAATTAG ATGTATTGGA ATCGCTATTC GGCAAAACAC GATATCCTGA 801 TATTTTTATG CGTGAAGAAG TTGCTTTAAA AATAAATCTA CCCGAATCAA GAGTACAGGT TTGGTTCAAA AATCGACGCG CCAAATGTCG TCAGCAACTC 901 CAACAACAAC AACAATCCAA TTCGCTCAAC AGTTCCAAGG GCAATAGTGG TAATGTGGGA TCGGGCGGTA ACTCCGGTTC CAGTCGCAAT TCATCGAATA 1001 GCAATAATAA CAGCGGCAAC GCCAACAATC AAAATAGCTC TAGTGGCAGC GGCAATTCAG GAACAAACTC ATCCAACACT CCAGCGAAAT CGAGCAATAA 1101 CAACAATAAC AACAACAAAT CATCGGCGAA TTCAGCGCCC GTCACACCGA TGTCGCCACA GAGCGCCGCT GTGGCACATG CTGCTGCCGC AGCTCAGTCC 1201 GCTCAATCGG CCCACCACTC AGCTGCCGCT CACTCTGCCT ACATGTCCAA CCACGACTCG TATAACTTCT GGCACAATCA GTACAACCAG TACCCCAACA 1301 ACTACCAGAC ACCCAGCTAT TACTCGCAAA TGGAGTATTT TAGCAATCAA AATCAGGTCA ACTATAATAT GGGACATTCC GGGTATAGTG CCTCGAACTT 1401 TGGCCTCTCG CCGAGCTCAT CATTCACAGG AACCATGTCC GCGCAAGCCT TCTCCCAGAA TGGCCTCGAT TACATGTCAC CCCAAGACAA GTATGTGAAT 1501 ATGGTGTAGG AGATCCTACA GTCGGCCGCT GGCAATGTGG CTATAGAACG GATAGGTGGC GACACCCAGG CCCTCGAAAA CACATATCCT AAAAATCCTC 1601 GAG

Source: 5' RACE product (1..763) and 3' RACE product (740..1603), amplified from an embryonic cDNA template.

\section{SEQ04 Episyrphus hunchback, cDNA, P1 transcript.}

1 AGTCGTGTTC GAGACATCAC AACGCAAAGG ATGCGTGCGA TCACGGTTTA GATATTTATA TACAAATAAC AAAATTAAAA TTTTAATAAT ACATGCATTT 101 TTTCGTCTTC TATAAACAAA CAAATATAAT TATTATTATT GCTCTCTCAC AACTGTCAAA TCTTGGAAAA TAAATTTAAC AAGTGTATTT ATTGAGACAT 201 TACAAAAACG AATCAACTGG ATTACCATTA TATTTTTTTT AATGAAATCA TATGATTTTG TAGATTTATA AACAATAATT AACTGTGAAA TAAAATATTG 301 CCAACAAAAT CAGAACAGAT CAAAGTGTGA ATAAAAGAGT TAAAAAAAAA TCTTTTTGTT GTTGTAAGTG ATGATGCTGG CGCGTATTCG AAAATAACGG 401 ATATTTAGTC AGAATAAAAG AGAAAACTGT TTCATATTAA ACTAAAAGTT AATATTTAAA TTTCGAAAAC GGGGAATATT AATTCTGTAA ACGGAGAGAA 501 GTCTGAGAGA GGGCAGTCGC AGAGAAGCAA AGAAAACCGC TTTGAAACAA AATTATTATA AAGTTTCTAA TTCAAATTAT ATACCCAGAA GCGGCTCCAA 601 GATGCAGAAC TGGGATTCAA TGCAGCCAGC AGCCAATTAC GAGCACAATT GGTACAGCAA CATGTTCCAT CAGACAATCA AGCAAGAGCC TTCACAATCC 701 ACCACCCCCA CCACAAATCA ACTGGAGCAT TATCTCAACA TGAAACAGCA GGAGCTGTCA TCGGCGATGA CTCCTTCGCC ACGAGTTCCC GACTCAAATG 801 TCAATTCGGC GATGATAGGg GGCGATGTTG GTAACAATAC ACAGCATTAC TTTGACAGCT CAACGGGAAT GTTGCATCAA CATCATCCAC TCGGATTTAA 901 TCCACTTACT CCACCAGGGC TGCCAAATGC TGTCTTGCCT TCGATGTCAC ATTTTTATCA ACAGAATACC CATCAAAGTG TCAGTACAGG ACATCCTGTT 1001 GAATCTGTGA CCAAAATGGA TCAACAGTCT ACGAATAACA ACTCACTTAC ACCGAGAAAT ACTCCACCGA TGGATGTGAC TCCACCCAAG TCACCCAAAC 1101 TATCACTAAT GATTTCCACT TCGAGTGAgT TAGATCAAGA TGTGATGTCA TCTAATTCTA GTGAGGACAT GAAATACTTA GAAAGCGAGG ATGACGAAAG 1201 TATCCGCTTG CCAATCTATA ATTCACATGG AAAGATGAAG AATTACAAAT GCAAGACTTG TGGATTTCTG GCTATAACAA AAGTGGCCTT TTGGGAACAT 1301 GCACGTTGTC ATATGAAGCC TGAAAAAACA CTTCAATGTT CCAAATGCCC TTTTGTCACC GAATTAAAGC ACCACCTTGA ATATCACATT AGGAAGCACA 1401 AGAATTTAAA GCCATTCCAA TGCGACAAGT GTAACTACAG CTGTGTTAAC AAGTCTATGT TGAACTCCCA CCGGAAGTCA CACTCGTCGG TGTATCAGTA 1501 TCGTTGTTCT GACTGTGATT ACGCCACAAA ATACTGCCAT TCTTTTAAAT TACATTTAAG AAAGTACGAC CATAAGCCCG GCATGGTTTT AGATGAAGAT 1601 GGTGCACCCA ATCCATCTGT TGTAATTGAT GTCTATGGAA CACGACGTGG GCCAAAAATG AAGTCCGGCA GGAAAGAATC AACTGGGGCG TCAAAGATGC 1701 CACAATTGAG TGCAGCCTTG CAAGGATTTG CCCTTAATCA GCACAACAAT CAACACAACA TGCCTGCTTC TCCAGCCAAA AGTACTGCTT CGTCATCGTC 1801 AGAGATAGTT CCAAATACCC AGTCGAATAT TCAAGCTAAC CACCAGCATT TACAGCAGTC AACTTCCACT CAACAAGAAC AAATCGAACA ACACCATCAT 1901 CAGCAACAAC AGCAGCAGCT TTCTAATCTC ATCCCCTCAT CGTTGTCAGC GATTCTGCAA CAACAAAGAA ACATACCATT TTTTCCTTAT TGGAACCTTA 2001 ATCTTCAAAT GTTGGCTGCC CAACAACAAG CTGCTGTTTT GGCCCAGTTA TCGCCAAGGC TTCGTGAAAC AGCATTACAA AATCTGCAAG ATAAGCAAGA 2101 AGACAAAAAT GCAAGCTTTA TAAATGAAGA TGACGAAGAG CATGATGAAA GTTGTGACGG AACAGCTATG GATCTCACGG CCGCTACTCC AACTAAAAAC 2201 AACGAAGACA TCAATTCTTC TATTGTAAAC CTTAAATTGA AAGAGGATGA CCAGCATGAg ACTCCTCTTA TAAGTTCGTC CAATCAGTTC CGTCGCAAAG 2301 GTCGAGCACT AAAACTTGAT GCAGCTCTTC AAGCTAAGGA AAATTCCTTG AGTCCAGAAG AGAAGCCTCG ACTTAGCCCG AATCCAAATG AGGTTCCGTC 2401 ATCTTCATCA TTCGATGATC CATCAAAAgA GAGTGAAACC TCACAATCTA ATGAAGACTC GTCAAGGCCC TCCAACAGCA CCTCAAATCC AACATCAACT 2501 CCCACAACCC ACTCCACGTC CACAACCAAC AAATCACCTC CATCRGGCGC CATTTTCGAA TGCAAATATT GTGATATATA TTTCCGTGAT GCAGTCCTTT 2601 ACACTATTCA TATGGGCTAT CACAGTTGTG ATGATGTATT CAAGTGCAAC ATGTGTGGTG AAAAATGTGA TGGTCCCGTC GGATTGTTCG TCCACATGGC 2701 ACGCAATGCT CACTCTTAAA AGAGAGTTCA CTTGATAAAC CCCTTTTTTG TTATTTCATA GCTCGTTTTT GGGAGATATT CAAAATCAAC CAAAAACAGA 2801 GTTTCGTTGT CAAAGATTGT AAATATTAAC ATAATAAAAT TTATATTTTG TTATGAAAAA ATTAAATATA AGTAAA

Source: 5' RACE product (1..897), 3' RACE product (1478..1885), and an additional PCR product (498..2876), all amplified from an embryonic cDNA template.

\section{SEQ05 Episyrphus hunchback, cDNA, partial P2 transcript.}

1 GAAATCCTTA AAATGTGTTT CTTGGTGCAC AATAAAAATT TCTAATTCAA ATTATATACC CAGAAGCGGC TCCAAGATGC AGAACTGGGA TTCAATGCAG 101 CCAGCAGCCA ATTACGAGCA CAATTGGTAC AGCAACATGT TCCATCAGAC AATCAAGCAA GAGCCTTCAC AATCCACCAC CCCCACCACA AATCAACTGG 201 AgCGTTATCT CAACATGAAA CAGCAGGAGC TGTCATCGGC GGTGACTCCT TCGCCACGAG TTCCCGACTC AAATGTCAAT TCGGCGATGA TAGGGGGCGA 301 TGTTGGTAAC AATACACAGC ATTACTTGGA CAGCTCGACG GGAATGTTGC ATCAACATCA TCCACTCGGA TT

Source: 5' RACE product, amplified from an embryonic cDNA template.

\section{SEQ06 Episyrphus hunchback, cDNA, partial P3 transcript.}

1 AGTTCGTGAT CGGTATCTTG TTGTAAAACA CCAAATCTTC GTTCCGTTAT CAGGAAAACA ATTTAAATAA CGAAATTGA TGTCAAAGTG TATTTCTGGC 101 CAAGGAACAA ATAATATAAA AAAAGTTTCT AATTCAAATT ATATACCCAG AAGCGGCTCC AAGATGCAGA ACTGGGATTC AATGCAGCCA GCAGCCAATT 201 ACGAGCACAA TTGGTACAGC AACATGTTCC ATCAGACAAT CAAGCAAGAG CCTTCACAAT CCACCACCCC CACCACAAAT CAACTGGAGC ATTATCTCAA 301 CATGAAACAG CAGGAGCTGT CATCGGCGAT GACTCCTTCG CCACGAGTTC CCGACTCAAA TGTCAATTCG GCGATGATAG GGGGCGATGT TGGTAACAAT 401 ACACAGCATT ACTTTGACAG CTCAACGGGA ATGTTGCATC AACATCATCC ACTCGGATTT AATCCACTTA CTCCACCAGG GCTGCCAAAT GCTGTCTTGC 501 CTTCGATGTC ACATTTTTAT CAACAGAATA CCCATCAAAG TGTCAGTACA GGACATCCTG TTGAATCTGT GACCAAAATG GATCAACAGT CTACGAATAA 601 CAACTCACTT ACACCGAGAA ATACTCCACC GATGGATGTG ACTCCACCCA AGTCACCCAA ACTATCACTA ATGATTTCCA CTTCGAGTGA GTTAGATCAA 701 GATGTGATGT CATCTAATTC TAGTGAGGAC ATGAAATACT TAGAAAGCGA GGATGACGAA AGTATCCGCT TGCCAATCTA TAATTCACAT GGAAAGATGA 801 AGAATTACAA ATGCAAGACT TGTGGATTTC TGGCTATAAC AAAAGTGGCC TTTTGGGAAC ATGCACGTTG TCATATGAAG CCTGAAAAAA CACTTCAATG 901 TTCCAAATGC CCTTTTGTCA CCGAATTAAA GCACCACCTT GAATATCACA TTAGGAAGCA CAAGAATTTA AAGCCATTCC AATGCGACAA GTGTAACTAC 1001 AGCTGTGTTA ACAAGTCTAT GTTGAACTCC CACCGGAAGT CACACTCGTC GG

Source: 5' RACE product, amplified from an embryonic cDNA template. 


\section{SEQ07 Episyrphus hunchback, genomic.}

1 GAAGTCTGAG AGAGGGCAGT CGCAGAGAAG CAAAGAAAAC CGCTTTGAAA CAAAATTATT ATAAAGGTAA GTTTTTGATT ATTGGAAAAA GTTATTTTTT 101 TCTTTAAATA TTTTATTTTA TTACTTTTAT CGGTCGATCA ATCGTTTTCA CTAAATTTTA TTGCAAGGTC GTTTATAATT TATATTTTCG CTGAGTTTTT 201 GAGCATATGA AAATTTATTC AGCTATTTGA ATTCGAATCT TTAAGACATT GTTTAGCAAT ACTCAAAGTC TGACATTTAA ACCTAGTAAC GTAACCGGGA 301 CTCTTAGAGT TGATCTGTGC ATCTTGAAGC TGAATGGGCA ATTTACAATA ATCGTATAGC ACTTTTTTTG TTGATTCTCG TTAGAAGTTC AGGTACCTAC 401 CCTAAATAAT TGTTTACAAA ACCTGAAATT AAATTTGTAT CTTACAAAAA AGTTGTGCTG TAAAATAGAC TGACTTTCCC TTTTCATCAG TAATTTAGGA 501 ACAATGAAAG ATTCTAATAA AGTTTTCAAA ACCTTTCTTT TTTGTTTACT TTAACATTAT AATAATTTAC GCTATTTAAA GTTTTTAAAG AGTGGTTCTG 601 TTATCGTGGA TGCAACATTC AAACTGATCA GGTAGAATAA TAGGTAATAA GCCTTGAAAG AATAATTTCA TCAACAAGTT TATTTTGTTT TTTCGTACCT 701 AAATAAAATT CAAATATGTA CAAATGTTGC ACCCGAGATA ACTGAATCCC TAATTTAGCT GTTTGCTAAA ATGTGAAATT GATACTTTTA ACTATAATTC 801 TATACTTAAC ATGCTGATTT TTAATCTTAT TCCATAAAAA ATTGCTTAAA AAATAAAAAT CACGTGAAGA CTTGAGAGCA CTCGCTATGC AGAATATAAT 901 GGAACACCTG CTTTATATAG TTTCAATTGC TAAAGCTACA TAAATCCCAC GAAATATTAA ATAAAACGAT GATCTAATCT TCCTTTTAAG ATGCACCATT 1001 TATACATTAA TAGATATATG TACACACATA TGTATATATC GAAAACAAAC TTATAAGAAT GTTAAAATAA ATCCCAAAAA CCATTAAAAA CAAATAAACA 1101 AACAAAAAAA AACAAAGATT CTCACAGAAA AATTTCTATT CTTGTGGTTA AAAAATTTTA CAAAATTACA GTTTTTTTTT TAATTTGCAA AATTTTATTT 1201 TAAAATCATT ACTCACAAAC TAAAATGCAG CGTGTATAAC CTGCCCCTAC CTACCTATTA CCTTCCATGC CTCCACATGT AAAACAATTT TTTGTTGCTG 1301 CACAAAAAAG TCTGCGGAAT ATAGTTTTTG TCTCACAACA AAATTAGAAG CAGCCATAAT TCAAAGTCAC TAAAAAACTT GGCGCAAAGC CTCTGTATTC 1401 ACTGAGAATC ATATCATTAC CGTCATCATC GTCAAGATTG TGTTTTGCTA GTTTTTTTTT TTTCCTTCTC AAATCGGTAC ATTCAATTGC AATGTTGTCT 1501 AAAATCACCG ACGGCAAAAG TGTGCATCAT CAAATTTAAG GAACACAAGA AAAAAATAAA TCTGGCTTTA ATTTTTTTTA TAGAAAACAA AGATTTTCAA 1601 AAATACGCAG ATACAATTTT GCAGCGTGTG TATTTTTCAG ACGTTTTTTC CTCCCCGCCC ACCGAGAACT TGACCGGAAC ACAACCAAAC TAGAAAAAAA 1701 AGATGAAAGA GAAAAAGATT TTTGCACTAA AATAGTTTAA AAATATATAA AATATAAAAA CAAAAAGAAA AAACAAAACT AAAATGTATC TTCTACAGGA 1801 AAACTTTTTC ATATAACTAA AAGAATGTAT CTTAGATTTT TTAAAATACA TTTTTTGTTT ATGCATCGTT TGATGCATTT TTCGTTTATA TGGTTGCAAG 1901 GCAGTATCGG TGAAGCAACG ATGAGAAATG AAGATTCAAC AAGTCGATTT AGATGCAAGA AGATTCTCTG ATAGATAAAT TTTTCGAAAA TTGCTTCATT 2001 AATTTTTATA AAAAAAATAG AAAAAAAGAT ATAAAATGTA CACTTGGCCT TTGAGATCAG ATGTGCACAT GTGGGCGTAG TCGGCAATTT TTTTTTCTTG 2101 CAATAAGGAA AGTATGCACA TATTTTTTCC GTTTGACAAC ATATCGCAAA ATAATTTTAT AAAATAAAAC ATCTTAATTT TAACTAAAAT AAAAAAAAAA 2201 AATCGCTTAA GTCATTTGCA AAATACATGG CTATGTAAAC ATAGATGCGG TTAACTAAAG TGGTGAAAAA TCACATCAAA CTTTAAACCG AATTCTTTTA 2301 CTTTATAGAC CAAGTCATTC TTAATTGAAT AGGTGTAATT TAAAATCTCT TCTTTTTTTT TGTTGTTGTG TCAGCTTTCA GGTTAAAGGC TGGCGCTGGG 2401 TTAAACTATA AAATTTAACC TAGCGTTAGC CTTTAACCTG CTGCTGCTGG TTATTGGTAT GGTGATGTTG AATTCAGTTC ATTATTTAAG CGAGATTTAG 2501 ATTTGATTAT TGGTAAGGTG AGTTTTATTT TTTACCAACA CTCAAATAGA TATTTTACAT AGTTTCCAAG TCCTGTCATA TGAAAGTAAT TGATATTTAT 2601 GAAATTAAAT TCTTAACTCA TTGCATCTGG TTAATACCAA GTACTTCACT GTTTTGTTTT TTCTTGTTAA CCGTTATCTT ATATTATATA TAGCTTTAAC 2701 ATAATTATGT TTTTGCTTCC AAACCTATTT AAAATGTTCC TTTATCTTGT TGGCACCTAA GGGAATTGTA ACTTCAAACC TACTTTTTTT TTTTAAGTTA 2801 CGTATGTAGC TTAAAATATT GCTGAATTTA AATTCATACT AAGCGGATAG ATAACGTAAG TGGGCGATCG AATAAAAAAA AACTAAGAAA AATTACCTGA 2901 CACATAAAGG CTAAATATAG AATGAATTAA GAAATTATTG TCTTAGACCA ATTTCGAGAA CGACGCTTAT ATTATATAAT AAAATCCACT TTTTCCAGTG 3001 AACTCTTCAT AAAATTGTTA TCCTGATAAT TCGAGCGAAT CAAGGAAAAG AAACTTCTTT TGATTATTTT TCATGAACAA TTCTAATACA TTAAAAAAAT 3101 CCTTGTGTTA TTTAGACAAA AAATATGGTT TTTTAAGTAG TTGAAATTAT ATGTAAACTT AAACTGAAAG CCATCTAACT GCAACCTGTT AGTTCGACAT 3201 TATAATGATT TCGATCATTT ATAATAAATA CAATCAGCTT TCAAATCTAA ATAATATTTT TACATTACAA ATAATATCGT ACTACATTTA TGTACACACC 3301 ATAAATTGTG TATCTAAAGG ATTCTTTGTT AAATATCATA TAATTTTCTC AGTGAGACTT GATCTGATTT TCTACCTGTG TTAAAAAGGT AATTTTTTTC 3401 AATAATCCTT CTATATTTGC TTTTGCTCAA AAATCAATGA ATATTGGGGT GTGCACTTTC GTTTTTCTTT TTGTAAGTAT GATGTACTTT TTTGTGAAAA 3501 CCGTGACGAT ATATCTTGAA AATTCTTGCA TACACACTAT AAATAACAAA TAATATGAAA ATTTATAGGA AATCCTTAAA ATGTGTTTCT TGGTGCACAA 3601 TAAAAAGGAT GTTGATTTTT CAAACCGATT TGCATTGCAA GATGTTTGAC TTTTACTTTT ATGGTTACCT ATGCATACTT TTTTATTTTT TTTGTTTTTG 3701 TGTTCGTGTT GACCCTAAGT CATGAAACTT AGTAATTTTG AAAGTAATTT TTGACTGGTC ATTGGGTTTT TATGAGTAGT GAGTTGATGT CTTTTGAAAA 3801 AGATTTATTT TTTCTCAAAT AATGGTTTTA AATTCTGAGA AAGAAATTAA CAAAGGAGAC AAGTCATAAA GGTTTTAAAC AAATATTCAT ATTTGTAAAT 3901 AGATATGTAA GGAGTATTAG AACCAAATGC TTTTTTCCTG GTCATTTTTT TTTTTGAAAA ACAGGTAGGT AGGTAAAATT TCACTGGACA ATTTGAAGAA 4001 CAGAAATTAG TCTTTTTTTA ATATGATTTA GGTAGCTATA TATCACAAAC ACATAATATT TTTTTTTAAG TAAAATAAGT CAGACAAACA CCTGATTAAA 4101 AAATTTTGGA ATACAAAATG TGGTAATCCA CTATAGAAGC CTTCAAGAAT ATATTAAAGT TGAGCTATTT TTCGAGGAAA AGCTGTGCTT CCAGCAATCA 4201 GTCATACTTA TTTCATTTAA AAAACTTTAT ATTTTTAAGA TTTGAGTAGG TATGTATCTA AGTACCTCAC CAAATTATAG CCATAAATAT TTTTCTCATT 4301 ACCTACATTG CTTCTGTTTG CTGTAAGCCT AATTCGTTAT ACTTTTGTAC ACACATAAAG TTATTAAATC AACTCTACTT AAGAAACCAA CCAATCAGGA 4401 ATTGCCATAA ATAATTGAGT AGAAAAAACA GTGATCCTTT ACTTTGGCTT CCACAGAATA TTTTAACATT TACCTGCGCG AAACCGGGAT TGAAAATTAC 4501 TGATTTTTAC CTGCACTTTT TCCTGCCTTT AAATAAGAGT GAATGTCAAT GATATGACTT AGTTCGTGAT CGGTATCTTG TTGTAAAACA CCAAATCTTC 4601 GTTCCGTTAT CAGGAAAACA ATTTAAATAA CGAAATTTGA TGTCAAAGTG TATTTCTGGC CAAGGAACAA ATAATATAAA AAAAGGTACA AATAAATCAA 4701 TAATAAAATG GAATGTTATT TCCGATACTA AAATATTCTA AAACAAACGA ATCTGCTTCA ATACCATGTA AAAACCTCAA ATGGATTTTT CGCTTGCTTC 4801 TCATTCTCGA GAATTGTGCT TAGCGCGTGG ATATACATAT GTATATGTAT TTAGTACTTT ACTTTCCTGA TTTTATTTGA AAGCAGGAGA AAAACAATAA 4901 AAATATCCCC TTGCTTCCCA AAACATAAAA AAAAAACATA AAACGAAAAA AGGACCAAAC AAAAAATATC ATCCAAAAAC TATTTCAAAA AGAACTATAT 5001 GCATACATAT ATAGTAATCG CTTTTCAGTA CTTCTTCTTA TGTATGAAGT CCACCCCAGA AACTACATAC AGGGGGAGAG ATTTCCATGA ATTCCACCCT 5101 TATATACCAT TGCTTTATAG ATTATTACAT TCGGAGGGGT GACGTTTTTT CGTGGACACG CGCAGAACGT GTGCCAAAAA AAAACAAACA ATTGCAGATA 5201 AAAGGGGTT CAAATGATAA AAAATGGCGT AGCTTAAAAA AAACCATAAA AAATGAACTC ACAAAATGGC GCACATTTAT TCATCTATAT ACTTTTCTCT 5301 TTATCTTTAC AGTTTCTAAT TCAAATTATA TACCCAGAAG CGGCTCCAAG ATGCAGAACT GGGATTCAAT GCAGCCAGCA GCCAATTACG AGCACAATTG 5401 GTACAGCAAC ATGTTCCATC AGACAATCAA GCAAGAGCCT TCACAATCCA CCACCCCCAC CACAAATCAA CTGGAGCATT ATCTCAACAT GAAACAGCAG 5501 GAGCTGTCAT CGGCGATGAC TCCTTCGCCA CGAGTTCCCG ACTCAAATGT CAATTCGGCG ATGATAGGGG GCGATGTTGG TAACAATACA CAGCATTACT 5601 TTGACAGCTC AACGGGAATG TTGCATCAAC ATCATCCACT CGGATTTAAT CCACTTACTC CACCAGGGCT GCCAAATGCT GTCTTGCCTT CGATGTCACA 5701 TTTTTATCAA CAGAATACCC ATCAAAGTGT CAGTACAGGA CATCCTGTTG AATCTGTGAC CAAAATGGAT CAACAGTCTA CGAATAACAA CTCACTTACA 5801 CCGAGAAATA CTCCACCGAT GGATGTGACT CCACCCAAGT CACCCAAACT ATCACTAATG ATTTCCACTT CGAGTGAGTT AGATCAAGAT GTGATGTCAT 5901 CTAATTCTAG TGAGGACATG AAATACTTAG AAAGCGAGGA TGACGAAAGT ATCCGCTTGC CAATCTATAA TTCACATGGA AAGATGAAGA ATTACAAATG 6001 CAAGACTTGT GGATTTCTGG CTATAACAAA AGTGGCCTTT TGGGAACATG CACGTTGTCA TATGAAGCCT GAAAAAACAC TTCAATGTTC CAAATGCCCT 6101 TTTGTCACCG AATTAAAGCA CCACCTTGAA TATCACATTA GGAAGCACAA GAATTTAAAG CCATTCCAAT GCGACAAGTG TAACTACAGC TGTGTTAACA 6201 AGTCTATGTT GAACTCCCAC CGGAAGTCAC ACTCGTCGGT GTATCAGTAT CGTTGTTCTG ACTGTGATTA CGCCACAAAA TACTGCCATT CTTTTAAATT 6301 ACATTTAAGA AAGTACGACC ATAAGCCCGG CATGGTTTTA GATGAAGATG GTGCACCCAA TCCATCTGTT GTAATTGATG TCTATGGAAC ACGACGTGGG 6401 CCAAAAATGA AGTCCGGCAG GAAAGAATCA ACTGGGGCGT CAAAGATGCC ACAATTGAGT GCAGCCTTGC AAGGATTTGC CCTTAATCAG CACAACAATC 6501 AACACAACAT GCCTGCTTCT CCAGCCAAAA GTACTGCTTC GTCATCGTCA GAGATAGTTC CAAATACCCA GTCGAATATT CAAGCTAACC ACCAGCATTT 6601 ACAGCAGTCA ACTTCCACTC AACAAGAACA AATCGAACAA CACCATCATC AGCAACAACA GCAGCAGCTT TCTAATCTCA TCCCCTCATC GTTGTCAGCG 6701 ATTCTGCAAC AACAAAGAAA CATACCATTT TTTCCTTATT GGAACCTTAA TCTTCAAATG TTGGCTGCCC AACAACAAGC TGCTGTTTTG GCCCAGTTAT 6801 CGCCAAGGCT TCGTGAAACA GCATTACAAA ATCTGCAAGA TAAGCAAGAA GACAAAAATG CAAGCTTTAT AAATGAAGAT GACGAAGAGC ATGATGAAAG 6901 TTGTGACGGA ACAGCTATGG ATCTCACGGC CGCTACTCCA ACTAAAAACA ACGAAGACAT CAATTCTTCT ATTGTAAACC TTAAATTGAA AGAGGATGAC 7001 CAGCATGAGA CTCCTCTTAT AAGTTCGTCC AATCAGTTCC GTCGCAAAGG TCGAGCACTA AAACTTGATG CAGCTCTTCA AGCTAAGGAA AATTCCTTGA 7101 GTCCAGAAGA GAAGCCTCGA CTTAGCCCGA ATCCAAATGA GGTTCCGTCA TCTTCATCAT TCGATGATCC ATCAAAAGAG AGTGAAACCT CACAATCTAA 7201 TGAAGACTCG TCAAGGCCCT CCAACAGCAC CTCAAATCCA ACATCAACTC CCACAACCCA CTCCACGTCC ACAACCAACA AATCACCTCC ATCGGGCGCC 7301 ATTTTCGAAT GCAAATATTG TGATATATAT TTCCGTGATG CAGTCCTTTA CACTATTCAT ATGGGCTATC ACAGTTGTGA TGATGTATTC AAGTGCAACA 7401 TGTGTGGTGA AAAATGTGAT GGTCCCGTCG GATTGTTCGT CCACATGGCA CGCAATGCTC ACTCTTAAAA GAGAGTTCAC TTGATAAACC CCTTTTTTGT 7501 TATTTCATAG CTCGTTTTTG GGAGATATTC AAAATCAACC AAAAACAGAG TTTCGTTGTC AAAGATTGTA AATATTAACA TAATAAAATT TATATTTTGT 7601 TATGAAAAAA TTAAATATAA GTAAAAAAAT ACTGTTGTCC AAAATTGTAC ATACTATGAA TTTAAAAATC AAAACATGTT GTTTTTTATA AGTTTTTATA 7701 AGTATTTATA TAAAAACATA AATGTTGTTT TTTTTTCTTT TAAAATAAAA GCAAAACAAA CAAACAAAAT AAAATATTTA TAAAACCAAA TCTATTTTCA 7801 AAAAAATATA TATATTTTAT TACAAAACAT ACAATATATA AACATATATA AACTTCTAGG ACTAAGTTTA AAAGTTAAAG AAAGAAATTG ATATATCAAA 7901 ATAATATATC TGTTTCTATA AATGAATATA AAATGTAATC ATTCGCTTGA ATGTTTCGAA AAAGAAAATA ATTAATGTAA AAACTATTGC GAAAACAGGA 
8001 AGCAAAATCT TAAATTGTAG TCTATTTTTT GTAATTCCTA ATTTTGTAAT TAAAAAGAAA CTTGTTTGAA TCATAATTTA TTTTTTTTTT TACTTTATTT 8101 AGGTTTTTCT ACTTCTACTT CTTCGCTATC GAAAAAAACA AATATTTTTC TAGTCAATCA GGGAAGCCGT TTTATTCTTA TTATAGCCTC TAGGGGTTCT

Source: Two different PCR products (1..5352 and 4568..6207), both amplified from independent genomic DNA templates, and phage Eba-hb ph10 (5433..8200). Alignment with cDNA sequences: Positions 1..66 correspond to parts of the first exon of the Episyrphus hunchback P1 transcript (SEQ04), positions 3568..3606 correspond to the first exon of the P2 transcript (SEQ05), and positions $4561 . .4685$ correspond to the first exon of the P3 transcript (SEQ06). Positions 5313..7625 are presumably common to the second exon of all three Episyrphus hunchback transcripts. Three putative polyadenylation signals (Birnstiel et al., 1985) were identified in the genomic sequence $(7582 . .7587,7744 . .7749,7768 . .7773)$, and two putative nanos response element (NRE) sequences (Wharton and Struhl, 1991) were identified (7547..7578, 7626..7657).

\section{SEQ 08 Megaselia hunchback, cDNA, partial P2 transcript.}

1 AAAGGTTGTA GAACCAAGTC AGTTGAAGCA GAGAAATCGA AGAGATAGAT ATACAACAAA AATCAAAATG CAGAATTGGG AATCATTACA ACAAACAGCT 101 TCGTATGAGC ATAATTGGTA CGGAAATATG TTTCCAGCCA CACAAATCAA AACAGAGCCT CTGGAGCCAT CCAGTCAACC ATCGCAATTG GAACAGTATC 201 TCACATCGAT GAAACAACAA CAGCAACACA CCAACGAAAT GAATTCAATG ACTCCATCAC CAAGAGGTGA GAACGAAACA CAAAGTTTCT TCGGAAACGG 301 TAGCACTCAG TTGGGCTTCA ATCCTTTAAC CCCACCTGGT CTACCCAGTG CAGTCTTACC ACCAATTTCA CATTTCCATC ACGCTATGCA AAGTCAATTG 401 GCAGCCTCCG CCAATAACAC ACCCACTCCA ACTAGTACTC CTCCTATGGA TGTTACCCCA CCGAAGTCCC CAAGTTTCCT GATGGACACC TCTGCTAAAG 501 ACTCAAACAC CGATCACGAA ATGATGTCAA ATTCAAGTGA AGATGGTAAG GATCTCTTAG AAAGTGAAGA CGATGAAGCA ATCAACATGC CAATCTACAA 601 CTCTCATGGT AAAATGAAGA ATTACAAGTG CAAAAGCTGT GGTTTCACTG CTATTACAAA AGTGTCTTTC TGGACCCATA TGCGATCTCA CATKAAACCA 701 RAAAAGGTGC TCCAATGCCC AAAATGCCCA TTTGTCRCCG AACTAAAACA CCATTTGGAA TATCACATTC GCAAACACAA GAACATCAAA CCTTTCCAAT 801 GTGATAAGTG CAACTATAGT TGTGTAAACA AGTCCATGCT GAACTCACAC AGGAAATCTC ATTCCTCTGT ATA

Source: 5' RACE product, amplified from an embryonic cDNA template. At position 422, a cytidine (C) has been added to the sequence. The sequence of this particular 5' RACE product lacks this cytidine, resulting in a frameshift and a premature stop of the ORF compared to the sequences of three independently cloned, though slightly shorter putative zygotic 5' RACE products. The additional cytidine was also found in the genomic clone (see SEQ09, 2300). Therefore, the cytidine has been included in this sequence.

\section{SEQ09 Megaselia hunchback, genomic.}

1 AAATTATGAA ATTTGAAAGA ATTCAGTTAT TAAAACTAAA GAAACTACGT TTTTATAATG AACTAAGAAA AATTGTTATG ACATAAATTG TAAATTTTTA 101 AAATAAAAAT GTTGGCTGTA AAATATTTCT TAGTTTAATT AAATTTTGTT TAACTAAATA TTATTAATGA TAATAATTTG CTCAAATTTA TTTGGTTTTG 201 ATAATGCTGT CTTAACTATG TTCATATATT TGTCAAATTC TTCAAGTCTT TATAAAAGTT TACGGATGGA CAAAATGTTG TATTGTAAAA TAATGAAGAT 301 TCCCAACAAA ACCATCTTTC ACTTTTTGTT TTTTCACTTT CAATAAATAA TGTTGCGTTA AAAGAAGAAC CCCTTAAGGT CATTCAAATT GACCGAGAAG 401 ACCATGTTGA TGGCCGAACA TAACTAGAGG TCAAGGCGAA GAAAACAAAT CCATCGTCTT TAATGAAAAC AAGAAACCGA AACCGAAGGA AATATAAATT 501 CGTGTGAATT ATTCAAATCA TCTTGCAAAT ATAAACAAAT AGTGATTAAA TGCAATAAAA ACCAACACTA CTACAAAACT ACCTACCCTA TCTACCGACC 601 CTCCACCACA TCAATGTATG TGTGTCACAA GGACCACAAA TCTCTCTCTC TCTCTATTTT GTCTAGCCGA AGATTGCTTT GCCTGCCTCT CCTACAGCCA 701 TGTAATGAGA GAATCTTTCA ATGTATCTGT GTAAAAACTA TATGAGAAAA AAATTGGAAA AATGTATATA ATTTTTTGTA AAGTGATTTT TTTGTACAAA 801 ATCAAATCAG TCGTGCTCGC AACTTAACAC GAGAAAGGCA AAGGATGTTA GCTGCTCGTG CCGTGTAGAT CACGAAATTA TTACAATATT TTTTACAAAA 901 AGAAAAAAAC AGTGACAAAA ATTTTAAGAA TTCAATAAAC AAAAAATAAA AAGAGAAAAA AACAAATAAA GTGACAAAAA ATCAACCGTT ATTTCCAAAA 1001 AAATTGGATT TAAATTTACG AACAATTTTC GCTAAAAAGG TAAGAATGGA TTAACTTTTA AGCCAAATGG ATTAGTTATT ACTGTTTCAT ATAAGTAGTT 1101 AGGAAAGGTT GTAGAACCAA GTCAGTTGAA GCAGAGAAAT CGAAGAGATA GGTAAGCGAA TAAGTCAGTC CGTCTGTCTG TCTAATCAGC TGATGCACAG 1201 ATAAAGTGTT GAAATTGATA GACCGTCATC CTTCAAAAAG ACAAAGAAGA ATTTTTTTCA AGAATAAAAA TTATTTCAAA GTCAAAGAAC ACGCAAAAAA 1301 TAACAAAACA AAAATATTAT TTAGAAAAAC TTGGCAAAAC AGTTTTTCTT TTTTCATTTT TCAATTTTCA TTGCATTTGA CTATGCAAAA GAATATGACG 1401 AACAGTTTTC TTTCTTTCTC ATTTTCATTT TTTTTTGTTT AAAAAATAAA TTTCTAATAA AAATAGAAAA ATGTATATTT TTCTTTGCCG TTTTTCTTTT 1501 TTGTTCGCCA ATTTTCTTTA TATGCACTTT ATAGTGCATT TTGCTGCTGC ATCTGTTTAT CTCGGCATTC AGGCAAACAA CAATTTTTAT TTTATTTTCT 1601 TTTTTTTTTG TTTCTTGCTT TGCACATTTT TATGAAAATT TATAGTTTTT ATTTATAGTA GTCGGGCATG GTGTGTATTG TATATACGTA TAGTTTTTGA 1701 ATAGTCCCAA GAACTTGACC GGAAAGCGGG CGGGCGTCTA GATATTACGC AGTGCATTTC AATTTTTTCA TAGACTTTTT GTTTTTGATA ACCTTGAATA 1801 GTTTATTAGA AATTCTAAAT AAAAAGGATA AGGGATGTTT GAGTTTACGG ACACGCGTAC AACTTGTGCC TGAAGAAAAC AGAAATCAAA ATGGCGTTTT 1901 ACTAAAAATC TCTTTCTTCC TTACAGATAT ACAACAAAAA TCAAAATGCA GAATTGGGAA TCATTACAAC AAACAGCTTC GTATGAGCAT AATTGGTACG 2001 GAAATATGTT TCCAGCCACA CAAATCAAAA CAGAGCCTCT GGAGCCATCC AGTCAACCAT CGCAATTGGA ACAGTATCTC ACATCGATGA AACAACAACA 2101 GCAACACACC AACGAAATGA ATTCAATGAC TCCATCACCA AGAGGTGAGA ACGAAACACA AAGTTTCTTC GGAAACGGTA GCACTCAGTT GGGCTTCAAT 2201 CCTTTAACCC CACCTGGTCT ACCCAGTGCA GTCTTACCAC CAATTTCACA TTTCCATCAC GCTATGCAAA GTCAATTGGC AGCCTCCGCC AATAACACAC 2301 CCACTCCAAC TAGTACTCCT CCTATGGATG TTACCCCACC GAAGTCCCCA AGTTTCCTGA TGGACACCTC TGCTAAAGAC TCAAACACCG ATCACGAAAT 2401 GATGTCAAAT TCAAGTGAAG ATGGTAAGGA TCTCTTAGAA AGTGAAGACG ATGAAGCAAT CAACATGCCA ATCTACAACT CTCATGGTAA AATGAAGAAT 2501 TACAAGTGCA AAAGCTGTGG TTTCACTGCT ATTACAAAAG TGTCTTTCTG GACCCATATG CGATCTCACA TGAAACCAGA AAAGGTGCTC CAATGCCCAA 2601 AATGCCCATT TGTCACCGAA CTAAAACACC ATTTGGAATA TCACATTCGC AAACACAAGA ACATCAAACC TTTCCAATGT GATAAGTGCA ACTATAGTTG 2701 TGTAAACAAG TCCATGCTGA ACTCACACAG GAAATCTCAT TCCTCTGTAT ACCAATACCG TTGTGCTGAT TGTGATTACG CCACTAAGTA TTGCCATTCA 2801 TTCAAATTGC ATCTCAGAAA GTATGACCAC AAACCAGGCA TGGTTTTAGA TGAAGAGGGT ATCCCAAACC CATCAATCGT TATTGATGTT TACGGAACCC 2901 GCCGTGGCCC AAAGATGAAG GGAGGTATAA GCACACCATC AGTTTCCCAT AGGAGAATTG TGCCTGATCA AAAACCAAGT TTGTCCGATT TGAAAATACC 3001 CTTCTCACAT TTGCCAACCT CCCCCGCTAA AAGTACAACT TCATCCAACT CGGAATACAA CACCCCACCA AGTTCCGCCA ATCAAATGAA GCCCAATGGA 3101 CAAATCTCAA ACCTCCTCCC ACCTTTGGTT CAGAGTATGC TTCAGCAACA ACAACAAATG AGCGGTTTCT TCCCCTACTG GAACTTGAAC CTCCAAATGC 3201 TTGCTGCCCA ACAACAACTA GCTCAATTGT CGCCAAGTAT GAGAGAAAGT CTTCAACATC AACAACAACG TTTTGACAAG GATTCCAGCC GCGAATTCGA 3301 TGTTTACGAA GACGAGGAGG AAGAAGACGA ACACGACCAG AAAGAAGAGC ATGTCGCTGC TGCCATCGAT TTATCCGCCC AAGCTTCTAC ACCTATCAAA 3401 GACGAAGAAG AAGCTAAGGA GGAAGAAACC AGCAGTAACA CTCCCACCGT CAGCACAACC TTCATTTCAA GAAGGAAAGG ACGTGTCCTC AAACTAGATA 3501 CTACAACCAA CACTCAAAGT CAAGTTGATG AAGACTCTGA CCGTCAATCC CCATCCTCTT CCTTTGAAGA ACCAAAAGAA ACAGCTGCCA CGTCGACCCC 3601 TAGTCCAGCC CCAGCCCCAG CATCTCCACC CAGTTCAAAC CTCTTCGAGT GCAAATATTG TGATATTTTC TTCAAGGACG CTGTCCTCTA CACCATTCAC 3701 ATGGGCTACC ACAGTTGTGA TGATGTATTC AAGTGCAACA TGTGCGGGGA GAAGTGCGAA GGACCCGTCG GACTATTTGT GCATATGGCA AGAAATGCTC 3801 ACTCGTAAAC TTCTCTTAAA GATAAGTTTT CTGAAAACGT TAATTTTGTA AAAAAAAAAA ACAAAAAAAA ATAGTTTGTA AGGCCAAAAA CTGTTGTCAA 3901 AGATTGTACA TATGAATTTA GACTGTAAGT GTTTTTTCTT TTAATTTAAG CTATAGTATT TTTTAAGAAA CGCAAAACAA ACAAACCAAA AATTGTCTTA 4001 ATTTTAATTA AAAAACTTTT TTAATATTGT AAAAATTACA AAATATTTTT TCCTTATTTC TAAAAAATAT AATTTTTCCT TGCGTGTAAA ATAATTTATT 
4101 TCGTTTTGAA AAGAAATTTT CTAGTCAATT TTTAGTTTTT TAGAATAAAT TTTTATTTAg TTTAAAGAAA ATATACTCAA CAAATTCGAC AATACATAAA 4201 TGGTACAAAA CTGATGAAAT GTACAGTAGA ATTAAAGAAG AAATGGGTGG TCGAGGCTTT TCGAGCGTCA CGAATCAAAA TGAATACTTT TTATTTTTAA 4301 GCTAATTTTA TTTTTCATTT TTTACTAAGA ATAAAATGTT ATTTTTCAAA ATTATTTCAA TTGTTTTTTG GGGGGTATAT ATTTTTTTTT AGGTTTTTAG 4401 GGGTTTTTTT TAAATATTGT TATATTTCGA GAAAAAATTT TGAGATATAT TTTTAAAATT TTAAAATTTC ATTAAAATTA GGTTTTGATT AAAAAATCCA 4501 CAAAAAAAAA ATGGATTTAA TAGATACAAC TAATTTTTAA AATTTAAAGC TTGAAAAAAA AATATTTGTA AAAAAGTTTT GAAATTGAAT CTTGATTAAA 4601 GTTATAGATT TAAAGTTTAT TTATTTATAA CCTTGAATAT GTTATTAATT TTTAGGAATT TTTCTAAAAA ATATATATTT TTTTTATAAA AAAAACAGAT 4701 ATAGAACAGA TAAAATAAAT TCGAACTTGA TTAAAGTTGC AGGATAGGTT CAATGAAGTC TGGAATAGCA GATAGGCTTT TTTCGTAATG TTACGTTACG 4801 GTTAATGTTA CGGTTTTGAT GAAATTACGT AACGTAGTTA AGATTTTCAT AGACTTCTAA GTTTTTTCCA AGTAATTCTG GGGTTGAAGG CCAGGGAAA

Source: Genomic DNA of phage Mab-hb ph2a, which was partially sequenced in a subcloned SpeI (1..2309) and an $\mathrm{XbaI}$ (1738..4899) fragment. Alignment with cDNA sequences: Positions $823 . .1039$ correspond to the first exon of the Megaselia hunchback P1 transcript (Stauber et al., 2000), and positions 1104..1151 correspond to the first exon of the P2 transcript (SEQ07). Positions 1927.. 3861 are presumably common to the second exon of both Megaselia hunchback transcripts. Three putative polyadenylation signals were identified in the genomic sequence $(4144 . .4149,4330 . .4336,4714 . .4719)$ and one putative NRE sequence (3891..3912). Bicoid-binding sites identified by DNaseI footprinting are underlined (Philip Shaw, supplemental Figure S3; 1041..1044, 1047..1059, 1063..1073).

\section{SEQ10 Platypeza hunchback, cDNA, P1 transcript.}

1 AGTTTAGTTC AGATTTGTGT TTCTGTGAAT ATTTATTTAT TCGTTTTGTT TGTGCGGTTG GACAAGTTTT CTTTTTCAAA GATCAAAATG CGATTTGTTT 101 TTGCTTTAGA TCTCTAACAA AATGCAAAAT TGGGACGCAC TTCAGCCAGC TAGCTACGAG CACAATTGGT ACAGCAACAT GTTCCAAAAT ATTAAACAAG 201 AGCCGCAGA CCAACCCACC TCCCAACTGG AGCAATATCT TACAATGAAA TCGCAACAGC ATCAGCAACA CCAGCAACAA CAACAACAGC AACAGCATCA 301 TCATCATCAT CAACAACAGC AATCGCAAAA CGTTGATATG AATTCGCTAA CACCTTCGCC GAGAGCGGAT AACACAGATG GACAAAGTTT CTTCGATCAT 401 ATGCCACATC CGCTAAGCGG TTTCAATCCG CTAACCCCAC CGGGTCTGCC GAATGCCGTC TTGCCGTCCA TGTCGCACTT CCTTGCCACC AGTCCGCCAG 501 AGAATTTGAA TGCGCAAACG CAATCGCTGA CGCCACGCAA CACACCGCCA ATGGATGTGA CGCCGCCAAA ATCGCCGAAA CCGGAGTTTT CCATGTTTAT 601 GGATAAAGA CAAGATTTGA TTTCCAACTC CAGCGATGAT ACGAAATTTT TGGAAAGCGA AGACGACGAG AACATTCGGA TGCCGATTTA CAATTCGCAT 701 GGCAAAATGA AGAGCTACAA ATGCAAGAGC TGCGGATTAA CAGCTATTAC GAAAATCGGC TTCTGGCAGC ACGCTCGCAC TCATATGAAG CCCGAGAAGA 801 TCTTGCAGTG CCCCAAGTGC CCATTTGTCA CCGAGTTGAA GCACCACTTG GAGTACCACA TCCGCAAGCA CAAGAACTTG AAGCCATTCC AATGCGACAA 901 GTGCAGTTAC AGCTGCGTCA ACAAATCGAT GCTCAACTCG CATCGCAAGT CCCATTCATC GGTCTACCAG TACCGTTGCT CCGATTGCGA TTACGCAACG 1001 AAATACTGCC ATTCGTTCAA GTTGCATTTG CGCAAGTATG ACCACAAGCC TGGCATGGTT TTGGATGAGG AGGGTCTGCC CAATCCCTCG ATTGTCATCG 1101 ATGTGTACGG TACACGTCGT GGCCCGAAAg TGAAGAACGC CAACAACAAG GCAAACAAAg CGGCTGCCAT CAAGTCTGAA ATGAAAATTC CACAACATCC 1201 CAACCATCAT CAGCTGCCAG CCTCGCCTGC CAAGAGCACC ACATCTTCAT CGTCTGACCA CCCCAACCAA CAACAAATGT CGCAACAAAC GCCCCAAATG 1301 GCATTGGCAT CGATCCTCCA ACAAGGCCAC AACATGCCCT CATTCTTCCC CTACTGGAAT CTCAATCTGC AAATGTTGGC TGCCCAGCAA CAAGTGTTGG 1401 CGCAAATGTC GCCACGTATG CGGGAAGCCA CCCTCCAAAA TTTGCATGGC GGACAAAGCA ACGACGACAA TAACGCTGAA GACAACCACA GCTTCGAGGA 1501 TGAGGACAAC TTTGACCAAA AATCGGAAGG CAGCGCAATG GACTTGTCCC AAGGCTCCCC ATTGAAAAAC GAATCGTCAC CCCCTGTCCT ACCATACAAC 1601 CTATTGAAAA TGACCGAAGA GGAAGTCAAC ACCCCCACAA TCAGTTCGTC AAGCAGCTCC CGGCGAAAGG GCCGTGTCCT CAAGCTAGAC ACATCCACCC 1701 AACATCTCCC AGTCGCCGAG GAAATCGCCG TGCCCGAGCC AATCCGCAGC ACTGAGTCCC CCTCGTCGTC GTCTTTCGAA GAGCCAAAAA TGGTGCAATC 1801 CCCCGCACCG GTCGCAGCAC CAGTTGCTGT TGCCCCAATC ACCACACCCT CCCCGCCCGC CGTAGTCCCG TCGAACAGTA ACATCTTCGA GTGCAAGTAC 1901 TGTGATATTT ACTTCAAAGA CGCGGTCCTC TACACCATCC ACATGGGCTA CCACAGTTGC GACGATGTGT TCAAGTGCAA CATGTGCGGG GAGAAATGCG 2001 ACAGCCCTGT CGGACTCTTC GTCCACATGG CCCGCAATCC ACACTCATAA TTCTATTACC CGTTATTTGT TTTATTATTA TTTGTTGTCA AAGATTGTAC 2101 ATACGA

Source: 5' RACE product (1..893), 3' RACE product (1457..2103) and an additional PCR product (864..1704), all amplified from a larval cDNA template. At positions 301..303, an additional TCA repeat has been added to the sequence. The sequence of this particular 5' RACE product lacks this repeat compared with two independent, though shorter 5' RACE products. The additional TCA repeat was also found in the genomic clone (see SEQ11, 6594..6596). Therefore, the TCA repeat has been included in this sequence.

\section{SEQ11 Platypeza hunchback, cDNA, partial P2 transcript.}

1 AGTCGTGTCT GTAACCGTAC AAGAAGAAGG ATGTGTACTG CCGTCGTGTA GATCACGTGA ACAAAAAAAG AAAATAAATA TTATAATTTT TAATTGAAAT 101 TTTAAAAATT CAAAAAAAAA AGAAAAGCGA TTTTGTGTGT TTTTAATAAC TGTTATGTGC TAAACGAGTG AAATATACAA ACAAATATTA TTATAAATTT 201 TTTCCCACAC AAACTAACGG ATTATTTGAT AGAGCAATTT ATTTGCTTTT CCAGAGAACG AGTTAATTGA GATCTCTAAC AAAATGCAAA ATTGGGACGC 301 ACTTCAGCCA GCTAGCTACG AGCACAATTG GTACAGCAAC ATGTTCCAAA ATATTAAACA AGAGCCGCAG AGCCAACCCA CCTCCCAACT GGAGCAATAT 401 CTTACAATGA AATCGCAACA GTATCAGCAA CACCAGCAAC AACAACAACA GCAACAGCAT CATCATCATC ATCAACAACA GCAATC

Source: 5' RACE product, amplified from a larval cDNA template.

\section{SEQ12 Platypeza hunchback, genomic.}

1 CTGATTCGAG TTATGACATC CAAATCAGTT CCACCGGGTG CGGCGATTTC GCTGCCTAGg TATGTGAACC TTTCCACCGT CACAATTTTG TTGTCCAGCG 101 ATTCGGGTTT CCTTCTAGGC TTAAAACCTT AGTCTTTTTA AAGTTGATCT TTAACCGGAA GACTTCTTCT CCTCAAACTC TAGCGATTGG GCCACGTTGG 201 ATAAATCGAA TATCCGGTGT GCTATGGAGG CATATGTCGT CGGCATAATC CAGGTGTTGC ATCAGGAAGC TACATATAGT TTATATAAAT AATAATTTTT 301 GTAATAAAAG ACGAAATTTC AGTTAGCTTC TCAGATATTT AAATTAAAAT TTTATTTAAA AAAAAGAAAG AATAAAGTTA AAGTAAGCAA ACTGTTTAGT 401 TAATCTCAGT TAATAAGCAT CGATATAATG AGTTCAGGTG CTTGATTGAT ATTCAATTTG AAATGTTATT CCGGGATTTG TTCAAATTGG CTTATTTCTG 501 TTTTCTCTTT TTTCCACTCT AAGACTCTCT TGGTGTCCAT ATTAAAATAA ATGATTGTGA AATGTTAATC ATTTGTTCTA ATTTTGTATG AGTTACGATT 601 TATAATTTTT TTCCCAAATG TTACATCTTT AAAAAATGTA TATCATAGTT ATTACAAATA TGCATTAACA ATGTAAATTA ACGAGTTGCT GAAAAGAGAA 701 CTTTTTAATT ACAATCCTAT GTATATACGA AATAAAGGAA GTCTCAAGTA TTACAAGAAT GATTGATAAA AGAAGATAAA AACAATTCAA CCAAAATATC 801 TAAAACGGAA TCTTCCTCAT TGACTTCTCA TTACACAAGA AAAAAAAAAC GTTTCCTTTC TAGAACAGTA CCTAAATGTG TCCTTAACTC TAATCCGTAC 901 ATCTTAACTA CTTTAATCAC CACACAAATG TCCAGTCCGT CCAGTCGCAT GCAGACCTCA TCAACCATCA CAAAAACAGA CACAATGGAG TTCCAACCTC 
1001 TTTAACACTT TTGTTCTGTT GTCTGTTGTC TGCTGGTCTT TTCCATATCT CTGCTAATCG TCAATGAATA TTCGTTTCTG GGCTTATTAC AAACACATTA 1101 CCATGACTAT GCAAATTTGT TGTGTCGAAG GACCTTCTTT TGGGTGTGTG TACATTCTCT AACCGCATAT GTCGGACGAA TGTGCGATTT TGATTGAAGA 1201 AACCCACAGG AATTTTTGAT TTTTTGTAAAT ATTTACCGAA GCGAACGGCA GAAGGCGCAA TCGGTAACCG CTACTGGATT TGCGATTTTC TTATCTGGGT 1301 CTATTGTTGT TTGAAAGGAG AAAGGAGGTA GATGTAAGGA CATGAGGTCA ACTGATGTGA TGACGTTGCT ATTATTACGA TGGGTCAAGG GGGACGATCC 1401 CCTTACGATG AAAGATTTTA TATATTATGT AAATTAATTA AAAGGAAATT TCTTTAAATG GACATTATTG GGTCAAGGAG TGTTTTGAAA ACAATTGACG 1501 TGCGCCAAGG TCAACTGACT ATTGGGCAAT TCTTGAAAAT AAATAAATAT TTGTCGATTT ATTAATTGTA CATTATCGCA TATCAATAGA AAACAGAAAA 1601 ATACCGCACA TGTGAGGCCC CATGCGCATT CACAGCAGAA AAACAAATCT TTCATATCTT TTTTTTAAAAT CTCAATTGCG ATTAAACGAT TTGCGATTTA 1701 GGCTATGCAA AAAAATTTTTT TTTTTTTGGCT TCAATCATTC ACTTTAAAAT TATCTTTAAT CGCGATTAAG TCCTTTTTGT GTTAGCTTAT ACATAATTAA 1801 ACCGGCAACT GGCGCAACTG CGTTGAATGT TCGTGGTTAA ACTTTATTAG TTGTTTCTTT CTTGTGTGGT TAAAAAATCA TTGTGTGTGT CTGGGTCACT 1901 GCTACAAAAA TATATACATA TATTTATTTT GCTTGACTTT GCTTATTTTG AATACCTACC GATAATACAT AGACTGCTGA GAGAGCATCA AAAATCACAA 2001 AGAAAAGTGT AATAAAAATG ATTTAAAAAAT TTTTAAGCTT AAGAATAAAT ATAGTAAATC TCCCAATGAT ATGATCCACA TATAAAACGC TTAAAAATAT 2101 TAAAAACTAA TATCGGCATT TTTACTAACA AATTATAAAA TTATGGGGGA AAGACATTTA AAATATATTT TTTATTCATA TACCTAAAAT TTTGTTTTTC 2201 TTCATTTTTT AGCAAACATC AGAGTAATAT TGGAAATTGT ATATTACCAT TTTTTTTTAAA ATATACCACG AACCATATAA CGAACCAAAA AATATAAGAT 2301 TAGATTTAAA ACTAACTGCA TCAGTGATTA AATGGCTAAT TATTATATAG TATTAACTAC AGCCCAGGAC CCCGATTAGA TAGAGGAACT CGTAAGACGA 2401 ATTTAAAAAA AAGAAACTAA GCACATGTCC TTCTATTAGT AGCTTTGGGA CAAAATAGAT GTTTGATCCG AATCGGCGGA AAAGCAGATT TATGTATAAT 2501 TTAAAATTGG ATAGCTCTTA TATTTAAATT TAAAAAAAAA TCCGCTTATT ATAAATAAAT TATTAGATTT TTTTTTAAAT TTAAATATAA GAGCTATGTT 2601 TAACTAAATA TATATTTTAA TAAAGACGTA ATTGACTGTT TGTTGTGTTA TGCCAAAATA TATAAAATAA GTAAAAGAGT CTGTGAGAAA ACGACAGCAG 2701 TCGATATTCC AGCTCATATG AGTTCCTGGT ACAAACGAGA TTCTTTTTCC TTCATTTTTT TTCTTCAATA ACAATCAGTC AGTAAAACGA TTCCTTGAAA 2801 TAAAAATGTA TAAAAAACAT ACATCGAAGG ATGTGTTTAC ATCACATTGA TGGAGCAGAC TAGACGGCTG TGTGTGGATT AATGGTATGG GGAGATAAAT 2901 GAAAGCAATA AGCTTCGCCA TTTTTTTTTA TTAATCGAGG GGTTTTATTT AAAATAAACG ATTTGAAATT CTTCGAAGTC AACATATGAA CGTAGGCAAA 3001 ATCGTGAAAA ATATGACAAT TTACCGTTAT ATAAATATTT TATAGTAATG GTTTTTTATA TTTTAGAATT TAGAAGAACA AAAAAACATT TAAAAATGTT 3101 AAATGTTAAA AAATATATAT TTTATATAAT AGTTTTTTTTT TGTTGAGATA ATAGAATAAA CAAAATTTTTT ATAAGAACAA AAATAAATAA AATATTTTTAA 3201 AAATCAAGGA AAATATTCAA TATTTAAACG TATAAAAAAT ATATCCCTAC AATAAATTTT TAAAAATAAT AATAATACAA TGAAAAAATA TTACTATTTT 3301 TTTAGGAGAT TTTTTTCCAT ATTTTTTTAAA AGAAATAAAT TATATGCAGT CTGGGCAACT AATTAAGAAT ATAGTTTTCG ATTAACAGCA AATATATATT 3401 AAGCGATTTT TTTACTAATT CATTTTTTAAT CAGCTGACCA TGACTGGTAT ATATATTCAA AATATATTTG TTCTTATAAA GTATTCATAT AACGGTATTG 3501 TATTGTTACT AAATAACATC AATATTTAAA TTATATATTT TATTTTTAAT GTAAATAATA TAATAATAAT TTTTTAAAAA CATTAAAACT TTGTAATTGT 3601 ATCGGTATTT TTAATTTATA AAAATTACCA GTAAAACATA CAAAATATAA AATTCTAAGT AAACAGAAAA ACGGATAAAA AATATACCTA TAAATAAAGG 3701 ATTAAAATTG AAAAATATGG AAAGGTCTGT TGACGGATTG GCAGACAGAT AATACAATTT TAATAACCCC ATCGACTACC TTTTTGAAAA TTTAATGACC 3801 ATAGTTAGAA ACCTGCCGAT TATTTAATCA GAGCAATATT CCTAATCTGG GTACAAATAT AAATATTAGA TTACAAACAG TTTAGAAGGC AGTCAAATCG 3901 CATTCAGGCA GAAAGGTCAA CGGATAAAAA TAAAATATAC AATTTGACAG AAACAGAGGA AAGGATAAAA ATATATCCCC TTTTTTGTTT ATATTATTTT 4001 CGATGATGTC TACCTATCAC TATATATTTG CTAGCCAGCA TCGTTAAGGA TATGTAAAAA GGATATATGT AGGTATAACG TCATTCATTC AAAAAATCAA 4101 CAAAAATCCC AAAAGAAAAT AAACCCAAAT CGATAACCGA AATGAATTCC GTTCATCTAT ACACAAACAC ATGGAAAACC CACAAAAAAA ATATAATAAA 4201 ATAATAAAAT AAAAAGAAAC AGAAAGTACA ATTTCCTTGT AACAACGAAC GGGCATCCCT TTCTGCAGCA TACAAACCGG CAACTGGCCA TCTCTGTCCC 4301 AACCGTCTGC AGCCGATAAT GTACGGGAAA ACTGTCATGT AATTTTTTCGA CGAGATGAGA ATTTTCGGTT TTTGAAATCA GTCGTGTCTG TAACCGTACA 4401 AGAAGAAGGA TGTGTACTGC TGTCGTGTAG ATCACGTGAA CAAAAAAAGA AAATAAATAT TATAATTTTT AATTGAAATT TTTAAAATTC AAAAAAAAAA 4501 GAAAAGCGAT TTTGTGTGTT TTTAATAACT GTTATGTGCT AAACGAGTGA AATATACAAA CAAATATTAT TATAAATTTT TTCCCACACA AACTAACGGA 4601 TTATTTGATA GAGCAATTTA TTTGCTTTTC CAGAGAACGA GTTAATTGAG GTAAGCATAT ATTTTTAATT TTTTTTTTAA TTCCAATACG ATCAGGTCAC 4701 CСTGCTTATT TTTCCTTCCT TACCTTTACT TAAACAAATC CTTAACATCA AACCTGCAAA TATCCCTAAA ACGTACCTGC CCGTCCTAAA ACGAGTTGAT 4801 TAAAAATCAA CAAAAACAAT TTCGAAAAAA TCGTTGGATT GATTGACTTA GTCTGAGATA ACCCCCGGCC ATCAGACATG TCCTCATTTT GTTGCAAATC 4901 CGCGTATCAA TTCGGGATTA GGCGCTGGGA CAGATACCTG TCCAAAGTGC TATATATTCC GCCGATATTT TGATTTTGAT ATCAGTCGTG AAATAGTATT 5001 CCATCATAGT TGAGTGTGTC TTGCAGACAA TTATTGAATT CATTTAATTT TTATTATTAT TTAATATCCG TTTTTGGTGA GTAATTTATT AATAATTAGT 5101 TTGTTTGGAT ATTTTTAATA TTTACAAAAC AATCCAAGGC CAAGTGCATT GAAAAATTTC CTCAATCCTA TAACTAAAAT ATATTTACAT ACATATAAAA 5201 CCAGATGCAC AGAAAAACAC AAAATCGTGG AAAAAAAAACA TTCGGTCGAT AAATATCAAA AATACACCCA CCCCCCCACT TTTACATTTC ATATATTTTG 5301 TATATACAAA AATATACCCC CTTTGTCCAA ACCGTACAAA CCGTCCAAAC GGTATTTCGA GCACACACGC GCATTTTCTA TATTAATAAA AATAAAAAAA 5401 TGCATAAATA AAACTATTTT CGCGAAAAAG CAAAAACGAA GATCGTTCCG GTCAAGTTCT TGGTTITTTTT CCTCACTCGT TTATTTAATT TTTTTGTTTGT 5501 TGCACTCATA TGAGCATCAG ATATTTTGTG CATTAGACAC ATTATTTTAT TTATATACAT ATTCATTTTT CGATATTTTT AAAAAATGAT GCATGTGCAA 5601 AATAGTGCAA TCTCAGTGCA ACAACAATGC ACACAAAAAT ATCAACAACA CAAAAAAAAA ATATTTAGAA AACATTTGCC AAGTTTTTTTA GTGACTTTGA 5701 ATAATTGGCC TGTGTCTATA TTTGTAATTT TCAAGTATTT TAAAAATTTA AAAAAAAATT AACAAAAAAA GAAAGGATTT GCGATAGTAA TCCATGTTCA 5801 GGTATATATG CAAATTATGA AAGTAAATAA AAGGTTGTTT TTGCGAAATT TGGGTAATAA AAGAATTACT CCAGATTTGG TTTGACTTTT TCCAAAAGGC 5901 AAGGACTGGT CATTTATAAC GGCTTAGTCC TTCTTAATCA GTGCTTGGTA AGGTAGAAAT TACCTAAAAA CAGGCACAAC GCCATTTTCT TTATATACAC 6001 ATTTTTTGAAC ACGAGTCAAA ACTACTAAAA CAGTTCCCCC TCTTGGATAT TACTCATAGA CACATAGGGT GAGTTGAAGT TGAGATTTAA ATCCAAAAAT 6101 CGCCTAAAAA TACGCTTATG TACTGTAAGC GTTATTAAAA AAGGGATGGT CTTATGTATA CACCCCGATG TGTTGCTAAT GTAAAAATAC GTAAAAATAC 6201 CCTTAGGGGA GATGTGCTAT TTATGTATTA TTGTACATTT TAGGGGTTGG ATATAATGGA GGAAATTACT TGGGCACGCG CATTGCTTGT GCCAATTTTT 6301 TATTCGTTTT TTTATTGGTT TTTTTTTTAT TTGATTTTTA TTAGTATATA AAACGTTTTA AAATGGCGGA CTTTTATTTA TAAATTTTTT TTTAATTTCC 6401 AGATCTCTAA CAAAATGCAA AATTGGGACG CACTTCAGCC AGCTAGCTAC GAGCACAATT GGTACAGCAA CATGTTCCAA AATATTAAAC AAGAGCCGCA 6501 GAGCCAACCC ACCTCCCAAC TGGAGCAATA TCTTACAATG AAATCGCAAC AGCATCAGCA ACACCAGCAA CAACAACAAC AGCAACAGCA TCATCATCAT 6601 CATCAACAAC AGCAATCGCA AAACGTTGAT ATGAATTCGC TAACACCTTC GCCGAGAGCG GATAACACAG ATGGACAAAG TTTCTTCGAT CATATGCCAC 6701 ATCCGCTAAG CGGTTTCAAT CCGCTAACCC CACCGGGTCT GCCGAATGCC GTCTTGCCGT CCATGTCGCA CTTCCTTGCC ACCAGTCCGC CAGAGAATTT 6801 GAATGCGCAA ACGCAATCGC TGACGCCACG CAACACACCG CCAATGGATG TGACGCCGCC AAAATCGCCG AAACCGGAGT TTTCCATGTT TATGGATAAA 6901 GAGCAAGATT TGATTTCCAA CTCCAGCGAT GATACGAAAT TTTTGGAAAG CGAAGACGAC GAGAACATTC GGATGCCGAT TTACAATTCG CATGGCAAAA 7001 TGAAGAACTA CAAATGCAAG AGCTGCGGAT TAACAGCTAT TACGAAAATC GGCTTCTGGC AGCACGCTCG CACTCATATG AAGCCCGAGA AGATCTTGCA 7101 GTGCCCCAAG TGCCCATTTG TCACCGAGTT GAAGCACCAC TTGGAGTACC ACATCCGCAA GCACAAGAAC TTGAAGCCAT TCCAATGCGA CAAGTGCAGT 7201 TACAGCTGCG TCAACAAATC GATGCTCAAC TCGCATCGCA AGTCCCATTC ATCGGTCTAC CAGTACCGTT GCTCCGATTG CGATTACGCA ACGAAATACT 7301 GCCATTCGTT CAAGTTGCAT TTGCGCAAGT ATGACCACAA GCCTGGCATG GTTTTGGATG AGGAGGGTCT GCCCAATCCC TCGATTGTCA TCGATGTGTA 7401 CGGTACACGT CGTGGCCCGA AAGTGAAGAA CGCCAACAAC AAGGCAAACA AAGCGGCTGC CATCAAGTCT GAAATGAAAA TTCCACAACA TCCCAACCAT 7501 CATCAGCTGC CAGCCTCGCC TGCCAAGAGC ACCACATCTT CATCGTCTGA CCACCCCAAC CAACAACAAA TGTCGCAACA AATGCCCCAA ATGGCATTGG 7601 CATCGATCCT CCAACAAGGC CACAACATGC CCTCATTCTT CCCCTACTGG AATCTCAATC TGCAAATGTT GGCTGCCCAG CAACAAGTGT TGGCGCAAAT 7701 GTCGCCACGT ATGCGGGAAG CCACCCTCCA AAATTTGCAT GGCGGACAAA GCAACGACGA CAATAACGCT GAAGACAACC ACAGCTTCGA GGATGAGGAC 7801 AACTTTGACC AAAAATCGGA AGGCAGCGCA ATGGACTTGT CCCAAGGCTC CCCACTGAAA AACGAATCGT CACCCCCTGT CCTACCATAC AACCTATTGA 7901 AAATGACCGA AGAGGAAGTC AACACCCCCA CAATCAGTTC GTCAAGCAGC TCCCGGCGAA AGGGCCGTGT CCTCAAGCTA GACACATCCA CCCAACATCT 8001 CCCAGTCGCC GAGGAAATCG CCGTGCCCGA GCCAATCCGC AGCACTGAGT CCCCCTCGTC GTCGTCTTTC GAAGAGCCAA AAATGGTGCA ATCCCCCGCA 8101 CCGGTCGCAG CACCAGTTGC TGTTGCCCCA ATCACCACAC CCTCCCCGCC CGCCGTAGTC CCGTCGAACA GTAACATCTT CGAGTGCAAG TACTGTGATA 8201 TTTACTTCAA AGACGCGGTC CTCTACACCA TCCACATGGG CTACCACAGT TGCGACGATG TGTTCAAGTG CAACATGTGC GGGGAGAAAT GCGACAGCCC 8301 TGTCGGACTC TTCGTCCACA TGGCCCGCAA TCCACACTCA TAATTCTATT ACCCGTTATT TGTTTTAATTA TTATTTGTTG TCAAAGATTG TACATATGAA 8401 AATCACAAAA AAAAAACGAA ACTAAAAATT TCATTTTCTT TTTAGTTTCC TTATAATTTT AAGAAAAGTT GTCAAGGATT GTACATAAAA AAATTAGTTT 8501 TTAAGTTTTA TACATAAGTA AATTTTTTTT TAAAATCAAA CC

Source: Genomic DNA, phage Pco-hb ph1. Alignment with cDNA sequences: The first exon of the Platypeza hunchback P1 transcript (SEQ10) could not be aligned with the genomic sequence, positions $4380 . .4650$ correspond to the first exon of the P2 transcript (SEQ11). Positions 6403..8399 are presumably common to the second 
exon of both Platypeza hunchback transcripts. In the genomic sequence, two NRE sequences were identified $(8375 . .8396,8466 . .8487)$.

\section{SEQ13 Lonchoptera hunchback, cDNA, P1 transcript.}

1 AGTAATAGTT GTTCTCTTGC AGCAGCGAGA GGTCCTGTGT TGTTATTATT TCGTGCCATA TATTTTATTT TATTTTTTTA AATTTGTTAT AATTCGATTA 101 CAATTCAGAC GTTTCATTTT AAAATAAAAA CAAAAAAACA ATTAACAAAT TTACTATTAA CCAAAAGTAT ATTGTTTTCA ATTGAAGTGT ACAAAAACAA 201 TTCATTAAGT TTAAATTAAA ATCACTTTAT CCTTTCACAT AAAAAATATA AAATAGCAAA AAGTGTAATT AAGCATAAAG TAATTAATAT TATGAACATC 301 AACAAATTTT AAAAAAAATT AATCAAATTT GAGGAAAAGA CAATGATGTT GTTATAATAT TTTCGATTAT AGTTACATAT ATTGCTATTA TTTGCTAAAA 401 ATGTTTGTGT AAAATTAAAT AATTGTTAAA CAGTTTTGCT AATATTATAT GTTAAATGAG TTACAAAAAA AAGTGGCCGA CGCGTTCCGA TTAACGGATA 501 TAATAACTAA TACTTATGCT GCTGATATTA ACAACATTTT AGAGACGGAT TAACTAGATC GCTCTCCATC GCAGTTAAAT TTGAAGTGAA TCAAAAAAAA 601 TTTATCAAAA CAGTTGCTAT TGAAACCAAA AACAAATACA AAATGCAAAA TTGGGATACA TTACAACCGA CAGCCAGTTA TGAACATAAC TGGTACGGTA 701 ACATGTTTCC AACAATTAAA ACAGAATCGA TGACTCATTC GCCAACTACA TCACAATTAG AACAATATTT AACAATGAAA CAACAAGAAA TAAATTCGTT 801 AACACCATCA CCGCGTGCTG ATATAAACGG AACTTCAGAC ATTCAAAATT TCTTTGATAG CCACAATCTG CAAAATGGCA GTTTGCATCA TAATCATCCA 901 TTAGGATTTA ATCCATTAAC ACCACCTGGT CTACCAAATG CTGTTCTACC AGCAGTTTCA CATTTCCATC ATAACATTAC AGGAATCCAT GCAAATAATC 1001 CAACGAGTCC ACTGGCACAG CAATCCGAGG GAAATACTCA ATCATTAACA CCACGCAATA CGCCACCTAT GGATATTACT CCGCCAAAGT CACCCAAATT 1101 GTACTCCGAA TATGTTGAAA AGGATCACGA TATGATATCA AATTCAAGTG ACGACACAAA ATTCCTTGAA AGCGATGATG ACTCATCGAT TCGTACACCA 1201 ATTTATAATT CGCATGGCAA GATGAAAAAT TACAAATGTA AAAGTTGTGG ATATATGGCT GTAACAAAAG TAGCATTTTG GGAGCATGCA AGTTCTCATA 1301 TGAAACCCGA AAAAATTCTG CAGTGTCCAA AGTGTCCATT TGTTACCGAA TTAAAACATC ATTTGGAGTA CCACATTCGC AAACACAAAA ATTTGAAGCC 1401 ATTCCAATGC GACAAATGCA ATTATAGCTG TGTCAATAAA TCAATGCTGA ATTCACATCG TAAATCCCAT TCTTCTGTGT ATCAGTATCG TTGTGCCGAT 1501 TGTGATTATG CAACAAAATA TTGTCATTCT TTTAAATTAC ATTTACGAAA ATATGACCAT AAGCCCGGTA TGGTCTTAGA TGAAGATGGT TGCCCGAACC 1601 CTTCGATAAT TATAGATGTT TATGGAACAC GTCGTGGCCC CAAAATGAAG TCGCAATCCG GCGGTGGTGG TGGTAAAATA AGTTCGGGAA CAAAAAAATT 1701 ATCCGCAATT AAAGCTGAAT TAAAAGTTCC ATGTGGTGGC TCTCAACTAT CAGCGGCCTT ACAAGGCCAG CTCCATTTTC CAGCATCTCC AGCTAAAAGT 1801 AGTAATTCAT CATCTTCGGA ATACCCGGCG GTCTCTTCAT CCTCACTCTC ACTAAGTCAG CAAGTTTATA ACCAACAACA AAATCAACAA CAACAGCAAC 1901 AATACCAACA ACAGCAACAG CAACAGCAGC AGCAGCAACA CCAACAACAA CAGCAGCAGA AACAACAACA GTCATTACCA CAAATATCCA ATTTGTTACC 2001 ACCATTAGCC TCAATTCTGC AACAAGGAAG AAATATGTCA TTCTTTCCAT ACTGGAATAT TAATCTACAA ATGCTTGCAG CTCAGCAGCA AGTTCTTGCT 2101 CAAATGTCCC CAAGTATGCG TGAAACTACC ATTCAAAATC TACAGAATGG ACAGTCTCAA GTTATTGAAA ATGATAAAGA TTCCGTCCAG GATTTCGAAT 2201 GCGAGACAGA CGATGAATTT AATCGTCGTT CAAATGGAAG CGCTATAGAC CTGTCACAAT CAAACGGAAC ACCCACCAAA ATCACAAATT TCAATCAAGA 2301 TACATTTCAA ATGTCAACCA ATGTATCAAA TGTGTTAGCC GACAGTGGCA TGCAGCAAAT TAAAAGTAAA GAAGAAATCA ATACTCCAAC AATAAGTTCA 2401 TCATGCAGTT CACGGCGCAA GGGACGTGTT CTTAAACTCG ATATAAATGC AATCAATCGA AATTTAGTAA AAAGTCCCGA AATTCATTCC CATCAATCGC 2501 GATCGACAGA ATCGCCATCG TCTTCATTTT TTGAAGAACC AAAACTGCAA GAAAAATCGC CTCCTCATAA CAGCATGGAT ACCAACAGCA TTTCCATGCT 2601 GAATTCTCCA GAGTCATTAG ATCTTTCATC AACTGCCGTT TCAGCTCCTG TTCCATCAGC ATTTACAACA ACAACCATTA GCAACACTAA TAATAATTCA 2701 ACAACTCCAA ACACTACGAG CACAACTAGT ACCACAAGTA GTAGTAACAT AAGTAATGTT AGTAGTAGTA ATAGAATTAG CAACAATCCA ACCTCCTCTC 2801 AAGGCAACAT TTATGAGTGC AAATATTGCG ATATCTTTTT TAAGGATGCT GTTCTATACA CAATTCATAT GGGCTATCAT AGTTGTGATG ATGTTTTCAA 2901 ATGCAACATG TGTGGTGAAA AATGCGATGG ACCTGTTGGA CTTTTTGTTC ATATGGCCAg AAATCCACAT TCGTAGGCGT TTAATTTTTT TCGCGAATTG 3001 TAACTATATA TATGACTCTA TTCCTAGAAA AGGAAAATAT TTATGGTAAA TGGTTAAAAT AATTTTAAGA GATTAACTCA TCAAATTCTA TTTATTTAAA 3101 AAAAAAAAAA AAATGTTTA

Source: 5' RACE product (1..737), 3' RACE product (580..1308), and an additional PCR product (504..3119), all amplified from cDNA templates of adult females.

\section{SEQ14. Lonchoptera hunchback, genomic.}

1 АСTAATACTT ATGCTGCTGA TATTAACAAC ATTTTAGAGA CGGATTAACT AGGTATGTGT ATTGCAACAA ATAACTGCAA CTTTCGAATA ATTTCAAAAA 101 TAAAAAATAT TTTTCTTGTT TTTTCATAAA TTATCAAACT AATGTAAAGT TAAATCGATT TTTATGAATT TATTTTATGT CAAACATTAA CAATAAGGAT 201 AAAGGACAAA AATACTCTTT CTTATTAGAA TTAAGTAATT TAATTTAATA AATTACGGTT AATGACGTCA TGCTGTAACA CTTCATCTAA AATAATATAT 301 CCCTGAATTA TTGCATTTCA CTAAACCAAC GTCGAATAAT GTATTTGTAA ACTTTTTCTA TCTCCTTTTG ACGAGGTGAT TTATTTGCTG GAAACGGTTT 401 AAAGGATAAA ATGATATCAC AATCAAAATT TTGCTAAATC AACAAATCCC TAATAATAGG TGATTTTTAA TTGTTTGTTC GTACCTGACG GCTACTGAGT 501 GTACTCTTAA CTATTTCCAT CTCATAATTA ATTATTTTTT ACCTGAAATC ATAAATCGTC ATCCATGTAA ATTATCTATT CGGTTAAACA AACGTATGAC 601 CGACCATGGC CATGAAATTC TGAATCAAGT GAAAATCTCA GTTGTGTCTT GAAACCCTTT CGAAAAGCAT CAAATTATTC AAAAGTTTCG GTTTAGTTTT 701 TGTTTTTTTT TTGGTTTTTT TTTTGTGCAT TTTCAAGTCT AAATCTGCTT TTAATTGTGT CAGAATTTTA ATTCAAATTC AAAAGTGAAG GTAATTAATT 801 TAGTTTTCCT TTTTATTTAT TTTGATATTG TTTTTCGGGC ATAAAAAAAT TAACAATTTG TGACAACATT CGAAAATCTA AGAAATATTT TAAAATAGCC 901 AAACCAGAGA ACTGAAAGTC ACATTAAAAA ACTTGCCAAA TCGCATCTGC ATCATCAGCT CATAATAAAT TTTGTATTGT ATATCCATCC ATCCCACAAA 1001 TGAAATTTTG TTATTCTTAT ATTTTGTTAT AAAAATTGTG TTTAACTACT AACATACACG AATAAAAATA TAAACAAATT ATTTCAAAAG GAATTTTCCT 1101 AATAAAACTA AAGTAAATAA ATAATCGCCG TAAAATTTTA AATTTACTAC TTTAAAGTTT GTATTTTATA TATATATATA TTTAAATATA TATATATTCA 1201 ATAAAAAATA AAGTGCAGTA CAGAAAATAA TCTCGTGTAC CCCTATCCTA TCCAATTGCT TTATTCAAAA TGATTAACGT CATTCGGTGT ACGAAAAAAA 1301 TCAAACGAAA TTTAAAAAAA AAAAAAATAT AATAAAAATA AACTTTAAAA AATATATAAT TAATAAAACA TCTTGTGTGC ATCATCTACC AAGAACTTGA 1401 CCTGCACATT TGGCGATCGC ATTTTAAAAT GCATTTACAC AAAAACAAAA ATCTAAATCA CACGATTTTA TTAATGTAAA TTATTTTTGT TTAAACAAAA 1501 CCAAAAAATT GCATATTTTT AGAGAAATAA ATAAAAAATT CTTTATTTTC TTGTTTTGCA TCAATTTTTG CTGCTTAAAG TTTACCCAAT GCAGATCTTT 1601 CTCGTCTATA CTATCTTAAA TCGTACTCGT ATCAACCTTA ACCGTCCGCT CTATACATGA AAATGCAATT ATTTACTAAA AAGAAGAAGA AGAAGAAAAA 1701 AAAAACTTTA TTTTGGTTTT TAAACTTTTA ATAGAACTGT GTTGGGCACA AATGAAAAGA AAACTTAGAA AATTTTAAAA TTTTTCCAAA AAATTATTTT 1801 TTTAACCCCA CACCATAACA AAATCATTTG CTCCGTTTTT ATTTGATGGT GGTGAGTGTA CTCTGTAATT AATCAATCTT AAATATTAAA TCGAAATTTT 1901 TTTCTAACTC TAGGTTTTCA ATAAAAAAAA AAAAAAAACA TAATTGATGC TTATTTTCAT CCCTGCATGT GATGCACTTA ACTAACAGAC AGAACAAAAC 2001 TAATCTAACC ACCTTAACGG CCAATGCATT TCATTCAGTA AACCATCTGC TTTTTTTCAC CAAAACAAAT ACTACATGAT GATGAAAATT TCAGTTTAGT 2101 GCTCCAAAGA AGCATTTCGC ATTTCTGCCA TTTGAAAATC AGATATTGGA AAATCTTCTC CTTTTTTTTT ACATTTTTAT TCAATCATTA GGTTAGTAGA 2201 GCCAACTTAA TGTACTGTTA CGGCTAAAAA GACTCTAATA TATATTACAT ATATTCGCAA CTTTTGTGTA TAGGAGGATA GTTTTTCTAA TTAATATGTG 2301 TTTTGCTATA ATTTTTTATT TATTTAATTT TTATTTTTTT GTTTTTAATT TTTAGTTTAT CTACACTTGG CCTCTAAAAT AATTTCGATG TGTGGATTTT 2401 TTATTAGATT TGTTACTAAA TTCTCATCTT CCTCTAAATT TTTGAATATC TTGGTTGCAT ATATGCGATG CAGTCAGAAT TCATGTAATG TATGTATGTA 2501 CTTGGTTGTG GCATGAGGAT GGGCGATTAT ATATCAAAAT TCATGCATTC ATGCATCGTT ATTTCCGAAA ATAGAATTTT TGTTTGTTTT TATCGATTAT 2601 TTCATTTTTG CAAGAGTCCT CAAATCGCCT TTCCTAATGG TGTTTAATAA AGGTGCCGTT TTTTTAAATT TTTTTTTATT TTTTTTGGAA AAAACCAAAA 2701 AAAATTTCAC TATCGTTTTA TTTAAGATCC TACAGGACTT TGTTTAAAAT AGTTTCACTT TAATCCTATC GACTTGTAAA TGATTAACAG TTCTTTTACA 2801 TTTGCGAATT CAATTTGACA AATACAAGTT AAAAGTGAAA GTAGATTAAG ACAATACTAC CTGGGTAGTA ATTAAGTTTC CCATCATGAC AAGCCATTAA 2901 CCCATTTAAG GAAAAAAAAT AAAAAAAAAA AATTATTAAA CAGTTGAATA CGAGTTTTAT CACTGTGAAT GTTTGTTTTT ATAACATTGC ATTTGGAATA 3001 TGTAAATATT TCTTTTGTTC TTCTGAAAAT TCTAAAGTAA CAAATAACAA TCCGAAGAAG GAAAATTAAC GGTTGCTTGT TTATAAATTA CCTGGTCCTT 3101 TTGTTTCTGT ATATATCTAT AATATTTTTA CAGTAATACA ACAGTGAACA GTCAAAGTGG TTTGACTTTT CTGATGTTTG ACCATCCCGA CTCCCTCAAG 3201 TTTTATTAGT TATTTCAATT TTTGCAAATT TCTTTTTTAT ACCTATATTA TTGTCTTTTA AATCTGTCCA TGTACAGTAC AGGGCGTTAG ATTAGTGTTT 3301 TAGTTTTAAT CAGTTTCTTT TTGGAAATCG GATATTTTTC TTTAACATGA GACAAATAAG TTTTATAAAT TCAAATCTTT TTGTATCATT AGTTTTTGTT 
3401 TGTTTTACTA TTTATTGATT TGAGTTGCAT ATACATTAGA AGATTAGTGA TTCTTTGAAA TGAATGTCAC AACTAAAATG CAAATGAATA TTTTTTAACG 3501 GGATTTGTTT TCTTTTCTTT TTTTTTCTAA AGATATGAGT ACATGACATT TTATAAAAAA AGGTAAAATC ACATAACAAA AATCAAGCCA CTCCCCGTTA 3601 AAAAAAAATT GTGACCAAGA CGCCATTTTT TCGGTATATT TTTTACAATC AATAAAAAAA TTGGATGGTA AAAAACTCTC CACACAGCAC AGTTTACGCC 3701 AATAAAAAAC CTGGTAAACA AAAAAAGCCC TGACGGTAGC AGGCACACGA ACTGCGCGTG CCCCTATTTA AAGCAACCCC TAAAAATTTG AAAATTGTAT 3801 GAATACCAAG ATAAAGAATA TAATTCCACA CCACGCTACC ATACCCATAA CTGAGTACCA TAAAAACTTC TCCCCTTCAT TTATTGTGTA TTGTTTTTTA 3901 TTTTATTCCA ACAATGTCTA TCAAAACGCG CCATGGTAAT AATGGTTCAC CAGGGTGTAC ATAAGACCCT CCTATATTTA AAGTACACAT CCGACTAGTA 4001 GTATATAATC ACAATGAGAA AAGATATAAA AAAAACCTAA ATGGAATACA GAGGGCGAGG GTATAGCACG CGGTGGTTTT TTTTTTTAAC ATTTTTTCGT 4101 TTTGTTTTAT TTTTATACGT TTTTTTTTTC GATAACTTTG GGTTTTGAAT TGTTTTGGTT CACTTTTATA CGAAGATTCT AAATCGGATT TGGTAAATTG 4201 AGAAATGAAT TCTCAATCAC CCTACGTAGA AACATTAATA CGTGAAACCA TTTTTGTTTT TTCATTTTGT TTTTGATTTT GCTTTTGTAA TATTTTATAG 4301 TTGTAAAAAC CCTTGGAGTA ATAACATTTC GCTCTCCACT GTGTTTCCCC TGGTTTTACG GCTTTGTGTA TATATACAGT ATTTATTTGG CTCGTGGCTT 4401 TAATATGGAA GAATGAATTA AAGATTTTTA TTTGAGGTAA TTCCTCTTGA CTGAAAGTGG ATTTATTTTA TAGCTAATGT ATTTTTGTCA TATGGATTTA 4501 TATTAATTAA AATCTTTATT TTTTTTCAGA TCGCTCTCCA TCGCAGTTAA ATTTGAAGTG AATCAAAAAA AATTTATCAA AACAGTTGCT ATTGAAACCA 4601 AAAACAAATA CAAAATGCAA AATTGGGATA CATTACAACC GACAGCCAGT TATGAACATA ACTGGTACGG TAATATGTTT CCAACAATTA AAACAGAATC 4701 GATGACTCAT TCGCCAACTA CATCACAATT AgAACAATAT TTAACAATGA AACAACAAGA AATGAATTCG TTAACACCAT CACCGCGTGC TGATATAAAC 4801 GGAACTTCAG ACATTCAAAA TTTCTTTGAT AGTCACAATC TGCAAAATGG CAGTTTGCAT CATAATCATC CATTAGGATT TAATCCATTA ACACCACCTG 4901 GTCTACCAAA TGCTGTTCTA CCAGCAGTTT CACATTTCCA TCATAACATT ACAGGAATCC ATGCAAATAA TCCAGCGAGT CCACTGGCAC AGCAATCCGA 5001 GGGAAATACT CAATCATTAA CACCACGCAA TACGCCACCT ATGGATATTA CTCCGCCAAA GTCACCCAAA TTGTACTCCG AATATGTTGA AAAGGATCAC 5101 GATATGATAT CAAATTCAAG TGACGACACA AAATTCCTTG AAAGCGATGA TGACTCATCG ATTCGTACAC CAATTTATAA TTCGCATGGC AAGATGAAAA 5201 ATTACAAATG TAAAAGTTGT GGATATATGG CTGTAACAAA AGTAGCATTT TGGGAGCATG CAAGTTCTCA TATGAAACCC GAAAAAATTC TTCAGTGTCC 5301 AAAGTGTCCA TTTGTTACGG AATTAAAACA TCATTTGGAG TACCACATTC GCAAACACAA AAATTTGAAG CCATTCCAAT GCGACAAATG CAATTATAGC 5401 TGTGTCAATA AATCAATGCT GAATTCACAT CGTAAATCCC ATTCTTCTGT GTATCAGTAT CGTTGTGCCG ATTGTGATTA TGCAACAAAA TATTGTCATT 5501 CTTTTAAATT ACATTTACGA AAATATGACC ATAAGCCCGG TATGGTCTTA GATGAAGATG GTTGCCCGAA CCCTTCGATA ATTATAGATG TTTATGGAAC 5601 ACGTCGTGGC CCCAAAATGA AGTCGCAATC CGGCGGTGGT GGTGGTAAAA TAAGTTCGGG AACAAAAAAA TTATCCGCAA TTAAAGCTGA ATTAAAAGTT 5701 CCATGTGGTG GCTCTCAACT ATCGGCGGCC TTACAAGGCC AGCTCCATTT TCCAGCATCT CCAGCTAAAA GTAGTAATTC ATCATCTTCG GAATACCCGG 5801 CGGTCTCTTC ATCCTCACTC TCACTAAGTC AGCAAGTTTA TAACCAACAA CAAAATCAAC AACAACAGCA ACAATACCAA CAACAGCAAC AGCAGCAGCA 5901 GCAACACCAA CAACAACAGC AGCAGAAACA ACAACAGTCA TTACCACAAA TATCCAATTT GTTACCACCA TTAGCCTCAA TTCTGCAACA AGGAAGAAAT 6001 ATGTCATTCT TTCCATACTG GAATATTAAT CTACAAATGC TTGCAGCTCA GCAGCAAGTT CTTGCTCAAA TGTCCCCAAG TATGCGTGAA ACTACCATTC 6101 AAAATCTACA GAATGGACAG TCTCAAGTTA TTGAAAATGA TAAAGATTCC GTCCAGGATT TCGAATGCGA GACAGACGAT GAATTTAATC GTCGTTCAAA 6201 TGGAAGCGCT ATAGACCTGT CACAATCAAA CGGAACGCCC ACCAAAATCA CAAATTTCAA TCAAAATACA TTTCAAATGT CAACCAATGT ATCAAATGTG 6301 TTAGCCGACA GTGGCATGCA GCAAATTAAA AGTAAAGAAG AAATCAATAC TCCAACAATA AGTTCATCCT GCAGTTCACG GCGCAAGGGA CGTGTTCTTA 6401 AACTCGATAT AAATGCAATC AATCGAAATT TAGTAAAAAG TCCCGAAATT CATTCCCATC AACCGCGATC GACAGAATCG CCATCGTCTT CATTTTTTGA 6501 AGAACCAAAA CTGCAAGAAA AATCGCCTCC TCATAACAGC ATGGATACCA ACAGCATTTC CATGCTAAAT TCTCCAGAGT CATTAGATCT TTCATCAACT 6601 GCCGTTTCAG CTCCTGTTCC ATCAGCATTT ACAACAACAA CCATTAGCAA CACTAATAAT AATTCAACAA CTCCAAACAC TACGAGCACA ACTAATACCA 6701 CAAGTAGTAG TAACATAAGT AATGTTAGTA GTAGTAACAG AATTAGCAAC AATCCGACCT CTTCTCAAGG CAACATTTAT GAGTGCAAAT ATTGCGATAT 6801 CTTTTTTAAG GATGCTGTTC TATACACAAT TCATATGGGC TATCATAGTT GTGATGATGT TTTCAAATGC AACATGTGTG GTGAAAAATG CGATGGACCT 6901 GTTGGACTTT TTGTTCATAT GGCCAGAAAT CCACATTCGT AGGCGTTTAA TTTTTTCGCG AATTGTAACT ATATATATGA CTCTATTCCT AGAAAAGGAA 7001 AATATTTATG GTAAATGGTT AAAATAATTT TAAGAGATTA ACTCATCGAA TTCTATTTAT TTAAAAAGAA ATAAAAAATG TTTATTTTCT GCCTTGTCAT 7101 CTTTTGTACA TTTTTCTAAA TACTGAAAAT TTAACTTAAC TGATTTGTAT TTTTTATCAG TTTAACAAAG TATTTTAGAC TTTTAGAAGT TGTAATGAAT 7201 TTTTTTAATG AAATACATTA ACTAAATGAG TTATAACAAA TAAGCACAAT GTTGTCATAA TTGTACATAA AAATGTTTTA TTTTAAGTTA ATACATTAAC 7301 ATTTAGTTTT AAAGTTTATT TTATTATTTT CGTTTTTTCT TAAAAGTTTT TTTTTTTTTA TTTAAATAAT TAATTTAGTT TTAATTAACC AAATTATGGC 7401 AATTAGTTTT ATCCTTATTT AAAAATAAAA CCATAAATAA AGGACCATAA ATGGTGGTAA AAAACCGTAT AAAATTAACC AAAGCCATTT TAGCCATTAT 7501 TATTATCCTA AACCATAGCC ATGGCCTTTA AAGGAACCAT AGTCAAGGAT ATATATATCC TTATATAACC AAAGGAAATA TTTTTTTTTT AAGGTAGGTA 7601 AAGTAAATCC CACAAGAAAA AAGGTGTTCC ATGGTAAAAG AATTGGACAT TTATTTGGTT AAATTTTAAT AATTTTAATA ATTTTAAAAA AAACCGAACT

7701 TATATTGGTG GTGAACTATT AAA

Source: Two different PCR products $(1 . .4557,2726 . .5470)$ amplified from independent genomic DNA templates, and phage Llu-hb ph2 (2726..7723). Alignment with cDNA sequences: Positions $1 . .50$ correspond to parts of the first exon of the Lonchoptera hunchback P1 transcript (SEQ13), and positions $4528 . .5280$ correspond to parts of the second exon of the Lonchoptera hunchback transcript. Two putative polyadenylation signals were identified in the genomic sequence $(7424 . .7429,7436 . .7441)$, and one NRE sequence (7249..7269).

\section{SEQ15 Empis hunchback, cDNA, partial P1 transcript.}

1 AgTCATGTCT GATGTCTTAT AATGAGAAGG ACGTGTGTGC ACTAAAAAAA AAAAAAAGGT GAAAACAAAA AACATTTAAT TTACACCGAA ATAAATATTA 101 TTTTTTAATT AAAATTAATA AAAAAATGAA TTAAAAGAGT TTAATTTTGA ATATCTTTTT TTTTTTTTTT GGATATTTAA TAATTTATAA GAATTTTTAG 201 TTTTAAAAAC GATTTATTGG AATAAAAATG TGTTAACGGA ATAAATTAAA AGTGTTTTTA TTAAATAAAA CAAAAAATAA AATTAAATTA AAATTAAAAA 301 AAAATTGATT GAACAATAAA AATAATATTA AAAATTAGCG CAAGAATTTT TCAAGAGATA ATTAAAAATT TGTGGATTAT AACGTTTTCA GTCGTTCAAG 401 TACGAAATTT AAAAGAGTTT TTCAATATTT TTGAGATCCA TAATGCAAAA TTGGGATTCA TCATTACAGC CAGCTAATTA TGAAAATAAT TGGTATGGTA 501 ATATATTTCC ACAAATTAAA ATTGAACCAC AAACAGCAAT TAATAGTAAT GATAATGGTC CACCATCAGC ATCATCATCA TCACTACCAC TATCAACACA 601 ACAACCACTA CCACCTCCAA CATCAACATC AATATCCGTG ATAGATCATT ATTTCAATAT TAAAAATCAA CATTTACAAA ATCAACAAAA AGAAATTGAT 701 ACGAGTTCAG TATTAATACA ATCACCACGC AATGATAGTA ATGATGAACA GCAATTTTTT GATAATAATA ATACTAAAAC TAATCATATA AATATTAATA 801 ATAATGGTAA TAATAATTTA TTGCATCAAC AATTGCATCA TCATCCATTA GGTTTCAATC CATTAACACC ACCTGGCTTA CCAAATGCAA TTTTACCACA 901 AATAAATTAT AATAATAATG ATTTGTCATT AGAATCAATA CAACAACGTC CATCATCATC GATAGGACAA TTAAATCATA ATCAACAAGA TATAAATATA 1001 ACAAAATTAG ATAATAATAA TTCATTAACA CCTCGAAATA CACCACCAAT AGATATAACA CCACCAAAAT CACCAAAAAA TGGACCAACA AATATGTATA 1101 ATAATAgtgG CAATAGTGAA TATTTAATAA TTGATAAAgA TCAAGAAATT TTATCAAATT CATCAGATTT AgTTGATAGT GATGATGATG AAATAATACG 1201 TATGCCAATA TATAATTCAC ATGGTAAAAT GAAAAATTAT AAATGTAAAA GTTGTGGTTA TATGGCAATA ACAAAAATTG CATTTTGGCA ACATACACGA 1301 TCACATATGA AACCAGAAAA AATTTTACAA TGTTCAAAAT GTCCATTTGT TACAGAACTA AAACATCATT TAGAATATCA TATTAGGAAA CATAAAAATT 1401 TAAAACCATT TCAATGTAAT AAATGTAATT ATACTTGTGT TAATAAATCA ATGTTAAACT CACATTTAAA ATCACAT

Source: 5' RACE product, amplified from an embryonic cDNA template. The sequence of an independent 5' RACE product, which was amplified from a cDNA template prepared from ovaries, aligns exactly with the embryonic cDNA but lacks the first 47 bp of the embryonic cDNA. 


\section{SEQ16 Empis hunchback, genomic.}

1 ATGAGAAGGA CGTGTGTGCA CTAAAAAAAA AAAAAGGGTG AAAACAAAAA ACATTTAATT TACACCGAAA TAAATATTAT TTTTTAATTA AAATTAATAA 101 AAAAATGAAT TAAAAGAGTT TAATTTTGAA TATCTTTTTT TTTTTTTTTG GATATTTAAT AATTTATAAG AATTTTTAGT TTTAAAAACG ATTTATTGGA 201 ATAAAAATGT GTTAACGGAA TAAATTAAAA GTGTTTTTAT TAAATAAAAC AAAAAAATAA AATTAAATTA AAATTAAAAA AAAATTGATT GAACAATAAA 301 AATAATATTA AAAATTAGCG CAAGAATTTT TCAAGAGATA ATTAAAAATT TGTGGATTAT AACGTTTTCA GTCGTTCAAG TACGAAATTT AAAAGAGTTT 401 TTCAATATTT TTGGTAAGTT TTTTTTAAAT TTTAATTATT GTTTTTAATA AAAAAAAAAT GTTTTTAATT TTAAGTGAAT AACAAGTCAC AAGATATAAT 501 TTCTACATAA AAACTAAAGC AACAAAAAAA AAGATGAATT TTCAAAATTA ATTATGAAAA TTCATCTTTT TTATTTATTT TTGCTAATTT TAATTTTAAT 601 TTAATTTAAT TTAATTACAA AAAATGTTAA GGAATATTTT ATATTTTGTC TTGCGCATTG ATTGAAAACA ATCTACGACA TTGTTGATAT TTTTTAATAT 701 TTATACTTAG TGTCACACAT AAAAAATAAC AAGTTTTGTC ATTAAGCTCA TCATTAAATT AACCTCTTCT AAAAAGGATA ACAATATAGA AAAAAAAAGT 801 CCAAAAAATA TATTCACTGA AAGTACTATG TTGTCAGATA GTGAAAGAAA TATGATGTAT ACATAATTTA ATTTATTCCT TTAACAAATT CATTTCTGTA 901 AAATTATCAT TTTTCAAAAA AAAATTAGCG ATTACCTCTC TATTGTAAAA TGTAATATTC GTTTCAAGTT TTATACAAGA CTATTTGCCG GTGTAGACTA 1001 TGACAATAAT ATATAAGTTT AAATAAATTA ATAGAACTTT CATTAACTTA TTTAAGTTGC AAAAAAAAAA ATTACTATTA AATTAGGTTT GCAATATCAT 1101 TATTTTGACA CTTCTTTTTC CAATTCTAAA GTAAATCCAA AGCACGTTAA TAGTACAAAA TGTAATTTAT CAATTGCGTG GGTAATCATC TGTTAATGAC 1201 ATGAAATGTA TTTTTTAGCT GAGTTTTATT TTAAGGATTT AATTTTATAT TATATTAATA TATGTATTAT ATTTTTTTTC TACTGGAAGT AGAGGAATTT 1301 ACTGGAAAAA GTAGATGTTC ATTACGTTTA GTTTATTATT CAAGAATTAG AGATTTAAAT TGTAATTCTT TAAATATCCA AGGATTAGGT ATTTATTACC 1401 TAAGCCTGGT ATATTTAAAG AATTACAATT TAAATTTTTT TCATTTTTTT GCATTGTTAT AGCTTATTAT GCCCAACAAA TTAGTTTCAA GCCAATTATA 1501 ATTAATGATT TTCTATGGTT TAGTAAGAAA ATGGTAATAA TTTAATATTA AGTTCTATTT TAATAAAAAT TACTAATTTA ATAACAATTT ACCACTTACG 1601 ACTTGTAGTT TTAGGTAATA TCACATATAT TTTCATATTA TTTATAATTA AAAAATGTAA ATTATATTAA AGTATATTAA AAAAAAATAC TGAATTATCA 1701 CTTTTTATTG AAAATTTAAA TTTTAGTAAA AATCTTATTA CAATAAAAAT GTAATTTCTA GTTTTAAAAA TGACTCAATA AAACTATTTA TAATTTTTTT 1801 TTATTAAAAA TGCATTTTCT TTTTTTGTTG TTGTTTTGCA CTCCCAGATT TTTCACGATC CGACAAAAAA AAATTGTTTT TTTTTTCTTC TTTTAACTTG 1901 GGTTCATTTA TTTTTGTTTT AACACGATAT TTTTTTTTTT AGAAAAAATT ATAATAATAT TTAAATCTTA CACAAAAAAA AATCTAAAAA TTCTAAAAGT 2001 TTGTTTTTAT AAAATTAGAA AAGAAAATCA TTTAAAAACA TCACTAAAAA CTCTATAATA AAAATTCGCG AAATTTAGCA ATGCGCATCT CCAACATCAA 2101 AAAAATGCAC ACACATTTAA AATTAATTTT AAAATAATAA TTCAAAGTCA GTAAAAAAGT TGGCAAAAAT TAAATGCATC TGAAAAAAAA ATTTATATTT 2201 TTTATAAAAA TATCTCACAA AAATGAAAAT GAAGAAAAAC TTGACCGGAA AATATTAAAA ATCACTAATA CATCCAAAGA TGATTTTATA TTTAAATTAA 2301 TTTTTTTTTT TTTGTTGCCA TAAATTTTAA AGCTAAATTC ATTTATAAAA TTAGTTTTTG GAAAAATTTA TTGTTTATTA GTTTTAAGTT ATTATTGCTT 2401 TGTTTTTGTC AGCCCTTGAC TTTTTCTTAC TTAACTACAG GTTGATTTAA TGGCTGTATA TGACGACTTT TAAATGAGTT TTAATATTCT AAAGATATCG 2501 TAAAAAAAAA CAATGAAATT TAAGCGTTTC AAGAAAAGAA AAGTCAATCT AACCTCAATG TTATTAAATT TGGGCTCCTA CCGTCTGGGA TCTACACCTT 2601 GAAAATCTGT CAAAGAAAAA CCGTACTTGA AACATTGTCT TAAAGTACAT ATCTTTAAGT AATTTTTTAC TGATTTAACT GACAAACAAA TAATTTGTTT 2701 GATATTTTCT TTGACTAATT TTCAAGTTGT ATACTGAAGT AGAGCCCAAA CGTGATACAT TAGATTTTTA TTGTATTGAA ATTAAAATCA TAGTTTATAT 2801 TTTCATTCAC GAAATAATTT AATCTACTGA AATATTTTTA ATTAAATACG TGCTAATTAT TTTCAGTAAT AGATTGAATT GTTGAGTAAA ATTTTCTGGT 2901 TATTTTCTGG TAATAGTTTA AATTTGTGCA AAATTTTCTC ATTTGGTTCA ACCGAATTTT TTGCCATAAT TGGTTAAAAT ATTTTAGTTT ATTGGATTTT 3001 TCAAATTAAT ATAATTTAGA TCTTCTAAGA ATATTAGCCC ACATTGTATT TAAATTATAT GCCACCAAAT TATTGTTACG ATATTTATTT TATTTCTTAA 3101 ATTTATAAAT CACCCTATGC TTATACCCTG TGTATGATCA TGGACCCCAC AAAAAATTAT ATTTAAGTTT TTGAAAAAGA TCTGAATTTA AATTATAAAA 3201 AAATAAATAA ATAAAATTTT AAGTTTTTTA AAGCTTTTTA AAGATTTTGC ATCTTGCGTA AACTTTCATC GAAAAAAATT TATTTTGCAT CCACCGGCAA 3301 TTTTTTTTTT TTTTTGCTTT TATGTATATT TTTTGCAGTT GGGATGCATT CGTTATATTT ACATCGCAAT GCCTGTATAC AAAAAAATAC ATTATAAATT 3401 TTACACTTGG CCTTTAGAAT TTTTACTTTT TGGATGTGTG TATTCTTTTT TAGTTTTGTA AATAATAAAT TTTAAATAAA ATTCATAAAA ATATTTTTCC 3501 TATCTTAAAA ATAGAATTTA CACAAAAAAA AAACGAATTT GAAAGAAAAT TGTGTTGGCG ATGCATTCAG TTTTGCGTTA TAATATGTTT TTAAATAAAC 3601 TTTAAAAAGC GTATTAGAGA GATTTTCTGA AAATTTATTT TTCTAAGTTT TTTTCAAAAT TTTATGGGAA CAATGGTCAC TTTTAGGATG GTTTCTGCGA 3701 AAATATTTAT TATGGTCACT TTTCATTTAA GGAAAAAACC CTTCAAATAT TTTTATAGAT GAAAAAGAAA ATGTTTTTTT TTTCCAAAAA AAAAAACAAA 3801 AAAATTGATT AACTTTATGT TAATTGCGGG TAATAAAAAA AAAAGAACCG GACTTGGAGT TAAATATAAT TAAAAGGTAT ACATACACCA GAAATCATTT 3901 TTTGAAAATC ATAATATAAT ATGTAAAAAT AATATTATTG TTAGTTTTTT CCCTTTTACT TTATTACCTG ATCTTTAAGG TAAGATGACA CTTATTTTGT 4001 CTGTATATCA GTTAAAAGGT CTTCACTTAA AATCATTTTT AATTTACATT TATATAGTAA AATTTTTACG ATATGGCTTT TTGTCTATGA GAAAGAAAAA 4101 TATAGAAATT TATTTGATTT TAATCTATTT AAGATGGTGG TTTTTACAGA GATATTTAAA AGTTTGCAAA TTAAAAAAAA ACTTGTCTTT TTTTTTATGA 4201 TATATCATAA AATTTTGTTT CTATCAGATT TAGTTTGGTT TTACTGAACA TTGTAAAGGA TTGTTTAAAT AGTGAACCTA AAACAAACTG AAATAAATAA 4301 AATTTAAATA TGGCAGGGTG ATTTATTATA TTTTGTGTAA GTGTAATAAA TTTAAAATGC AGAAATATAA CTTTTCATTA AATTTTTAAG TTCATGAAGG 4401 TCACTGCCAA ATTTGTATGT AAAGCACTCA CTCAATAAAA TTTTAAATTT CAAAACAACA ATACAAAATG ACAATTTCTT AATAGCCTTT AAATTATATC 4501 TGCTATCTCA TAAATAATGC TAAATTTATG TTAATTCAAA ATGGGTCAAT TATATACAAA TTCATCTTCT GGTACAGAAT TTTGGGTATA ACTAAATTAA 4601 CTTAAATTGT AATATTTAAA TCTAAATCGA TTAATTATAC AATAACGACA AATATACGTT TGAGTTTAAA ACTACTTAGA TTTCAGCACT TTTTCAGATT 4701 CCTGCAGCTT TGCTTTCGAG CATATGGAAG TTCTCATGGC ACCGAGAAAT TATCAAAACA AACTCAAAAA AATATTTGAA AAGAAACTAA AGGTGCATAG 4801 CAAAAAACTA ACGATTTATT AATTTTTTTA AATTATTTTC CTTTAAATAG TTCCACAAAA CTATATTTCA ATTTTCTCAT ATTTATCCAC CATTTTCTAA 4901 TTTTCAGTTT CGACGACATT CTTTGACACA ACCCTCTTTT TATGTGAAAA TTCCATAAAA AATATATTAA TTTCATTTTA AAAATTAATA TTTATTTTCG 5001 GAAATAATTT TATTGTATTT CAAATCGTGG AAATAAATTT TGAAATAAGT GAAAAAAAAA CAGTGTGAAT TGGTGGATGC TTCTTACTTT TTATCCTCCC 5101 ACCAAAAAAT GTTTTATGAC AAAACTACAA CCGCGAACCA ACAAAAAAAA TTATAATTTT TTGTGAGGTT TAAGAATTCC AAAAAATATA TACGAGGGGT 5201 GAGTTAAACA CATTACAAAA TTGTTGCCAG AGGGGTCGTG TAGTCAATAT GAGCTTATAT ACTTTAATGT TGAGTATAAA AAACAAAGAG GATAAACTCA 5301 TATATATGTC TTTATATAAA TTTAAGTAGA TCCTAGGGTC GATTTATAAA TATTACATTA TTATGATTAT TTTTTAGTTT TTTCAGTATT CAGGAAGGG 5401 GAGAATTACA TTATTATTAT TATTACATTT TTTTTTCAAC ATTGTGATTT TAATGTTGTT GATGTTACTT GAATTAGGTA TGAGATGGTT TTTTTATGTG 5501 TATAATATAA ATTTATATAT ATACATATAG TAAAAATATA AAATTTGGTT GTGAGACACG CGCAGTTCTT ATGCCAACAT TACAGAATTT ACCCCCTCCT 5601 ATATATTATG TATACAAAAT ATATTTTTTA TTCATGTCTA CCCCGAAGGA TATGAGTTTT TTTTTTGATT TTTATAAAAA AATACTATTT AGATTAATTT 5701 TTTATTCAAT TCTGGTTGGT TGGAAATTAT CAACGTTGTT TTTACGATTT TTTTTTTAAA TGGCGACAAG CATAATGGCG TATATAATAA TTGTATTTAA 5801 AAGAAAAATA AAAAGTATTC TAATATTAAT AAATTAAATT TTTTAACTTT CAGAGATCCA TAATGCAAAA TTGGGATTCA TCATTACAGC CAGCTAATTA 5901 TGAAAATAAT TGGTATGGTA ATATATTTCC ACAAATAAAA AT

Source: PCR product, amplified from genomic DNA. Alignment with cDNA sequences: Positions $1 . .413$ correspond to parts of the first exon of the Empis hunchback P1 transcript (SEQ15), and positions 5854..5942 correspond to parts of the second exon of the Empis hunchback transcript.

\section{SEQ17 Haematopota hunchback, cDNA, P1 transcript.}

1 GTGGAATTAA GACAAAAATT TTTTCGTAAA TGAGTTTTTT TTTTTGGGAA AATTAATATT TTTTTCGCAA ATGAATAAAA AAAAAAAAGT AAATAAATGA 101 CAGTTTTGAA AAAAGAAATT GTGAATTTTT GTGTTAATAA ATTTATGTTT TCGAAAAAAC TTATGTGAAG ATAAAAACAT TTAAAGTGAT TTTGTGAAAA 201 TTATGAAATA ATTTGGACGC AATTTACGTG TTTGTTTAAA AAAAAAGGAT ACCAAATTTG TTGAACAATA TTCACCGGAA TAGGATAGAA AGAGACGTAT 301 CTCATCATGC ATGGTTGGGA ATCATTGCCG CAAGCAACAT ATGATCATAA CTGGTGTGGA AATATGCTAC CAATTAAAAC AGAACCACAA ACTACAACAT 401 ACCCATCAAT GGAACATCAT CATATGCATC ATATGAATCA AAAAACAAGT TCATTGGGTG GCGGAAGTTC ACCACTTTCT ACCCCCAGCA TGGATGGAAT 501 GGAAACACAA AACTTTTTCT ATGATCAATT TAGCAGTTTA CATCGACCAC TTGGTTTTAA TCCACTCACA CCACCTGGAT ATCCAAATGC TATGATACCA 601 CAATCGAGTT TGCATCAAGA TTCTATGTTA AACGTAGACC AATCACCGTT ACAGCAACTT AATCATGGTA GTATATCACA ATTTGCTGCT TTTGCCAAAA 701 ACGATGGTAG TAATCCATCG TTAACACCAA GCCATACCCC GCCAATGGAT GTTACACCAC CAAAATCACC AAAATTTCCC GTTGATATAC CAACACCGGA 801 AAAgGATAAT GATTTAAATT CAAATTACAA TGATTCAGAA GATACACGAT CATTGGAAAg TGATAATGAT GATGAATCTA TACGTACACC AAAGATAAAT 901 TCACATGGA AAGTTAAAAA ATTCAAATGT AAACAATGTA ATTTCATAGC TGTAACTAAA CTAAGCTTTT GGGAACATAC TAAAGGTCAT ATAAAACCGG 
1001 AgAAAATGT AAAATGTCCA AAATGTCCAT TTGTAACTGA GTACAAACAT CATTTGGAAT ATCATTTACG AAATCATGAT GGCTCGAAAC CATTCCAATG 1101 TAACAAATGT AGCTATAGTT GTGTTAATAA GTCAATGCTA AATTCACATT TGAAATCACA TTCGAATATA TATCAGTATA GATGTGCTGA TTGTAGTTAT 1201 GCTACTAAAT ATTGTCATTC ACTAAAGCTG CATCTACGAA AATACGGTCA TAAACCAGCC ATGGTCCTGA ATGAAGATGG AACACCGAAT CCACTGCCAA 1301 TCATCGATGT CTACGGTACA CGAAGAGGCC CGAAGATGAA GTCATCGAAG AAACGTGATC CACCATCACA GCAACTCTTC AAACAGGAAA CACAGAATGG 1401 ACCATCATCC CTCGCACAAA ACTCTAAAAC TCCACCCCTT CAGCAACAGC AACAATCGCA GCAATTTCAA GCGACTGTGC CAACCCCATC ACCGGCTAAT 1501 CTAATGTCCA ATTTCCTGCC TACAACATTA GCGAGTATGT TGCAACAGAG CGGCAACACG ATGCCATTCT TCCCGTACCT GAACCTAAAC CTTCACATGC 1601 TAGCGGCACA ACAGCAAGCG GCTCTCGCCC AAATGTCACC AAACATGCGA GATGAGACAA CGAACATGAG TAAATGTGAG AGCGATGAAG AAGATGCTAT 1701 GAGTGACTAC GAAACTGATG AACGATGTGA GAGTCGAATC GATAACGATG CCATGGATCT GTCGCAAACA ACCCCAACGA AAAATGTTGC CAACCAGAGC 1801 GACCCCATTG AGCCACCCAA AGAAATACCA ACAACACCCT CAACAGTTAC ATCAACATGG CGGAATCATA GGAGGAAAGG TCGCGCGTTT AAATTAGACT 1901 CGTCAGTAAC ACCCGCAGAA AATGAGAATC TAACAATGGA CACCACCCTT CTCAAGAAGC AACCAACGGA GGTTATTGAA ATGGATAACT CAAGTCGGTT 2001 GGAAATGTCG GGGGATGAAG ATGTTCCAAC ATCATCATCA TCGGTGGTGT TGGAGAACAA AGACGATGCT AGTGATGAAA CAAATAAGAA ACCAGAGAGC 2101 AGCACAACAC CTTCCCTGGA AGTAGAGAAT AAAGAAACAT CAAAGAGTAC CTCACCGAAC AATGCTAGTA ACTGCACGCA AGAGAACTAC GAATGCAAAT 2201 TTTGCGGCAT CTCTTTCAAG GATGCTGTCC TGTACACTAT CCACATGGGC TACCATGGAT ACAACGACGT CTTCAAATGC AACATGTGCG GCGAAAAGTG 2301 TGAAGATCGT ATATCATTCT TCCTACACAT TGCCAGGAAC CCACACTCAT AAAGTTCTGC ATCTATAAAG TCGAATTTTA CACTACGAGC GAGAAATTTT 2401 ACGACTTGAG CTTTCCAACG AATGATTTTC ATTCGTCAAT TTTGTCCGTT A

Source: 5' RACE product (1..1091), 3' RACE product (1065..1433), and an additional PCR product (221..2451), all amplified from a cDNA template prepared from ovaries.

\section{SEQ18 Haematopota hunchback, genomic.}

1 agCAAATATA ATATTACACA TGAGCGCCCC AGAGTACAGC CATTTGTGGG ATCCCTGCGg AACAAGAGCA AAATTTCCTT CATATTCTTA TGCTTCGGTA 101 GATTGACGAG GAGAAGACTT GGGTCTGTGG TCTTAAAACC AAGTGAATTG TAACATATGT ACATGTCCCG TTGGATACCT GCTAAAGTTC TCCGAGAAAA 201 TAGGCTTGCT GAACGAGGAA TAAAGTGGAG AAGGGAAAAA CAGACCACTG GATGGAAGTG GCAAGGGGCC AAAACAGAAC TGCCCCACCT TTCTAATGAA 301 ACAAAATGGG GTGGAAGCAT TCATTTTACT AAACTTTTAA CTAATTTGAT AAAACTTTTT TGGCAAATTT TTTTTATTTA TTGTTTTTTG TTATTATATT 401 TAATAAATAC GCCAAATAAC ACATTTTCCG TTGGTCAAAA GTGGAAAACC TTAAAAACAC AAATCAGCTG AGAGAAATTT TTCAAAATGT CATCAAATGA 501 GGGGAAAACA AACATTTTCA GCTATAAAAA GTCCTTGGCT GAATTCCCAT TTGTCAGCGC TAATGCTGCG TTTACACTCG AAACTCACAC ACACCGCACA 601 TGCAAACGTG AATTTTCCCC TCCAGGACAC TAATTTCCTC TCACATCAAA TACTCGTCTC TGATTGGTTC AAAATCGGGA AAACATTCTA AACGATGAAG 701 AGAGACAGCA ATACGAAACA AAAAATGCAC AGAAAAAAAA TTTCTCAAAT ACCATCGCAA TCCGGCATTT TTCCGTAATA ATTTTTCTTC AAAAAGTGAA 801 AAAAAAACGA AGATGGGAAA AACTTTTTGA AAATGCGTTT TCGGAAAAAC GAAGGGGCAG GGGTTGGTTA ACGAACGGAA CGTGTCAGGT CGATAAAAAT 901 CTTAAAATTT TTCAGTACGA ATCGGGATGT CGTGGAATTA AGACAAAAAT TTTTTCGTAA ATGAGTTTTT TTTTTGGGAA AATTTATATT TTTTTCGCAA 1001 ATGAATAAAA AAAAAAGTAA ATAAATGACA GTTTTGGAAA AAGAAATTGT GAATTTTTGT GTTAATAAAT TTATGTTTTC GAAAAAACTT ATGTGTGAAG 1101 ATAAAAACAT TTAAAGTGAT TTTGTGAAAA TTATGAAATA ATTTGGACGC AATTTACGTG TTTGTTTCAA AAAAAAAGGA TACCAAATTT GTTGAACAAT 1201 ATTCACCGGA ATAGGATAGA AAGGTAAAAC CAAAATTAAT TAAAAAAATA AATTCAAATT CAAAAATTTC TAAAAATTTA ATGACACATA ACGGGACTTT 1301 GGCTAGCGAG CCGTGAGGCT TAATTTAGTG GAATTTTCAA AAAAAAAACA ATATTTTATT TTTATCAATA ATTCATTAAA AAACTTGAAT TGTTTTAATG 1401 CATCTGACCC CAAAAAAAAA CACAAAAAAT CCCTGCAGGC TATTGAACGA ATATTCCCCA AAAATTCCAA AAACGAACTT GACAGTCGTC GAGGTTGTTT 1501 CCATTCCGTT GAGGTTAGAA AAAAAAACAT TTTTTTTTAT TACTCGATTT TGGATTTTCA ATTTCCAATG ACCTTCCGGA ATGCAGGCAA AATTTGGTCA 1601 AAAATTCACA AAAATTTTGT TTACTTTAAG AAGCAGTAAA AACTAATGAA CGGATTAGAA AAAATTTCGG GGAGGGCAAA AAGTCAATTA CATCCATAAA 1701 CTGCACTGCA TCCCTTGCAT TGTAACGGCT TTAAAATGGG GGTGGATACG TGTATAGGGT CTCATAAAAT TTTATTGAAA TATTTGCTTA AAAATACCCT 1801 TGCGAACAAA CATTTTTATA ACGACGGAGA GAGACACCAT TTTGTTTGAC AATTTATTTT TAAAAATCAA TTTTTGGGGT TGGTTCATAG CAGGAGGGGT 1901 AAATATAACG AATCAAAAAC TAAAAAAACG AAAGACGTCC CACTTCTTTA CACTCCTCAT TGGTGTTGAA ATAAGTACAT AAAAAAAAAC ATTTGTAGGC 2001 ATATAAAAAA TACTAAGAGG GGTTGCTCAA CACAAAAAAA AAATTTCATC CCCTAAATAA AATTTGCTAA AAAAGTAAGA ATCGCGTACG TTTGCCACGA 2101 GAACTGCGCG TGCCCCTTCA ATATAAACAT CATCCGGGG TTGTGTTGTA TGGTAGGGTG GTGTTGAATA CATTTTTGTT GTTGTTAAAT CACTCAAAAA 2201 GGAAAAAAAA AAGAAGCAGG GTAACTACCC TCGTGTTGTC ATACACATTT CTATCTCATT TTATCCAATA CACATTCGTG AGTAAAGTCA CCATTTTTCA 2301 AGTATATAAG CGTATTTTTA TACTTAGAAT TCTTTAGTTT TCTCCCTTCT GTACCAAAAA CCATCCCTTC AAGCTTAATG ATCCTAGTAT GGGGTGGTAA 2401 AATATAGTAA AAAAAATGTC GAGGGGAGAA TTTTTTTTCT TCTGTTCACA ATTCTGGTAT TTTTTTCCAT TTTGGAAATC ACATTTTTTT TTATTCTGTT 2501 CCCTGTGATG CTGCCCTTCC TTTGATTCAC TTAAACCGAT TGGAAATCAA GTAGAGTCCT TGAGAGGGAT GAGGTTGTGA GGGAGACTCT AGGAGGATAA 2601 AGAAAATATC AACCCCCGCG TCTGGTGGAA CTTATTCATC CATAGGCACT GAAAGGCGAC AAAAACGACA TTAAGGTACC CCAAAAAGGG AAGCTTTTAG 2701 AAACTTGTAG ACCAGATAAC GCACAATGAT GTAGAAAAGA TGTAAATGAA TACTAAAATC GTTAGTCTGA ACTTCCTTAT CATTAGTTAT TAGAATCTAA 2801 TCATGTATAG AATTCATTGA CAATAATCAA ACTTTTATTT ATGCTTCATT TCAGAGACGT ATCTCATCAT GCATGGTTGG GAATCATTGC CGCAAGCAAC 2901 ATATGATCAT AACTGGTGTG GAAATATGCT ACCAATTAAA ACAGAACCAC AAACTACAAC ATACCCATCA ATGGAACATC ATCATATGCA TCATATGAAT 3001 CAAAAAACAA GTTCATTGGG TGGCGGAAGT TCACCACTTT CTACCCCCAG CATGGATGGA ATGGAAACAC AAAACTTTTT CTATGATCAA TTTAGCAGTT 3101 TACATCGACC ACTTGGTTTC AATCCACTCA CACCACCTGG ATATCCAAAT GCTATGATAC CACAATCGAG TTTGCATCAA GATTCTATGT TAAACGTAGA 3201 CCAATCACCG TTACAGCAAC TTAATCATGG TAGTATATCA CAATTTGCTG CTTTTGCCAA AAACGATGGT AGTAATCCAT CGTTAACACC AAGCCATACC 3301 CCGCCAATG ATGTTACACC ACCAAAATCA CCAAAATTTC CCGTTGATAT ACCAACACCG GAAAAGGATA ATGATTTAAA TTCAAATTAC AATGATTCAG 3401 AAGATACACG ATCATTGGAA AGTGATAATG ATGATGAATC TATACGTACA CCAAAGATAA ATTCACATGG AAAAGTTAAA AAATTCAAAT GTAAACAATG 3501 TAATTTCATA GCTGTAACTA AACTAAGCTT TTGGGAACAT ACTAAAGGTC ATATAAAACC GGAGAAAATG TTAAAATGTC CAAAATGTCC ATTTGTAACT 3601 GAGTACAAAC ATCATTTGGA ATATCATTTA CGAAATCATG ATGGCTCGAA ACCATTCCAA TGTAACAAAT GTAGCTATAG TTGTGTTAAT AAGTCAATGC 3701 TAAATTCACA TTTGAAATCA CATTCGAATA TATATCAGTA TAGATGTGCT GATTGTAGTT ATGCTACTAA ATATTGTCAT TCACTAAAGC TGCATCTACG 3801 AAAATACGGT CATAAACCAG CCATGGTCCT GAATGAAGAT GGAACACCGA ATCCACTGCC AATCATCGAT GTCTACGGTA CACGAAGAGG CCCGAAGATG 3901 AAGTCATCGA AGAAACGTGA TCCACCATCA CAACAACTCT TCAAACAGGA AACACAGAAT GGACCATCAT CCCTCGCACA AAACTCTAAA ACTCCACCCC 4001 TTCAGCAACA GCAACAATCG CAGCAATTTC AAGCGACTGT GCCAACCCCA TCACCGGCTA ATCTAATGTC CAATTTCCTG CCTACAACAT TAGCGAGTAT 4101 GCTGCAACAG AGTGGCAACA CGATGCCATT CTTCCCGTAC CTGAACCTAA ACCTTCACAT GCTAGCGGCA CAACAGCAAG CGGCTCTCGC CCAAATGTCA 4201 CCAAACATGC GAGATGAGAC AACGAACATG AGTAAATGTG AGAGCGATGA AGAAGATGCT ATGAGTGACT ATGAAACTGA TGAACGATGT GAGAGTCGAA 4301 TCGATAACGA TGCCATGGAT CTGTCGCAAA CAACCCCAAC GAAAAATGTT GCCAACCAGA TCGACCCCAT TGAGCCACCC AAAGAAATAC CAACAACACC 4401 CTCAACAGTT ACATCAACAT GGCGGAATCA TAGGAGGAAA GGTCGCGCGT TTAAATTAGA CTCGTCAGTA ACACCCGCAG AAAATGAGAA TCTAACAATG 4501 GACACCACCC TTCTCAAGAA GCAACCAACG GAGGTTATTG AAATGGATAA CTCAAGTCGg TTGGAAATGT CGGGGGATGA AGATGTTCCA ACATCATCAT 4601 CATCGGTGGT GTTGGAgAAC AAAGACGATG CTAGTGATGA AACAAATAAg AAACCAGAGA GCAGCACAAC ACCTTCCCTG GAAGTAGAGA ATAAAgAAAC 4701 ATCAAAGAGT ACCTCACCGA ACAATGCTAG TAACTGCACG CAAGAGAACT ACGAATGCAA ATTTTGCGGC ATCTCTTTCA AGGATGCTGT CCTGTACACT 4801 ATCCACATGG GCTACCATGG ATACAACGAC GTCTTCAAAT GCAACATGTG CGGCGAAAAG TGTGAAGATC GTATATCATT CTTCCTACAC ATTGCCAGGA 4901 ACCCACACTC ATAAAGTCGA ATTTTACACT ACGAGCGAGA AATTTTACGA CTTGAGCTTT CCAACGAATG ATTTTCATTC GTCAATTTTG TCCGTTAAGT 5001 ACAAACACAA ACGCAAGCGC TTTGTTGTGT AGAATTCAAC TTGAAATTTG TTTCAAATAT GTAAATGTTT TGATGTTTAA GAATATTTAT TTGTAATTTA 5101 CGTATATAAA TTTAAATTCT TGTAATTTAT TCAAAAATTG AGGCCTAATA AGAGCGAAAT TTGTTTGGTT TTGAAAATGG AGT

Source: Genomic DNA, phage Hpl-hb phB. Alignment with cDNA sequences: Positions 932..1223 correspond to the first exon of the Haematopota hunchback P1 transcript (SEQ17), and positions 2855..4998 correspond to the second exon of the Haematopota hunchback transcript. In the genomic sequence, one putative NRE sequence (5022..5043) was identified. 


\section{SEQ19 Clogmia hunchback, cDNA, P1 transcript.}

1 CGGACCTTAC GTTCTGTATT CAGTCTCTGA AAACTAAGAA CGTATTTTAA GTGTTGTTTA TTAGTTAGTT AAAAATCTGC TCTCCCCAAA AACGATTGTG 101 CTTATCTCCG TTCTGTGAAT ATTATCTATA TTAGAAGTGA TTATTATATT CGCAAAACTA ACGACTATGT GAGATGTAAT TGTGATAAAG GATACTCTAT 201 TTGTTGTGCC CCATCTATCC TGGATTACTC GTATATTTCG ATTATTGTGC TAAAAAACTT ATTGTGATAG TGAAATCCCG GCAGTGTCCA GTTCTTTTTT 301 GCACACTTTT CCAACTACAA AAAGTCAAAA TGCATAGCTG GGACGTGATT CCTCAGACCA ACTACGAGAA CAATTGGTAT AACAACAATT ACCAGATGAA 401 AACAGAGCCC CACGATGGGT TCAACGGGCA ACAGCCCAAT TCCCCGCAGA GCATGGACAG CATTCACCCT GAAACACATC ACAGTTCTCC AGTTCAACAA 501 CAACATATGA TGTTCGATTC GTCCAATATT ATAAACACCA TGACCCAACT ACACAACGTT CAAATGCAGA GACAGACCCA CTTCAATCCC CTTACTCCTC 601 CGGGTTATCC AGGCGCTATG ATCCATCCCC AAAACTCTCA GGCAAACTCA ACACCATTTA GAAGCTTTAC AAAGGGACTG GACTCGATTC CTTTTGGAAA 701 TAATGTATCC AACTTAACAC CGAGTCACAC TCCTCCAATG GACATAACTC CGCCAAAGTC ACCAAAGTTC AACGGCAAGG AAACCCCTGA AAAGGATTCT 801 CTAAAGCAGG ACCAAAGTCA ACTTCTAAAA ACCCCAATCC AGACGAATGG AAATGGAAAC CAGCAATCGA CGTTCGACTC TGGCGAAGAC AGCCACTCAA 901 TTCCCGATAG CGATCTCCTT GAACCGGTAA TCACCGACGG TGCGGACGTA GATGACGAAA ACGATGCTGA AGAGGACGAT GACATTCGCA CTCCGAAAAT 1001 CAATTCGCAC GGTAAAATGA AGACGTACAA GTGCAAACAG TGTGACTTTA TCGCAGTTAC AAAACTGTCC TTCTGGGAGC ACAATAGGAT CCACATCAAA 1101 CCTGAGAAGA TGCTCAAGTG CCAAAAGTGT CCCTTTATCA CCGAATACAA GCACCATCTT GAGTACCACC TGCGAAATCA CAACGGATCA AAGCCCTTCC 1201 AATGTAAACA GTGTAACTAC TCCTGCGTGA ACAAATCCAT GCTCAACTCA CACATGAAGT CGCACAGTAA TATCTACCAG TACCGGTGCA AAGACTGCAA 1301 CTATGCAACC AAGTATTGCC ACTCCTTGAA ACTGCATCTC CGCAAATATT CGCACAATCC AGCCATGGTG TTGAACTCTG ATGGAACGCC AAACCCACTG 1401 CCGATTATTG ATGTCTATG AACGAGAAGA GGACCAAAAg TAAAGTCTCA TAAGGATGAA GGAGGGCATA ATTTACTTAA CTCAAACATA AATACCAGTA 1501 GAAGGAGCAA GTCAGGGAAA CGGGACAGTT TTCCGAATTT CGAACAGTCT CAACATGTTT CCAATAATCC ATCAAGTCAG GCTTTGGCAA TGTTGCCCAA 1601 TTTGGCCAAC ATCTTCCAGC AGAGTCCCAG TATGCCCCTG TTCCCCTACC TGAACCTCAA CTTTCACCAC ATTCTGGCCC AGCAAAAGGC AGCCCTTTCA 1701 CAAATCTCCC CATCAATAAA TGGGTGGCAA AATGAGGAAA ACTGCAACGA GGAAGAGACT CCAGAAAAGG AGGAAGACCC CAAACGAATG TCTGCCCTTG 1801 ATCTCAGCAG CAATCCTAGC ACCCCATCAA CAATGAGCCA AGTTAAACAT AAGCGCAAAg GCAGGGCATT CAAATTAGAG CTGATGAAGG AGAGTTCCGA 1901 TGACGAAGAA GGCCAAACAA TTCGGACTTT AgGGGAGATC AGGAGCGAGC TGGAGACACC AAAACCAGTT CAACTTCAGT TACCCACCTC GAGCACCACC 2001 ACACCTCTAA AGACTACCTC TGAAGATGAT TCCACATCGG TGGAACCTTT GCAGAATTTG TACGAGTGCA AATTTTGTGA TATCTCATTC AAGCACGCCG 2101 TTCTCTACAC AATCCACATG GGCTATCACG GTTACAACGA CGTCTTCAAG TGTAATGCGT GTGGCAAAAA GTGCGAGGAT CGAGTTGCGT TCTTTTTGCA 2201 CATTGCTCGG GACGCACATG CGTAAGTCAT GTCACAAAAT CACAAAACCT TAAAATAAAT CCATAAAACG TTTGTACAGT CCAGGAAAGT GTGATATTAC 2301 GACAATAATT CCTAATTTTA AGTATTTTAT ACGTATTTTT ATGTAATTAT CTTAAGTATT TAAAAAATAA CAAAATCGAG AGAGGAAGAG GCTAGTTCCA 2401 AAAGTTAAGT TATGTACACC AAAAAAGAGG AGGGTAAAAT CCGATCCGAA AACCGTATAG TCATTATGGG GTGAATTTTT TAGTTTTAAC ATATTTTTTA 2501 TTTGTGTTTT TCATTCCCTT CGAATTAATA CGGACAACTC CTCTTCCCAA GCCAGGGCTA GTGAGGGTTG TTGTCCTATT TGGTCGTTGT ATAGATTGTT 2601 GTTATATTAC CAAAATGTCC CTAGGAGTCT TTTTTGGCTT ACAAGTGTAT AAGAGACACT CCTTTCTGAg CGAACCAAAT CAAAATGTCA CCAAGGATAT 2701 CAAATTAGT TTGTAAATTT ATTTTAGAgA TAAATTGTAT ATATTAATAT TTCAATCATG TAGTTTATAT ATTTATATAA TGGTTGATAA TTAAACAATA $2801 \mathrm{AA}$

Source: Lambda ZAP clone, isolated from a maternal cDNA library (Schmidt-Ott, unpublished). One putative polyadenylation signal was identified in the in the putative 3' untranslated region (2797..2802), and one putative NRE sequence (2584..2604).

\section{SEQ20 Clogmia hunchback, genomic.}

1 TCTTCAAATT TACACACAGT ATATCTCTCT ATATTATCTG CCTCTGTGTC CTAGTGGTTA GTACCCTGGA CATCCTCCTC GAGGTCGTGG GATCAAATCC 101 CGGGCGGATA TTTATCTAGC GCGAAAAAGT TTCCATTTGT GTGTATATCA ACAACATTGC GAAATAATAT TCATAGCGAA TTATAAATTT GTGATGGCGC 201 AAACAAATAA ATAAAATGGG CGGATTGACT GGATCCGTTT ACAACCGGTC TAGGTGCTAT TAACTGACTA CTAATACACT GAAAAAACAA ACAGTCGTGT 301 ACAGTCTAAC ACCCGTTGGT TGAGGTTGAG ACTACCCCTA GTTCCGTCGT GAATGTCGTG GGAATGATTA TTATTATTAT TATATCTCTT TACAGATTAC 401 AACTTTAGGT CAAACCAGAT TTGCAAATAC TTGAATCAAT TTTGCGAAAT TCAGAAAAAA ACGAAAAACC GCAAATGCCG AATGGGTCAG CGTTATAGCC 501 ACACCCCCCT TTCCTCAGCT ATGGGGCATT ATTTCAACAA TCTATTGATG GGGGTCGTTA GACAATCCGA AATATATTCA GAACTGCGAA AATTTGGGTC 601 GCGAAGTCAT AGCAGGCCGG CAGTAGCTCA CATTGAAAGT CTAAAAACCC TGATTTCGAA AAATGAGGAA ATGGTATTAA TAATTTTCGA TTTTAAAAAA 701 AGTAATAAAG ACAATCGAAA ATGGGGGTTA TTATGCATTA TTAGTGATTA AATGGGCACA ATAGGACAGC TAATAAAGTC ACAGTTACGA GTAACATTTA 801 CTGACAAAGC GGGTTTTTAG GCTACTACTA GACGTAGCTA AAAAACGGCT CGATGGATTT AACCCAAACC ATATTAAAAT AAAGATTATT AAATTCCCTA 901 CAAACCGTCC ATACATAGTT TTAGAGTTGG ACTGCTGGAT TCTAGTCATT TTGGCACTAA AGAAAATGTT TAATTTGCTC AATTTGGATG CATTTTAATT 1001 CCATATAGTA ATTCGGCCAA TATTTTTGA TTATTTCATA TTCAAAATGA TTCGAAATTG ATTTAAGTAT TTGCAAATCT GCTTTGACCT AAAGTTGTAA 1101 TCTGATTAGA GATATACTGT GTGTAAATTT GAAGATCCAG CTTCTTTTGG CTTCAGAGAT AACAAGGATA AACGGGTGCG GCTATAACCT CAATTCACAC 1201 TGTATCTGCA CAAAGGCAAT AAACTTGGCA AAAAATGGGT TTTTTCTTGA TCATCTTTAT TTTCATCGCC GCCGATGTCG TTGTTTTCCC CATTCCTAAT 1301 TGTCTTCTTT TAATAGTGTC CTCCCCCGTT TTGTGTGTCC TTTCCACGAG TTTCAGTCAC TGGCAGAAGC CGTGGCATTG GTGGACAATG GGTCGAATTT 1401 ATCTATTGG CATAATAATC ATTTATTTGC TCAAAATTGC CATTAGACAC CTCTCACACT TTGGCTCCTC TTCATTTGTT GTCTCTTTCC AATAAATTGC 1501 AATTTTTCAT AGACCAAAAC TCTCCCTCGG TCCATGTCAA TGGAACAGCA GTGAAGGGAT TAGCACAGCT GAGGATAAAA TGCGAAAAAA CCGACTAGGG 1601 GAAATTGGAT CTGGCTTGGT CCACAGATTT CTTCTCCTCT CCTTGCTGAT GATGTCGAGG GGTGGGTGGT GTGCATTTTG GTGAAAGTAG GTGGCAGAGG 1701 GTTGTGTTTT CTGTCAAAAA GTATAAGAAT TGAGGGGAGC CGCAAAAAAC CGTCTACGTG CAAAAAGAAT TCCCATGGCA ACCAGAAAAA TAAATGGGCA 1801 TTTCTCTTAT CGGAGACAAC GGAACAATCA TGGATTATTA TGGAATGGCA ATATTATTTC GCTTTGCAGA AATAGAAAAG TCTGCTTTTG ATGATGTGAA 1901 TTAAAACAAA TGGCAAAATT ATAATTCTTC AATTTATATT AAATGCATTT TATTCCAGAA GATCAATCTC TAAAATATTA ACTAAAATTT ATAATCAAAA 2001 AATAACTTTG AAATGCTATC TTTACATCGT ATCATAATAA TTTAAAAGAT AAAAATACTT TCATTTTTTA TATCAAACCC AATTATCATC CCTTTAAATT 2101 CCCCAGTTCA TTCGCATACA TTCCCATCAT CATCCCCTCT TCAATTTCCA CCAATAGTAT TCCAGCAAAC ACATTCAACA AAAAAAATTC AATACAATCA 2201 CATCCCCTAA ATGTTACACT CGGCGACAGT GGAATAGGAC CTATTTAGGC GCATGCTTTA GATTTGTTTG AATGTAGTGT TAATAATTTT TTCTACAAAT 2301 GTATTTTAAA AGATAAATTC TTATATGAAT ATTAACTAAT TGAATTTGCC TTAAACAAAA ATTTAGATAT TTCAAAACAG CCGTTTAGTT TTTTTGAATA 2401 TATTTTAATA TATTTCAAAT ATTTTTGTTA CATAAAATTG ATATTACGGT TTTAACATTA CATTTTAAAA ATGATTAGTT TTTTTCTTTA ATTTTAAAGA 2501 AACGATTTTC CTCAAATTAA TTAAATTCGG TATATGTTCT TGACATTCTG GAAATTTACT TAAATAAAAA TAGAAAGCTT TTTAAACATA TTATTATTAT 2601 TGGCTTAGAT ATAGTCATTA CCTAGTCAAT TGATTTCTAG TAATTCATAT CATGCATTGC AAAATTGTCG AATGATAAAA TGTATTATTT ATATTTGTAT 2701 AATGGAATAT CAGTACAGGG AAAAAACCCT CACAACATGT TCTTATGATT TAGTCCACAA GGTGATATTC ATACTAATGG TCTATATTAT TAACATCCTA 2801 TCCTCCAAAG TGTACAGACC TATACTACTC TAGAGTGCGG AGCCGAATGG CGGACCTATT AAACACTTTG ACCTAACCCA TTTTTAATAA GTCATTTGCT 2901 ATTATCTTGA AATACTATAT ATAGCAACAA ATTAGTTAGG GCCAGCAGTT GTGATAGGGA GGGAGAGCTA CTGTCCATTA TACACGCGGT GATACGTTAT 3001 CGTACATCGT TACTATGATC TGATTCATAT ATTTATATAT GAATAAGAAC TATAATCCCC AAACGGGTTC AAAGCCTAAC CTACCATACT AATTAAATTC 3101 GTATAGAACT GAGCTTCGTT GTGAATAGTT TGCTGCATTT CATGAAATGT ACATATCGGA TCTCTATTCT TAAATGAATA ACGATTATTT GAGAGAGGAC 3201 ATTTCCCAAG CCCAAAAAAG TGATATAAAA AATATCAAGT ATACACGAAA CAAGAATTGC AAACTGTTTA AAATTAATAT TATATTTGTA TTAATTTACA 3301 ACACCAACAA TATCCCCAGA TTTTGGCATT AATTTGTTTA AAAGATATAA ATTTTTGTAC CGAATACGAT CTATAAATTC GATTAATAAT AGTTATTTGT 3401 AAAACTAGCT CAATAAAATG AAGCATTATT AAACAGAATC AAATGTCTTC TATAAAACAC TCGCCAATCT CGCAACTTCA AAGTATTTTA GTGTTTTTAT 3501 TATTATTTTA TAAATTCAAA TATTCCAATA ACTAATAGTT AATTTATGAA ATGTATAAAG GTAATAATTC TATCATTTCC AACTAAATAT TATAACCTAT 3601 TTCACTGTCC TAAAGTGTAC TTCAGTCAAA TTGTTACTTT CTCATAGAAA GGGGCAATAC GAAAAAGGGA AATTTTGATT TTGAAACTTA CACACACTAG 3701 TACAGCAATA TTTTTTTCTA CACAAATCCT GCCCTGAGCA AATTTTGTTT TGAATTCTAT CAATTAATGG CAATGATATT TTTTCAAAAT ATTTTTTAGT 3801 TTCACACCGG AACGTCGTGT AGGTCGGACC TTACGTTCTG TATTCAGTCT CTGAAAACTA AGAACGTATT TTAAGTGTTG TTTATTAGTT AGTTAAAAAT 3901 CTGCTCTTAC CAAAAACGAT TGTGCTTATC TCCTGTGAAT ATTATCTATA TTAGAAGTGA TTATTATATT CGCAAGACCA CCGATAATGT GAGATGTGAT 4001 TGTGATAAAG GATACTCTAT TTGTTGTACC CCAACTATCC TGGATTACTC GTATATTTCG ATTATTGTGC TTAAAAACTT ATTGTGATAG TGAAATCCCG 
4101 ACAGTGTCCA GTTCTTTTTT GCACACTTTT CCAACTACAA AAAAGGTTAG TGTTTTATTC ATATTTTTTA ACAATAAATA TTTTTCATTT GATCATATCA 4201 GAAATTATTT AAAGAACCTG TTTTTAATTT TATTTAATAA TGTCATCGTT TATTTTTTTA AAATTATTTA CCAAGAATAA AAATTGAATA ATACAGTTTC 4301 ATCTTGAATA AGTTTTTCAT AATGTAAAAT TTATTTTGTA TTCATTACTT TTGTGCTTGA AAACAAGTTT AGCAAAAACA ATATAAACCT AAATTTTTAGA 4401 ATCAGACGAG AATTGCAATG AAATATACCG AACCGGTTTT TAAATAAATA ACCTAAATGT TGAATATCAA TCATATTTGA TTTATTTTAA CTCAATTTAT 4501 AGGAATCATT TCTTTCTAAA ATTAAATGTG AATCATAATA TTACCTATTT TATTATAAAA CTGATTCATT GCAATTTAAT AAATTAATTA TATTTCATAG 4601 TTATATATTC AAATTTTAGG ACACACTCTT TTCTAGAATA AACATGTTTC TAGAGCATGT CGTATTTAATA GACACACTTG GACTATTTGG ATTATTAGCC 4701 TTTTTTGCAGA AATGTGTCCC AACAAACAAT TGCATTCTGC CATTCGACAC GCTCCGCCAC CCTTTGTCCG AACTGCTCTA AATAGGATTC TTTCTTGGAA 4801 TTGCTGCAAA TCCTTTGAAA CGGGTCCTAA AACAAACAAC GCAGGCACAT AAAACTCGGT CCGAATTTCC ATTATGGGAT CGATCGGGCC TCTGGTAGCC 4901 TATCCGGACA AAAGAAAGAA GGCGTATGCA AATAAAGTGC GAATTTATGG GCGAAAGTCG GTGAAAAGTT AATTTCTAGC CGTAAAATCG AAAGGATAAT 5001 AATGGGACGA GTACCTAGAA TTTTACAACA CAGTCCCAAT AAAGCAATAA ACAAAAGTCA ATTGCGAATT ACTGCAAAAG TCAATCTTAA TCATTTTTGGC 5101 AGATCATCAT GATTTACCCA AGGCCATAAA AAATCATITTA CCTACTGTCC ACTATTGGCT CCGCAACTTG GTTCGTAATG ATTTCCCAGA ATTTAGACTC 5201 ATCCTCAATC GGTTGGGTTT TAAACCAGTT TCCCACAGGC AATGGATTTT TAATGCTAAT TATGTCTAAA AATTTCAAAA GGTGTCTTTG CATTTTCTCG 5301 TTGTAAATTA GTATTTTCGA AGAAAAAAAG AACTATCGTA TTTTAAACTG TATATGAGTT AATTTTGATC ACAGAAGATA AGTATTTGGC AATAGTTTTA 5401 ATGCTAATTT CTTCTTAAAA TTCCAAATAA ATCCATTTCC AAAATTCTTA GCTTTTTTTA AACCAGACAT TTCTTATTTC ATTGATAGAC AAAAGATTAT 5501 TTTTAATTAA TTTCGTAAAG AAAAATAATA TTACAAAACT TGTAATTCAA GAAGCGTTGA ATATGGAAAG CCTTTGGGGG TGAGAGAGAT GATTTCGGAA 5601 GGCGCTATTA ATAACATTAC AAATTATTAC CTAATAAATT CAAATGAGCC CATAAAAACT AGACTTTATC TGCTTCATTG GCTTTTGTGC TGGATGCTCG 5701 GATGATAAGA AGGGAGAGAG CGAAACAACT TTTTATGGCC GGATTGGCCA CAAATACAAA AGCTTATTGA CAGGGAAAAA CTGTTGACAA GATAAAGAAG 5801 AGCAAATCAT TGAATTGGAG CAATCAATAA AAATTTAAATG CGTCACGATT GCAATAAATC AAATCCAAAC TGTTGGCAAT TTTACGAATT TTATTAGACC 5901 GTGAAGGAAA TAAAAGTTGG AAAGATCTTC TTCTCCAATT GATCACTCGT TTATAATTAA AAAAGGAACT CTCGAATACA AACAGTTTTA TATTTGTTTT 6001 CAACTTTCCT CCTCTAGTAT TCCTTTGTAA AGGATTCTTC TTTATGGCGC ATAGTAAAAT TGGCAATCGT AAAAACTTTG CAAAACAGTA ACATTCATTT 6101 TAATTCGCAA GTTTAGATTC CAGTCTCCAT TTTATATATT TCGTTTTTTA TATACAAGTA TTTTATGGCG TATAAAATTA AGGGAGTGGT GCTTTTTTAT 6201 GTCCCCTAAA AAAGCGAGCC GTTTTTCTCA CCCCACTTTT ATATGCGCAA ATAAATATAA GGGCAATTTG GCCGTCCCTG TGTTTGTACT CGTATATTTT 6301 GGGGGATTGT ATTTCCAATG CGCGTGCCAC ATATAAAATT TCCACCCCAT TTATAGCACA GCGTATTTTTT TGCATTATAT TCCCTTCTGA CGATGTCGCC 6401 TACTTTTCTC TCCTCGTCGA CATCCTCGTC CTCATCCTCC CATTTTATTA TATTAATGTT TGTACAGCGT TTCCATTTTT ATGGACATTT TTTGTCCATA 6501 TTTTATTTCT TATACCCATA TACGAGTATA CATTTTACAC AACTACTTGT GCCGTTTTGG TGATAAATTT TATTCGCACA CAGATATGGC AAAAAATGGA 6601 ATTAGAGTCC AGTCCAAAGG GGGCACAAAA AACTATGTAC TTTGTGCGAT GCGTCACCAA ACTCCTCAGA GGCTTTCCAT TTTTATCAAC CGTTTTCCTT 6701 GTCGTTTTAA TCTAGTTTCT CTATGCGAAC AGTCTTTTGA GTTAGTTTTA AGCCAGGAAT TAGTAGAATC AAGAAATCAA ACTCTCCCTT TACTGCCAGA 6801 TTTCCAATTC TCGGACGTTA ATTCTAAACA AAATGCTAAT TGGAACCCCT TTTTTTCTATT CTTTCAGTCA AAATGCATAG CTGGGACGTG ATTCCTCAAA 6901 GCAACTACGA CAACAATTGG TATAACAACA ATTACCAGAT GAAAACAGAG CCCCACGATG GGTTCAACGG GCAACAGCCC AATTCTCCGC AGAGCATGGA 7001 CAGCATTCAT CCTGAAACAC ATCACAGTCC TCCAGTTCAA CAGCAACATA TGATGTTCGA CTCGTCAAAT CTTATAAACA CCATGACCCA ACTACACAAC 7101 GTTCAAATGC AGAGACAGAC CCACTTCAAT CCCCTTACTC CTCCGGGCTA TCCAGGCGCT ATGACCCTTC CCCAAAACTC TCAGGCAAAT TCAACACCAT 7201 TTAGAAGCTT CACAAAGGGA CTGGACTCGA TTCCTTTTGG AAATAATGTA TCCAACTTAA CACCAAGTCA CACACCTCCA ATGGACATAA CTCCGCCAAA 7301 GTCACCAAAG TTCAACGGCA AGGAAACCCC TGAAAAGGAC TCTCTAAAGC AGGACCAAAA TCAACTTCTC AAAACCCCAA TCCAGACGAA TGGAAACCAG 7401 CAATCGACAT TCGACTCTGG CGAAGACAGC CACTCAATGC CCGATAGCGA TCTGCTTGAA CCGGTAATCA CCGACGGTGC GGACGTAGAT GACGAAAACG 7501 ATGCTGAAGA GGACGATGAC ATTCGCACTC CGAAAATCAA TTCACACGGT AAAATGAAGA CGTACAAGTG CAAACAGTGT GACTTTATCG CAGTTACAAA 7601 ACTGTCCTTC TGGG

Source: Two PCR products, both amplified from phage Cal-hb ph1. Alignment with cDNA sequences: Positions 3825..4143 correspond to the first exon of the Clogmia hunchback P1 transcript (SEQ19), and positions 6865..7614 correspond to parts of the second exon of the Clogmia hunchback transcript.

\section{SEQ21 Anopheles hunchback, cDNA, partial P1 transcript.}

1 TCAGCAGCAG GACATCGTCG AACCTTTTGG TCTACATTTT CTTCGCCACA TTTGCATCGT GCAACCATTC AGAATGCA

Source: 5' RACE product, amplified from a cDNA template of adult females.

\section{SEQ22 Anopheles hunchback, genomic.}

1 GCGACCGGAA TAGAGAATGG AAACGTGTTA TGAAGGAAGC ACATGGTTTT GAACCAGAAT ACGAAGATAT GTTATAATTT TATGTTGTTC ACTGATAATT 101 ATTAAATTGA ATCATTTCAA GGCATTATTT AAGACTGTTG GTTAAAAAAA TCGGTGATAA TAGTCGTTGA TCTAGAGGTT AGTTTGAATG TTAATAATAA 201 AATAGTTTAG TAAAATGTCG TAGCACAAAT ATACAAGCCA ATTGCAAATT GGCAATAAAT AAAAATAAAT AAATAAATAA ATAAATAAAT AAATAAATAA 301 ATAAATACAT AAATAAATTT TTTCTTTCCT ATTAAACACA AATCTCACAT TATCACATAA AATTACACTT GATTCTTGCA TTAAATTCTA CCTAAAGCCA 401 AAGAAAACTG CTCTGTTCCC CATGCCTTCG GTTATTCGCC CAGCCTAACA ATGGGGGAGG AAAAAAAACC GCTTCCTTAC GGGTCGTTTA CTCCGTCAAC 501 GACTATCCCC AGAATTTTGC TGATTGCTCC GGCTTGGTTA GACGATTTCC TTTCTGGATT TTCTCTGCTT CTTTTGCTAC CACTAGCGGG GTATGAGTTT 601 TCCCTTGACG CAAGTGTGCA ATGTGCGAAG AAAATAAAAA GCCCCGAGG AGTATTCTGT GGCGGCGGTT ACGAGTGGCG AGGCAAATGT CTATCCACTC 701 TTCTTTACAC GGGTACAGCA CACAAAAACA AGGAAGAAAA AGGCCCACTG GGTGGTTAGT GGGATGGGGG CGCACTCTGA TGCTGTTTTT TTTTGTTTCG 801 TGCAGCCAAA ATATTTTCTC CATTTTTCCG TTGATCATAG CGATATGTAT TAAAGCACGT GTGTTAGTGT ATGTGAAAGT GTTTACTTTC ATTTCCCATT 901 TCTTTGGCTA TTTTCCCATT CTTCTTTGCT TCCGAACGCT CCGCTGCAGT GCGTTATCTG CGTTTGTTTA ATCGGGTTTG ATTACAGTGA AATGAAGCTG 1001 ACCGTCCAGC CATTGGGAAG GGCCTAATAG CCAACACTAC TGCGAGGGTG AAATTATGAG TGTAAATAAT CCACACATCT CCCACAAACA CACACTCACG 1101 CGTGTTTCTG ATCGGAAACC ATCATCATCG TCAACATCAT CATGAGAAAA AAGCATCAGT TTCGTACGGG AAAGTACGTA AAATCGTGCC CCATCACGAC 1201 AGCTACGGGA CTCACAAATG CCGGCCACTT TAAAAACCGG GATTGTAGTA TTGCCACACA AGTGTGTTGT TTTTACACGC TGACAAAATA TATTTATTTT 1301 CATTAATTTG TCTCTAGCCG GGCTCGCTGG GCGCGGCAAA GCCCGTAATG CGCCTCGTAA AAACCATCCG GGTGTCCAGG GCGGTATCGT TAAAGTGCAT 1401 TTTAAAAGCA ATTAAAGCCC AGTAGCCGCA GGCGCACTTT TGCGTAGAGC AGCTGGGTCA GAACAATAAC AAGCGGACCT TAGCGACACA GACACACACA 1501 CACCCGGTCG GACGGGCACC AGGGCCCGGT GGACGTGCGC GTCACACGTT GGTTTTTTTC GGGCGAGACT TACACGGCGA ATGAAATGAG CAATCGCTTT 1601 AGCGAATAAT TATGTCTTCA GTAAGTGTGA GCATTTGCAT GAGGCTGATT ATGCTAATAA TGACCGTAAA CATTCCTAAA AGTGAATGAT GTTGTTTGTG 1701 ACGGAATTGG TTTAATACAA AAATGCTTAT TTATTAAGTG TGTCCGGTAA TTGCTGTTAG CATCAAATTT CATTTCGATC CATAATAAAG ATGTCTTTAA 1801 AAGTGTTTAA AATGATTTTA TTTCATTTAT TCATTTTTAA ACCGATGTAC AATCATGTCT AATCGGCAAT TTTATACAGG TATTCCCCGA TATTAGAATA 1901 TTAGCGTAAG TTGAATTTCG AGATTTTCAG TCAAATAATA GCTATAATTT CTAAATTATA ATTAAATTAT AGGTAAGTTT TATAAAATTT TGCTTGAATA 2001 ACTAAACAAG TTATTTGATC TGATTTCAAC TGAAGATTAA AAAACTCAAA GAAATGATAA TATATTTTGC ATTTTACAAT TGATATGTCA AATCAGTACA 2101 ATTTGCTCAA AGAACTGTCA AATTAGGTCA AATAGCGTAT AGCGAAATCG CGTATATCGA GTAAGGCGTA TATCGGATGA TATTAAATAT GACGCCTTAG 2201 TTATTAAATT AAAACAACAA CATGAAATAT TAAATTTGAT TAATTATCAT CTTTTTATAA ACAAATCTCG TAGATAAAAA TCCTAGTCAT GGCACCAATA 2301 ATGCTGTTCA ATTGGCTTTT AATAGACCCA ACATTCTCAT ACGGAAAGTA AAGTGCCATA TAGACATATA TAGCCGAAGA TAACAAACAA AATATAATAA 2401 ATACATATCT TTGCATTAAT TTTTCTCATT TTGATTTTTG AAACTCATTA AATATGACGA CTGGAAATTC ATGTCACTTT GGTGGATTCC GACTATCGTG 2501 TGATAGAGCA TGTAATGAAA TTATAAATGA AATACAGACT CTGATAATTT AGATGTTCAA TCGATTGACA ATTTTCATCA AGAATTCGTG TTCACTTATG 2601 TCTTGTATTT TCAGTGTATT AAGGATTTTT CAAATATTTG ATCGTTAATT ATTCATACGG TGAACATTTT TTTTATTTTA TTATCACGAT TTAGTTATTT 2701 TTGCTTTTAA ACGTTATAGA AAGCATTGAT AGTATGGTTT GCGTGTTCGT TAAAACAATC TGACAATATT GAACGCGTTA CAGGAAAGAA AGCGATGAAA 
2801 TTCTTCATTC GAATAAAAAT TTCATGTTAA GCTTTCATAA ATACTTAATT GTGTTAAGAA ACACGAAACA GACAAACAGA CATTATTTCC TTGCAAAATA 2901 CTTTTAGATG AAAAGAAAAA CTTGATTCAT ATTTCATATT ACTTATTTCA TATGACAGGA AAAAAGAGAC GTATTAATAG CTGCTAAATA ACAGATAAAT 3001 GAATAAATCA ATATCAATGT ATCAAAATAA CAGTGCAATT AAGCGAATAT TTAGGGCAGC AATGCTTCTA GTTGAAAACA TGCTGCTTTA TTGTTTTGCA 3101 ATAAAACAAT CATCAATATT CACTTAAAAT TCATCCAACG CATTTGCCGC TTGCTAGACG CTTAATGCTT CATAAAGTAC GTAACTCGTT CCTCGAGCTG 3201 CTTACGAAAT CGAACCGGCT AAACCACCGA AACGGAGAAA GTTGCCACCT TGCCAATAGA GAGAAGAGCA GCGGCGTCCA CCCAAACCCT CAATCACCCC 3301 AAAAACCACG GTAAGTGATT AGGAGGTGAC GTACGCAGAA AGGTGAAAAA CGGGAACAGG GTTAGTGCAC ACAAACACGG ACGAAACAAA AAAAAAGCAT 3401 AAGCCCTGCA TCAGTACCAC GGTAGCACCA AGTGCACCCC TTTGCCCCAT ATGTTGTCTT GCTAGACGCA CTTTCGATTT CAAATACACA CACACACACA 3501 TATACTTGTC CACGGAAGAC AAAAATCAAT TTTCCTTCTT GTGACCTTCC CTGGGCAAGA CATGCTTCTT GGGTCGTTCC CCGTTCACGC CGAATCGTTC 3601 CCCCGTGCTC AGTCTGTCTC GCTTCACTTC CGAAACTTTA CCTCTGGAAA AGCCCTACCC AGGCCCTGCC CGACCCAAGG ACGGTTACTA TGGACACGGC 3701 GAGAGCCACG GAAGCGAGAG ATAGAAATAG ATCAACGTAG GGCATGAGAC AGAGAGAATG AGAGCTAGCG GCAGAGCTCA GCAACTTACG AGCAGGCGTG 3801 AAAGAGAGCG CGTTGGAATA GGGAAGAGGA AAAAAAAGGT TCTGTTTTCT TCTGTTTCAC CCTCACGCAG TGCGTTGTAG CTACCCCTAG GGATGCATGG 3901 GATGGTTGGT AGTACTGGCA GGTCCCAAAA AATCTCAGAT CAGTTTTTAA ATCGCCAGTG TGCAGTGTGG TAGTCCTTCT GGAAAGCCTG CTGGTCCCGC 4001 ACGGAGTTTT GTCAAGTCGT AAGCGTATGT GTTTTGTACG AGTTCGAGTG TACGTACGCG GAACGTTTTC GGAGCAGAGC AGCAATACAT TGCAGGCCAA 4101 AAGTCCCGGT TGTTACGAAA GTGTTCAGTT GCAGGAGTCG TTTAAAGACG GTGAAACGTT ACACTGTTGT GCTTGTGATT GAAATATGCT ACAAATCGAA 4201 TCGAAGAAAG ATTGTGTTTA ACAAAGTCAA ACATCTTCCT GGAATGTTTG AGTGATTTGC AAAGTGTTGT AAAAAAAAAG AAACTGAAAG TGAAGAGACA 4301 CAAAAACGAG AGACACAATT TCGTCACGGA CCCGTCCCCG TCCCCGTGAT GCTGTAGTCG GCCTGTGGTC AGCAGCAGGA CATCGTCGAA CCTTTTGGTC 4401 TACATTTTCT TCGCCACATT TGCATCGTGC AACCATTCAG GTAAGAATGT CACACAGCAC ACAGAAACCC AATCGGTTGG AGGTCGAATC TCACTTTTAC 4501 AACACAAACT CATGCCGGCA CAAACGTACG AGCCCGAAAG ATGATTGACC TACTTCCACG CCCCCCCGTC CCTGCATCCT TTGGCATTCG AAGGACAACT 4601 CTTTTGTCCT TAAGATTTTA CAGCGCAACA TTGGGCCCCC GCAGTACGGT GGGATGGTGC AATTAGGAAT TGGCAAACTT TATGATGGCT TCCCAAACTT 4701 TGACTTTTCA AAGCTTTCCT CTCCATTGGC ACTCTCCTGT TGTTGGGTAG GTAGGTGACT CAGGGACATC GTCGAGTCTA CCCGTTCGTA TATGGTCTGA 4801 GAAAGTACCC TCGGTACTTT GGGTGAGGCG TATACAAGTC GGGGGCGGTT CTTTGGCCAA CCGAACGGAG TGGGAAAGTC AAATTCGGGG GTCAGCTTTC 4901 GTTTCGGGGT CTCGTTTGCG TTCGGCCCAA GGGTACGGCT GTAAAATCGA TTCAAATTCA GAGAGGGAAA AAGGGGAAGC TTGTAAAAAA ATAAAACTTA 5001 AAAATAACAC ATCTCCAGCA TCCCATGGTG ATTTTGGGAA CGGGGACTTG TGCTTGGGAC GGTTGCAATG CTTGCGAGGG AAAGTGCATT GTTGTCACAA 5101 TTGTTGCCAT CGACCTTACC CTTTACACCG AAACGGGACG CACCATTTGG GGCGATGGCA AAAGGGTCGC AAAGGTTCGC TCTTAGTTCT TGCTACGCTC 5201 GTAAAGTCGA ATGTTTATTT ACGAGCCGTG AGGAAAGTGT TTAGATTTTC GGGATATTTG TGACGTGGGT GACGCATAGA ATGGGAAACA ATCGCTTCCT 5301 CTACGCGTAC TTTCAAAGAA CGAATGGGTA CATGTGGAAA TGGCTGGAAC GGGGAAATGA AATGGCCGTT TGTTGCATTG TAAAAACCGA CAAAAACGAG 5401 GTAGAACCCA GAGTTGTATA GGGTGTAATT GAAATTTTAA TGTTAAAAAC AGACGATAAT GTTTGGAAAT TATTAAAACT GCAAAGCCTC ACAATGTTTC 5501 AAGCTAACAA AACGTTCATT TTTGTTGAAA ATATGTAATT CTTCATCATA CTATTAAAAG AATTGAATTC ATTTCAATGG AATATCATGA GAATATTCCT 5601 AGAATACAGT AAATTAATTC CAATTTTCCT TGAAGTATAC ACTCATTATG TGATGCTTCT CGGCGACGGT ACATAATGAA ACGGCTCCCC CACAAAATAT 5701 AACGACAAAC AATAGCGTTT TGTGGAGGAG GATGAGGCAA AAAAAGCCAA AACCACAAAG AAATATTCTG GATTACGAGA ACCCTCCCAA ATATACAGAA 5801 AAGAGAAGAA GGAAGAAAAA AAACGAACAC ACAAACCTTC TTCTGTCGCA ACACACAAAA CGCAACTTTC CTGCCATGCC GGGTGGGTTA TCGATTTTCC 5901 AAACCCACCA ACCAAGCATT GAGCGTTGTG AGAGGAGGCA AAAAAACGGC AGGCAAAAAA GGAATAAAAG ATTCCGCAAA AATCCTCCAA GACAAAGGAG 6001 GAACGTACGC ATTTTCCACC CCAACGAAGG AGAAGGTAAA AGGGGGGAGG GTGGTGTAGG AGAAGAAGAA AGAAGAAAAA CTCCTTCACG CAAGAGGGGA 6101 CAAACAAAGC CGGCAAAATG GGAAAAATGC TGCTCGGTTG TTGTGTTTTG GTTTCGGTTT CCATTCCAAG GGAAATCGTT CGTTGCTAAT GGAAGAAGAA 6201 GAAGAAGTAG CCCCCAAAAA AACTCTCTTC TCTCGTACTA AACGAGCACA GATACATTCT TGCAAGCAAC ACACACACAT AACACTAGGA GAAGCCGGAA 6301 AATAGTAAAA CACACAGCGC GTTCAATTAT CGTTTTTCGG GATGGAATAG GGGGGAGGCT TGTGGTAAAG ATCGTTCCCA AACGCAGGGA AATAAAAAGC 6401 TGCGATGAAA ATAAAAATAA TCACCCACAC ACACACACAT ACACGCGCAT GAGTGTGAGC AGCAGCACAT TTTGCCCCCT TTTTTGTCTT CTCGGGACAT 6501 TTAATTTGGC TGTGGGGAGG ACTTCTCACC CTCGCAAACA CATATACTTT TCACCGACCC TCTATCACTG TGTATGTGTG CGTGTTTATA TACCGAGGGT 6601 GTAGCAACAG CAAAAAAAAA ACCGTGTGGC ATCGCTGTGT GAGTGAATCG CACTCGCGAG GGGAGAAAGT GAAACACACA ACCATCCATT ACGGTTACTA 6701 AAGGGCTTGC CTACTCACAC AAATACGTAC ACCGTTTTAA GGGGAGGATA AAACGAAAAA AACATGAACA CAGCATGCCA TGTTTTACTG ATGGTTTTGT 6801 TTCTTTTCTC TTTTCCCCCA TTTTTGCAGA ATGCAGAACT TTGACTCCGT AATGGCGATG GCGGCAGCAA CACAACAACA GTCACAGCAG CAGCAGCAAA 6901 CGGCAGATCA ACAGCAGCAA ACGGCAACGC CAAACGGTTG GTTCGATTTA ACGATCAAAT CCGAACCACT CGACTACCAT CCACATCATC ATCATCCGCT 7001 GCAGCAGCAT CACCATCATC ACCTGCACCA TCATCGCCCG GGCGGAGTGC AGCAGGACAC GACGACGCAC CTCGCGAGCG ATTCACACTC CAACCCAACC 7101 TCCCCGCAGA GCGTGGACAG TATGGATTCA CTCAGTGGTA AGAGTGGATT TTTTGAGGCA AAAGGAGCAA ACAGTCTCAT GTCGGGCGGC TACAACCCTC 7201 TCGGCTTTAA TCCACTCACA CCGCCTGGCT ATTCGGGGCT GCTGATCCCA CCACCACCAG CACAGCTCGC CGCTCAGCAG CAGCAGCAGC ACCAACAGCA 7301 CCAGCAACGC CTAGCAACAC CCAACCGAAT GTACGGCAAT GGTACGGGCA AGGTGGAGCT GCCAACCTCC CTAACGCCGA CCCACACCCC ACCGATGGAC 7401 GTGACGCCAC CAAAGTCCCC CAAGGAGTCG CTCGAAACAC CCACCAAAGA GGAGGACGCC GGCTCGGACT GTGAGGATGG CTCGTACGAT GGCAGCGAGG 7501 ACGAGGACGG CATCCGCAAG CCGAAGGTGA ACTCGCACGG TCAGGTGAAG AAGTTCCGCT GCAAGCAGTG CGAGTTTGTC GCGGTAACGA AGCTCAGCTT 7601 CTGGGAGCAT ACGCGCAGCC ACATCAAGCC GGAAAAGATG CTCACCTGCC CAAAGTGTCC GTTCGTGACG GAGTACAAGC ACCATCTCGA GTACCATCTG 7701 CGCAACCACC AGCGCTCCAA GCCGTTCCAG TGTCCGAAGT GTAGCTACAG CTGCGTCAAC AAATCAATGC TGAACTCGCA CATGAAGTCG CACTCGAACG 7801 TGTTCCAGTA CCGGTGTGCG GATTGTAACT ACGCCACCAA GTACTGCCAC TCGCTCAAGC TGCATCTGCG CAAGTATGCG CACAAGCCGG ACGTGGTGCT 7901 GAACCTGGAC GGTACACCGA ACCCGCTACC GATCATCGAT GTGTACGGGA CACGTCGTGG ACCGAAAGCG AAACCGCAGA AGAACGCCGA TAAGCATCTG 8001 CTCAAGCAAG AACAGGCTCA GCAGGAGCAG CAATCTCAAC CACTCAAACA GCAAGAATTG ACCAACTCCG GAGATCAATC GCCCGCACCA CTAACGCCAC 8101 TAAGTCAGCA GGGCATGGCA ACGGCACCGA CGACTCCGTT CACCCCTCCC GGAAGTGGCC AAACGAATGG GCTGATGCGC AACCTCTTCC CACACCAGCT 8201 CTTCCCGAAC CCGCTCGCGC ACATGTTCAA GAGTGCGGCG GCCAACGGCA GTCTGCCACT GTTCCCGTAC CTGAACCTCA ACTTCCAGAT GTTTGCCGAC 8301 CAGCAGGCCG GCTTGCAGCA GCAGCAGCAG CAACTCTCCC CCCGCACCTT CCAGCAGAAC CTGATGAACC AGCTGGCGGG CGAAAAGCTG AACGGCAGTG 8401 CAACGAGCAG CGGCTCCGAC CAGGACAATG ATCTTTCGGT GCGGACCAGC TCCAGCGGCT CCACCCTGGG CGAGGAGCTG CTGAAGCACA TTACGGCGGC 8501 GGCGGCTGAC AGTTCGGCAG CGGTGGGACG CAATGCGCTC TCCCAGGAGG ACAGTGAATC GGAGACGACC CAGGCTACAC CGCTGCAGGC ATCATTCGAT 8601 GAGTTCCCGG CATCGGTATC GTCCAATCCG CGGACACCTT CGACCACCGG TAAGGGTGAg CGGAGTCGCC GTAAGGGACG TGCCTTTAAG CTGGAGCGGC 8701 TTACAGAACC GGCCACTTCG TCGGGTGGCT CAAACGAAAT GGACACACAC TACGAGCACG CTGAGGAAGC GGCGGTCAGT ACACCGGTGA CAGTAGCTGT 8801 CACTCGAGAA TCCCCTGCGC CGCCCAAGGA ACGTTCACTC GAGTGCAAGT ACTGCGACAT TGCGTTCCGG GACGATGTGC TGTACACGAT CCACATGGGC 8901 TATCACGGGT ACGACGACGT GTTCAAGTGC AACATGTGCG GCGAGAAGA TGACGATCGC ATCGGCTTCT TCCTGCACAT TGCCCGCAAG GCGCACTGAT 9001 CCGCTGATAG GGGACGCCCG AGATGGTGGC GGCGTACCGA ATCGGGGATG AATGCGCATG CGGGACACAC TACCTGATAA GTATTTATTT ATTGTAAGAG 9101 GGACAGGCGA AGACAACGTG TTGGTTGACC CTTATTTCCC GATGAGTTTG TATATGCATA CATAAATAAT TTCGCAATGG CAGAAGCTAG TCGGCAGAGA 9201 ACGTTTTCAG AACGTTAGTG GGAAGAAGAG GCAGTTTGGG TTTTAGTGGT TTGTCTGTAA AAAAGCAACG CAATTTGATC CATTTACACA CAAACTAAAG 9301 TACCCGAAAA CAATCCAAAG ACTGAGCAGA GGAAAAAGGA AGGCAAAACG CATGTAGAAA GCAAGAAGAT AGAGGAAGAG ACAGTAATGC AGGAAGTAAA 9401 AAGTTACTAT AGTAAGTAAG ACGGCACAGT ACGAGCAAAA GAATCAAAGA TATTCGTAGG ATGAGATGGT TTGGGCGAAG CTCCTCGGAT TTTCGCCCAC 9501 CCCGTTTTTT GCCTCGCTAG TGGTTAAGAG ATTGTTACGA ACTTCTTAGC GCGTAGAACA TTAGGATTAT TTATACTGGT TGACGGTGCA CACAATAAAG 9601 AACACTAAAT TCATCTAAAT TTTCGGGCCT TATGTTTTCT TTTTGAAAAT ATACTTTTGG TATAACTTTC TCCATCACAC AGAAAGCTAC AGCTATTTCT 9701 CAGTTTTCTG TAGTTGAATC CAAGGAGGCA TTGCAAAAGC TTTTGCTAAG TTGTTCGTTA AATTTTACTT CCACTCCTCT TCAAGAAATC CAATCCATTA 9801 AAAAAACTGA AAATTGAAAT CATTTTCCTC ATTTTCATTA ATTCCTGTAA AGATCTTTAG CGTCCTTTAA AGTTCCTAGC ATCTTTCGTA AGGTTAGTTG 9901 CACTAAAAAC GATCTTTACA GCGATCTTTA CAGCTAATTA GCACCACGCT CAAGTAGAAC GCTTATGATG TCAATCGGGG TGTCTTCCGA TTGGGATCAT

Source: GenBank entry AAAB01008979 (563001..573000). Alignment with cDNA sequences: Positions 4367..4440 correspond to the first exon of the Anopheles hunchback P1 transcript (SEQ21), and positions $6830 . .6835$ correspond to parts of the second exon of the Anopheles hunchback transcript. One putative polyadenylation signal was found in the selected part of the genomic sequence (9594..9599). 


\section{Bibliography}

Altschul, S., Boguski, M., Gish, W. and Wootton, J. (1994). Issues in searching molecular sequence databases. Nat Genet 6, 119-29.

Altschul, S., Madden, T., Schäffer, A., Zhang, J., Zhang, Z., Miller, W. and Lipman, D. (1997). Gapped BLAST and PSI-BLAST: a new generation of protein database search programs. Nucleic Acids Res 25, 3389402.

Anderson, D. (1966). The comparative embryology of the Diptera. Annu Rev Entomol 11, 23-46.

Anderson, D. (1972). The development of holometabolous insects. In Developmental systems: insects, (ed. S. Counce and C. Waddington), pp. 165-242. London, New York: Academic Press.

Andres, A. and Thummel, C. (1994). Methods for quantitative analysis of transcription in larvae and prepupae. Methods Cell Biol 44, 565-73.

Arora, K., Levine, M. and O'Connor, M. (1994). The screw gene encodes a ubiquitously expressed member of the TGF-beta family required for specification of dorsal cell fates in the Drosophila embryo. Genes Dev $\mathbf{8}$, 2588-601.

Ashe, H. (2005). BMP signalling: synergy and feedback create a step gradient. Curr Biol 15, R375-7.

Ashraf, S., Hu, X., Roote, J. and Ip, Y. (1999). The mesoderm determinant snail collaborates with related zincfinger proteins to control Drosophila neurogenesis. EMBO J 18, 6426-38.

Benson, D., Karsch-Mizrachi, I., Lipman, D., Ostell, J. and Wheeler, D. (2006). GenBank. Nucleic Acids Res 34, D16-20.

Berleth, T., Burri, M., Thoma, G., Bopp, D., Richstein, S., Frigerio, G., Noll, M. and Nüsslein-Volhard, C. (1988). The role of localization of bicoid RNA in organizing the anterior pattern of the Drosophila embryo. EMBO J 7, 1749-56.

Birnstiel, M., Busslinger, M. and Strub, K. (1985). Transcription termination and 3' processing: the end is in site! Cell 41, 349-59.

Boer, P. and Hickey, D. (1986). The alpha-amylase gene in Drosophila melanogaster: nucleotide sequence, gene structure and expression motifs. Nucleic Acids Res 14, 8399-411.

Bonneton, F., Shaw, P., Fazakerley, C., Shi, M. and Dover, G. (1997). Comparison of bicoid-dependent regulation of hunchback between Musca domestica and Drosophila melanogaster. Mech Dev 66, 143-56.

Boulay, J., Dennefeld, C. and Alberga, A. (1987). The Drosophila developmental gene snail encodes a protein with nucleic acid binding fingers. Nature 330, 395-8.

Brady, G. and Iscove, N. (1993). Construction of cDNA libraries from single cells. Methods Enzymol 225, 61123.

Bucher, G., Scholten, J. and Klingler, M. (2002). Parental RNAi in Tribolium (Coleoptera). Curr Biol 12, R856.

Bullock, S., Stauber, M., Prell, A., Hughes, J., Ish-Horowicz, D. and Schmidt-Ott, U. (2004). Differential cytoplasmic mRNA localisation adjusts pair-rule transcription factor activity to cytoarchitecture in dipteran evolution. Development 131, 4251-61.

Calvo, E., Walter, M., Adelman, Z., Jimenez, A., Onal, S., Marinotti, O. and James, A. (2005). Nanos (nos) genes of the vector mosquitoes, Anopheles gambiae, Anopheles stephensi and Aedes aegypti. Insect Biochem Mol Biol 35, 789-98.

Campos-Ortega, J. and Hartenstein, V. (1997). The embryonic development of Drosophila melanogaster. Berlin, Heidelberg, New York: Springer Verlag. 
Carney, G. and Bowen, N. (2004). p24 proteins, intracellular trafficking, and behavior: Drosophila melanogaster provides insights and opportunities. Biol Cell 96, 271-8.

Casanova, J. and Struhl, G. (1989). Localized surface activity of torso, a receptor tyrosine kinase, specifies terminal body pattern in Drosophila. Genes Dev 3, 2025-38.

Cha, B., Koppetsch, B. and Theurkauf, W. (2001). In vivo analysis of Drosophila bicoid mRNA localization reveals a novel microtubule-dependent axis specification pathway. Cell 106, 35-46.

Collins, K. and Wiegmann, B. (2002). Phylogenetic relationships of the lower Cyclorrhapha (Diptera: Brachycera) based on 28S rDNA sequences. Insect Syst Evol 33, 445-56.

Copf, T., Schröder, R. and Averof, M. (2004). Ancestral role of caudal genes in axis elongation and segmentation. Proc Natl Acad Sci U S A 101, 17711-5.

Crauk, O. and Dostatni, N. (2005). Bicoid determines sharp and precise target gene expression in the Drosophila embryo. Curr Biol 15, 1888-98.

Crevel, G. and Cotterill, S. (1995). DF 31, a sperm decondensation factor from Drosophila melanogaster: purification and characterization. EMBO J 14, 1711-7.

Crevel, G., Huikeshoven, H. and Cotterill, S. (2001). Df31 is a novel nuclear protein involved in chromatin structure in Drosophila melanogaster. J Cell Sci 114, 37-47.

Cummings, L., Riley, L., Black, L., Souvorov, A., Resenchuk, S., Dondoshansky, I. and Tatusova, T. (2002). Genomic BLAST: custom-defined virtual databases for complete and unfinished genomes. FEMS Microbiol Lett 216, 133-8.

Curtis, D., Apfeld, J. and Lehmann, R. (1995). nanos is an evolutionarily conserved organizer of anteriorposterior polarity. Development 121, 1899-910.

Dave, V., Zhao, C., Yang, F., Tung, C. and Ma, J. (2000). Reprogrammable recognition codes in bicoid homeodomain-DNA interaction. Mol Cell Biol 20, 7673-84.

Dearden, P. and Akam, M. (1999). Developmental evolution: Axial patterning in insects. Curr Biol 9, R591-4.

Doyle, H., Harding, K., Hoey, T. and Levine, M. (1986). Transcripts encoded by a homoeo box gene are restricted to dorsal tissues of Drosophila embryos. Nature 323, 76-9.

Driever, W. and Nüsslein-Volhard, C. (1988). A gradient of bicoid protein in Drosophila embryos. Cell 54, 8393.

Driever, W. and Nüsslein-Volhard, C. (1989). The bicoid protein is a positive regulator of hunchback transcription in the early Drosophila embryo. Nature 337, 138-43.

Driever, W., Thoma, G. and Nüsslein-Volhard, C. (1989). Determination of spatial domains of zygotic gene expression in the Drosophila embryo by the affinity of binding sites for the bicoid morphogen. Nature 340, 363-7.

Drysdale, R., Crosby, M. and Consortium, F. (2005). FlyBase: genes and gene models. Nucleic Acids Res 33, D390-5.

Dulac, C. and Axel, R. (1995). A novel family of genes encoding putative pheromone receptors in mammals. Cell 83, 195-206.

Dunham, I., Dewar, K., Kim, U.-J. and Ross, M. (1997). Bacterial Cloning Systems. In Genome Analysis: A Laboratory Manual, (ed. B. Birren E. Green and S. Klapholz): Cold Spring Harbor Laboratory Press.

Elbetieha, A. and Kalthoff, K. (1988). Anterior determinants in embryos of Chironomus samoensis: characterization by rescue bioassay. Development 104, 61-75.

Ephrussi, A., Dickinson, L. and Lehmann, R. (1991). Oskar organizes the germ plasm and directs localization of the posterior determinant nanos. Cell 66, 37-50.

Ephrussi, A. and Lehmann, R. (1992). Induction of germ cell formation by oskar. Nature 358, 387-92. 
Falciani, F., Hausdorf, B., Schröder, R., Akam, M., Tautz, D., Denell, R. and Brown, S. (1996). Class 3 Hox genes in insects and the origin of zen. Proc Natl Acad Sci U S A 93, 8479-84.

Ferguson, E. and Anderson, K. (1992). Decapentaplegic acts as a morphogen to organize dorsal-ventral pattern in the Drosophila embryo. Cell 71, 451-61.

Finkelstein, R., Smouse, D., Capaci, T., Spradling, A. and Perrimon, N. (1990). The orthodenticle gene encodes a novel homeo domain protein involved in the development of the Drosophila nervous system and ocellar visual structures. Genes Dev 4, 1516-27.

Fleig, R. and Sander, K. (1988). Honeybee morphogenesis: embryonic cell movements that shape the larval body. Development 103, 525-34.

Foe, V. and Alberts, B. (1983). Studies of nuclear and cytoplasmic behaviour during the five mitotic cycles that precede gastrulation in Drosophila embryogenesis. J Cell Sci 61, 31-70.

Frohnhöfer, H. and Nüsslein-Volhard, C. (1986). Organization of anterior pattern in the Drosophila embryo by the maternal gene bicoid. Nature 324, 120-5.

Gabay, L., Seger, R. and Shilo, B. (1997). MAP kinase in situ activation atlas during Drosophila embryogenesis. Development 124, 3535-41.

Galindo, K. and Smith, D. (2001). A large family of divergent Drosophila odorant-binding proteins expressed in gustatory and olfactory sensilla. Genetics 159, 1059-72.

Gans, M., Audit, C. and Masson, M. (1975). Isolation and characterization of sex-linked female-sterile mutants in Drosophila melanogaster. Genetics 81, 683-704.

Gavis, E. and Lehmann, R. (1992). Localization of nanos RNA controls embryonic polarity. Cell 71, 301-13.

Gehring, W., Affolter, M. and Bürglin, T. (1994). Homeodomain proteins. Annu Rev Biochem 63, 487-526.

Goltsev, Y., Hsiong, W., Lanzaro, G. and Levine, M. (2004a). Different combinations of gap repressors for common stripes in Anopheles and Drosophila embryos. Dev Biol 275, 435-46.

Goltsev, Y., Peterson, B., Hsiong, W., Lanzaro, G. and Levine, M. (2004b). Evolution of gene expression in Diptera. Distinct features of patterning gene expression in Anopheles gambiae early embryo. A Dros Res Conf 45 .

Gregor, T., Bialek, W., de Ruyter van Steveninck, R., Tank, D. and Wieschaus, E. (2005). Diffusion and scaling during early embryonic pattern formation. Proc Natl Acad Sci U S A 102, 18403-7.

Grimaldi, D. and Engel, M. (2005). Evolution of the insects. New York: Cambridge University Press.

Grosskortenhaus, R., Pearson, B., Marusich, A. and Doe, C. (2005). Regulation of temporal identity transitions in Drosophila neuroblasts. Dev Cell 8, 193-202.

Hancock, J., Shaw, P., Bonneton, F. and Dover, G. (1999). High sequence turnover in the regulatory regions of the developmental gene hunchback in insects. Mol Biol Evol 16, 253-65.

Handel, K., Grünfelder, C., Roth, S. and Sander, K. (2000). Tribolium embryogenesis: a SEM study of cell shapes and movements from blastoderm to serosal closure. Dev Genes Evol 210, 167-79.

Hanes, S. and Brent, R. (1989). DNA specificity of the bicoid activator protein is determined by homeodomain recognition helix residue 9 . Cell 57, 1275-83.

Hanes, S., Riddihough, G., Ish-Horowicz, D. and Brent, R. (1994). Specific DNA recognition and intersite spacing are critical for action of the bicoid morphogen. Mol Cell Biol 14, 3364-75.

Holt, R. Subramanian, G. Halpern, A. Sutton, G. Charlab, R. Nusskern, D. Wincker, P. Clark, A. Ribeiro, J. Wides, R. et al. (2002). The genome sequence of the malaria mosquito Anopheles gambiae. Science 298, 129-49.

Houchmandzadeh, B., Wieschaus, E. and Leibler, S. (2002). Establishment of developmental precision and proportions in the early Drosophila embryo. Nature 415, 798-802. 
Hülskamp, M., Lukowitz, W., Beermann, A., Glaser, G. and Tautz, D. (1994). Differential regulation of target genes by different alleles of the segmentation gene hunchback in Drosophila. Genetics 138, 125-34.

Hülskamp, M., Pfeifle, C. and Tautz, D. (1990). A morphogenetic gradient of hunchback protein organizes the expression of the gap genes Krüppel and knirps in the early Drosophila embryo. Nature 346, 577-80.

Hülskamp, M., Schröder, C., Pfeifle, C., Jäckle, H. and Tautz, D. (1989). Posterior segmentation of the Drosophila embryo in the absence of a maternal posterior organizer gene. Nature 338, 629-32.

Irish, V., Lehmann, R. and Akam, M. (1989). The Drosophila posterior-group gene nanos functions by repressing hunchback activity. Nature 338, 646-8.

Isshiki, T., Pearson, B., Holbrook, S. and Doe, C. (2001). Drosophila neuroblasts sequentially express transcription factors which specify the temporal identity of their neuronal progeny. Cell 106, 511-21.

Jiménez, G., Guichet, A., Ephrussi, A. and Casanova, J. (2000). Relief of gene repression by torso RTK signaling: role of capicua in Drosophila terminal and dorsoventral patterning. Genes Dev 14, 224-31.

Johannsen, O. and Butt, F. (1941). Embryology of insects and myriapods. New York: McGraw-Hill Book Company, Inc.

Jürgens, G., Wieschaus, E., Nüsslein-Volhard, C. and Kluding, H. (1984). Mutations affecting the pattern of the larval cuticle in Drosophila melanogaster: II. Zygotic loci on the third chromosome. Roux's Arch Dev Biol 193, 283-95.

Kalthoff, K. (1979). Analysis of a morphogenetic determinant in an insect embryo (Smittia spec., Chironomidae, Diptera). In Determinants of spatial organization, (ed. S. Subtelny and I. Konigsberg), pp. 79-126. New York: Academic Press.

Kalthoff, K. (1983). Cytoplasmic determinants in dipteran eggs. In Time, space, and pattern in embryonic development, (ed. W. Jeffery and R. Raff), pp. 313-348. New York: Alan R Liss, Inc.

Kalthoff, K. and Sander, K. (1968). Der Entwicklungsgang der Mißbildung "Doppelabdomen" im partiell UVbestrahlten Ei von Smittia parthenogenetica (Dipt., Chironomidae). Wilhelm Roux' Arch 161, 129-46.

Kambadur, R., Koizumi, K., Stivers, C., Nagle, J., Poole, S. and Odenwald, W. (1998). Regulation of POU genes by castor and hunchback establishes layered compartments in the Drosophila CNS. Genes Dev 12, 24660.

Kandler-Singer, I. and Kalthoff, K. (1976). RNase sensitivity of an anterior morphogenetic determinant in an insect egg (Smittia sp., Chironomidae, Diptera). Proc Natl Acad Sci U S A 73, 3739-43.

Karpen, G. and Spradling, A. (1992). Analysis of subtelomeric heterochromatin in the Drosophila minichromosome Dp1187 by single P element insertional mutagenesis. Genetics 132, 737-53.

Kim-Ha, J., Smith, J. and Macdonald, P. (1991). oskar mRNA is localized to the posterior pole of the Drosophila oocyte. Cell 66, 23-35.

Klingler, M., Soong, J., Butler, B. and Gergen, J. (1996). Disperse versus compact elements for the regulation of runt stripes in Drosophila. Dev Biol 177, 73-84.

Kosman, D., Mizutani, C., Lemons, D., Cox, W., McGinnis, W. and Bier, E. (2004). Multiplex detection of RNA expression in Drosophila embryos. Science 305, 846.

Kramer, P. (2000). cDNA library construction from single cells. Current Protocols In Neuroscience.

Lehmann, R. and Nüsslein-Volhard, C. (1986). Abdominal segmentation, pole cell formation, and embryonic polarity require the localized activity of oskar, a maternal gene in Drosophila. Cell 47, 141-52.

Lehmann, R. and Nüsslein-Volhard, C. (1987a). hunchback, a gene required for segmentation of an anterior and posterior region of the Drosophila embryo. Dev Biol 119, 402-17.

Lehmann, R. and Nüsslein-Volhard, C. (1987b). Involvement of the pumilio gene in the transport of an abdominal signal in the Drosophila embryo. Nature 329, 167-70. 
Lehmann, R. and Nüsslein-Volhard, C. (1991). The maternal gene nanos has a central role in posterior pattern formation of the Drosophila embryo. Development 112, 679-91.

Lehmann, R. and Tautz, D. (1994). In situ hybridization to RNA. Methods Cell Biol 44, 575-98.

Lewis, E. (1978). A gene complex controlling segmentation in Drosophila. Nature 276, 565-70.

Li, Y., Brown, S., Hausdorf, B., Tautz, D., Denell, R. and Finkelstein, R. (1996). Two orthodenticle-related genes in the short-germ beetle Tribolium castaneum. Dev Genes Evol 206, 35-45.

Liang, Z. and Biggin, M. (1998). Eve and ftz regulate a wide array of genes in blastoderm embryos: the selector homeoproteins directly or indirectly regulate most genes in Drosophila. Development 125, 4471-82.

Liu, P. and Kaufman, T. (2005). Short and long germ segmentation: unanswered questions in the evolution of a developmental mode. Evol Dev 7, 629-46.

Liu, Y. and Montell, D. (2001). Jing: a downstream target of slbo required for developmental control of border cell migration. Development 128, 321-30.

Lukowitz, W., Schröder, C., Glaser, G., Hülskamp, M. and Tautz, D. (1994). Regulatory and coding regions of the segmentation gene hunchback are functionally conserved between Drosophila virilis and Drosophila melanogaster. Mech Dev 45, 105-15.

Lynch, J., Brent, A., Leaf, D., Pultz, M. and Desplan, C. (2006). Localized maternal orthodenticle patterns anterior and posterior in the long germ wasp Nasonia. Nature 439, 728-32.

MacDonald, P. (1990). bicoid mRNA localization signal: phylogenetic conservation of function and RNA secondary structure. Development 110, 161-71.

Macdonald, P. (1992). The Drosophila pumilio gene: an unusually long transcription unit and an unusual protein. Development 114, 221-32.

Macdonald, P. and Struhl, G. (1986). A molecular gradient in early Drosophila embryos and its role in specifying the body pattern. Nature 324, 537-45.

Marcey, D., Watkins, W. and Hazelrigg, T. (1991). The temporal and spatial distribution pattern of maternal exuperantia protein: evidence for a role in establishment but not maintenance of bicoid mRNA localization. EMBO J 10, 4259-66.

Margolis, J., Borowsky, M., Shim, C. and Posakony, J. (1994). A small region surrounding the distal promoter of the hunchback gene directs maternal expression. Dev Biol 163, 381-8.

Margolis, J., Borowsky, M., Steingrímsson, E., Shim, C., Lengyel, J. and Posakony, J. (1995). Posterior stripe expression of hunchback is driven from two promoters by a common enhancer element. Development 121, 3067-77.

Markstein, M., Markstein, P., Markstein, V. and Levine, M. (2002). Genome-wide analysis of clustered Dorsal binding sites identifies putative target genes in the Drosophila embryo. Proc Natl Acad Sci U S A 99, 7638.

McAlpine, J. (1989). Phylogeny and classification of the Muscomorpha. In Manual of Nearctic Diptera, vol. 3 (ed. J. McAlpine and D. Wood), pp. 1397-1518. Ottawa, Canada: Agriculture Canada Monograph.

McGregor, A. (2005). How to get ahead: the origin, evolution and function of bicoid. Bioessays 27, 904-13.

McGregor, A., Shaw, P. and Dover, G. (2001a). Sequence and expression of the hunchback gene in Lucilia sericata: a comparison with other Dipterans. Dev Genes Evol 211, 315-8.

McGregor, A., Shaw, P., Hancock, J., Bopp, D., Hediger, M., Wratten, N. and Dover, G. (2001b). Rapid restructuring of bicoid-dependent hunchback promoters within and between Dipteran species: implications for molecular coevolution. Evol Dev 3, 397-407. 
Mlodzik, M., Fjose, A. and Gehring, W. (1985). Isolation of caudal, a Drosophila homeo box-containing gene with maternal expression, whose transcripts form a concentration gradient at the pre-blastoderm stage. $E M B O$ J 4, 2961-2969.

Mlodzik, M. and Gehring, W. (1987a). Expression of the caudal gene in the germ line of Drosophila: formation of an RNA and protein gradient during early embryogenesis. Cell 48, 465-78.

Mlodzik, M. and Gehring, W. (1987b). Hierarchy of the genetic interactions that specify the anteroposterior segmentation pattern of the Drosophila embryo as monitored by caudal protein expression. Development 101, 421-35.

Mohler, J. (1977). Developmental genetics of the Drosophila egg. I. Identification of 59 sex-linked cistrons with maternal effects on embryonic development. Genetics 85, 259-72.

Moses, K., Ellis, M. and Rubin, G. (1989). The glass gene encodes a zinc-finger protein required by Drosophila photoreceptor cells. Nature 340, 531-6.

Moulton, J. and Wiegmann, B. (2004). Evolution and phylogenetic utility of CAD (rudimentary) among Mesozoic-aged Eremoneuran Diptera (Insecta). Mol Phylogenet Evol 31, 363-78.

Moussian, B. and Roth, S. (2005). Dorsoventral axis formation in the Drosophila embryo--shaping and transducing a morphogen gradient. Curr Biol 15, R887-99.

Murata, Y. and Wharton, R. (1995). Binding of pumilio to maternal hunchback mRNA is required for posterior patterning in Drosophila embryos. Cell 80, 747-56.

Niessing, D., Driever, W., Sprenger, F., Taubert, H., Jäckle, H. and Rivera-Pomar, R. (2000). Homeodomain position 54 specifies transcriptional versus translational control by Bicoid. Mol Cell 5, 395-401.

Novotny, T., Eiselt, R. and Urban, J. (2002). Hunchback is required for the specification of the early sublineage of neuroblast 7-3 in the Drosophila central nervous system. Development 129, 1027-36.

Nüsslein-Volhard, C. (1979). Maternal effect mutations that alter the spatial coordinates of the embryo of Drosophila melanogaster. In Determinants of Spatial Organisation., (ed. S. Subtelney and I. Konigsberg), pp. 185-211. New York: Academic Press.

Nüsslein-Volhard, C., Frohnhöfer, H. and Lehmann, R. (1987). Determination of anteroposterior polarity in Drosophila. Science 238, 1675-81.

Nüsslein-Volhard, C. and Wieschaus, E. (1980). Mutations affecting segment number and polarity in Drosophila. Nature 287, 795 - 801.

Nüsslein-Volhard, C., Wieschaus, E. and Kluding, H. (1984). Mutations affecting the pattern of the larval cuticle in Drosophila melanogaster: I. Zygotic loci on the second chromosome. Roux's Arch Dev Biol 193, 267282.

Orr, H. (2005). The genetic theory of adaptation: a brief history. Nat Rev Genet 6, 119-27.

Padgett, R., St Johnston, R. and Gelbart, W. (1987). A transcript from a Drosophila pattern gene predicts a protein homologous to the transforming growth factor-beta family. Nature 325, 81-4.

Pankratz, M. and Jäckle, H. (1993). Blastoderm segmentation. In The development of Drosophila melanogaster, vol. 1 (ed. M. Bate and A. Martinez-Arias), pp. 467-516. Cold Spring Harbor, New York: Cold Spring Harbor Laboratory Press.

Paroush, Z., Wainwright, S. and Ish-Horowicz, D. (1997). Torso signalling regulates terminal patterning in Drosophila by antagonising Groucho-mediated repression. Development 124, 3827-34.

Patel, N., Hayward, D., Lall, S., Pirkl, N., DiPietro, D. and Ball, E. (2001). Grasshopper hunchback expression reveals conserved and novel aspects of axis formation and segmentation. Development 128, 3459-72.

Perondini, A., Gutzeit, H. and Sander, K. (1987). Double abdomen induction by UV in Bradysia tritici (syn. Sciara ocellaris, Sciaridae): sensitive stages and conditions for photoreversal. Roux's Arch Dev Biol 196, 26872. 
Perrimon, N., Mohler, D., Engstrom, L. and Mahowald, A. (1986). X-linked female-sterile loci in Drosophila melanogaster. Genetics 113, 695-712.

Pignoni, F., Baldarelli, R., Steingrímsson, E., Diaz, R., Patapoutian, A., Merriam, J. and Lengyel, J. (1990). The Drosophila gene tailless is expressed at the embryonic termini and is a member of the steroid receptor superfamily. Cell 62, 151-63.

Pultz, M., Westendorf, L., Gale, S., Hawkins, K., Lynch, J., Pitt, J., Reeves, N., Yao, J., Small, S., Desplan, C. et al. (2005). A major role for zygotic hunchback in patterning the Nasonia embryo. Development 132, 3705-15.

Raff, R. (1996). The shape of life: Genes, development, and the evolution of animal form. Chicago: The University of Chicago Press.

Rafiqi, A. M., Lemke, S., Ferguson, S., Stauber, M. and Schmidt-Ott, U. (in preparation).

Raftery, L. and Sutherland, D. (2003). Gradients and thresholds: BMP response gradients unveiled in Drosophila embryos. Trends Genet 19, 701-8.

Ray, R., Arora, K., Nüsslein-Volhard, C. and Gelbart, W. (1991). The control of cell fate along the dorsalventral axis of the Drosophila embryo. Development 113, 35-54.

Reichert, H. and Simeone, A. (1999). Conserved usage of gap and homeotic genes in patterning the CNS. Curr Opin Neurobiol 9, 589-95.

Rivera-Pomar, R. and Jäckle, H. (1996). From gradients to stripes in Drosophila embryogenesis: filling in the gaps. Trends Genet 12, 478-83.

Rivera-Pomar, R., Niessing, D., Schmidt-Ott, U., Gehring, W. and Jäckle, H. (1996). RNA binding and translational suppression by bicoid. Nature 379, 746-9.

Rohr, K., Tautz, D. and Sander, K. (1999). Segmentation gene expression in the mothmidge Clogmia albipunctata (Diptera, psychodidae) and other primitive dipterans. Dev Genes Evol 209, 145-54.

Rosenberg, U., Schröder, C., Preiss, A., Kienlin, A., Côté, S., Riede, I. and Jäckle, H. (1986). Structural homology of the product of the Drosophila Krüppel gene with Xenopus transcription factor IIIA. Nature 319, 336-9.

Roth, S., Stein, D. and Nüsslein-Volhard, C. (1989). A gradient of nuclear localization of the dorsal protein determines dorsoventral pattern in the Drosophila embryo. Cell 59, 1189-202.

Rubin, G. and Spradling, A. (1982). Genetic transformation of Drosophila with transposable element vectors. Science 218, 348-53.

Rushlow, C., Colosimo, P., Lin, M., Xu, M. and Kirov, N. (2001). Transcriptional regulation of the Drosophila gene zen by competing Smad and Brinker inputs. Genes Dev 15, 340-51.

Rushlow, C., Doyle, H., Hoey, T. and Levine, M. (1987). Molecular characterization of the zerknüllt region of the Antennapedia gene complex in Drosophila. Genes Dev 1, 1268-79.

Rushlow, C., Han, K., Manley, J. and Levine, M. (1989). The graded distribution of the dorsal morphogen is initiated by selective nuclear transport in Drosophila. Cell 59, 1165-77.

Rushlow, C. and Levine, M. (1990). Role of the zerknüllt gene in dorsal-ventral pattern formation in Drosophila. Adv Genet 27, 277-307.

Sambrook, J. and Russel, D. (2001). Molecular Cloning: A Laboratory Manual. Cold Spring Harbor: Laboratory Press.

Sander, K. (1976). Specification of the basic body pattern in insect embryogenesis. Adv Insect Physiol 12, 125238.

Schmidt, O., Zissler, D., Sander, K. and Kalthoff, K. (1975). Switch in pattern formation after puncturing the anterior pole of Smittia eggs (Chironomidae, Diptera). Dev Biol 46, 216-21. 
Schmidt-Ott, U. (2000). The amnioserosa is an apomorphic character of cyclorrhaphan flies. Dev Genes Evol 210, 373-6.

Schmidt-Ott, U. (2005). Insect serosa: a head line in comparative developmental genetics. Curr Biol 15, R245-7.

Schmidt-Ott, U., Sander, K. and Technau, K. (1994). Expression of engrailed in embryos of a beetle and five dipteran species with special reference to the terminal regions. Roux's Arch Dev Biol 203, 298-303.

Schröder, C., Tautz, D., Seifert, E. and Jäckle, H. (1988). Differential regulation of the two transcripts from the Drosophila gap segmentation gene hunchback. EMBO J 7, 2881-7.

Schröder, R. (2003). The genes orthodenticle and hunchback substitute for bicoid in the beetle Tribolium. Nature 422, 621-5.

Schröder, R. and Sander, K. (1993). A comparison of transplantable bicoid activity and partial bicoid homeobox sequences in several Drosophila and blowfly species (Calliphoridae). Roux's Arch Dev Biol 203, 3443.

Schwalm, F. (1987). Insect Morphogenesis. Basel: S. Karger AG.

Schüpbach, T. and Wieschaus, E. (1986). Maternal-effect mutations altering the anterior-posterior pattern of the Drosophila embryo. Roux's Arch Dev Biol 195, 302-17.

Schüpbach, T. and Wieschaus, E. (1989). Female sterile mutations on the second chromosome of Drosophila melanogaster. I. Maternal effect mutations. Genetics 121, 101-17.

Seeger, M. and Kaufman, T. (1990). Molecular analysis of the bicoid gene from Drosophila pseudoobscura: identification of conserved domains within coding and noncoding regions of the bicoid mRNA. EMBO J 9, 2977-87.

Shaw, P., Salameh, A., McGregor, A., Bala, S. and Dover, G. (2001). Divergent structure and function of the bicoid gene in Muscoidea fly species. Evol Dev 3, 251-62.

Shaw, P., Wratten, N., McGregor, A. and Dover, G. (2002). Coevolution in bicoid-dependent promoters and the inception of regulatory incompatibilities among species of higher Diptera. Evol Dev 4, 265-77.

Shinmyo, Y., Mito, T., Matsushita, T., Sarashina, I., Miyawaki, K., Ohuchi, H. and Noji, S. (2005). caudal is required for gnathal and thoracic patterning and for posterior elongation in the intermediate-germband cricket Gryllus bimaculatus. Mech Dev 122, 231-9.

Simpson-Brose, M., Treisman, J. and Desplan, C. (1994). Synergy between the hunchback and bicoid morphogens is required for anterior patterning in Drosophila. Cell 78, 855-65.

Smith, K. (1969). Diptera: Lonchopteridae. London: Royal Entomological Society.

Sommer, R., Retzlaff, M., Goerlich, K., Sander, K. and Tautz, D. (1992). Evolutionary conservation pattern of zinc-finger domains of Drosophila segmentation genes. Proc Natl Acad Sci U S A 89, 10782-6.

Sommer, R. and Tautz, D. (1991). Segmentation gene expression in the housefly Musca domestica. Development 113, 419-30.

Sonoda, J. and Wharton, R. (1999). Recruitment of Nanos to hunchback mRNA by Pumilio. Genes Dev 13, 2704-12.

Spencer, F., Hoffmann, F. and Gelbart, W. (1982). Decapentaplegic: a gene complex affecting morphogenesis in Drosophila melanogaster. Cell 28, 451-61.

Sprenger, F., Stevens, L. and Nüsslein-Volhard, C. (1989). The Drosophila gene torso encodes a putative receptor tyrosine kinase. Nature 338, 478-83.

Sprenger, F., Trosclair, M. and Morrison, D. (1993). Biochemical analysis of torso and D-raf during Drosophila embryogenesis: implications for terminal signal transduction. Mol Cell Biol 13, 1163-72.

St Johnston, D., Beuchle, D. and Nüsslein-Volhard, C. (1991). Staufen, a gene required to localize maternal RNAs in the Drosophila egg. Cell 66, 51-63. 
St Johnston, D., Driever, W., Berleth, T., Richstein, S. and Nüsslein-Volhard, C. (1989). Multiple steps in the localization of bicoid RNA to the anterior pole of the Drosophila oocyte. Development 107 Suppl, 13-9.

St Johnston, D. and Nüsslein-Volhard, C. (1992). The origin of pattern and polarity in the Drosophila embryo. Cell 68, 201-19.

Stathopoulos, A. and Levine, M. (2002). Dorsal gradient networks in the Drosophila embryo. Dev Biol 246, 5767.

Stauber, M., Jäckle, H. and Schmidt-Ott, U. (1999). The anterior determinant bicoid of Drosophila is a derived Hox class 3 gene. Proc Natl Acad Sci U S A 96, 3786-9.

Stauber, M., Prell, A. and Schmidt-Ott, U. (2002). A single Hox3 gene with composite bicoid and zerknüllt expression characteristics in non-Cyclorrhaphan flies. Proc Natl Acad Sci U S A 99, 274-9.

Stauber, M., Taubert, H. and Schmidt-Ott, U. (2000). Function of bicoid and hunchback homologs in the basal cyclorrhaphan fly Megaselia (Phoridae). Proc Natl Acad Sci U S A 97, 10844-9.

Steward, R. (1987). Dorsal, an embryonic polarity gene in Drosophila, is homologous to the vertebrate protooncogene, c-rel. Science 238, 692-4.

Steward, R. (1989). Relocalization of the dorsal protein from the cytoplasm to the nucleus correlates with its function. Cell 59, 1179-88.

Steward, R., McNally, F. and Schedl, P. (1984). Isolation of the dorsal locus of Drosophila. Nature 311, 262-5.

Strimmer, K. and von Haeseler, A. (1996). Quartet Puzzling: A Quartet Maximum-Likelihood Method for Reconstructing Tree Topologies. Mol Biol Evol 13, 964-9.

Struhl, G. (1989). Differing strategies for organizing anterior and posterior body pattern in Drosophila embryos. Nature 338, 741-4.

Struhl, G., Struhl, K. and Macdonald, P. (1989). The gradient morphogen bicoid is a concentration-dependent transcriptional activator. Cell 57, 1259-73.

Tautz, D. (1988). Regulation of the Drosophila segmentation gene hunchback by two maternal morphogenetic centres. Nature 332, 281-4.

Tautz, D., Lehmann, R., Schnürch, H., Schuh, R., Seifert, E., Kienlin, A., Jones, K. and Jäckle, H. (1987). Finger protein of novel structure encoded by hunchback, a second member of the gap class of Drosophila segmentation genes. Nature 327, 383-9.

Tautz, D. and Pfeifle, C. (1989). A non-radioactive in situ hybridization method for the localization of specific RNAs in Drosophila embryos reveals translational control of the segmentation gene hunchback. Chromosoma 98, 81-5.

Thisse, C., Perrin-Schmitt, F., Stoetzel, C. and Thisse, B. (1991). Sequence-specific transactivation of the Drosophila twist gene by the dorsal gene product. Cell 65, 1191-201.

Thummel, C. and Pirrotta, V. (1992). New pCaSpeR P element vectors. Dros Inf Serv 71, 150.

Treier, M., Pfeifle, C. and Tautz, D. (1989). Comparison of the gap segmentation gene hunchback between Drosophila melanogaster and Drosophila virilis reveals novel modes of evolutionary change. EMBO J 8, 1517-25.

Treisman, J. and Desplan, C. (1989). The products of the Drosophila gap genes hunchback and Krüppel bind to the hunchback promoters. Nature 341, 335-7.

Treisman, J., Gönczy, P., Vashishtha, M., Harris, E. and Desplan, C. (1989). A single amino acid can determine the DNA binding specificity of homeodomain proteins. Cell 59, 553-62.

Vogt, R., Prestwich, G. and Lerner, M. (1991). Odorant-binding-protein subfamilies associate with distinct classes of olfactory receptor neurons in insects. J Neurobiol 22, 74-84. 
Wakimoto, B., Turner, F. and Kaufman, T. (1984). Defects in embryogenesis in mutants associated with the antennapedia gene complex of Drosophila melanogaster. Dev Biol 102, 147-72.

Wang, C. and Lehmann, R. (1991). Nanos is the localized posterior determinant in Drosophila. Cell 66, 63747.

Wharton, R. and Struhl, G. (1991). RNA regulatory elements mediate control of Drosophila body pattern by the posterior morphogen nanos. Cell 67, 955-67.

Wieschaus, E., Nüsslein-Volhard, C. and Jürgens, G. (1984). Mutations affecting the pattern of the larval cuticle in Drosophila melanogaster: III. Zygotic loci on the X-chromosome and fourth chromosome. Roux's Arch Dev Biol 193, 296-307.

Wimmer, E., Carleton, A., Harjes, P., Turner, T. and Desplan, C. (2000). Bicoid-independent formation of thoracic segments in Drosophila. Science 287, 2476-9.

Wolff, C., Schröder, R., Schulz, C., Tautz, D. and Klingler, M. (1998). Regulation of the Tribolium homologues of caudal and hunchback in Drosophila: evidence for maternal gradient systems in a short germ embryo. Development 125, 3645-54.

Wolff, C., Sommer, R., Schröder, R., Glaser, G. and Tautz, D. (1995). Conserved and divergent expression aspects of the Drosophila segmentation gene hunchback in the short germ band embryo of the flour beetle Tribolium. Development 121, 4227-36.

Yajima, H. (1964). Studies on embryonic determination of the harlequin-fly, Chironomus dorsalis. II. Effects of partial irradiation of the egg by ultra-violett light. J Embryol Exp Morphol 12, 89-100.

Yeates, D. and Wiegmann, B. (1999). Congruence and controversy: toward a higher-level phylogeny of Diptera. Annu Rev Entomol 44, 397-428.

Zdobnov, E., von Mering, C., Letunic, I., Torrents, D., Suyama, M., Copley, R., Christophides, G., Thomasova, D., Holt, R., Subramanian, G. et al. (2002). Comparative genome and proteome analysis of Anopheles gambiae and Drosophila melanogaster. Science 298, 149-59.

Zhao, C., Dave, V., Yang, F., Scarborough, T. and Ma, J. (2000). Target selectivity of bicoid is dependent on nonconsensus site recognition and protein-protein interaction. Mol Cell Biol 20, 8112-23. 


\section{CV}

Steffen Joachim Lemke

1983-1987 Grundschule Am Engelnberg, Wuppertal-Elberfeld

1988-1996 Gymnasium Am Kothen, Wuppertal-Barmen

1996-1997 Zivildienst

1997-2000 Undergraduate studies at the Georg August Universität Göttingen

1999 Vordiplom in Molecular Genetics, Plant Physiology, Organic Chemistry and Physical Chemistry

2000-2006 Graduate studies in the MSc/PhD Molecular Biology Program at the Georg August Universität Göttingen.

MSc with Urs Schmidt-Ott: Department of Developmental Biology (Herbert Jäckle) at the Max-Planck-Institut für biophysikalische Chemie, Göttingen, Germany. Topic: Initial Analysis of the Evolution of Bicoid-dependent hunchback Regulation.

2001-2006 PhD with Urs Schmidt-Ott: Department of Developmental Biology (Herbert Jäckle) at the MaxPlanck-Institut für biophysikalische Chemie, Göttingen, Germany. Since 2003: Department of Organismal Biology and Anatomy, The University of Chicago, Chicago, IL, USA. Topic: Evolution of the Bicoid-dependent hunchback regulation in Diptera.

Meeting Presentations

Botanikertagung, 2000, Jena, Germany. Lemke, S., Reumann, S. "Identification of Novel Proteins from Plant Peroxisomes by Bioinformatic Analyses"

44th Annual Drosophila Research Conference, 2003, Chicago, IL. Lemke, S. J., Prell, A. H., Stauber, M., Schmidt-Ott, U. "Evolution of transcriptional control of the Drosophila gap gene hunchback"

45th Annual Drosophila Research Conference, 2004, Washington, DC. Lemke, S. J., Rafiqi, A. M., Prell, A. H., Stauber, M., Schmidt-Ott, U. "Evolution of transcriptional control of the Drosophila gap gene hunchback"

Publications

Reumann, S., Ma, C., Lemke, S., Babujee, L. (2004). AraPerox. A database of putative Arabidopsis proteins from plant peroxisomes. Plant Physiol. 2004 136: 2587-608.

Walther, K., Krauss, M., Diril, M. K., Lemke, S., Ricotta, D., Honing, S., Kaiser, S., Haucke, V. (2001). Human stoned B interacts with AP-2 and synaptotagmin and facilitates clathrin-coated vesicle uncoating. EMBO Rep. 2: 634-640. 